\title{
Culturally-Relevant Persuasive Technology
}

by

Rilla Khaled

\author{
A thesis \\ submitted to the Victoria University of Wellington \\ in fulfilment of the requirements for the degree of \\ Doctor of Philosophy \\ in Computer Science.
}

Victoria University of Wellington 2008 


\begin{abstract}
Persuasive technology (PT) has been defined by B. J. Fogg as "any interactive computing system designed to change people's attitudes or behaviors". The kinds of attitudes people hold, and the behaviours they exhibit, are influenced by culture, so cultural beliefs play a large role in persuasion. The cross-cultural psychology literature has demonstrated the power of culture on attitude change. To date, however, there has been limited persuasive technology research outside of countries characterised as having individualist culture, where individualism describes cultures in which society members have a primarily individual identity. Little research has explicitly investigated the relationship between persuasive technology and culture. In this thesis, we investigated the hypothesis that persuasive technology is more effective when it reflects the culture of its intended target audience. Firstly, we established a set of effective, culturally-relevant persuasive technology strategies, almost all of which are targeted at use in tools for collectivist audiences. The strategies are based on a combination of findings from the cross-cultural psychology literature focused on the dimensions of individualism and collectivism, an analysis of existing PT strategies, and qualitative insights. Secondly, we developed a culturally-relevant PT prototype based on several of our strategies, in the form of two versions of a game titled Smoke? about smoking cessation. One version of Smoke? was designed to be more persuasive for a NZ European player audience, while the other was designed to be more persuasive for a Māori player audience. We conducted focus groups with potential players, the insights of which guided how we applied several of our culturally-relevant PT strategies in the design of the two game versions. Finally, we evaluated both game versions on individualist, and collectivist test players, using both quantitative and qualitative methods. The results of our evaluation support our hypothesis. Not only did both sets of players engage with, and use the culturally-relevant interface elements of the game designed for their culture, they also demonstrated that the culturally-relevant game versions had increased their anti-smoking beliefs.
\end{abstract}




\section{Acknowledgments}

I would like to express my sincere gratitude to a number of people, without the help of whom this thesis would simply not exist.

My three supervisors James Noble, Robert Biddle, and Ron Fischer, all provided me with excellent guidance and support over the course of this study. Their enthusiasm for the topic was invaluable at times when my own enthusiasm was waning.

Everard Halbert and Mena Aukuso were superb gatekeeper ambassadors for my research, and made it possible for me to obtain a Māori and Pacific test player sample. Shona de Sain, Liz Richardson, and Debbie Whare were also very helpful in obtaining players.

Over the course of my study, my family and friends have been very supportive and encouraging, and unexpectedly understanding of my reclusive behaviour during the write-up stage.

Mary Barr, Pippin Barr, my father Mohammed Khaled, and Bella Cawthorn did a fantastic job of wading through these foreign pages, by carefully proofreading and editing chapters. Matt Duignan and Richard Procter kindly provided their excellent expert opinion on Smoke? during the development phase. Pippin, my lovely husband, also provided much ${ }^{1}$ general mental health relief, and took on the role of the world's most patient research assistant.

The participants involved in this study (all 200 of them... give or take a few!) contributed invaluably towards my research, and made the whole experience incredibly rewarding. The social marketing organisation members I interviewed deserve a special mention, as they not only agreed to participate, but maintained ongoing communication with me over the entire duration of my study, which enriched my research.

Finally, without the support of TEC, the Health Sponsorship Council, and the Royal Society of New Zealand, I really would have been a starving student.

\footnotetext{
${ }^{1} \mathrm{SO}$ MUCH!
} 


\section{Contents}

1 Introduction $\quad 1$

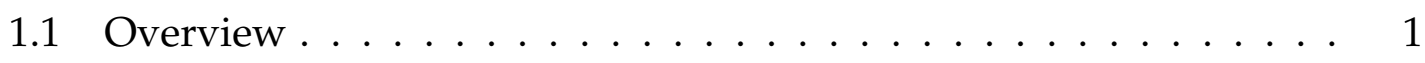

1.2 Problem context . . . . . . . . . . . . . . . . . 1

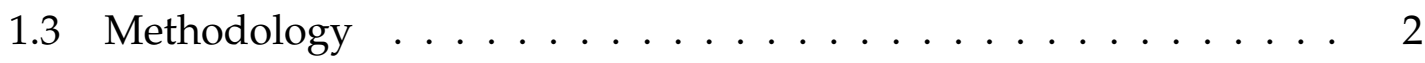

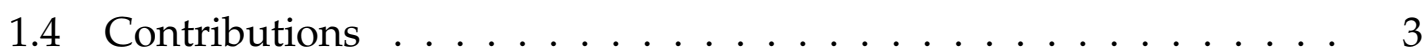

1.5 Delimitations and limitations of the study . . . . . . . . . . . 4

1.6 Thesis structure outline . . . . . . . . . . . . . . . 5

1.7 Publications .................. 7

2 Background 9

2.1 Persuasive technology . . . . . . . . . . . . . . 9

2.1.1 Fogg's PT tool strategies . . . . . . . . . . . . . 10

2.1.2 Persuasive technology research . . . . . . . . . . . . 11

2.1.3 Games as persuasive technology . . . . . . . . . . . . . . 14

2.1 .4 Discussion . . . . . . . . . . . . . . 17

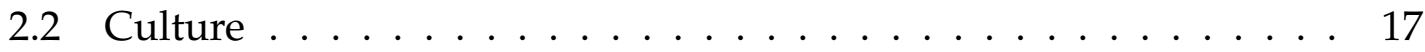

2.2 .1 Definitions . . . . . . . . . . . . . 17

2.2.2 Cross-cultural comparisons . . . . . . . . . . . 18

2.2 .3 Discussion ...................... 21

2.3 Cultural aspects of HCI . . . . . . . . . . . . . . . 23

2.3.1 Covert culture . . . . . . . . . . . . . . 23

2.3.2 Cultural metaphors . . . . . . . . . . . . . 24

2.3.3 Cultural dimensions . . . . . . . . . . . . . . . . . 24

2.3 .4 Discussion . . . . . . . . . . . . . . 25

2.4 Cross-cultural psychology and consumer behaviour . . . . . . . 26

2.4.1 Cultural styles in advertising . . . . . . . . . . . 27

2.4.2 Individualism, collectivism, and persuasion . . . . . . . 27

2.4 .3 Stability of cultural preferences . . . . . . . . . . . 28

2.5 Limitations of the current approaches . . . . . . . . . . . . . . 29 
3 Methodology 31

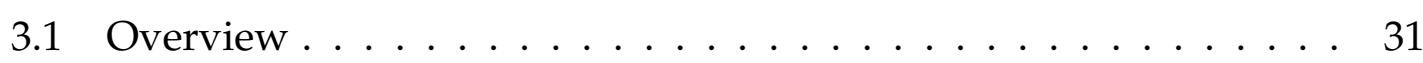

3.1.1 Culture and persuasion .............. 31

3.1 .2 Research design . . . . . . . . . . . . . . . 33

3.1.3 Research phases.................. 34

3.1.4 Researcher bias ................. . . 34

3.2 Theory Development . . . . . . . . . . . . . . . 35

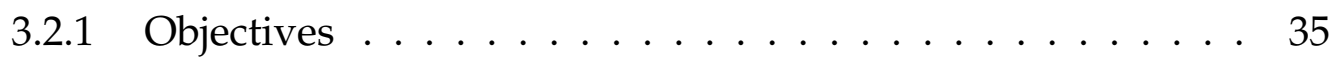

3.2 .2 Process and methods . . . . . . . . . . . . . 36

3.3 Prototype Development . . . . . . . . . . . . . . . 38

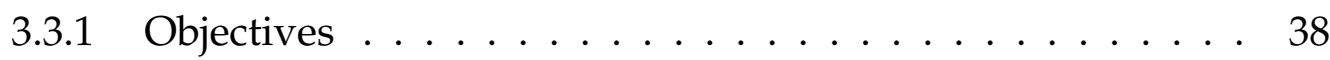

3.3.2 Process and methods . . . . . . . . . . . . . . 41

3.4 Evaluation . . . . . . . . . . . . . . . . 45

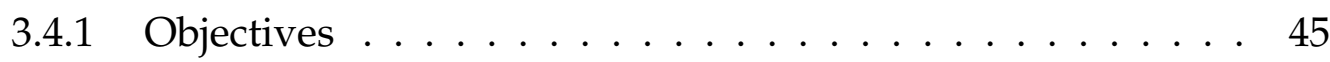

3.4 .2 Process and methods . . . . . . . . . . . . . . 46

3.5 Delimitations, limitations, and other study considerations . . . . . 49

4 Culturally-Relevant PT Strategies 53

4.1 Social marketing stakeholder interviews . . . . . . . . . . . . . 53

4.1.1 Universal relevance: the views of NZ European stakeholders 54

4.1.2 Reduction of relevance: the views of Māori stakeholders . . 55

4.1.3 A collective focus: the views of Māori stakeholders . . . . . 56

4.2 Fogg's PT strategies from a context of individualism and collectivism 58

4.2.1 The REDUCTION and TUNNELLING strategies . . . . . . . . 59

4.2 .2 The TAILORING strategy . . . . . . . . . . . . 60

4.2 .3 The SUGGESTION strategy . . . . . . . . . . . . . 61

4.2.4 The SELF MONITORING strategy . . . . . . . . . . . 62

4.2 .5 The SURVEILLANCE strategy . . . . . . . . . . . 62

4.2.6 The CONDITIONING strategy . . . . . . . . . . . . 64

4.3 A set of eight culturally-relevant PT design strategies . . . . . . . 65

4.3.1 The GROUP CUSTOMISATION strategy . . . . . . . . . . . . 66

4.3 .2 The HARMONY strategy . . . . . . . . . . . . . 68

4.3.3 The GROUP OPINION strategy . . . . . . . . . . . . . . 69

4.3.4 The MENTORING strategy . . . . . . . . . . . . 71

4.3.5 The DIFFERENCE MONITORING strategy . . . . . . . . . 73

4.3.6 The DISESTABLISHING strategy . . . . . . . . . . 75

4.3.7 The TEAM PERFORMANCE strategy . . . . . . . . . . . 77

4.3.8 The ACHIEVEMENT strategy . . . . . . . . . . . 78 
5 Smoke? Game mechanics $\quad 83$

5.1 Initial design decisions . . . . . . . . . . . . . . 83

5.1 .1 The starting point . . . . . . . . . . . . . 83

5.1 .2 Game objectives. . . . . . . . . . . . . . . 84

5.1 .3 Determining game genre . . . . . . . . . . . 85

5.1.4 Conceptual overview of Smoke? . . . . . . . . . . . . . . . 87

5.2 Concrete design decisions . . . . . . . . . . . . . . . . 90

5.2.1 Game narrative . . . . . . . . . . . . . . . . . 90

5.2.2 Game design concepts ................ 92

6 Smoke? Incorporating culture $\quad 103$

6.1 Focus group insights . . . . . . . . . . . . . . . . 103

6.1.1 Willingness to engage with social marketing . . . . . . . 104

6.1 .2 Taking up smoking . . . . . . . . . . . . . . . . . . . . . . . . . . . . . . . . . . . .

6.1 .3 Smoking as a habit . . . . . . . . . . . . 105

6.1 .4 Locus of smoking . . . . . . . . . . . . . 106

6.1.5 Reasons for wanting to quit smoking . . . . . . . . . 108

6.1.6 Opinions on characteristics of effective appeals . . . . . . . 109

6.2 Incorporating insights into the design . . . . . . . . . . . . . . 111

6.2.1 Insights into design: common features . . . . . . . . . . . . 111

6.2.2 Insights into design: the NZE version . . . . . . . . . . . 113

6.2.3 Insights into design: the NZM version . . . . . . . . . . . . 115

6.3 Incorporating the strategies into Smoke? . . . . . . . . . . . . . . . . 121

6.3.1 Decision and incorporation process . . . . . . . . . . . 121

6.3.2 Tactics into design: the NZE version . . . . . . . . . . . . . 122

6.3.3 Tactics into design: the NZM version . . . . . . . . . . . 127

7 Smoke? Play testing and refinement $\quad 135$

7.1 Play testing details . . . . . . . . . . . . . . 136

7.1.1 User evaluations . . . . . . . . . . . . . 136

7.1.2 Expert evaluations . . . . . . . . . . . . 137

7.2 User evaluation themes . . . . . . . . . . . . . . . . . . 139

7.2.1 Managing player attention . . . . . . . . . . . 139

7.2.2 Balancing replay with reality . . . . . . . . . . . . . . . 142

7.2.3 Player control vs. system control . . . . . . . . . . . . . 144

7.2 .4 Identity issues . . . . . . . . . . . . . . . . 146

7.3 Expert evaluation themes . . . . . . . . . . . . . 147

7.3.1 Adopting "traditional" interface norms . . . . . . . . . . . 147

7.3.2 Feedback about changes to game state . . . . . . . . . . 149 
7.3.3 Reduction of text instructions . . . . . . . . . . . . . . 150

7.3.4 Fixing game world inconsistencies . . . . . . . . . . . 151

8 Evaluation $\quad 153$

8.1 The quantitative evaluation . . . . . . . . . . . . . . 153

8.1.1 Method ................... 153

8.1 .2 Analyses and results . . . . . . . . . . . . . 158

8.1 .3 Discussion . . . . . . . . . . . . . . 166

8.2 The qualitative evaluation $\ldots \ldots \ldots \ldots$. . . . . . . . . . . . . . . . .

8.2.1 Method ..................... 166

8.2.2 Findings for the NZE version . . . . . . . . . . . . . 167

8.2.3 Findings for the NZM version . . . . . . . . . . . 177

8.3 Discussion . . . . . . . . . . . . . . . . 186

8.3.1 Support for our hypothesis . . . . . . . . . . . 186

8.3.2 Assessment of the design strategies . . . . . . . . . 187

9 Conclusions $\quad 189$

9.1 Contributions . . . . . . . . . . . . . . . . . . . . . 191

9.1.1 An original set of culturally-relevant PT design strategies . 191

9.1.2 A prototype of a culturally-relevant persuasive technology . 191

9.1.3 Evaluation of the culturally-relevant persuasive technology 192

9.2 Delimitations and limitations of the study . . . . . . . . . . . . . . 193

9.3 Related work . . . . . . . . . . . . . . . . . . . . . . . . 194

9.3.1 PT research . . . . . . . . . . . . . . . . . 194

9.3 .2 Cultural aspects of HCI . . . . . . . . . . . . 195

9.3.3 Consumer psychology focused on individualism and collectivism . . . . . . . . . . . . . . . . 195

9.4 Future work . . . . . . . . . . . . . . . . . 195

9.5 Summary . . . . . . . . . . . . . . . . 197

$\begin{array}{ll}\text { A Case Study Information } & 199\end{array}$

B Case Study Consent Form $\quad 203$

C Case Study Interview Outline 205

D Focus Group Information $\quad 207$

E Focus Group Consent Form $\quad 211$

F Focus Group Agenda $\quad 213$ 
G Game Tester Information

H Game Tester Consent Form

I Smoke survey 


\section{List of Figures}

2.1 Schwartz's values continuum with cultural dimensions . . . . . . . 22

4.1 A motivation-based arrangement of the PT strategies . . . . . . . 81

5.1 An early outline of messages to highlight to players . . . . . . . . 86

5.2 Early planning for the game . . . . . . . . . . . . 88

$5.3 \mathrm{MC}$ and her mother in the dining room . . . . . . . . . . . . 89

5.4 A skeleton plan for week 1, day $1 \ldots$. . . . . . . . . . . . 91

5.5 Early draft of dialogue from week 1 day 1 of the NZE version of Smoke? . . . . . . . . . . . . . . . . . 91

5.6 An early plan for the phrase component . . . . . . . . . . . . . . . 93

5.7 A high-level diagram of the narrative of Smoke? . . . . . . . . . . . 94

5.8 The NZE version of the status screen . . . . . . . . . . . . . . . 95

5.9 The countdown box . . . . . . . . . . . . . . . . . . . 96

5.10 Option to add "call Jake" as a craving control activity . . . . . . . . . 97

5.11 Options of craving control activities when social support is a craving context . . . . . . . . . . . . . . . . 97

5.12 Wildcard scene outcome . . . . . . . . . . . . . . . . . . . . 98

5.13 A timeline for the narrative of Smoke? . . . . . . . . . . . . . . . . . 99

5.14 Update of stagnant time between weeks 1 and 3 . . . . . . . . 100

5.15 A one year outcome for the game . . . . . . . . . . . . . . 101

6.1 A neighbourhood background in the Flash development tool . . . . 112

6.2 A chance encounter between MC and a family friend . . . . . . . . 114

6.3 The fitness-running minigame . . . . . . . . . . . . . . . 115

6.4 References to future plans in the outcome sequence . . . . . . . . 116

6.5 Emphasising monetary savings . . . . . . . . . . . . . . . . . 116

6.6 Jade asks MC a sensitive question . . . . . . . . . . . . . . . 118

$6.7 \mathrm{MC}$ 's best friend explaining her recent absence . . . . . . . . . . . . 119

6.8 The self-esteem score from the status screen . . . . . . . . . . . . . . 123

6.9 The concentration-shopping minigame . . . . . . . . . . . . . . . 124 
6.10 The shopping with savings option . . . . . . . . . . . . 125

6.11 Shopping for an iPod . . . . . . . . . . . . . . . . 126

6.12 The moods + opinions box . . . . . . . . . . . . . . . . . . . 127

6.13 Gran's opinion of MC taking Arihia's phone number . . . . . . . . 128

6.14 Gran in hospital talking to MC and Jade . . . . . . . . . . . . . 129

$6.15 \mathrm{MC}^{\prime}$ s best friend offers MC a smoke . . . . . . . . . . . . . . . . . . 129

6.16 The concentration-sandwich minigame . . . . . . . . . . . . 131

6.17 The respect score from $\mathrm{MC}^{\prime}$ s status screen . . . . . . . . . . . . . . 132

6.18 Convincing the best friend to quit . . . . . . . . . . . . . . . 133

6.19 Auntie directing her thoughts on smoking at the best friend . . . . 134

7.1 Notes from the design journal on change suggestions . . . . . . . 136

7.2 The initial version of the status screen . . . . . . . . . . . . . 140

7.3 The final version of the status screen . . . . . . . . . . . . . . 141

7.4 replay functionality representations in the game . . . . . . . . . . . . 143

7.5 The initial version of the wildcard scene interface . . . . . . . . . . 144

7.6 The final version of the wildcard scene interface . . . . . . . . . . . 146

7.7 Off, over, and press states of the conversation window forward button . . . . . . . . . . . . . . . . . . . . . . 148

7.8 Pointing at a component vs. grabbing a component . . . . . . . . . 149

7.9 A mood + opinion face before and after a mood change . . . . . . . 150

7.10 Three interaction mode icons . . . . . . . . . . . . . . . . . . . . . . . 151

8.1 A bar graph of the paired means of five attitude groups . . . . . . 159

8.2 Changes in subjects' post- and pre- intention to quit by cultural orientation and game version . . . . . . . . . . . . . . . 161

8.3 Changes in subjects' post- and pre- resistance to smoking by cultural orientation and game version . . . . . . . . . . . . . 162

8.4 Changes in subjects' pre- and post- temptation to smoke by cultural orientation and game version . . . . . . . . . . . . . 165 


\section{Chapter 1}

\section{Introduction}

\subsection{Overview}

Persuasive technology (PT) has been defined by B. J. Fogg as "any interactive computing system designed to change people's attitudes or behaviors" [46]. The kinds of attitudes people hold, and the behaviours they exhibit, are influenced by culture [62, 69, 142], so cultural beliefs play a large role in persuasion [38]. The cross-cultural psychology literature has demonstrated the power of culture on attitude change, showing that the cultural beliefs of audiences have a significant impact on how they perceive and receive persuasive messages $[1,61,63]$. The literature also shows us that certain tactics and strategies will work effectively with an audience of a particular culture, while the same strategies may be ineffective on an audience of a different culture [23,91,139]. In recent years, the amount of research interest in persuasive technology has increased greatly. To date, however, there has been limited persuasive technology research outside of countries characterised as having individualist culture, where individualism describes cultures in which society members have a primarily individual identity. More specifically, little research has investigated the relationship between persuasive technology and culture. In this thesis, we investigate the following hypothesis:

Persuasive technology is more effective when it reflects the culture of its intended target audience.

\subsection{Problem context}

The potential application area for persuasive technology is vast, including politics, religion, gaming, self-improvement, occupational effectiveness, empathetic skills, marketing, health, safety, and environmental conservation [46, 82]. Persuasive technology is currently being developed in a multitude of forms which in- 
clude portable hand-held devices [56], web-based applications [132], stand-alone programs [44], robots [37], and computerised toys [46], to name a few.

Falling under the umbrella of persuasive technology are games designed to change people's attitudes or behaviours, developed with the objective of imparting knowledge of real world utility to players. These include advergames, "the use of interactive gaming technology to deliver embedded advertising messages to consumers" [32], and persuasive games, defined by Ian Bogost as "videogames that mount procedural rhetorics effectively" [24]. They may also include serious games, defined by David Whatley as any game that is "not specifically entertainment, but which uses entertainment or the techniques and processes of the entertainment business, to achieve a purpose" [154]. One commonly cited example of a serious game is America's Army [146], which was developed in collaboration with the United States Army. It reflects army culture and values, and functions as a recruitment tool [90].

Fundamentally, all forms of persuasive technology exist to persuade. Despite the close links between persuasion and culture, the available literature has not examined the role of culture. While culture has been acknowledged as an important factor in Human-Computer Interaction (HCI) research [40, 148, 149, 159], the literature on culture and HCI has focused on applications and contexts in which persuasion is not a design objective, and therefore does not address how to deal with culture in designing persuasive technology.

Very little research has focused on technology at the intersection of persuasive technology, HCI, and cross-cultural psychology, so this type of technology has remained unnamed. We refer to it as culturally-relevant persuasive technology, which we define as:

Interactive computing products created for the purpose of changing people's attitudes or behaviours, that reflect the cultural motivations of their target users.

\subsection{Methodology}

To investigate our hypothesis, we structured our study in three phases.

Theory development The first phase focused on establishing a theoretical basis for culturally-relevant persuasive technology. We conducted interviews with stakeholders of New Zealand organisations involved with social marketing, defined as "the use of marketing principles and techniques to influence a target 
audience to voluntarily accept, reject, modify or abandon a behavior for the benefit of individuals, groups, or society as a whole" [87]. Our interviews served to investigate whether culture-specific persuasive approaches played a part in the organisations' campaigns. We also examined the motivations underlying the PT design strategies listed in Fogg's book Persuasive Technology [46], in the context of the cultural dimensions of individualism and collectivism. Fogg described these strategies as the most commonly used techniques in PT tools at the time of publication [46]. Combining the findings from the interviews and the motivation examination with existing cross-cultural psychology theory focused on the motivations of individualists and collectivists, we established a set of culturallyrelevant PT design strategies from the cultural perspectives of individualism and collectivism.

Prototype development The second phase concerned the development of a PT prototype. We developed two culturally-relevant versions of a PT computer game promoting smoking cessation, titled Smoke? and targeted at New Zealand (NZ) players. One version is designed for a NZ European audience, while the other is for a Māori audience ${ }^{1}$. To get some insight into how smoking is perceived by these different audiences, we carried out focus groups with potential players. The feedback from the focus groups informed how we harnessed some of our culturally-relevant PT design strategies in the two versions of the game.

Evaluation The final phase focused on the evaluation of our prototype, and more generally, our theory. We conducted a large-scale quantitative and a smallscale qualitative evaluation of the game versions on their players. We looked at both whether the game was generally effective in increasing people's antismoking attitudes, and also how the effectiveness of the game versions differed across audiences. Specifically, we investigated how effective the New Zealand European (NZE) version of the game was on individualist players, as compared to collectivist players, as well as how effective the Māori (NZM) version of the game was on collectivist players, compared to individualist players.

\subsection{Contributions}

With this research, we make three key contributions to the PT literature:

1. An original set of culturally-relevant PT design strategies grounded in extant cross-cultural psychology literature, intended for use in the design of

\footnotetext{
${ }^{1}$ Māori are the indigenous people of New Zealand.
} 
any PT tool harnessing culture as an important factor (see chapter 4).

2. Two versions of a culturally-relevant persuasive technology designed using our set of culturally-relevant PT strategies, with one version targeted at an individualist, NZ European audience, and the other targeted at a collectivist, Māori audience (see chapters 5, 6, and 7).

3. An evaluation of the two versions of the culturally-relevant persuasive technology using both quantitative and qualitative methods to investigate both their overall effectiveness, and differences in their effectiveness on individualist and collectivist player samples (see chapter 8).

\subsection{Delimitations and limitations of the study}

Perspective on culture and cultural dimensions The theory-driven stages of this work, including the examination of Fogg's strategies and the discussion of culturally-relevant PT strategies, reference the cross-cultural psychology literature on individualism and collectivism.

Domains informing our theory During the theory development phase of this study, we interviewed stakeholders belonging to organisations falling under the umbrella of social marketing. That is, we did not focus on commercial organisations. The organisations we involved serve to promote financial literacy and tobacco control.

Form of our PT We have used games as a platform for our culturally-relevant persuasive technology. This impacted on both how we were able to apply our design strategies, and also the player evaluations of our culturally-relevant persuasive technology.

Domain of our PT In step with our focus on social marketing, we chose smoking cessation as the topic of our culturally-relevant persuasive technology. Smoking is a widespread issue in NZ, affecting both the individualist NZ European population and the collectivist Māori population.

Demographics Variables such as age, gender, and socio-economic background play a powerful role in shaping people's attitudes and preferences. Given the short time-frame for this research, however, we decided to not control these variables. During the development and evaluation phases of this study, we have 
analysed data in light of research participants' self-identified cultural affiliations and values.

Cultural classification Where this research calls for categorisations of people's culture and values, we use a shortened version of Schwartz's values survey (see $\S 2.2 .2 .4$ ). In comparison to more general cultural dimensions research, Schwartz's values survey is both more immediately applicable for categorisation purposes, and is supported by greater empirical validation.

Target audiences of our PT The design strategies we propose in Chapter 4 are contextualised within the cultural dimensions categories of individualism and collectivism. Our PT prototype, however, is contextually-based within New Zealand. One version was designed for individualist NZ European players and the other was designed for collectivist Māori players. Both versions were tested by NZ European, Māori, Pacific, Chinese, and Indian participants. As such, our evaluation of the prototype is necessarily limited in terms of its generalisability.

Time span of evaluation Our PT prototype was designed to be played more than once, as it represents different scenarios as a consequence of adopting different courses of action. Each participant involved in its evaluation, however, played it through just once, and provided responses for it immediately after playing. Their responses therefore reflected attitudes and intentions, as opposed to behavioural changes, and a short-term, single-exposure to the game, as opposed to repeated exposure. We expect that repeated exposure to the game may have yielded stronger effects in the participants.

\subsection{Thesis structure outline}

The body of this thesis is structured as follows.

Chapter 2: Background We survey the current literature in related fields of research, as well as relevant definitions and theory. We commence by discussing some of the theory of persuasive technology, and the PT literature. Next, we present a working definition for the concept of culture, and review models of culture that have been influential in our research. Finally, we overview some of the existing HCI research focusing on culture, and look at a sample of the cross-cultural consumer psychology research. 
Chapter 3: Methodology We argue that culture needs to be taken into account in designing persuasive technology. Next, we propose culturally-relevant persuasive technology, and position our research hypothesis. We then overview the methods and processes involved in the three phases of our study.

Chapter 4: Theory We summarise findings from interviews conducted with NZ social marketing organisation stakeholders, investigating their use of culturallyrelevant persuasive approaches in campaigns. We also examine the motivations underlying Fogg's PT tool strategies, in the context of individualism and collectivism. We then present our culturally-relevant PT design strategies.

Chapter 5: Smoke? Game mechanics We describe the design process adopted in establishing the game mechanics of both versions of our culturally-relevant PT, Smoke? We also discuss the functionality of the key components of Smoke? and how they work together.

Chapter 6: Smoke? Incorporating culture We summarise findings from focus groups we conducted on perceptions of smoking and social marketing, featuring NZ European and Māori participants. We then discuss how the findings informed both the general content of the NZ European and Māori versions of Smoke? as well as our application of some of the culturally-relevant PT strategies in the design of the two game versions.

Chapter 7: Smoke?: Play testing and refinement We present the findings of play tests on an initial prototype of Smoke?, conducted with potential players and expert developers. We also discuss how their evaluation findings informed the design of the final versions of Smoke?

Chapter 8: Evaluation We present two evaluations conducted on the final prototypes of the Smoke? game. We examine the results of a quantitative evaluation on the effectiveness of both versions of Smoke? on NZ European and Māori player samples, and also discuss qualitative insights obtained through user observation, the think aloud protocol, and interviews.

Chapter 9: Conclusion We conclude by presenting the contributions of this research. We then discuss how it relates to other work, and suggest directions in which it could be extended in the future. 


\subsection{Publications}

Part of the research discussed in this thesis has appeared in other publications. Several of these publications were co-authored with others, but their content was primarily the work of the author of this thesis. We describe here which parts of the thesis are based on previously published work.

Chapter 4: Theory We presented an early version of the analysis of B.J.Fogg's PT strategies from a context of individualism and collectivism in Developing Culturally-Aware Persuasive Technology, published in the Proceedings of the 7th International Workshop on Internationalisation of Products and Systems, 2005 [81].

We discussed themes from the interviews we conducted with social marketing organisation stakeholders, and suggested an early set of PT strategies for collectivist audiences in Our place or mine? Exploration into CollectivismFocused Persuasive Technology Design, published in the Proceedings of PERSUASIVE '06: The First International Conference on Persuasive Technology for human well-being, 2006 [78]

From a context of game design, we also presented other PT strategies for collectivists in Persuasive Interaction for Collectivist Cultures, published in the Proceedings of The Seventh Australasian User Interface Conference, 2006 [79].

Chapter 5: Smoke? Game mechanics We overviewed the basic game mechanics for Smoke? in Persuasive Interaction for Collectivist Cultures, published in the Proceedings of The Seventh Australasian User Interface Conference, 2006 [79].

Chapter 6: Smoke? Incorporating culture A summary of the themes of focus groups we conducted with NZ European and Māori participants on the topic of smoking, and an application of how they could inform the design of Smoke? was presented in Factoring Culture into the Design of a Persuasive Game, published in the Proceedings of OzCHI: Australasian Computer-Human Interaction Conference, 2006 [77].

Chapter 7: Smoke? Play testing and refinement We discussed the results of the user evaluations of the initial prototypes of Smoke? in Fine Tuning Reality in Persuasive Games, published in Proceedings of PERSUASIVE '07: The Second International Conference on Persuasive Technology, 2007, [80]. 


\section{Chapter 2}

\section{Background}

The research discussed in this dissertation relies on the linkage of a number of key themes. We therefore present this chapter in sections, each discussing key concepts and existing literature related to the themes of our study. We begin by overviewing the research domain of persuasive technology (\$2.1). We continue by explaining the literature on culture, and reviewing the cultural dimensions models that have informed our research (§2.2). We then survey the HCI literature related to culture and cultural dimensions (§2.3). From there, we look at some findings from the domain of cross-cultural consumer psychology (§2.4), and discuss why the research we have overviewed does not address the issue of designing culturally-relevant persuasive technology (\$2.5).

\subsection{Persuasive technology}

B. J. Fogg defined the term persuasive technology to describe "any interactive computing system designed to change people's attitudes or behaviors" [46]. Central to the concept of persuasive technology is the notion of persuasion, which Fogg explained as "an attempt to shape, reinforce, or change behaviors, feelings, or thoughts about an issue, object, or action" [43].

Persuasive technology is currently being developed in a multitude of forms, such as for portable hand-held devices [56], web-based applications [132], standalone programs [44], robots [37], and computerised toys [46]. The potential application areas for persuasive technology include politics, religion, gaming, selfimprovement, occupational effectiveness, empathetic skills, marketing, health, safety, and environmental conservation [46, 82].

According to Fogg, there are three criteria a technology must meet to be considered a persuasive technology. Firstly, the persuasion needs to be a product of human-computer interaction (HCI), as opposed to computer-mediated com- 
munication. Secondly, the potential persuasive effects must be intentional and planned, as opposed to being by-products of using the technology. Thirdly, the persuasive intent must be endogenous, or "built-in" to the product, such that the persuasive or motivational appeals come from the product itself, as opposed to exogenous, whereby persuasive appeals come from an outside source.

Fogg established three types of roles that persuasive technologies could be grouped under, namely: tools, media, and actors. As tools, persuasive technologies typically simplify or facilitate certain activities. As interactive media, persuasive technologies can allow people to experience information specifically developed to shape their opinions. Finally, as actors, persuasive technologies can take on pseudo-human roles.

\subsubsection{Fogg's PT tool strategies}

In this research, we have focused explicitly on persuasive technology in the role of tools. Here, we briefly summarise Fogg's PT tool strategies. Fogg discussed these strategies as being those that are most typically encountered in persuasive technology tools [46].

Reduction Reduction technologies are used to make a complex task simpler, usually by eliminating some of the steps necessary in the step sequence to achieve a certain goal. For example, "one-click" shopping at www. amazon.com speeds up the process of purchasing books online.

Tunnelling Tunnelling technologies are designed to reduce uncertainty, by prompting users through a set sequence of actions or events, step by step, to encourage certain behaviours (and/or change old ones). For example, typical online software registration steps its users through the process.

Tailoring Tailoring technologies provide users with information relevant to them, with the purpose of encouraging them to change certain attitudes or behaviours. They are effective because people tend to pay more attention to information which they believe is tailored for them. For example, online savings calculators at a financial planning advice website ask users to fill in details about their spending habits, and this is used to tailor feedback about potential saving options.

Suggestion Suggestion technologies are premised on the idea of intervening at the most useful time. For some behaviours, people feel more motivated to do 
them at certain times over others. Suggestion technologies are designed to identify these times in advance and then remind users to perform them at these times. For example, OOS/RSI break reminder software reminds users approximately every 15 minutes to take breaks from typing, by displaying messages, or preventing further typing for a brief period.

Self monitoring Self monitoring technologies facilitate people in undertaking typically tedious monitoring tasks. The monitoring information provided serves to inform them on how they might modify their attitudes or behaviours to achieve a related predetermined goal. For example, pedometers relay information about how many steps have been taken, to provide information about progress towards fitness goals.

Surveillance Surveillance technologies allow one party to monitor the behaviour of another party through observation. When people know they are being watched, they tend to behave differently. For example, speeding drivers often slow down when passing through areas where "hidden" speed cameras are known to be located.

Conditioning Conditioning technologies work by making use of operant conditioning to reinforce target behaviours when they occur. Usually the conditioning takes the form of positive reinforcement, such as rewards for performing the target behaviour. For example, many videogames reward players with an increase in game scores for performing well in the context of the gameplay.

\subsubsection{Persuasive technology research}

In the PT literature, much of the research being conducted concerns topics within the scope of health and well-being, marketing, social awareness, self-efficacy, occupational effectiveness, and empathetic skills. As our own study deals with a health and well-being application, we overview a sample of the health and wellbeing PT literature, as well as other studies that intersect with our research interests.

\subsubsection{Health and well-being}

One health-related persuasive technology that uses the SUGGESTION strategy is the "just in time" dietary improvement tool developed by Stephen Intille et al. [74]. The tool presents dietary improvement information at the point of purchase 
to encourage users to make wiser food choices. Other researchers investigating ways to design nutrition tools include Andrea Grimes and Rebecca Grinter, who discuss a hypothetical persuasive game titled OrderUP! for low-income African American groups [57]. Their research is still in very early stages, but they discuss how cultural relevancy, modelling health behaviour, and encouragement of healthy behaviour through the use of social psychology persuasion theory are important considerations in the design of technology. Grimes and Grinter refer to a body of social marketing literature on cultural relevancy which seems particularly focused on African Americans [7, 156].

Health-related virtual mentors have been a topic of interest for several PT researchers. Anke Eyck et al., for example, were interested in the effectiveness of virtual coaches on exercise [41]. They developed a prototype virtual coach, and showed through experimentation that the virtual coaches motivated people both intrinsically and extrinsically to cycle further than they would have done in the absence of the virtual coach. Also interested in virtual mentors, Jorne Grolleman et al. have focused specifically on virtual smoking cessation advisors [58]. They are developing a prototype embodied conversation agent (ECA). This prototype is based on personal intervention coaches who have been proven to be effective in lowering smoking rates among Dutch smokers.

Another tool that aims to lower smoking rates is a virtual environment game developed by researchers of the Virtual Reality Medical Center [155]. It is an intervention tool which is targeted at adolescents, and makes use of cue exposure therapy to desensitise its users to smoking triggers. It also aims to inform its users about high risk craving situations. The tool was trialled on 15 adolescent test participants, who were surveyed before and after using the virtual environment. Their post-survey responses indicated that they had changed their beliefs about smoking-related habits after use.

Continuing the theme of smoking cessation, and of particular relevance to our study, STOMP: Stop Smoking With Mobile Phones is an ongoing project of the Clinical Trials Research Unit of the University of Auckland in New Zealand [28]. It is a cessation support service delivered over the mobile phone. For the six weeks following users quitting, they are sent five messages a day, which are related to smoking cessation advice and support. Gradually the number of messages sent each day is reduced. Preliminary results of the trial showed that the program improved the rates of success in people's short-term self-reported quit rates, as compared to the control situation with no support program. A key component of the STOMP study was culture. Smoking rates of Māori are significantly higher than those of NZ Europeans, and have also been decreasing at a slower rate [135]. This seems to indicate that current cessation persuasion approaches in New Zealand 
are less effective on Māori than on NZ Europeans. The STOMP researchers designed two sets of mobile phone messages: a general set, which was delivered to NZ European participants, and a Māori set, which was delivered to Māori participants. Along with standard smoking cessation advice, the Māori set of messages made use of Māori language and referenced Māori traditions. A promising finding of this research was that the percentage of successful quitters among the Māori STOMP trial participants was the same as that of the NZ European trial participants. The Māori-tailored STOMP service therefore delivered as much benefit to its Māori users as the general service delivered to its NZ European users. In the study, all participants of the NZ European user group received only the general messages, and all Māori participants received only the Māori-tailored messages. An interesting question that researchers did not investigate is to what extent the message differentiation can be attributed to the equal rates of success amongst the two populations. In other words, whether the two populations would have benefited equally from either set of messages.

\subsubsection{Online credibility and trust}

Online marketing is presently a major application area for persuasive technology. As such, research on online credibility and trust has contributed much to the PT literature. Numerous academics have investigated conditions around online trust. B.J. Fogg et al., for example, conducted a long term study with US and Finnish participants to establish which factors affect people's perceptions of the credibility of a website [45]. The results of these surveys led to the development of design implications that can help developers boost a website's credibility. Three of these implications are: add value to each web visit, guard the integrity of your content, and make it simple to get around.

Carole Bonanni and Diana Cyr are presently carrying out a three year study to investigate the relationship between e-loyalty, website design, and culture [26]. They focus on uncovering to what degree predispositions for online trust differ across cultures (specifically Canada, the US, Germany, and Japan). Questions guiding the current research include whether there are differences across cultures related to payment security, perceived company legitimacy, and assurance. Other research questions include whether local site design elements are preferred to ones perceived as foreign, and whether these local design elements will lead to higher levels of website trust, website perception, and website satisfaction. 


\subsubsection{Ethics and boundaries}

As persuasive technology is concerned with behavioural and attitudinal change, ethics are an ongoing concern. Daniel Berdichevsky and Erik Neuenschwander proposed a framework for analysing acts of persuasion according to their motivations, methods, and outcomes, which distributes responsibility between the designer of a persuasive technology and the user [20]. The "golden rule" guiding their overall framework is the following principle: the creators of a persuasive technology should never seek to persuade anyone of something they themselves would not consent to be persuaded of [20]. Carey Heckman and Jacob Wobbrock explored how anthropomorphic agents that leverage humanistic qualities can be misleading and endanger electronic consumers [66]. The researchers urged designers to remember that people tend to assume that they are dealing with other people, and suggested guidelines for reducing the risks in misleading them in anthropomorphic agent design. Their guidelines include creating humble agents, and avoiding unnecessary realism.

Much of the research just described can be classified as persuasive, but there are cases in which persuasion is intentional but less obvious. From a design philosophy perspective, Johan Redstrom argues for a need to broaden the discourse on persuasive technology [118]. Redstrom views the boundaries of persuasive design as difficult to define, and paraphrases Fogg to state that "technology always changes what we think and do" [118]. In particular, Redstrom focuses on the idea of arguments as material, that is, the notion that the physical form of objects influences us in matters of use or intended use. Redstrom's view relates to James Gibson's and Donald Norman's research on affordance theory [55, 106].

\subsubsection{Games as persuasive technology}

The persuasive technology umbrella also extends to games designed to change people's attitudes or behaviours and developed with the objective of imparting to players knowledge that has effects in the real world. One simple but compelling game is the well-known September 12th [48]. It is a small, web-based Flash game, with limited interactivity, which uses a simulation mechanic to express one view of how civilians become terrorists, and the general spread of terrorism. Of note here is that two populations are depicted: civilians and terrorists, but it would seem that the target audience of the game is people who align themselves with the civilians, that is, the general public. September 12th makes a clear attempt to understand the viewpoints of different populations. Another game illustrating the perspective of a minority culture is Darfur is Dying [122]. A web-based Flash 
simulation game as well, it highlights some of the hardships faced by Darfurian refugees. It was co-developed by MTV, Reebok, and students from the University of Southern California.

\subsubsection{Serious games}

Both September 12th and Darfur is Dying might be classified as serious games. These are defined by David Whatley as any game that is "not specifically entertainment, but which uses entertainment or the techniques and processes of the entertainment business, to achieve a purpose" [154]. Serious games have been developed for domains including learning, public policy, social activism, therapy, business, and health and safety. One commonly cited serious game is America's Army [146]. Falling within the genre of first-person shooter games, every stage of the development of America's Army was made in co-operation with the United States Army. The game reflects army culture and values, and functions as a recruitment tool [90]. America's Army is freely distributed, and targeted towards an audience of young American gamers. Similar in theme are two other games funded by the US military. Based on adapted off-the-shelf game technology, Virtual Iraq and Tactical Iraqi are targeted at soldiers. Virtual Iraq is a Virtual Reality environment in which the aim is to represent traumatic combat situations, in order to develop healthier trauma coping mechanisms among combat veterans suffering from Post-Traumatic Stress Disorder [73]. Tactical Iraqi is a languagelearning game designed to teach soldiers how to speak Arabic in the particular context of combat readiness [72]. In this interactive story-based 3D game, the player controls an avatar, Sergeant John Smith, and must carry out communication and negotiation with other characters in Arabic, within the context of a "socially realistic" combat situation. Given that both Virtual Iraq and Tactical Iraqi emphasise learning in a "real" context, these games are not just teaching language and stress coping skills. They are communicating a perspective on current world politics based on that of the US military.

Forming a separate field of research in its own right, the area of serious games has been contributed to by academics including Ben Sawyer [124], James Paul Gee [53], and Henry Jenkins [76]. Ian Bogost, editor of the online forum Water Cooler Games [25], has also written extensively about games that persuade, but positions himself apart from the serious games (and persuasive technology) community. Bogost suggests procedural rhetoric as the key rhetorical device games have over other forms of media, and as an alternative term for serious games, he proposes persuasive games, which he defines as "videogames that mount procedural rhetorics effectively" [24]. 
Within the serious games community, no consensus has been reached on how to measure the effectiveness of serious games on attitude change. Intuitively, it seems that games as persuasive technology hold much promise for changing people's attitudes: games are by nature interactive, and people tend to retain more impressions and be more swayed by arguments that engage them more deeply [113]. In comparison to more static forms of media at least, games are likely to be more appealing to younger generations, and an effective form of concisely representing real world information in a potentially entertaining manner.

\subsubsection{Advergames}

Games with a brand promotional purpose fall under the category of advergames, "the use of interactive gaming technology to deliver embedded advertising messages to consumers" [32]. Advergames are increasingly being used in marketing campaigns, both as supporting, and main channels of promotion. A good example is a game developed by the Brazilian Internet Service Provider (ISP) Telefonica. It requires players to yell into their computer microphones to wake up the game's protagonist, who has been using discounted night-time connection rates from a rival ISP [140].

As discussed earlier, while attitudes remain split over how to measure the effectiveness of serious games, some consumer psychology-based researchers have attempted to explicitly measure the effects of advergames. A German study examined people's attitudes towards advergames and their ability to recall brands promoted within them [136]. After play, $80 \%$ of the study participants were able to recall the promoted brand, while $50 \%$ claimed that after playing the game, they felt more positively about the brand. More than half of the participants passed the game on to their friends. One third of them did so voluntarily by email, thus activating viral marketing as a promotion strategy [136].

Other researchers investigating the impacts of advergames include Michelle Nelson et al., who focused on player attitudes towards advertising within games [102]. They found that people were generally positive about advertising content, and that those who disliked it disliked advertising in general. Lisa Winkler and Kathy Buckner also investigated people's acceptance and recall of product placements within games, and found that players demonstrated a high level of recall, especially when they already had some degree of familiarity with the brand being promoted [158]. Findings of this type demonstrate that it is possible to develop games that people like, remember, and find persuasive. 


\subsubsection{Discussion}

At present, the persuasive technology, serious games, and advergames literature lacks the accommodation of culture as a mediating factor in what people find persuasive.

Although Bonanni and Cyr are investigating the relationship between culture and e-loyalty, their research is too specific to the domain of online trust for it to inform general PT design [26]. The STOMP project has been promising in terms of results, but relies only on language cues to appeal to Māori audiences [28]. Grimes and Grinter discussed cultural relevance as a guiding factor in designing health technology for African American communities, but their work is still in early stages [57].

In terms of games, while many of the games we discussed touched on notions of culture, none have been motivated by the belief that cross-cultural differences may impact on the interaction and interfaces of games. Their designers designed with certain player audiences in mind, so these target audiences shaped the resulting games. We should note that to date, these games have tended to be developed in Western settings, destined for players of Western origin. There is an inherent cultural skew in favour of Western perspectives in current games that persuade.

The persuasive technology and games literature lacks both a theory for how culture may incline us to perceive persuasive technology, and guidelines for how we should design such persuasive technology to maximise its effectiveness for a specific target audience.

\subsection{Culture}

\subsubsection{Definitions}

Culture is a complex phenomenon, and this complexity is reflected by the many definitions proposed to describe it. One well-known definition of culture proposes:

Culture consists of patterns, explicit and implicit, of and for behavior acquired and transmitted by symbols, constituting the distinctive achievement of human groups, including their embodiment in artifacts; the essential core of culture consists of traditional (i.e. historically derived and selected) ideas and especially their attached values. [88]

From the wide range of definitions, two similarities that most researchers studying culture accept are that culture is acquired and transmitted, and that 
culture governs expected behaviour. It provides rules for how to behave.

One way to group definitions of culture is to identify whether the author considers culture to be externalised or internalised. In externalised views of culture, customs and traditions are the culture, and to study them is to study the culture itself. In internalised views of culture, culture is interlaced with the very thoughts of the individual, and these thoughts in turn influence behaviour. The behaviour then leads to culture-relevant customs and traditions.

The definition of culture we developed is:

Culture consists of shared learned patterns of beliefs and behaviours that govern how an individual interacts with others and the environment.

Crucial elements of our definition of culture are that it is a shared phenomenon, it is internalised, and that it shapes beliefs and behaviours.

\subsubsection{Cross-cultural comparisons}

In the $\mathrm{HCI}$ domain, cultural dimensions, aspects of culture that can be measured relative to other cultures [69], have proved to be popular tools for reasoning about culture and technology. A number of researchers have proposed different sets of cultural dimensions ${ }^{1}$, but those proposed by the sociologist Geert Hofstede, the cross-cultural psychologist Harry Triandis, and the anthropologist Edward Hall have been particularly embraced by the HCI community. In this section, we introduce their work, and also overview the research of Shalom Schwartz. His work has been underused within $\mathrm{HCI}$, while being influential in the cross-cultural psychology literature.

\subsubsection{Geert Hofstede}

Between 1978 and 1983, Geert Hofstede surveyed hundreds of IBM employees on their attitudes relating to work in 53 countries and determined patterns of similarities and differences in their responses. In the book Cultures and Organisations: Software of the Mind, Hofstede discusses five dimensions that apply at the cultural level, including Individualism - collectivism: whether society members have a primarily individual or an interdependent identity, and Power distance: whether bosses and subordinates consider themselves to be social equals [69]. In our research, we have relied on the individualism - collectivism dimension as a theoretical grounding, so we discuss this dimension here in more detail. We also discuss the closely related dimension of power distance, which was initially considered

\footnotetext{
${ }^{1}$ We note that the term cultural dimensions was coined by Geert Hofstede [69].
} 
as part of the individualism - collectivism dimension in Hofstede's original publication of this research, and co-varies with it inversely [68]. Nations that score high in individualism are likely to score lower in power distance, whereas nations that score low on individualism are more likely to score higher in power distance.

\section{Individualism - collectivism}

Individualism pertains to societies in which the ties between individuals are loose: everyone is expected to look after himself or herself and his or her immediate family. Collectivism as its opposite pertains to societies in which people from birth onwards are integrated into strong, cohesive ingroups ${ }^{2}$, which throughout people's lifetime continue to protect them in exchange for unquestioning loyalty. [69]

In the extreme individualist society, the interests of the individual outweigh group interests. The typical family structure is that of a nuclear family, and children are raised to think of themselves as " $\mathrm{I}$ " and voice their own opinions. Often they are self-motivated, goal-oriented, and use guilt and loss of self-respect as motivators. The most individualist societies are the USA, Australia, and Great Britain [69].

In the extreme collectivist society, the interests of the group outweigh individual interests. The family structure is typically an extended family, and children are raised to think of themselves as "we", respect their elders, and taught to owe lifelong loyalty to their ingroup in exchange for constant protection. Often they strive to maintain social harmony, develop skills and virtues necessary for being a good group member and maintaining tradition, and use shame and loss of face as motivators. The most collectivist societies are Guatemala, Ecuador, and Panama [69].

\section{Power Distance}

...the extent to which the less powerful members of institutions and organisations within a country expect and accept that power is distributed unequally. [69]

In low power distance countries, as a child grows up, her relationship with her parents turns into a relationship of equality, as opposed to remaining a strictly parent-child relationship. At schools, students are encouraged to engage with

\footnotetext{
${ }^{2}$ An ingroup is a group of people about whose welfare a person is concerned and separation from whom leads to anxiety [142].
} 
teachers. In the workplace, while the organisational hierarchy may create relationships of inequality through the boss-subordinate relationship, the bosses and subordinates feel existentially equal. The three lowest power distance countries in Hofstede's study were Israel, Denmark, and New Zealand [69].

In high power distance countries, children never outgrow the parent-child relationship, as parents retain authority as long as they are alive. Furthermore, these countries have tacit respect for elder society members. The same pattern is observed at school, and in the workplace. Malaysia, Panama, and Guatemala were ranked as the highest power distance countries [69].

\subsubsection{Harry Triandis}

Harry Triandis's research is grounded in psychology, and has formed a cornerstone of the cross-cultural psychology literature. Triandis has focused attention on a range of cultural dimensions, but one of his most notable contributions is his work on individualism and collectivism, published after Hofstede's seminal work [68]. Triandis's views on the basic characteristics of individualism and collectivism are in keeping with Hofstede's views. Triandis also adds that individualists exhibit more attitude-behaviour consistency, perform their duties if it is advantageous in terms of benefit, and that their self-identity is defined independently of specific collectives. Collectivists on the other hand exhibit less attitudebehaviour consistency, enjoy doing "what is right" for their collective, and have self identities that are strongly linked to attributes of their ingroup [142].

A key difference between Triandis and Hofstede's views of individualism and collectivism is that Triandis views them as two distinct, yet related, categories, as opposed to being two ends of the same continuum. Additionally, Triandis performed his studies at an individual level of analysis, rather than at a cultural level. Furthermore, Triandis adds another level of cultural classification to his model of individualism and collectivism: he identifies the horizontality - verticality construct. This is related to Hofstede's power distance dimension, in that a horizontal quality emphasises equality, whereas a vertical quality emphasises hierarchy [144]. The combination of individualism and collectivism, alongside horizontality and verticality, results in the creation of four distinct groups: Vertical Individualism (VI), Horizontal Individualism (HI), Vertical Collectivism (VC), and Horizontal Collectivism (HC). Where Hofstede's research assumes that countries are mono-cultural, Triandis's categorisation does not exclude the possibility of multiple cultures existing within the same country. 


\subsubsection{Edward Hall}

Edward Hall is an anthropologist whose work has been influential in cross-cultural studies grounded in a range of disciplines. Like Hofstede and Triandis, Hall proposed theoretical frameworks for categorising culture, which relate to individualism and collectivism [62]. One of these differentiates world cultures according to context, that is, low-context and high-context. Low-context cultures share common characteristics with individualist cultures: bonds between people are looser, communication tends to be explicit and verbal, knowledge is codified, public, external, and accessible, and people play by external rules. High-context cultures effectively describe collectivist cultures: bonds between people are stronger and last for longer durations, communication is more implicit and often non-verbal, knowledge is situational, and there are strong ingroup and outgroup ${ }^{3}$ boundaries [62].

\subsubsection{Shalom Schwartz}

Intrinsically related to culture is the notion of value preferences: for example, Hofstede defines culture as "a system of collectively held values" [69]. From a background of social psychology, Shalom Schwartz conducted research in the area of value priorities and motivations. Schwartz defines values as "emotionlinked beliefs that represent desirable, transsituational goals or modes of conduct that promote these goals" [128]. Schwartz proposed a universal values continuum of ten value types: Power, Achievement, Hedonism, Stimulation, Self-direction, Universalism, Benevolence, Tradition, Conformity, and Security [128]. The values continuum, shown in figure 2.1, is circular in form, with adjacent sectors sharing underlying values, and opposite sectors representing conflicting values. The ten types were derived a priori from biology, sociology, anthropology, and psychology theory. Several studies have examined whether Schwartz's value constructs have the same meaning across cultural groups, and all have reported that the theory reasonably comprehensively captures distinct value types that are recognised across cultural groups $[47,128,130]$.

\subsubsection{Discussion}

Hofstede's and Hall's research applies at the cultural, rather than individual level. Both provide descriptive frameworks for typical attitudes and beliefs of people originating from certain types of cultures. In comparison, Triandis's and Schwartz's

\footnotetext{
${ }^{3}$ The outgroup is defined as anyone who is not a member of the ingroup $[69,96,142,143]$.
} 


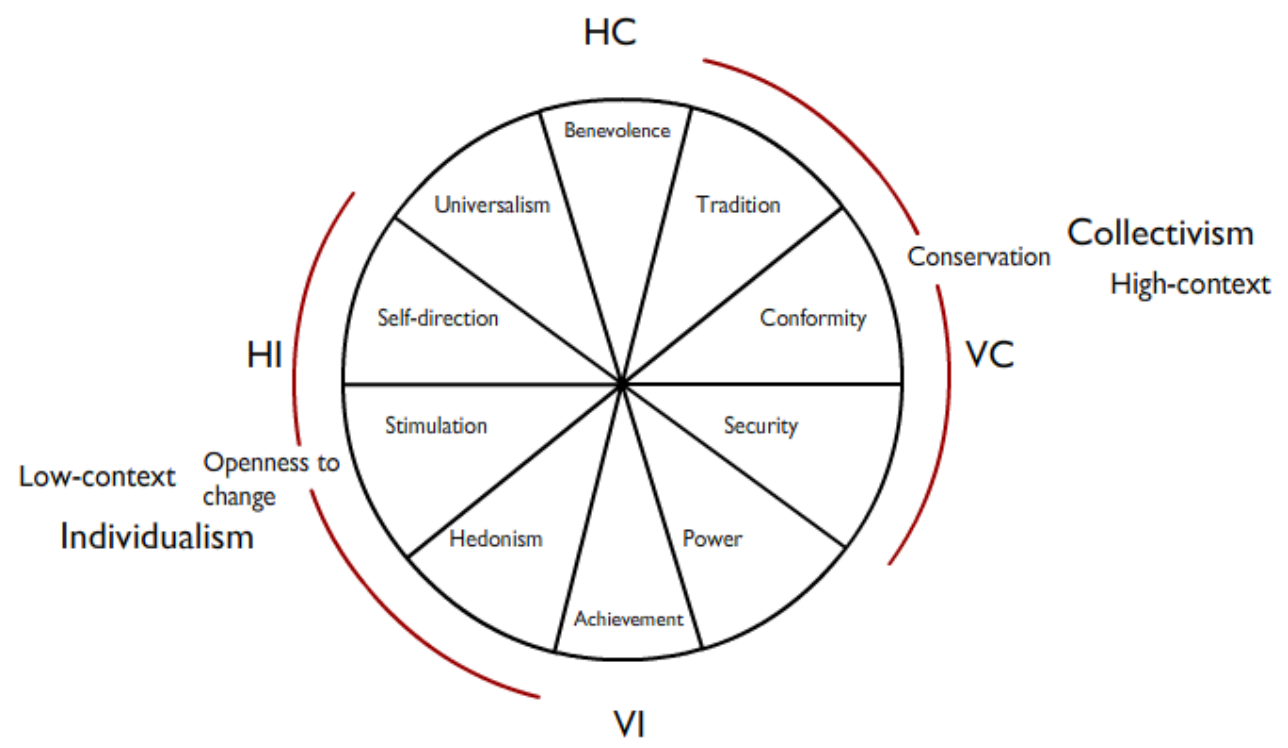

Figure 2.1: Schwartz's values continuum with cultural dimensions

research applies at the individual, rather than cultural level, although both levels are conceptually linked $[2,144]$.

Hofstede's work has arguably been the most successful in terms of mainstream adoption: his dimensions have been used by people from a range of disciplines, including psychology, HCI, advertising, business, and education. In recent years, it has faced some criticism, however. For example, the dimensions are applied at a national level, and are premised on an assumption that countries are mono-cultural, or that most people in any given country share the same culture. Another issue with Hofstede's work is that because the survey was conducted only with IBM employees, it might have identified values specific to the IBM workers, who were predominantly middle class. Further, Hofstede assumed that the IBM "culture" around the world is identical, and that differences observed between one IBM employee in one country and another IBM employee in another country must be the sole product of national differences [98].

In spite of these issues, individualism and collectivism (and indeed, Hall's related classifications of low-context, and high-context cultures) provide a powerful theoretical categorisation for reasoning about general differences between types of cultures. They are also considered to be reasonably comprehensive: an analysis of Hofstede's IBM data showed that individualism and collectivism (along with power distance) accounted for the majority of the data variance [68, 143]. Additionally, individualism and collectivism have been investigated from different (but related) perspectives by a number of researchers, for example, Triandis [143], Hazel Markus and Shinobu Kitayama [96], and others. These dimensions seem particularly useful for investigating the relationship between culture and 
persuasion $[2,38]$. Much of the recent cross-cultural consumer research involving cultural dimensions has focused on individualism and collectivism, whether it be Hofstede's version, or another researcher's equivalent.

In contrast to the work of Hofstede, Triandis, and Hall, which is concentrated on culture, Schwartz's research has concentrated strongly on values. Schwartz does however discuss a bipolar dimension inherent in his continuum: Openness to Change values (Self-direction, Stimulation, and Hedonism) define one end, and Conservation values (Tradition, Conformity, and Security) define the other [129]. Openness to Change and Conservation values share conceptual ground with individualism and collectivism. Figure 2.1 shows a conceptual mapping between the cultural dimensions and value theory we have discussed thus far.

A noteworthy point of difference between concentrating on cultural dimensions and values is that dimensions focus on preferred or prevalent states of behaviours [69], e.g. prioritising group goals, while values are preferences for beliefs about desirable modes of conduct [14], e.g. conformity. While this means that for a values focus, situational variables have less impact on individual responses, the correspondence between people's values and people's behaviour is not always obvious.

\subsection{Cultural aspects of $\mathrm{HCI}$}

Over the last couple of decades, international software sales have increased dramatically $[40,159]$. Drivers for this increase include a fuller adoption of information technology, the globalisation of the market place, the proliferation of internet use, and the growth of e-commerce. The international software market is now facing more competition, and many exporters are beginning to develop localised versions of software for their international markets, such as China and India, which represent huge revenue potential [159]. Initially, the localised versions of software dealt with common-sense cultural conventions, such as language translations of interfaces, time zones, date formats, and currency forms [40, 104, 123].

Culture and its effects clearly run more deeply than these conventions [69]. The study of cultural aspects of human-computer interaction is a growing area of research that focuses on the interplay of culture and people's interactions with technology.

\subsubsection{Covert culture}

In HCI, the 1990s signalled the beginning of the shift from concerns about surfacelevel, or overt conceptions of culture to deeper embedding of culture in interfaces, 
or covert culture. Alvin Yeo's Cultural User Interfaces (CUI) conceptual model is one example of such research [159]. Yeo proposed the development of interchangeable CUIs for software, where each CUI would in effect be context-aware of the culture users had specified for themselves. Users could have multiple CUIs, representing membership of multiple cultures and subcultures. Other researchers have taken ethnographic approaches to uncovering covert culture. Albert Badre and Wendy Barber investigated localised cultural preferences across different genres of websites by conducting "foraging studies". In the foraging studies, they examined websites to identify patterns of interface design elements and features, which they called "cultural markers" [13, 16]. Badre and Barber then performed usability tests by creating hypothetical pages featuring the identified cultural markers, and found that target culture users did prefer pages featuring their specific target cultural markers over others.

\subsubsection{Cultural metaphors}

User interface metaphors have also been investigated from the cross-cultural perspective. Vanessa Evers researched the cross-cultural applicability of the virtual campus metaphor [40]. Her study involved testing icon and label recognition and overall metaphor comprehension among American, British, Dutch, and Japanese students. She found that the virtual campus metaphor was problematic because different cultural groups associated different meanings to the same objects. Her study also revealed issues in suitability of user evaluation methods on different user groups: British and Japanese users in particular felt uncomfortable performing think-aloud tasks (which we overview in upcoming \$3.4.2.3). Informed by an experiential perspective, Marion Walton and Vera Vukovic recounted experiences of teaching in South Africa, and discussed the fallacy of the notion of the information highway connecting the entire world [149]. They claimed that vast cultural differences made it difficult for South African students to transition to web use, the reason being the often overlooked fact that web use is situated within a distinct and elite reading and writing culture.

\subsubsection{Cultural dimensions}

Over the last decade, cross-cultural psychology models of culture have been employed to guide culturally-focused HCI investigations. Hofstede's dimensions of culture have been the most frequently used model in the HCI literature to date [69], followed by the models of Hall [62] and Triandis [141]. One reason for the dominance of Hofstede's dimensions in the HCI community might be 
that enough other practitioners have used them that they have become the de facto standard. A secondary explanation may be that Hofstede's five dimensional model seems to offer a more complete picture of how cultures differ than do other dimensional models.

An example of Hofstede-based HCI research that ties into our own research interests is Thomas Vöhringer-Kuhnt's investigation into the influence of culture on usability [148]. Vöhringer-Kuhnt hypothesised that people of different cultures would perceive differently the effectiveness, efficiency, and satisfaction of use of the IBM website. After a web-based survey regarding the usability of the IBM website, he found that Australians and South Africans focus more on efficiency and satisfaction than on effectiveness, while Americans and Europeans prioritise goal and task completion. Overall, Vöhringer-Kuhnt managed to find support for the idea that individualism - collectivism is correlated to differences in usability perception, but was unable to show that all of Hofstede's cultural dimensions impacted on perceptions of usability. Similarly concerned with which cultural dimensions actually impact usability, Aaron Marcus and Valentina-Johanna Baumgartner presented the results of a survey conducted on 57 usability experts around the world, regarding which cultural factors they believed had the largest impact on perceptions of usability [94]. The results showed that the five most important cultural factors were believed to be context (which can be high-, low-, or middle-), relationship to the environment and technology, level of technological development, time perception, and uncertainty avoidance. Nearly all of the survey participants, however, said that "everything depends" on the purpose of the UI itself and the user domain. Other dimensions-based HCI research includes Aaron Marcus's analysis of websites in the context of Hofstede's dimensions [95]. For each dimension, Marcus examined the websites of countries classified on the low and high ends of the dimension respectively. He highlighted differences in content presentation that were in keeping with the specified characteristics of the extreme ends of each of the five cultural dimensions. Marcus's work was exploratory in nature, and he did not explicitly seek out examples of contradictory cultural characteristics within the websites. It has, however, been influential on other HCI research, as it represents one of the earliest HCI adoptions of cultural dimensions.

\subsubsection{Discussion}

Culturally-focused HCI literature lacks at present a focus on the relationship between culture and persuasive tools, where the key objective is to change people's attitudes or behaviours by use of the product. While researchers such as 
Vöhringer-Kuhnt and Marcus have shown how cultural dimensions research can be repurposed to explain differences in technology perceptions [95, 148], research of this type has tended to focus on productivity applications over persuasive ones. Productivity applications primarily serve to facilitate particular types of productivity, so productivity enhancement is the main focus. There is no overt attempt to shape people's attitudes through application usage. Instead, researchers are investigating how best to support users from a range of cultures with regards to achieving productivity-focused tasks.

Persuasive technology is aimed at changing people's attitudes through technology usage: productivity is a focus but largely in a supporting role to attitude and behaviour change. An explicit indicator of how culturally-focused HCI does not address persuasion as an important variable is in the evaluation techniques researchers use. In conducting evaluations, the culturally-focused HCI researchers do not use techniques to evaluate attitude change as a consequence of tool usage.

\subsection{Cross-cultural psychology and consumer behaviour}

The significance of the relationship between culture and persuasion has been explored for some time in the field of cross-cultural psychology, or global consumer behaviour. In part, this interest has been fuelled by the globalisation of markets, and competition to successfully promote particular products in different cultural settings. For example, Marieke de Mooij's research has focused on high-level conceptions of convergences and divergences of consumer behaviour across countries [38]. Her view is that all aspects of consumer behaviour are affected by culture, and understanding how this process is taking place is crucially important in maximising marketing efficiency. From a more fundamental, and psychologydriven perspective, questions underlying much cross-cultural consumer research include whether North American-derived theoretical frameworks for communications strategies are exportable to other countries, and whether we can derive generalised theoretical frameworks to describe global consumer behaviour [93].

The research capability in the cross-cultural consumer psychology domain is large, motivated in part by potential profits. Accordingly, researchers have investigated a range of phenomena, much of which has used individualism and collectivism as a way of differentiating between cultures [4]. We summarise some of the consumer psychology literature here that reflects our interest in the individualism - collectivism dimensions. 


\subsubsection{Cultural styles in advertising}

Advertising across cultures has been a topic of interest for several cross-cultural consumer researchers, as advertising is a clear example of persuasive communication. Sang-Pil Han and Sharon Shavitt investigated differences in advertising styles between the USA and Korea [63]. Sampling a wide range of advertisements, they found that American advertisements feature more individualistic appeals, while Korean advertisements feature more collectivistic appeals. The second part of their study involved developing their own advertisements. Han and Shavitt found that members of individualist cultures found individualistic appeals to be more persuasive, while members of collectivist cultures found collectivistic appeals to be more persuasive. Daeschun An also focused on cultural influences in advertising, and used Hall's and Hofstede's dimensions to develop a framework to identify and explain differences in information cues and strategies used in Korean and American internet advertising [10]. After analysing 600 advertising websites, An found that Korean advertisements tend to reflect a more highcontext, collectivist, polychronic, feminine, and high-uncertainty-avoidance culture, while American advertisements reflect an assertive, low-context, personalspace, highly individualist, high-power-distance, and low-uncertainty-avoidance culture.

\subsubsection{Individualism, collectivism, and persuasion}

The effects of individualism and collectivism on persuasion have been explicitly investigated by Jennifer Aaker and Durairaj Maheswaran, who developed experiments to uncover culturally-driven persuasion differences [4]. Relying on dualprocess theories of persuasion [113], Aaker and Maheswaran investigated how individualists and collectivists responded to persuasion attempts under both low elaboration routes of persuasion, where people are for some reason unable to make considered decisions, and high elaboration routes of persuasion, where people are able to make decisions thoughtfully, and feel motivated to do so. They found that under conditions of high elaboration, collectivists place more importance on consensus than do individualists.

Culturally influenced beliefs have also been a subject of investigation for Aaker, who conducted experiments designed to reveal whether people of different cultures react to advertising appeals based on how closely they matched cultural norms of attitude and emotion patterns [6]. She established that individualists tend to recognise and prefer ego-focused appeals, which reference internal states of being, whereas collectivists tend to recognise and prefer other-focused appeals, 
which contextualise appeals in terms of their ingroup members.

Aaker and Williams also investigated the impact of other cultural stereotypes [157]. An important facet of East Asian collectivism concerns the acceptance of dual realities, e.g. the coexistence of good and bad, or happiness and sadness. In contrast, people of Western cultures have been more likely to reject dual realities. In experiments featuring American and East Asian participants, Williams and Aaker showed that this philosophical difference was again borne out in people's responses to advertising. Americans had less favourable attitudes towards mixed emotion persuasive appeals than East Asians.

Aaker and Schmitt looked at the need for self-expression across individualist and collectivist cultures, and showed that people from both were concerned with self-expression, but the manifestation of the type of self-expression differed [5]. While their American test participants were more concerned with self-expression as a point of differentiation between themselves and their peers, the Asian test participants were more interested in self-expression as a means of showing how they assimilated with their peers.

Also focusing on individualism and collectivism differences, Gurhan-Canli and Maheswaran examined the effectiveness of comparative advertising across individualist and collectivist populations [61]. Although comparative advertising is not common in Asian countries, Gurhan-Canli and Maheswaran managed to demonstrate that comparative claims based on showing how one brand is similar to another were more effective on Asian populations than superiority-based claims, that showed how one brand is inferior to another. They found the reverse effect on their American test population.

\subsubsection{Stability of cultural preferences}

A key question underlying some of the cross-cultural consumer research is whether cultural differences always play an important role on consumer attitudes. As a partial solution, Aaker looked into how and whether the effectiveness of advertising appeals to audiences of particular cultures was caused more by diagnosticity (i.e. importance) or accessibility (i.e. ease of immediate comprehension), because cross-cultural psychology research indicates that different cultures are more swayed by certain types of appeals than others [1]. Using a dual-process theory of persuasion [113], Aaker found that under conditions of low elaboration, accessibility of appeals impacted persuasion effects on people. Under high elaboration, on the other hand, accessibility has less impact, and cultural differences were muted.

Donnel Briley and Aaker showed that people's reliance on cultural beliefs and 
attitudes was related to how much time they perceived they had to make judgements [29]. Under conditions of high pressure, regardless of cultural orientation, people were more likely to fall back on cultural knowledge and beliefs to guide their decisions. Under low time pressure, in which people had more time to deliberate and reflect on their personal experiences, they were less likely to be guided by culturally prescribed norms. Interestingly, though, Briley and Aaker discussed how there was probably a continuum between the low- and high- pressure situations, and that persuasive mediums requiring lower levels of attention to process might benefit the most from the extensive use of cultural cues.

In spite of Hofstede's claims that cultural differences are stable and long term [69], a recent content analysis study by Jing Zhang and Shavitt indicated the beginnings of a different trend [163]. After analysing magazine articles targeted at the largely urban, affluent, and English-speaking Chinese generation X, Zhang and Shavitt found that modernity and individualism values outnumbered more traditional Chinese cultural values. At the same time, they found that television commercials targeted at the mass market were still dominated by collectivism and tradition values. We note that Zhang and Shavitt did emphasise that advertising has previously been perceived as a revolutionary force. They discussed how the general advertising emphasis on new products and ideas means that advertising may be less reflective of the culture in its present state, and more reflective of how the culture might evolve [120].

\subsection{Limitations of the current approaches}

Our discussion until this point has demonstrated that there is no shortage of research conducted on persuasive technology or culturally-focused HCI. Unfortunately, neither body of research contains literature that focuses in depth on applications concerned with both persuasion and culture.

Persuasive technology researchers, at present, have not treated culture as an important factor in what users find persuasive, and for determining the nature of the persuasion appeals embedded in PT designs. Successful persuasion usually needs to contain appeals that are of relevance to target audiences [38, 152]. The cross-cultural consumer behaviour research provides evidence that culture plays a key role in determining the nature of these appeals, as the appeals need to integrate with culturally-influenced attitudes and beliefs [6, 10, 38, 63]. Research findings also show that persuasive tactics and strategies that work effectively on audiences of one culture may not work well on audiences of different cultures $[5,61,63,157]$. 
The lack of focus on culture does not mean that the present PT literature is culturally-neutral, however. Much of the literature on persuasive technology originates in countries classified as individualist, and discusses and examines products developed for individualist audiences. The cross-cultural consumer psychology findings have demonstrated on numerous occasions that individualist and collectivist consumers respond differently to the same appeals. Thinking about these differences from a broader context of persuasion (as opposed to consumer persuasion), it is not unreasonable to guess that persuasive technology originating from individualist countries reflects a bias towards individualist cultures. Furthermore, the favouring of individualist culture motivations in persuasive technology may in fact lead to reduced effectiveness on users of collectivist cultures.

HCI researchers, in comparison, have for some years now been investigating the role culture plays on interface usability and perception. They have tended, however, to focus on aspects of productivity applications. Typical productivity applications and persuasive technology differ in a fundamental manner. Productivity applications are designed with the goal of facilitating the user in completing particular types of tasks. Designers are not intentionally setting out to effect attitude and behavioural changes in users. This is not the case for persuasive technology. Here the facilitation of productivity is important because of the attitude and behavioural changes it supports.

As evidence that persuasion has not been a goal of culturally-focused HCI researchers, the evaluation methods used in culturally-focused HCI research do not measure attitude or behaviour change resulting from technology usage.

The lack of a useful intersection between persuasive technology and the culturallyfocused HCI calls for a research approach that recognises not just the inherent linkages between culture and persuasion, but the importance of factoring in culture in the research of persuasive technology. 


\section{Chapter 3}

\section{Methodology}

As a research domain, persuasive technology draws heavily on theory, given its grounding in social, and consumer psychology. Persuasive technology is also strongly rooted in practice, as it concerns the design, development, and usage of technology. In investigating persuasive technology, then, it is important to focus on both its theoretical and practical aspects. In this chapter, we begin by establishing a theoretical justification for our research focus. We then discuss the underlying rationale of our research methodology, and the processes employed.

\subsection{Overview}

\subsubsection{Culture and persuasion}

In §2.2.2.3 we introduced Hall's views on culture. To quote Hall in Beyond Culture:

What makes it doubly hard to differentiate the innate from the acquired is the fact that, as people grow up, everyone around them shares the same patterns. [62]

The cultural unconscious, like Freud's unconscious, not only controls man's actions but can be understood only by painstaking processes of detailed analysis. [62]

Culture is a pervasive factor in day-to-day life, and by extension plays a part in shaping the design preferences of PT designers, and the strategies they use and embed in their products. In making design decisions about persuasive technology, designers are constantly making culturally-based value judgements about what they personally find persuasive, what they believe their target audience finds persuasive, and which persuasion motivations they believe are worthwhile 
mobilising within the PT design. Sometimes designers make these decisions consciously, and at other times subconsciously, but undoubtedly, decisions are always made.

While it may be exceptionally difficult for designers to identify cultural preferences that they have unknowingly "embedded" into their technologies, these ideals and values will resurface when the technologies are used by audiences the designers were not considering. As Hall explains:

The only time one is aware of the control system is when things don't follow the hidden program. This is most frequent in intercultural programs. [62]

Hall was referring here to the hidden internalised cultural programs people operate with, that form integral parts of each of our personalities [62]. At the same time, his words resonate with anyone who has used technology and felt dissatisfied because they felt the technology was premised on mismatched assumptions about their identity, their knowledge base, their behaviour, or their typical interaction patterns. This response should concern PT designers, as an intended outcome of persuasive technology use is essentially small-scale personality change, by way of incorporation of different attitudes and behaviours.

One solution for dealing with the user-product mismatch is to ignore it. While this is the easiest workaround, and currently what is widely practised, this solution is riddled with difficulties. Aside from its ethnocentrism, we must question how successful it will prove as a long term solution. For example, by supplying PT users of collectivist cultures with a PT designed for users of an individualist culture, we are effectively asking the users to oppose their cultural beliefs temporarily to accommodate the technology. We are then further asking them to become persuaded based on this cultural belief shift. Even if, through repeated exposure, the users begin to permanently adjust their cultural beliefs, this is not an efficient solution. We propose that it is more effective and easier to persuade people within the frameworks of their own culture than to instigate in them a large-scale cultural change in order to persuade them.

The other solution is to recognise that cultural differences exist between user populations, and to actively investigate how the populations are different. In establishing these differences, we are more likely to be able to identify the best ways to connect with desired target audiences because the design decisions driving PT development will be more culturally informed. Referring again to Hall's notion of the "hidden program", if the cultural assumptions of a PT match the cultural assumptions of its users, their attention is freed up to focus on the non-cultural, 
informative content related directly to attitude/behaviour change. In short, this increases the potential effectiveness of persuasive technology.

Until now, the technology at the intersection of persuasive technology, culturallyfocused HCI, and cross-cultural psychology has not been clearly identified. As it is fundamental to this research, we will refer to it as culturally-relevant persuasive technology, which we define as:

Interactive computing products created for the purpose of changing people's attitudes or behaviours, that reflect the cultural motivations of their target users.

Within this context, our research hypothesis is:

Persuasive technology is more effective when it reflects the culture of its intended target audience.

\subsubsection{Research design}

We arrived at the need to research culturally-relevant persuasive technology through a process of inductive reasoning. No prior theory existed to support the value of culturally-relevant persuasive technology, and we were interested in initiating such a theory. As well as focusing on theory, however, we wanted to design, develop, and evaluate a culturally-relevant persuasive technology.

Qualitative study approaches have traditionally been used to explore areas that are under-researched, and where there is little supporting theory $[35,36]$. The researcher assumes an objective stance to the ontological issue for what is real, and acknowledges that her views will be value-laden and subjective. Furthermore, the researcher interacts directly with the subjects of the research, with the objective of drawing on inductive reasoning to synthesise patterns or theory about the phenomena under investigation.

The methods of data collection that we foresaw as helpful for developing theory, namely, interviews and focus groups, are qualitative in nature. They require interaction with participants, and an awareness that data interpretations are value-laden.

Quantitative approaches, on the other hand, fit into a more positivist worldview [35]. They are typically used to investigate areas supported by a rich body of research. The researcher works on the assumption that reality is objective and singular, and that she is able to separate her own values from what is being researched. As data is readily accessible for analysis, the goal of the researcher is usually to identify data generalisations to aid prediction, explanation, and understanding. 
Usability-focused HCI investigation often relies on quantitative methods (e.g. $[105,125])$, that is, computers and technology are largely treated as objective phenomena, and researchers seek to produce generalisable findings about them. Persuasive technology as a domain concerns not just technology, but also psychologyoriented attitude/behavioural change, which also tends to be evaluated using quantitative measures (e.g. [6, 38, 63]).

As no one paradigm suited our purposes, and there seemed to exist equally relevant reasons for our investigation to be both qualitative and quantitative, we adopted a mixed-methods approach in our research [35] that combined both approaches.

\subsubsection{Research phases}

In spite of the abundance of research focusing on differences between individualist and collectivist cultures, there is little literature guiding the design of culturallyrelevant persuasive technology. To explore this issue, we wanted to determine whether culturally matching tools with audiences resulted in more effective persuasion. Specifically, we decided to develop design concepts for culturally-relevant persuasive technology, to trial the design concepts in two versions of a PT prototype, and then to evaluate whether the two versions of the prototype would be perceived differently by a target and a non-target audience.

To meet these three study objectives, we structured our investigation in three phases, each using distinctive methods and techniques. We discuss each research phase and its associated methods separately. We deal with the initial theory development phase in $\S 3.2$, the prototype development phase in $\S 3.3$, and the evaluation phase in $\S 3.4$.

\subsubsection{Researcher bias}

In qualitative research, the biases and assumptions of the researcher must be acknowledged up-front. As our research is partly qualitative, we present here such a description of the key researcher. The statements about games and smoking towards the end of the description will be explained further in this chapter.

I am a twenty five year old middle-class female, born in Canada, of Bangladeshi ethnic origin. I spent the first two years of my life in Hawaii and moved to New Zealand at the age of two. I grew up in a household with two parents, and one elder sister, in which traditional Bangladeshi values were emphasised. Everyone in my imme- 
diate family, including myself, is bilingual (Bangla and English), and comfortable with both Bangladeshi and New Zealand culture.

Education has always been highly valued in my family: my father is a university lecturer, my mother is a school teacher, and my sister is a medical doctor. To date, all of my education has been completed in New Zealand.

I am comfortable using technology, having grown up around computers, and given my bachelors degree in Computer Science. With regard to video or computer games, however, I am significantly less experienced. Before undertaking this research, I did not have much exposure to video or computer games, or the game design process.

I am not a smoker, and neither is anyone in my immediate family. In my household, it was made clear to me from early on that smoking was an unacceptable behaviour.

All of the details disclosed influence my interpretations as a researcher.

\subsection{Theory Development}

\subsubsection{Objectives}

The domain of cross-cultural consumer psychology, which shares obvious links with the field of persuasive technology, has as yet remained untapped by PT researchers. As we claimed in $\S 2.5$, such a literature should be developed, especially as the culturally-focused HCI literature does not address attitude change as a key objective. In addition, it seemed apparent that with some repurposing, we would be able to harness the findings of the cross-cultural psychology literature on individualism and collectivism to support the notion of culturally-relevant persuasive technology.

In his 2003 book, Fogg outlined a set of strategies that he claimed were prevalently used in PT tools at the time of publication. This approach somewhat mirrors the motivations underlying the pattern movement. First popularised by Christopher Alexander in the late 1970s as a means of capturing time-honoured architectural design solutions [9], patterns were later adopted by the software engineering community. In the guise of "design patterns", they were used to describe how to develop building blocks, or micro-architectures, for solving larger problems [50]. While both Fogg's PT strategies and design patterns similarly concern the communication of existing design wisdom, patterns are also intended for use 
in guiding design solutions. That is, patterns are not simply descriptive of situations in which designers have used particular solutions, they are also operational. They describe solution application, and the problems resolved, as a result.

A key motivation driving our research was to be able to guide the design of culturally-relevant persuasive technology. Thus, following the pattern concept, we set out to develop a set of culturally-relevant PT strategies that would aid designers in designing culturally-relevant persuasive technology for specific target audiences. Each strategy is structured in a standard form, consisting of Target audience, Antecedents, Description, Real world parallel, Hypothetical PT example, Consequences, Summary of key motivations, and Related strategies. The advantage to presenting each strategy in this manner is that they are readily usable for designers, in terms of coherence, ease of use, and comparison.

\subsubsection{Process and methods}

During the Theory Development phase, we used two methods to inform our research: semi-structured interviews and content analysis. We overview the technical aspects of each method below, and then discuss how we used the methods in our investigation.

\subsubsection{Interviews}

Overview Interviews are a commonly used method in qualitative inquiry, generally involving one-on-one discussion between an interviewer and a participant [36]. The role of the interviewer is to question the interview participant, or interviewee, about a particular topic. Traditional interviews usually consist of the interviewer questioning a participant according to a pre-determined script of questions. Semi-structured interviews, on the other hand, are more flexible in that interviewers work from a discussion outline containing broad questions, but might also venture off the interview script, using their own discretion to determine how to proceed. A disadvantage of interviews as a methodology is that interviewees may not be completely honest in their responses, and might tailor their responses to how they think the interviewer wishes them to respond.

Interviews with social marketing campaign stakeholders Designers of PT websites and services undoubtedly utilise implicit knowledge about persuasion strategies, and how they may be effectively used. To learn about how some designers approach the task of shaping persuasion appeals, along with their reasons for using various persuasive strategies, we conducted interviews with stakeholders 
involved in two NZ social marketing organisations, which we refer to using fictional names. One organisation, "Support", is a government-funded non-profit organisation focused on reducing smoking rates among the Māori population. The other organisation, "Future", aims to educate people about financial matters and retirement planning. For each organisation, we interviewed three people involved in campaign strategy or design, for roughly 60 minutes each, making audio recordings of each participant. We then made transcripts of each interview and began an iterative process of code allocation, code grouping and merging, and higher-level categorisation [36]. Once we reached a stage of categorisation convergence stability, we identified three themes that had emerged from the data, which we overview in $\S 4.1$. The interview findings also helped motivate several of our design strategies, which we cover in $\S 4.3$. The interview agenda is contained in appendix $\mathrm{C}$.

\subsubsection{Content analysis}

Overview Content analysis, a method commonly used in the social sciences, is "any technique for making inferences by objectively and systematically identifying specified characteristics of messages" [70]. Content analysis is used to make inferences about the antecedents, the characteristics, and the effects of a message [70]. Before commencing the analysis, the researcher makes a hypothesis about what she expects to find as a result of the analysis. The content, or text, is then studied for the purpose of identifying keywords. These keywords are next considered in the context of a larger theoretical framework relating to the hypothesis. In a more quantitative style of analysis, the keywords might be counted for fre-

quency, based on the assumption that the more frequently occurring keywords reflect the importance of a concept within the message. In a more qualitative style, the keywords might be organised into categories, from which the researcher can then make inferences about the overall text.

Content analysis of extant theory Our theory was informed by existing literature. The initial phase of theory development consisted of gathering materials from the cross-cultural psychology literature on the behavioural and motivational differences between individualists and collectivists. We also conducted a literature survey of cross-cultural consumer psychology research, with the aim of identifying trends of preferences for individualist and collectivist audiences. We then performed a conceptual textual analysis on Fogg's PT tool strategies, using as an analytical context the cross-cultural psychology literature on individualism and collectivism. We present the analysis as an examination of the motivations un- 
derlying Fogg's PT tool strategies. The examination we refer to is discussed in $\S 4.2$.

From this point, we were able to synthesise new culturally-relevant PT strategies, largely informed by the cross-cultural psychology and consumer psychology literature on individualism and collectivism, but also informed by the examination of Fogg's PT strategies, and findings from the interviews with social marketing stakeholders. The strategies we refer to here are detailed in $\S 4.3$.

\subsection{Prototype Development}

\subsubsection{Objectives}

To establish whether the culturally-relevant PT strategies we proposed would be practically useful in the design and development of a real PT tool, we decided to build a "proof of concept" tool from inception to completion.

In Chapter 2, we discussed some application platforms for which persuasive technology has been developed, including games. The literature connecting games and persuasive technology is sparse at best, and there are few helpful guidelines for how to design relevant and effective persuasive games. Therefore, we decided to use games as a format for our culturally-relevant persuasive technology.

Before we could progress with developing our PT game, however, we needed to decide which domain the game would fall under. We were interested in developing a game about a social marketing issue, and wanted the issue to concern both an individualist and collectivist audience.

Smoking While New Zealand ranks eighth lowest out of 30 OECD countries with regards to overall rates of smoking among the population, some of its subpopulations demonstrate high rates of smoking [100]. For example, $50 \%$ of Māori women classify themselves as smokers, while only $20 \%$ of NZ European women are smokers. The discrepancy between Māori and NZ European smoking rates is cause for particular concern. Furthermore, smoking rates are not necessarily on the decrease: rates amongst New Zealand's Pacific peoples and Asian populations (including Chinese, Korean, and Indian ethnic groups) are on the rise.

As an effort to reduce smoking rates, the NZ government funds health promotional organisations advocating tobacco control. For example, the Health Sponsorship Council (HSC) focuses on promoting smoke-free lifestyles amongst the New Zealand public, Auahi Kore focuses on promoting smoke-free lifestyles amongst Māori in particular, and the Quit group oversees the Quitline telephone support 
service and supplies health providers and individuals with print material about quitting smoking. With the exception of a blogging facility on the Quit group website, tobacco control organisations in New Zealand are currently not using persuasive technology as a channel for social marketing.

Worldwide, however, increased efforts are being made in the development of persuasive technology for smoking cessation. In the US and the United Kingdom, general purpose online community websites such as Quitnet [65] have been initiated. Often these sites position themselves almost as "one-stop shops" of facilities for people in various stages of quitting, with resources and tools that aid smokers in tracking their quitting progress. For example, several sites will generate "quitting" calendars for users, containing motivational messages on the designated quitting day, and other quitting tips and motivational messages further on in time. A related but more sophisticated website is the Australian Quit Coach (QC), which focuses on providing users with intelligently customised information, tips, and feedback. The feedback presented to users is specifically related to their current stage of quitting according to the Transtheoretical Model of general behaviour change [115]. In a different vein, quitting blogs have become a firmly established phenomenon, many of which are hosted by national-level health organisations. For example, the Quit Group hosts a blogging facility [116]. As discussed in $\S 2.1$, STOMP project researchers have used cellphones as a persuasive technology, and designed different sets of intervention messages for NZ European and Māori audiences [28].

New Zealand culture In the 2006 census, 67.6\% of the population identified as NZ European, $14.6 \%$ identified as Māori, 9.2\% identified as belonging to an Asian ethnic group, and $6.9 \%$ identified as belonging to the Pacific peoples ethnic group $^{1}$ [135]. When Hofstede profiled New Zealand in the late 1970s, he ranked it as the fifth most individualist nation out of 53 nations. Since the 1970s however, the face of the New Zealand public has changed. In particular, the proportion of the population identifying as NZ European has comparatively decreased, while the proportion of people identifying with the Māori, and Pacific peoples ethnic groups has increased [135].

Bearing in mind that Hofstede focuses on national cultures, his characterisation of New Zealand as individualist was more an indication of the minority status of Māori at the time of his investigation, rather than of Māori values. In writing about Māori values from a NZ European perspective, John Patterson states

\footnotetext{
${ }^{1}$ The ethnicity terminology we use throughout this thesis matches that used by Statistics New Zealand [135].
} 
that "Māori values tend to be radically unlike Pakeha² values [109]", and that a common fallacy of NZ Europeans is that "Deep down, Māori and Pakeha are very similar [109]". The Māori view of the self is collectivistic, as individuals are identified with their kinship groups, to the extent that the personal pronoun ' $\mathrm{I}$ ' (au) refers either to the individual or the tribe [109]. Māori traditionally take the view that the community to which one belongs makes an individual who he or she is [110]. The individual is not of overriding importance, and the concept of collective responsibility is dominant. Elders are respected and viewed as figures of authority [151]. Māori society is not egalitarian, but each person in a group has his or her own importance and is respected as part of a harmonious interlocking whole [109]. Patterson suggests respect as a fundamental Māori value, linking it to mauri, or life force, which is contained in both living and non-living objects. Mana is another key concept of Māori culture [109, 110, 151]. It is difficult to translate into English, but has been described as power, authority, standing, and has both a spiritual and ethical dimension. It is a relational concept, and expresses an active fellowship [109, 110, 151]. Mana is foremostly collective rather than individual, the mana of a family member extends to all other family members, and maintaining communal mana is considered extremely virtuous [110].

The characteristics of collective identity, collective responsibility, and respect for elders are in keeping with traits of cultural dimensional views of collectivism. Additionally, mana seems to map onto concepts of collective image, and Asian concepts of face [59], especially because building and maintaining mana are of paramount importance for group identity. All of these cultural aspects indicate that Māori culture can be characterised as strongly collectivist.

PT game domain A number of factors led to our decision to develop a game about smoking cessation. These factors included the high rates of smoking amongst the NZ public, the lack of existing culturally-relevant PT games on smoking cessation, and the characterisation of NZ Europeans as individualist, and Māori as collectivist. We decided to develop the game in two separate versions to suit the motivations, expectations, and cultural values of NZ European players and Māori players. Both versions were to be named Smoke?

From a behaviour and attitude change perspective, it is important to note here that the cessation process typically takes a number of weeks, and sometimes, years. Our time constraints led us to limit our project by focusing only on the short term persuasive effects of our games on players, as opposed to long term effects.

\footnotetext{
${ }^{2}$ The term pakeha refers to New Zealanders of European origin.
} 


\subsubsection{Process and methods}

The Prototype Development phase involved the use of a range of methods, spanning the domains of general qualitative research, $\mathrm{HCI}$, and software engineering. We discuss here how these methods played a part in the prototype development, as well as other aspects of the development process.

\subsubsection{Content analysis}

Overview See $§ 3.2 .2 .2$ for an overview of this technique.

Content analysis of smoking cessation materials To obtain relevant material for the games, it was necessary for us to learn about smoking cessation, and the steps typically recommended by cessation experts. We gathered material about smoking, smoking cessation, addiction, and behaviour change theory, from a variety of sources, including medical and psychology literature, smoking cessation websites, quitting guidelines produced by both New Zealand and international government organisations, and popular self-help titles. From this collection of resources, we established a set of common quitting tips and suggestions that we felt might be of value to a contemplative quitter. This information served as the body of learning content for the game prototypes.

\subsubsection{Focus groups}

Overview Focus groups are a qualitative method of eliciting information from a group of participants. Joe Langford and Deana McDonagh define the focus group concept as "a carefully planned discussion, designed to obtain the perceptions of the group members on a defined area of research" [89]. They are useful for obtaining background knowledge for a new project, guiding directions for future research, evaluating and obtaining insight into related research, gaining impressions of existing or proposed services, and stimulating new ideas or concepts. Typically, a focus group will comprise five to twelve participants of a demographic related to the topic of investigation. For example, if the topic under investigation is experiences of healthcare workers in nursing homes, researchers are likely to conduct focus groups with healthcare workers currently employed by nursing homes. Focus groups also require a discussion leader to guide the session. The role of the discussion leader is both to encourage the group members to contribute, and also to keep the discussion on topic. Potential disadvantages include group members feeling too shy to contribute, and the emergence of groupspeak, where all group participants claim to agree with one another. 
Focus groups with smokers and non-smokers An important feature of both game versions was their relevance to our target NZ audiences: NZ Europeans and Māori. While there is no shortage of literature surrounding attitudes and beliefs about smoking, most of it originates from the United States and Europe. To investigate local perceptions of smoking, we carried out two separate focus groups with people between the ages of 18 and 30 who self-identified as NZ European, and Māori, respectively. Any research involving Māori participants conducted at Victoria University of Wellington must be approved by the Toihuarewa, the university's Māori academic forum. Accordingly, the Māori pro-vice chancellor's opinions were sought on the rough plans for the focus group discussion, and we took note of his suggestions for cultural appropriateness and sensitivity. The focus group agenda is contained in appendix $\mathrm{F}$.

As well as incorporating a local perspective to the game, we wanted to feature perspectives of both smokers and non-smokers. Our focus group participants were a mixture of smokers, ex-smokers, and non-smokers. Each session lasted 90 minutes, and the conversation took the form of a guided discussion examining the topics of smoking, perception of smoking, smoking cessation, cultural and societal attitudes towards smoking, marketing, and social marketing. After the sessions, the recorded discussion material was analysed by way of an iterative process of code allocation and code grouping. In this way, we identified a number of categories describing the patterns that seemed most strongly present within the data from each discussion. We discuss these themes in $\S 6.1$.

\subsubsection{Semi-structured interviews}

Overview See $\S 3.2 .2 .1$ for an overview of this technique.

Interviews with cessation experts Another important perspective to include in the game was that of cessation experts, policy designers, and social workers. Using a very rough conceptual description of the game as a discussion basis, we used semi-structured interviews to determine whether the implicit advice we planned to communicate in the game was truthful, practical, and realistic. These interviews served as a preliminary form of verification for our conceptual design, and the interview responses helped us to clarify further what to emphasise during the gameplay. As well as verification, the interview material provided us with anecdotal experiences which we then worked into the existing game narrative. 


\subsubsection{Applying the input to the game design}

Overview As a fundamental objective of our study was to completely develop a functional culturally-relevant persuasive technology, informed by our culturallyrelevant PT design strategies, a principal question directing this phase of our research concerned which of our strategies to use in the different game versions and how to feature them.

The input of focus group members served as the key criterion for which of our strategies we implemented within the game. We chose only strategies that supported focus group insights, in order for the game design to reflect real culturalrelevance, as well as a New Zealand perspective on smoking cessation.

The secondary criterion was for the strategies to be compatible with and integrate with the game design. For some of the strategies in particular, it was reasonably straightforward to envisage ways in which we could embody them in the game design in a useful way, to support the persuasive objectives of the game, and also a meaningful way, to emphasise culturally-relevant persuasion. We discuss the application process of the strategies in greater depth in $§ 6.3 .1$.

\subsubsection{Reflective journal}

Overview The "Reflective Practitioner (RP)" perspective was popularised by Donald Schon in 1984 [127]. Schon argued that professionals need to move beyond a rational model of understanding, and to incorporate reflection as a work practice to challenge their own assumptions and actions. Related to the idea of RP is the reflective journal, which has been used for some time within the humanities, but is now being promoted as a useful tool in computer science as well [54]. In reflective journals, people reflect on, and critically evaluate newly encountered knowledge, connecting it with existing knowledge. This type of thinking has been shown to be conducive to future learning, as well as aiding planning and evaluation [22].

Reflective design journal of the game development process Throughout the duration of game design and development, we kept a paper design journal. In the journal, we recorded functional and non-functional requirements, brainstorming diagrams, design ideas, notes on informal interviews with experts, and high-level software designs. Given that the design and development phase lasted approximately 10 months and spanned two development iterations, we adopted a "Reflective Practitioner (RP)" perspective in keeping the journal. The RP perspective was a useful one to adopt for our project, as our design ideas were largely based on theory that had not been previously applied in PT design. It was useful to 
examine how our beliefs surrounding effective PT design changed as our project progressed, as experience proved that the realities of PT use are more ambiguous than the underlying theory on which they are based. We refer to our design journal in chapters 5 and 7 .

\subsubsection{Game development}

Overview We decided to use Macromedia Flash [92] as a development platform for Smoke? (see §5.1.1 for a more detailed discussion of the usage of Flash). The development methodology we used was an iterative one, involving two development cycles. On completion of the first iteration, we conducted a series of play tests with potential players and usability experts to assess how well our prototype met our design requirements. The findings of these tests informed how we approached the final iteration of development.

\subsubsection{Play testing}

Overview It was important for us to obtain an early picture of how players were responding to the game. The methods we used for play testing included interviews (discussed in \$3.2.2.1), the think-aloud protocol, and the cognitive walkthrough method. We overview the cognitive walkthrough method in this section, and the think-aloud protocol in the upcoming \$3.4.2.3, as it played a more significant role in the Evaluation phase of our investigation than in prototype development.

User evaluation of the initial game prototype As part of the play testing, we carried out the think-aloud protocol on the initial iteration prototype games. The evaluators consisted of a diverse group of people, including high school and university students, smoking cessation advisors, and experts. Given that a key aspect of our project was cultural relevance, it was important for us to get expert feedback on aspects of Māori culture represented in the NZM version of Smoke? So, additionally, we sent out an open invitation to representatives of the Toihuarewa, Victoria University's academic forum for Māori, asking for evaluators in a primarily advisory role. We conducted semi-structured interviews with many of the evaluators after play sessions. An analysis of the evaluation findings highlighted issues within the game design that we had to reconsider and adapt, which shaped how we approached the final phase of game development. See $\S 7.2$ for a discussion of this stage of evaluation. 


\subsubsection{Cognitive walkthrough}

Overview The cognitive walkthrough is a method often employed in usability testing in which a group of evaluators try to determine the usability of a system. The evaluator group consists of developers and usability experts, but not users. The process requires evaluators to attempt to complete a particular task by stepping through a predetermined sequence of system actions [153]. The objective is to determine whether the system is easy to learn, whether learning through exploration is supported by the system, whether the system provides enough cues to the user about actions and tasks, and, more generally, to explore overall system usability. At each step of the task, evaluators ask each other:

- Will users be able to produce whatever effect the action has?

- Will users be able to notice that the correct action is available?

- Once users find the correct action at the interface, will they know that it is the right one for the effect they are trying to produce?

- After the action is taken, will users understand the feedback they get?

Based on answers to these questions, the evaluators construct credible success or failure stories about how users might use the system, and why, with reference to their background knowledge and goals [153].

Cognitive walkthrough of the initial prototype As the second part of the play testing, we conducted cognitive walkthroughs on the game to identify the usability issues that were present in the game interfaces. The walkthrough team of evaluators consisted of three usability experts, specialising in software engineering, HCI, and video games, and the game developer. The findings yielded by the walkthrough focused our attention on major problem areas of the interface. We were then able to address them in the remaining iteration of development. See $\S 7.3$ for a discussion of the cognitive walkthrough findings.

\subsection{Evaluation}

\subsubsection{Objectives}

The two versions of the Smoke? game we developed during the previous phase of our research were based both on qualitative research conducted in New Zealand about New Zealanders' perceptions of smoking, and a subset of our culturallyrelevant strategies detailed in $\S 4.3$, selected because they supported the focus 
group findings. In evaluating the game, we saw the opportunity to validate both our research hypothesis, and more specifically, several of our strategies that informed the game designs.

In terms of explicit outcomes, our objective was to determine whether individualist players would find the version of Smoke? designed for NZ Europeans (the NZE version) more persuasive than the version designed for Māori (the NZM version), and whether collectivist players would find the NZM version more persuasive than the NZE version. Given the small scope of our study, we decided to focus only on players' short term attitude changes resulting from the game, as well as their experiences of the game. Additionally, we were interested in people's acceptance of how we had applied the strategies in the design of the two versions of the game, and their perceptions of, and reflections on our design intentions.

\subsubsection{Process and methods}

Despite the growing interest in the serious games movement, researchers have not yet agreed on a singular method to measure the persuasive effects of games. Accordingly, we decided to adopt methods used both in cross-cultural psychology to measure attitude change, and $\mathrm{HCI}$, to investigate user experience. As such, we conducted a large-scale quantitative evaluation of the effects of the two versions of Smoke? on individualist and collectivist audiences, as well as a smallscale qualitative evaluation of people's thoughts and experiences while playing the game, and their immediate reflections on it.

\subsubsection{Factorial design experiment}

Overview Factorial design experiments are commonly used in psychology studies to investigate the relationship between factors, and to establish interactions between them. The number of factors relates to the number of conditions being tested, for example, in a $2 \times 2$ design experiment, there are four conditions under investigation.

\section{Factorial design experiment of attitude changes resulting from playing Smoke?}

To obtain an overview of people's attitude changes according to the game version they played, we conducted a 2 (individualistic value orientation vs. collectivistic orientation ) $\times 2$ (NZE version $\times$ NZM version) experiment with 141 players between the ages of 17 and 25 . Of these 141 participants, 71 self-identified their ethnicity as NZ European, and 46 self-identified with the Māori or Pacific peoples ethnic group. As we discussed earlier, NZ Europeans are likely to be indi- 
vidualistic in their outlook, while Māori are more collectivistic. The Pacific peoples category is a heterogeneous group, including Samoans, Tongans, Niueans, and Cook Islanders [135]. Pacific cultures are also classified as collectivistic, in that they prioritise the values of obedience, respect, conformity, and acting in accordance with parents' wishes [126, 150]. Excessive independence is thought of as leading to the growth of negative qualities [126]. The remainder of the participants identified a range of ethnicities, including Chinese and Indian. China and India are generally regarded as collectivist cultures $[69,142]$.

In terms of smoking behaviour, 43 of the participants described themselves as current smokers, 32 described themselves as ex-smokers, and the remainder described themselves as non-smokers.

It was unexpectedly difficult to obtain Māori participants for our study. The percentage of the population identifying as Māori is small to begin with, but additionally, the numbers of Māori pursuing tertiary education are proportionally far smaller than their NZ European counterparts. One of our avenues for obtaining participants was through flyers posted around campus, but response rates from Māori individuals were quite low. To gain access to certain groups, it is sometimes necessary to involve gatekeepers, individuals who are trusted by the group members and serve as the group's interface to the wider community. In New Zealand, this is often the case for Māori and Pacific communities, particularly when the access is sought for research purposes. Consequently, we engaged in a process of contacting, and meeting with gatekeepers of final year Māori and Pacific secondary school student groups. Additionally, we had people acting as liaisons with the gatekeepers, who ensured that they introduced us to the participants before we discussed the research with them. Without these layers of social interaction, we would not have obtained the Māori player sample.

See $\S 8.1 .1$ for more details on our quantitative study.

\subsubsection{Surveys}

Overview Surveys are a standard tool for data collection in qualitative and quantitative investigation. In more quantitative roles, they are typically used to sample some portion of the population in order to be able to make generalised inferences about the population at large [35]. In experimental design, they are a commonly used tool for determining effects before and after exposure to a stimulus.

Pre- and post-surveys of the Smoke? experiment To find out whether the game changed people's attitudes towards smoking, each participant filled out a presurvey before completing one play-through of a randomly allocated version of 
Smoke? and a post-survey. Both the pre- and post-survey contained items investigating participants' general attitudes towards smoking, their intentions to quit (if they were smokers), and their resistance to smoking (if they were non-smokers). In addition, the pre-survey contained items concerning values, and the role smoking played in the participants' lives. Many of the items contained in our surveys were drawn from previous survey tools used in smoking cessation studies. See $\S 8.1 .1 .3$ for more details about the survey measures and appendix I for the complete survey.

After the survey data was collected, we analysed the data using several statistical methods, including paired sample t-tests, multi-dimensional scaling, and regression. See $\S 8.1 .2$ for an in-depth discussion of our analysis and results.

\subsubsection{The think-aloud protocol}

Overview The think-aloud protocol, sometimes referred to as the "concurrent think-aloud procedure" is a common method employed in usability testing to obtain insight into user experience [105]. The method consists of the user verbalising their thought processes while performing certain tasks on a particular application. They specifically focus on talking about their actions, perceptions, and expectations regarding the application's interface and functionality. Alongside the user, an administrator identifies and analyses where the user's task flow deviates from the ideal flow. The think-aloud protocol is especially effective in helping administrators identify usability issues in real-time and in a reasonably unobstructed context. At the same time, the administrator must try and keep the user verbalising their thoughts. The process of complete verbalisation during task completion is generally uncommon and indeed the very process of verbalisation sometimes makes users examine task performance and completion more than they would otherwise. In describing what they are doing and thinking, users become aware of the possibility of appearing inconsistent in their thoughts and actions, and sometimes begin excluding details.

Performing think-aloud with game players The items included in the surveys for the quantitative factorial design experiment were selected with statistical analysis in mind. We were concerned that they were not finely-tuned enough to highlight less easily observable game responses, and in particular, the chains of reasoning players used in their responses. We decided to include a qualitative form of evaluation to tease out further subtleties of people's preferences relative to the game versions. We were especially keen to obtain some insight into people's opinions of the game versions while they were playing, so we decided to use the 


\begin{tabular}{|l|l|}
\hline \multicolumn{2}{|c|}{ Description of players of the NZE version of Smoke? } \\
\hline \hline Code name & Description \\
\hline E1 & NZ European female smoker in her early 20s \\
\hline E2 & NZ European female non-smoker in her early 20s \\
\hline M1 & Māori female smoker in her early 20s \\
\hline M2 & Māori male non-smoker in his mid 20s \\
\hline
\end{tabular}

Table 3.1: Players of the NZE version

\begin{tabular}{|l|l|}
\hline \multicolumn{2}{|c|}{ Description of players of the NZM version of Smoke? } \\
\hline \hline Code name & Description \\
\hline E3 & NZ European male non-smoker in his mid 20s \\
\hline E4 & NZ European male non-smoker in his early 30s \\
\hline M3 & Māori male non-smoker in his mid 20s \\
\hline M4 & Māori female smoker in her mid 20s \\
\hline
\end{tabular}

Table 3.2: Players of the NZM version

think-aloud protocol.

There were eight player participants in total, four of whom were of NZ European descent, and four of whom were of Māori descent. Two NZ European and two Māori players were assigned to play the NZE version of Smoke?, while the remaining two NZ European and Māori players were assigned to play the NZM version. We summarise the details of the players in Tables 3.1 and 3.2.

The players were given brief summaries of the think-aloud protocol, and then asked to complete one run-through of the game. Four graduate level, crosscultural psychology students played the role of administrator, with each student conducting sessions with two of the eight players. To aid the administrators in later analysis, we audio and video recorded the think-aloud sessions. Afterwards, the administrators reviewed their session data, and presented us with their key observations. We discuss the findings of the think-aloud sessions in $\S 8.2 .2$ and $\S 8.2 .3$.

\subsection{Delimitations, limitations, and other study con- siderations}

Perspective on culture and cultural dimensions The theory-driven stages of this work, including the examination of Fogg's strategies, and the discussion of culturally-relevant PT strategies, reference general concepts of individualism and collectivism, and associated cross-cultural psychology literature. We view individualism and collectivism as two ends of a bipolar continuum, as opposed to 
two distinct but related unipolar dimensions.

Cultural classification Where this research calls for categorisations of people's culture and values, we use a shortened version of Schwartz's values survey. While it has not been widely used within $\mathrm{HCI}$, it is frequently used in psychology research at the individual level, whereas the research of Hofstede does not apply at this level. As discussed in §2.2.2.4, Schwartz's dimensions can be differentiated from most other proposed sets of dimensions, because they were a priori theorised, and are strongly supported by empirical validation.

Domains informing our theory During the theory development phase of this study, we interviewed stakeholders in organisations falling under the umbrella of social marketing, promoting financial literacy and tobacco control. We did not focus on commercial organisations.

Form of our PT We decided to use games as a platform for our culturallyrelevant persuasive technology. This impacted on both how we were able to apply our design strategies, and also the player evaluations of our culturallyrelevant persuasive technology.

Domain of our PT In accord with our focus on social marketing, we chose smoking cessation as the topic of our culturally-relevant persuasive technology. Smoking is a widespread problem in NZ, and affects both the NZ European population and the Māori population.

Target audiences of our PT game Our PT prototype is firmly within the New Zealand context, designed for NZ Europeans and Māori, and tested by NZ European, Māori, and Pacific peoples participants. This makes our evaluation of the prototype necessarily limited in terms of its generalisability.

Demographics Variables such as age, gender, and socio-economic background help shape people's attitudes and preferences. Given the short time-frame for this research, however, we decided to not control these variables. During the development and evaluation phases of this study, we analysed data according to research participants' self-identified cultural affiliations and values.

Time span of evaluation Smoke? was designed to be played more than once. It contains a number of narratives, illustrating the consequences of adopting different attitudes towards the goal of cessation, and presenting different smoking ces- 
sation information. Each participant involved in its evaluation, however, played it through just once, and provided responses for it immediately after playing. As such, their responses reflect attitudes and intentions, as opposed to behavioural changes. Additionally, their responses reflect a short-term, single-exposure to the game. We expect that repeated exposure to the game may have yielded stronger attitude change effects in the participants.

Ethical considerations It is required at Victoria University of Wellington that any research involving human participants must be granted approval from the Human Ethics Committee before it can proceed. For all parts of this research requiring human input, consent was sought in advance. Furthermore, participants were all supplied with clear information about the tasks they were to perform, how it would aid our research, how they could access publications relating to the research, a date by which they could withdraw their participation, and consent forms. Although we were not able to treat participants as anonymous, the information they supplied was treated as confidential, and, where necessary, identities were concealed. See appendices A, B, C, D, E, F, G, H, and I for the documents supplied to the participants.

In addition, as part of the University's obligation towards the Treaty of Waitangi, research involving Māori, or Māori issues, must be discussed and approved by the Toihuarewa, the Māori academic forum. Throughout this project, ongoing communication was maintained with Te Toiahurei, the Māori pro-vice chancellor, as well as various other Toihuarewa members. They supplied us with useful feedback and insight regarding sensitivity to Māori cultural issues. 


\section{Chapter 4}

\section{Culturally-Relevant PT Strategies}

Persuasion is about changing people's attitudes or behaviours. To design persuasive technologies that are most meaningful and effective for their target audiences, we claim that designers should reference the audience's cultures in their design. In this chapter, we summarise the findings of interviews we carried out with stakeholders of social marketing organisations in New Zealand, to establish how they incorporate culture as a factor in their current persuasive approaches. We then examine the motivations behind each of Fogg's PT tool strategies, in the context of the cultural dimensions of individualism and collectivism. Finally, using insights from the interviews, our examination of Fogg's strategies, as well as the cross-cultural psychology literature on behavioural tendencies of individualists and collectivists, we present our first research contribution: a set of culturallyrelevant persuasive technology design strategies.

\subsection{Social marketing stakeholder interviews}

Social marketing has been defined as "use of marketing principles and techniques to influence a target audience to voluntarily accept, reject, modify or abandon a behaviour for the benefit of individuals, groups, or society as a whole" [87]. Social marketing focuses on target audiences, so societal demographics should play an important factor in determining communication approaches.

To investigate whether current NZ social marketing approaches reflect the country's increasingly multicultural population, we conducted interviews with stakeholders of two well-known social marketing organisations. We interviewed four members in each organisation filling roles in policy design and evaluation, brand communication, or upper management. One of the organisations, referred to as "Future", is primarily concerned with educating people about financial matters and retirement planning. The other organisation, "Support", focuses on re- 
ducing smoking rates in the Māori population which demonstrate the highest smoking rates of the main ethnic groups in NZ [12]. In §3.2.2.1, we discussed how we analysed the interviews to identify three overall themes. We discuss these themes next, supporting each with extracts from the interviews, and crosscultural theory-based discussion.

\subsubsection{Universal relevance: the views of NZ European stakehold- ers}

Discussions with NZ European staff from Support and Future showed that, while they were aware that context is important in grounding a persuasive appeal, there was an underlying belief in the universal relevance of their messages:

... the messages we want to get across are the same for everybody but the mechanisms by which we want to get the messages across may differ... the content, the messages, everything's the same, the same mathematical principles apply. - Executive Director of Future

Additionally, the NZ European staff members of Future were hesitant about developing culturally-relevant versions of campaigns, because they considered the within-culture diversity to be too great to accommodate:

We're not going to treat [people of one] culture as one single entity that all think and act the same way. Within any culture there's such a variety of views and behaviours that it would be quite wrong to say, "Right, that's how Māori think", because there is a variety of views and approaches to life in Māori culture as there is in any other culture.

- Executive Director of Future

They considered that customisation was the best way to deal with diversity:

We acknowledge that people are very different. It's not about saying "you should be saving for retirement" or "you should be doing this" or "you should be doing that", it's about providing education and information so people can make their own decisions. You're the one provided you've got all the information that then chooses to save or not to save or to pay off more debt or pay off less debt depending on what's really important to you.

- Marketing communications manager of Future 
The extract emphasises that the Future approach was focused on personal customisation and individual empowerment, but relied heavily on personal responsibility. This approach has significant implications because while personal responsibility is nurtured and encouraged in members of individualist cultures, it is generally less actively emphasised in members of collectivist cultures $[69,96,142]$.

\subsubsection{Reduction of relevance: the views of Māori stakeholders}

A Māori staff member of Support expressed his concern about the effectiveness of communication focused on individual empowerment on Māori audiences:

...there's a real risk of coming in at a national level and focusing on individuals and not really thinking about what we're trying to do. You can think about a person's behaviour in terms of the individual behaviour but that's also often influenced by their family and peer environment and sometimes the community environment.

- Senior researcher of Support

Family and community influence play a stronger role in collectivist cultures than in individualist ones [38, 69, 142].

One of the interviewees was a cultural auditor of Māori descent, who had been contracted by Future to identify how successfully it was reaching Māori and Pacific audiences. Agreeing that mainstream NZ social marketing approaches were not suited to collectivist cultures, he noted about Future's communication strategy:

...we found little evidence of inclusiveness of language, of concepts, of media preference or attempts to understand the Māori and Pacific mindsets in relation to [Future]...most of what [Future] communicates registers financial issues from an ethnocentric perspective... which in turn alienates some Māori and Pacific audiences.

- Cultural auditor of Future

He went on to state that the communication gap with Māori and Pacific audiences may have arisen because the development of culturally-relevant communication was not defined as an objective:

...[Future] does not define communication audiences by ethnicity...it doesn't have any objectives to reach Māori and Pacific key audiences...the creative material therefore wasn't created to engage with Māori or Pacific people per se but generally it attempts to assimilate Māori and Pacific people as part of 
mainstream NZ...when you take a mainstream idea and thought and try and force it, it's like putting a square peg into a round hole, it just doesn't work. - Cultural auditor of Future

The Future cultural auditor did, however, believe that there was a way to develop more culturally-relevant persuasive appeals:

...tap into the cultural capital and those values and mindsets, and you can be well on your way to connecting a lot stronger.

- Cultural auditor of Future

\subsubsection{A collective focus: the views of Māori stakeholders}

A recurring theme that emerged from discussions about how best to persuasively communicate with Māori and Pacific audiences was the leveraging of existing social networks, including family, communities, and tribal groupings. One Support member suggested that campaigns should include:

... a facilitation process where the whole community comes together and operates in a bigger way and identifies the issues that they see important and comes up with solutions and they engage in making changes.

- Senior researcher of Support

While community support is likely to be appreciated by people of all cultures, the Future cultural auditor explained why collective motivations are more effective than personal motivations with Māori and Pacific audiences:

...they have a collective responsibility towards issues...their priorities are collective responsibilities rather than "I've gotta look after myself at all costs"...this means that service to others is more acceptable than looking after yourself first, service is thought of both as a burden and as an honour.

- Cultural auditor of Future

Interestingly, the question of whether collective interests are prioritised over personal interests is one of the most fundamental identifiers of whether cultures are collectivist or individualist [142].

Another significant theme that surfaced from interviews with Māori stakeholders was how effective communication with Māori and Pacific audiences was linked to whether they felt that they had ownership of the campaign messages. The Future cultural auditor commented about a NZ transport safety social marketing campaign, which he believed was an example of successful communication with Māori and Pacific audiences: 
...get the community to work with you...what's developed from it is that the community started to own the messages, started to take responsibility and started to enforce some drink drive issues, or road safety issues and that's an example of how the process can change, still doing the same thing, but just changing the process.

- Cultural auditor of Future

Support focuses on Māori audiences, and uses existing family and community links to deliver its persuasive communication:

When you think about Māori, it's about whanau', about family, it's about that greater family, it's about iwi ${ }^{2}$, it's about your hapu ${ }^{3}$, it's about all these people working together so that's how we work to support each other for the greater good of the people...so we support the community which then supports the health worker in their relationship with that person in the community or that marae 4 , so it's not about "me", it's about that community base working. - Campaign manager of Support

\section{Discussion}

The interview findings illustrate that between the NZ European and Māori social marketing stakeholders, there was an appreciable difference in how the effectiveness of current social marketing persuasive approaches being used in New Zealand was perceived.

The NZ European participants believed that individual empowerment was the most effective persuasion approach. They also felt that personal customisation was the best solution for dealing with diversity.

In contrast, the Māori participants argued that Māori and Pacific audiences should not be approached in the same manner as the mainstream, NZ European audience. They suggested that Māori and Pacific audiences would respond more positively to references to collective values and social structures.

In general, the NZ European participants believed that cultural context was not an issue of major concern. This view is reminiscent of Hall's views of the "cultural unconscious" (see §3.1.1), where people embedded in a certain culture are unaware of its characteristics.

\footnotetext{
${ }^{1}$ Whanau as a concept loosely corresponds to "family", however it can be inclusive of distant relatives and friends.

${ }^{2}$ Iwi roughly corresponds to "tribe", "clan", and sometimes "backbone".

${ }^{3} \mathrm{Hapu}$ refers to a social grouping smaller than iwi, so it may be conceptualised as a "sub-tribe".

${ }^{4}$ Marae is the sacred area of land in front of a traditional meeting house.
} 


\subsection{Fogg's PT strategies from a context of individual- ism and collectivism}

In $§ 2.1 .1$, we overviewed Fogg's PT tool strategies, a set of techniques commonly used in persuasive technology tools to make people's desired outcomes easier to achieve. Here we revisit Fogg's PT tool strategies, but this time we examine them by focusing on their underlying motivations.

As discussed in $\S 2.2 .3$, individualism and collectivism represent the most wellresearched cultural dimensions to date, and they have been widely used in crosscultural psychology studies of persuasion. Therefore, in our work we have decided to concentrate on these dimensions. Referring to the cross-cultural psychology literature on individualism and collectivism, we match the motivations underlying Fogg's PT tool strategies with typical motivations of individualists and collectivists. In table 4.1, we briefly explain the motivations that our discussion may refer to.

\begin{tabular}{|l|l|}
\hline \multicolumn{2}{|c|}{ Individualist motivations referenced in strategy discussion } \\
\hline Independence & Society members are accustomed to independence. \\
\hline Selfidentity & Society members have a primarily individual identity. \\
\hline Consistency & Society members value consistency. \\
\hline Few rules & Society has few rules. \\
\hline Rewards & Society favours use of positive reinforcement. \\
\hline Competition & Society members are accustomed to being competitive. \\
\hline \hline \multicolumn{2}{|c|}{ Collectivist motivations referenced in strategy discussion } \\
\hline Interdependence & Society members are accustomed to interdependence. \\
\hline Group identity & Society members are very influenced by their group identity. \\
\hline $\begin{array}{l}\text { Situational } \\
\text { relevance }\end{array}$ & Society members prioritise context over consistency. \\
\hline Many rules & Society has many rules. \\
\hline Punishment & Society condones use of punishment. \\
\hline Face & Society members are concerned with upholding face. \\
\hline Conformity & $\begin{array}{l}\text { Society members are concerned with conforming to ingroup ex- } \\
\text { pectations. }\end{array}$ \\
\hline Harmony & $\begin{array}{l}\text { Society members value harmony amongst members of their in- } \\
\text { group. }\end{array}$ \\
\hline
\end{tabular}

Table 4.1: Individualist and collectivist motivations

Our discussion of each of Fogg's strategies is presented in a standard format, consisting of Description: an explanation of the strategy; Antecedents and consequences: a cross-cultural psychology literature-based discussion of factors leading to, and resolved by, use of the strategy; Summary of key motivations: the important individualistic and/or collectivistic motivations referenced in discussion; Target 
audience: whether the strategy is better suited to individualist or collectivist audiences; and Related strategies: strategies with similar motivations, and ones with opposed motivations ${ }^{5}$. The REDUCTION and TUNNELLING strategies have been discussed together, as they have similar underlying cultural motivations.

\subsubsection{The REDUCTION and TUNNELLING strategies}

Description The REDUCTION strategy concerns making target behaviours easier by reducing a complex activity to a few simple steps. The TUNNELLING strategy also reduces complexity and leads users through a predetermined sequence of actions or events, step by step, to encourage certain behaviours.

Antecedents and consequences Fogg states that the effectiveness of these strategies is partly explained by self-efficacy, defined as "belief in ones capabilities to organise and execute the courses of action required to produce given attainments" [15]. The reduction of the complexity of action increases people's self-efficacy. In terms of the social psychology theory of planned behaviour (TPB) [8], an increase in self-efficacy related to a certain behaviour corresponds to an increase in perceived control over performance of the behaviour. People of individualist cultures are more likely to be concerned with self-efficacy than people of collectivist cultures. In a recent study investigating the role of self-efficacy in individualists and collectivists, self-efficacy was found to be more relevant as a predictor of performance for individualist, rather than collectivist participants [84]. This is likely connected to the important role of independence in individualist cultures $[69,142,143]$. Individualists are, therefore, more likely to attempt to solve problems on their own, rather than rely on others for help. We observe that the TPB, which provides one explanation of the utility of REDUCTION and TUNNELLING, is a Western psychological theory. It concerns people's beliefs about their abilities to act from an autonomous perspective, and therefore is based on an individualistic worldview.

Another perspective in analysing the effectiveness of REDUCTION and TUNNELLING is to look at what they aim to minimise, namely, inconsistency, and complexity of problem solving and decision making. It is widely accepted that in individualist cultures, people are more concerned with attitude-behaviour consistency, and consistency of action, than people of collectivist cultures, where decisions and actions are situationally determined [69, 96, 142, 143].

\footnotetext{
${ }^{5}$ These sections may reference the following strategies, which we introduce in $\S 4.3$ : GROUP CUSTOMISATION, HARMONY, GROUP OPINION, MENTORING, DIFFERENCE MONITORING, DISESTABLISHING, TEAM PERFORMANCE, and ACHIEVEMENT.
} 
Summary of key motivations Independence and consistency.

Target audience Members of individualist cultures.

Related strategies The underlying motivations of REDUCTION and TUNNELLING are similar to those of ACHIEVEMENT. Its motivations contrast with those of GROUP OPINION, MENTORING, and SUGGESTION. The motivations of REDUCTION and TUNNELLING are both similar to, and contrast with those of SURVEILLANCE.

\subsubsection{The TAILORING strategy}

Description The TAILORING strategy involves providing users with information relevant to them, with the objective of persuading them to change certain attitudes or behaviours.

Antecedents and consequences The process of TAILORING requires modifying application set-ups to suit particular users' needs and habits. Social psychology persuasion studies premised on dual process theories of persuasion have shown that people tend to pay more attention to information that they believe is relevant to them $[113,114]$.

A typical assumption around TAILORING is that it is personal in nature, and that the user in question is an individual, with personal needs, habits, and preferences. A noted characteristic of individualist cultures is that the basic social unit consists of the individual, and society members are socialised to be independent $[69,96,142,143]$. This makes the assumption of an individual and independent context of use more likely to appeal to individualist audiences.

Furthermore, the process of individually-oriented TAILORING requires users to have well-defined self-identities, or identities in more autonomous contexts. The notion of an autonomous and stable self-identity is characteristic of members of individualist cultures, rather than collectivist cultures $[69,142,96]$.

Summary of key motivations Independence and selfidentity.

Target audience Members of individualist cultures.

Related strategies The underlying motivations of TAILORING are similar to those of SELF MONITORING and ACHIEVEMENT. Its motivations contrast with those of 
GROUP CUSTOMISATION, MENTORING, and TEAM PERFORMANCE. The motivations of TAILORING are both similar to, and contrast with those of SURVEILLANCE.

\subsubsection{The SUGGeSTION strategy}

Description The SUGGESTION strategy is premised on the idea of intervening at a right time, as people feel more motivated to perform some behaviours at certain times over others.

Antecedents and consequences Research shows that there are specific kinds of scenarios in which people are more susceptible to influence [33,46]. Under typical conditions, however, people of individualist cultures are more likely than collectivists to look to their own values and priorities to help them determine their courses of action [38,96, 142]. In contrast, people of collectivist cultures are more accustomed to relying on the opinions and suggestions of other ingroup members, or looking to social norms to decide their actions [38, 96, 142]. They actively seek out these opinions, and are not necessarily aware that they are doing so. This indicates that collectivists are generally more reliant on suggestion than individualists, making the possible usage of persuasive technology in a SUGGESTION role less of a conceptual leap. This role can be of particular significance if the persuasive technology is viewed as a trusted ingroup member (as in the computers as team mates experiment discussed by Byron Reeves and Clifford Nass in the The Media Equation [119]).

An additional benefit is that if a suggestion comes from a persuasive technology, then no one will suffer public loss of face, which is a risk of pointing out behavioural suggestions to group members in collectivist cultures [51,59, 142].

Summary of key motivations Situational relevance and face.

Target audience Members of collectivist cultures.

Related strategies The underlying motivations of SUGGESTION are similar to those of GROUP OPINION, MENTORING, DIFFERENCE MONITORING, and SURVEILLANCE. Its motivations contrast with those of REDUCTION, TUNNELLING, and ACHIEVEMENT. 


\subsubsection{The SELF MONITORING strategy}

Description The SELF MONITORING strategy allows people to monitor themselves to modify their attitudes or behaviours to achieve a pre-determined goal or outcome.

Antecedents and consequences Essentially, SELF MONITORING technologies facilitate people learning more about themselves, and their own behaviour. Although at a fundamental level people are intrinsically curious about themselves [15], beliefs about the importance of self information differ according to cultural context [5]. Self information and knowledge is likely to be more important to individualists rather than to collectivists. The self-identities of individualists tend to be autonomously defined, whereas the self-identities of collectivists are partly defined by attributes of their ingroup $[69,96,143]$.

In addition, SELF MONITORING information is used to inform users of how close they are to achieving a personal goal. Beliefs that they have autonomous control over goal achievement are more likely to be held by individualists, rather than collectivists $[38,84,96]$.

Finally, SELF MONITORING focuses on personal goals. Personal goals are more ultimately more important than other kinds of goals in individualist cultures [142, 69].

Summary of key motivations Self identity and independence.

Target audience Members of individualist cultures.

Related strategies The underlying motivations of SELF MONITORING are similar to those of TAILORING and ACHIEVEMENT. Its motivations contrast with those of GROUP CUSTOMISATION, MENTORING, and TEAM PERFORMANCE. The motivations of SELF MONITORING are both similar to, and contrast with those of SURVEILLANCE.

\subsubsection{The SURVEILLANCE strategy}

Description The SURVEILLANCE strategy involves allowing one party to monitor the behaviour of another party for the purpose of modifying a specific behaviour, as when people know they are being watched, they behave differently [145]. 
Antecedents and consequences SURVEILLANCE technologies are often used in group settings, such as restaurant kitchens, or computer networks. An affordance of the group setting is that the context of performing a behaviour becomes somewhat situationally relevant, and partly a matter of whether the behaviour seems reasonable in relation to the behaviour of other group members. Accordingly, group norms are activated as a basis for behaviour-specific decision making. Group norms are generally more effective on people who actively identify with the group, who believe that a deviation from their norms would disrupt normal group interaction patterns and group harmony, which may lead to a loss of social approval from other group members [83]. People of collectivist cultures are more concerned with upholding the norms of their ingroup than people of individualist cultures $[69,142,143]$.

In addition, at a more fundamental level, collectivists are accustomed to what could be termed as "surveillance", both covert and overt, as a fact of life in socially dense communities $[69,142]$. While surveillance has negative connotations in Western cultures, in collectivist cultures it has acknowledged positive consequences. For example, ingroup members might observe each other's actions, and be inspired to change their own behaviour. Furthermore, an awareness of the responsibilities of the role model relationship may motivate observed parties to continue to act as positive role models, creating a two-way benefit.

At the same time, SURVEILLANCE is not only used in group settings because sometimes a behavioural comparison with others is irrelevant. In such situations, SURVEILLANCE works to foster (or deter) particular behaviours on an individual basis, and operates as a type of SELF MONITORING, except with an additional party featured in the feedback loop. As we discussed earlier, SELF MONITORING relies on individualist motivations such as independence, and self-identity.

Summary of key motivations Situational relevance, group identity, harmony, interdependence, independence, and self identity.

Target audience Members of individualist and collectivist cultures.

Related strategies The underlying motivations of SURVEILLANCE are similar to those of SUGGESTION, HARMONY, GROUP OPINION, and DIFFERENCE MONITORING. Its motivations are both similar to, and contrast with those of TAILORING, REDUCTION, TUNNELLING, SELF MONITORING, GROUP CUSTOMISATION, MENTORING, TEAM PERFORMANCE, and ACHIEVEMENT. 


\subsubsection{The CONDITIONING strategy}

Description The CONDITIONING strategy concerns the use of operant conditioning to reinforce target behaviours when they occur. When used in persuasive technology, it generally takes the form of positive reinforcement, such as rewarding the performance of target behaviour.

Antecedents and consequences In discussing CONDITIONING, Fogg adopts a strong stance against the use of technology to punish [46], reflecting desirable parenting and teaching styles of individualist cultures, along with Western ethics [46, 142]. At an individual level, people of all cultures probably enjoy positive encouragement, but at a cultural level, attitudes towards the effectiveness of positive reinforcement as a method of teaching people about rule adherence diverge $[142,126]$.

This may be a function of the number of societal rules that need to be internalised, which Triandis refers to as looseness and tightness of cultures. In loose cultures, which tend to be individualist, there are not many protocols regarding correct conduct, and considerably more latitude, whereas in tight cultures, which tend to be collectivist, there are numerous, and rigid protocols governing behaviour [142]. Loose culture society members therefore focus more on learning proper conduct, and less on learning improper conduct. Tight culture society members, in contrast, spend as much, if not more, time learning about improper conduct, alongside proper conduct. The use of the CONDITIONING strategy supports a focus on learning and rewarding proper conduct, which is more in line with teaching strategies of loose, individualist cultures.

Summary of key motivations Rewards and few rules.

Target audience Members of individualist cultures.

Related strategies The underlying motivations of CONDITIONING contrast with those of DISESTABLISHING.

\section{Discussion}

Of the seven tool strategies described by Fogg and commonly used in persuasive technology, the motivations underlying the strategies of TUNNELLING, REDUCTION, TAILORING, CONDITIONING and SELF MONITORING are more suited to individualist users than collectivist users. The motivations underlying the SURVEILLANCE strategy seem well suited to both individualist and collectivist users. Only 
the motivations of the SUGGESTION strategy are better suited to collectivist users. The findings of this examination of motivations of Fogg's PT tool strategies add weight to our earlier speculations, regarding PT tools and strategies of individualist origin reflecting a bias towards individualist cultures.

\subsection{A set of eight culturally-relevant PT design strate- gies}

The examination of Fogg's PT tool strategies presented in $\S 4.2$ leads to two insights. First, it clarifies that the strategies currently most commonly used in general purpose, persuasive technology are more suited to individualist, rather than collectivist audiences.

Second, it directs us towards the idea that with contextual modifications, it is possible to design persuasive technology to better suit collectivist audiences. Additionally, the social marketing stakeholders we interviewed (see §4.1) made useful suggestions about effective communication with collectivist target audiences.

We now present a set of eight culturally-relevant PT design strategies that we have developed from the cultural perspectives of individualism and collectivism. Seven of these strategies are aimed at use in tools developed for collectivist users, and one of them is aimed at use in tools for individualist users.

Many of the collectivist-focused strategies we have developed are designed for use in the context of ingroup goals. Consequently, they are premised on the assumption that ingroup members are supportive of the group goals, and that members welcome the sharing of personal information with other members. We suggest that this is likely to be the case for collectivist users, especially if they are aware that it will facilitate progress towards a group goal. While there are any number of circumstances that may lead to ingroup members deciding that do not want to to pursue an ingroup goal, one of the fundamental characteristics of collectivist cultures is the prioritisation of group goals over personal ones [69, 96, 142].

Each of our eight design strategies is presented in a standard format: Target audience: whether the strategy is better suited to individualist or collectivist audiences; Antecedents: literature- and interview-based motivations leading to the strategy; Description: an explanation of the strategy; Real world parallel: a manifestation of the strategy in the real world; Hypothetical PT example: how the strategy might be used in a hypothetical persuasive technology; Consequences: issues resolved by use of the strategy; Summary of key motivations: the important indi- 
vidualistic and/or collectivistic motivations referenced in discussion; and finally Related strategies: strategies with similar motivations, and ones with opposed motivations.

The presentation format of our strategies differs from the format in which we examined Fogg's strategies. We adopted the different format for our own strategies, as we specifically established them to aid designers in designing culturallyrelevant persuasive technology for certain target audiences. In particular, the separated discussion of Antecedents, Hypothetical PT example, and Consequences is intended to facilitate designers in understanding how the strategy arose, and how it may be used in a tool.

\subsubsection{The GROUP CUSTOMISATION strategy}

Target audience Members of collectivist cultures.

Antecedents As we have stated earlier, collectivists' self-identities are intrinsically linked with the identity of their ingroup [59, 69, 96, 142, 143]. This finding was echoed by our social marketing interview participants, with regard to Māori and Pacific culture. Currently, many of the persuasive technologies designed for North American audiences emphasise personal customisability [46]. The emphasis on individual settings may not be as relevant for people who primarily think of their identity as part of a group identity, and are interdependent with members of their group. This is especially true if the context of use for the application is one that people will want to use with other group members, as opposed to by themselves.

Description The GROUP CUSTOMISATION strategy involves supporting groups of users to customise application settings to suit group preferences, or to specify group-relevant characteristics during application set-up. It is similar to the TAILORING strategy, but it assumes that the fundamental social unit is the group, as opposed to the individual.

Real world parallel Sociological studies of attitudes towards domestic money across cultures show that there are significant differences in how money is treated. Among Anglo-Celtic Australians, people are likely to share money with their marital partners, but money is considered a private matter, and people are unlikely to want to discuss it with others. In Asian, African, and Polynesian cultures, on the other hand, domestic money is much more likely to be shared with the extended 
family, and once children are able to support themselves, there is often a two-way flow of money between parents and children [134].

Hypothetical PT example: group financial planner The group financial planner is a web-based tool developed for a group of people who want to share their spending records and financial goals. It aids users to record and manage their expenditures, to perform calculations on paying off mortgages and debts, and to suggest realistic payment goals based on current spending habits. In addition, it can be used to send out email and cellphone text message reminders about financial goals.

During the sign up process, users set up a group profile, in which they specify details such as combined incomes, major investments, and debts of the group members. They also agree on financial goals for the group, savings priorities and goals, dept payment plans, investment plans, and links to other related group profiles. Group members have the option of setting up individual sub-profiles, affiliated to the group profiles, if they want to track their own details as well. Later, when users log into the tool, the group profile details are initially retrieved. Any changes users make with the tool are stored against their group profile.

Consequences The GROUP CUSTOMISATION strategy shifts the context of customisation from the individual to the ingroup. This supports a collectivist perspective that the ingroup forms the basic social unit rather than the individual $[51,59,69,142,143]$. In the same way that people from individualist cultures have a good understanding of their own personal needs and preferences, people of collectivist cultures understand the needs of their ingroups [69, 96, 142, 143]. Group customisation, therefore, is likely to have more resonance with many collectivists than individual customisation, and information generated from group profile details may be more meaningful to users.

Additionally, the customisation focus on ingroup identity helps foster a sense of message ownership. By framing desired outcomes in terms of the ingroup, the persuasive aims are likely to be perceived as more supportive of the group's own goals. Furthermore, once the group customisation has been set up once, other ingroup members can readily join, saving time and energy.

An important aspect of group customisation is that it can also foster ingroup face amongst members. By co-operating and collaborating with other ingroup members to achieve desired shared goals, users can feel they are playing a part in upholding collective responsibility and ingroup reputation.

Summary of key motivations Group identity, interdependence, and face. 
Related strategies The underlying motivations of GROUP CUSTOMISATION are similar to those of HARMONY, GROUP OPINION, TEAM PERFORMANCE, MENTORING, and DIFFERENCE MONITORING. Its motivations contrast with those of TAILORING, SELF MONITORING, and ACHIEVEMENT. The motivations of GROUP CUSTOMISATION are also both similar to, and contrast with motivations of SURVEILLANCE.

\subsubsection{The HARMONY strategy}

Target audience Members of collectivist cultures.

Antecedents Collectivists often choose to live in close proximity with their ingroup members and often, three generations of a family cohabit the same dwellings $[109,142,162]$. From an early age, socialisation in collectivist cultures emphasises interdependence, and the importance of acting well to preserve harmony and facilitate co-operation $[51,59,69,96,142,162]$. The drive to retain a harmonious atmosphere even impacts on typical approaches to conflict resolution. Collectivists will avoid confronting problems directly, unless it is absolutely necessary $[51,59,96,162]$. Related to the preservation of harmony is the drive to act in the best interests of the group. Both our interview data and the cross-cultural literature support the notion that collectivists give precedence to ingroup interests and responsibilities over personal ones [51, 59, 69, 142, 143]. Furthermore, all the Māori social marketing stakeholders we interviewed felt strongly that Māori and Pacific audiences would be more likely to benefit from, and feel persuaded by, community-supported and endorsed social marketing initiatives, rather than the individually-targeted initiatives that currently predominate in New Zealand.

Description The HARMONY strategy involves the presentation of social density cues to users. The cues serve to suggest to users that they are in a socially dense environment with members of their ingroup, in order to subtly promote harmonious actions that support the group's goals.

Real world parallel In many Japanese organisations, employees are perceived as interchangeable [99]. To maximise productivity, organisations strive to foster a sense of group identity within functional teams, and to integrate employees within their team. Activities might include collective meals, team dormitories, team banners, and team names. Many Japanese executives refer to their company members as a family [64]. 
Hypothetical PT example: supermarket item scanner The supermarket item scanner is a hand-held device, similar to those used already in some self-service contexts. The scanner includes slots for individual profile cards. When a card is inserted into the scanner, the card wirelessly communicates the user's identity to the supermarket database, and continuously uploads information about the items they are scanning. The profile card can be used in group mode, where it is associated with other card holders who together form a shopping club. Card holders might choose to form a shopping club if they pay for their shopping from a shared bank account, or if they live together and want to access information about specific shopping preferences and habits.

When used in group mode, users are greeted with a welcome message that lists other club members. This serves as a subtle reminder for users to shop with their club's interests in mind. If a club has a shared goal, their welcome message explicitly states this goal. For example, the device might list the important shared goals: "Spend less than or equal to \$300 a week", "20 treat items only".

Consequences The HARMONY strategy uses social density cues to remind users of their ingroup's goals. The cues remind users of how they can act in the group's interests, and assist in maintaining harmony within the group. Users are then motivated to make decisions and act based on supporting the group's goals as far as possible.

There are inevitably contexts in which users feel obliged to go against the wishes of the ingroup. This strategy only applies to situations in which individuals have already decided that they want to support the wishes and goals of their ingroup.

Summary of key motivations Harmony and interdependence.

Related strategies The underlying motivations of HARMONY are similar to those of GROUP CUSTOMISATION, GROUP OPINION, MENTORING, TEAM PERFORMANCE, and SURVEILLANCE. Its motivations contrast with those of ACHIEVEMENT.

\subsubsection{The GROUP OPINION strategy}

Target audience Members of collectivist cultures.

Antecedents Collectivists tend to place more importance on the opinions of others in their ingroup than do individualists when making decisions [38, 51, 69, 96, 142]. They are more likely to be concerned about acting in the best interests of 
the group over their own interests, and group interests might change according to the situation $[38,51,69,96,142]$. As a result, the opinions of others often have a strong shaping influence on their decisions [4,111]. Research has also shown that collectivists tend to be less effective if they feel they are performing an activity in isolation, while being more effective in group situations [69, 142]. The Māori social marketing stakeholders we interviewed supported this finding. They considered that social marketing campaigns would be more successful among Māori and Pacific audiences if they factored in community involvement. Accessing the opinions of other ingroup members is not always feasible, however, and can be time consuming.

Description The GROUP OPINION strategy involves providing users with the opinions of other ingroup members, or users similar to them ${ }^{6}$ at moments when users are required to make important decisions related to their own goals.

Real world parallel In a study of cross-cultural e-commerce adoption, researchers investigated buying behaviours of consumers from China and the United States. Societal norms were found to play a major role in influencing transaction intentions among Chinese consumers, while playing no appreciable role in the transaction intentions of the American consumers [111].

Hypothetical PT example: supermarket item scanner One shopping club member intends to buy multigrain bread, but on reaching the bread aisle, is confronted by many different brands. She cannot decide which bread to buy, so she uses her scanner device to refer to her shopping club's purchase history. Browsing the list, she can determine whether there are clear preference patterns among club members and make a confident and informed decision.

Consequences The group opinion strategy partially simulates the experience of making a decision by consulting other ingroup members. Being reminded of the existence of the group in a decision making context may help an isolated group member feel more supported and assist both the accuracy and speed of the decision making process.

The sense of group connection is important. Collectivists respect and place significant trust in the opinions of ingroup members, and are therefore likely to feel more confident about a decision if they have their input. If the decision is one

\footnotetext{
${ }^{6}$ The similarity might be based on shared social networks, careers, schools, socio-economic backgrounds, or interests.
} 
that concerns a group interest, then the decision maker is better informed about how to make a decision that best suits everyone's interests.

Finally, social comparison theory shows that people of any culture tend to be naturally interested in the opinions and progress of others, as they are constantly benchmarking their own behaviours against those of others [42].

Summary of key motivations Situational relevance and interdependence.

Related strategies The underlying motivations of GROUP OPINION are similar to those of GROUP CUSTOMISATION, HARMONY, MENTORING, TEAM PERFORMANCE, SUGGESTION, and SURVEILLANCE. Its motivations contrast with those of REDUCTION, TUNNELLING, and ACHIEVEMENT.

\subsubsection{The MENTORING strategy}

Target audience Members of collectivist cultures.

Antecedents Collectivists are accustomed to the constant presence of other members of their ingroup $[38,51,69,96,142,162]$. This puts the ingroup in the position of observing other group members' actions and behaviour in what is effectively surveillance. In Western cultures, surveillance has negative connotations, although monitoring of one's own behaviours, as in SELF MONITORING, is perceived as acceptable. Collectivists do not feel particularly threatened by surveillance from their ingroup members. They are interdependent with the group members, their identities are intertwined with the ingroup identity, and they are more effective in group situations than situations in which they are required to work alone. In addition, the constant presence of the ingroup acts as a reminder of expected behaviour because behaviour in collectivist cultures tends to be situationally dependent, and motivated by collective responsibility [38, 96, 143]. Furthermore, collectivists have been noted to measure their own performance through the impressions and assessments of other ingroup members [69, 96, 143].

Description The MENTORING strategy involves tracking behaviour that users wish to change, in a similar way to the SURVEILLANCE strategy, but making this information available to other trusted group members. Acting as mentors, the group members use this information to support the users and keep them motivated to change their behaviour. In many respects this strategy could be seen as a distributed form of the SELF MONITORING strategy. 
Real world parallel Genevieve Bell, an ethnographer for Intel, conducted a three year study of the role of technology in Asian cultures. While interviewing members of an Indian family about their technology usage patterns, she discovered that the whole family had password access to their son's email account, and regularly checked and read his email. When she asked the family why they read his email, they replied, "To find out what he is doing! How else will we know?" [19].

Hypothetical PT example: group financial planner Members of the same group account share a goal of paying off their shared debts. They can log on to the group financial planner to review major expenditures entered by other group members, and calculate how they, and other group members are progressing towards achieving the goal. To encourage debt payment over other payments, members can leave each other encouraging notes and messages. Group members can also refer to the account history to identify any obstacles to members' progress to determine who may need more support.

Consequences The MENTORING strategy harnesses the situationally dependent nature of collectivist behaviour $[59,96,143]$. The context of behaviour is explicitly one in which users are aware that they are being monitored by other ingroup members, or mentors, and act accordingly. The role of a mentor is to serve as a positive influence for the person being mentored, and to track this person's progress. The monitoring allows group members to act as each other's mentors. This is akin to existing mentoring programmes, except with the ingroup substituting for a single mentor.

In addition, although users are being monitored by others, given they identify closely with their mentors, the group identity strongly shapes their own identity $[59,69,96,142,143]$. The close level of identification between the user being mentored and the mentors also means that the monitoring is more akin to the more positively perceived SELF MONITORING.

One of the ethical questions related to any surveillance concerns an individual or a group being able to obtain information about another individual or group. The issue is mitigated, however, if the individual under watch welcomes the information sharing, which is more likely to be the case for collectivists, with regards to their ingroup member mentors. Additionally, our interviews showed that collectivists are accustomed to relying on community support mechanisms to enable life changes.

Summary of key motivations Interdependence, group identity, and situational rel- 
evance.

Related strategies The underlying motivations of MENTORING are similar to those of GROUP CUSTOMISATION, HARMONY, GROUP OPINION, DIFFERENCE MONITORING, SUGGESTION, and TEAM PERFORMANCE. Its motivations contrast with those of TAILORING, REDUCTION, TUNNELLING, SELF MONITORING, and ACHIEVEMENT. The motivations of MENTORING are also both similar to, and contrast with motivations of SURVEILLANCE.

\subsubsection{The DIFFERENCE MONITORING strategy}

Target audience Members of collectivist cultures.

Antecedents Collectivists constantly adapt their behaviours and actions to conform with their ingroups, and their behaviour overall is shaped by situational relevance $[69,96,142,143]$. While individualist cultures tend to be loose, collectivist cultures tend to be tight, so there are numerous rigid protocols governing behaviour [142]. As a consequence, differences in behaviour are in principle easier to detect in collectivist cultures than in individualist ones. In order to fit in with the ingroup, then, collectivists are motivated by how they currently do not fit in, and they may seek to find out how their actions differ from group norms. Collectivists do not want to be perceived as different from their ingroup members. Any difference from the group might pose a threat to group harmony, any may be perceived as a desire to not belong to the group [96, 143].

Description The DIFFERENCE MONITORING strategy involves monitoring the actions of users with the aim of achieving a particular task, or adopting certain behaviour. Users are informed if their behaviour differs from preset target behaviours they are hoping to learn, and that are practised by other members of their group.

Real world parallel Conformity is a desirable social value in east Asia. In the Japanese context, it has been described as "the safest protection from criticism and eliminates the need to make personal judgements and errors which can bring quite devastating social consequences" [75]. In a recent study comparing Japanese and American workers' attitudes towards deviation in the workplace, the Japanese workers were more concerned with punishment resulting from nonconformance than their American counterparts. Furthermore, the Japanese work- 
ers perceived a lower level of reward for compliance with workplace rules than did the American workers [86].

Hypothetical PT example: supermarket item scanner Members of the same shopping club have a shared concern to keep their groceries budget at or below $\$ 200$ per week. Club members use their scanners to check how much they are spending on non-essential items, such as ice cream, and compare their spending with other club members. When contemplating buying a particular brand of vanilla ice cream, club members can scan the tub as a potential purchase item. They are then notified whether the price of the ice cream falls within the average range of prices other club members have spent.

Consequences The difference monitoring strategy involves focusing on differences from target behaviours. Given the greater rigidity of societal rules in collectivist cultures, people may be more interested in knowing when they are breaking rules, as opposed to following them. In a rule-dense environment, the likelihood that a rule is being broken is higher (at least initially), so the person stands to gain more if they are notified of what they are not supposed to do.

In addition, because the notification is given by the PT application as opposed to another ingroup member, it facilitates face saving, which is an important feature of collectivism [59, 142]. Group members no longer need to rely on other members to point out what they should be doing and this averts embarrassment for both the person seeking to have their behaviour assessed, and the person they have asked.

From an ethical standpoint, users should have the final say over how and which of their behaviours get monitored, as well as what they deem to be target and non-target behaviours. In effect, this allows users to retain control over which non-target behaviours they are alerted to, and consequently, the kind of interaction they engage in with the persuasive technology.

Summary of key motivations Situational relevance, many rules, group identity, conformity, harmony, and face.

Related strategies The underlying motivations of DIFFERENCE MONITORING are similar to those of GROUP CUSTOMISATION, MENTORING, DISESTABLISHING, TEAM PERFORMANCE, SURVEILLANCE, and SUGGESTION. Its motivations contrast with those of ACHIEVEMENT. 


\subsubsection{The DISESTABLISHING strategy}

Target audience Members of collectivist cultures.

Antecedents The use of praise in individualist cultures is a widespread parenting practice [142]. It is linked to the notion of self-esteem, a prominent concept in individualist cultures, but less important and relevant in collectivist ones $[142,137,38,96]$. Praise is less common in collectivist cultures, and has been associated with leading to excessive pride in individuals [126, 142]. In fact, in cultures stretching from the Mediterranean to India, praise is even viewed as an invitation for attention from the evil eye, a folklore concept which has been described as "responsible for those slow, wasting diseases and nervous or mental disorders for which the untutored mind can find no explanation in the circumstances of the person afflicted" [97]. Rather than use positive reinforcement for teaching behaviour, a noted characteristic of collectivist cultures is the use of punishment [126, 142]. While positive reinforcement focuses on encouraging desirable behaviours, punishment focuses on weakening undesirable behaviours.

Description The DISESTABLISHING strategy concerns training users out of practising specific actions or behaviours that they do not want to perform. It is similar to the CONDITIONING strategy, except that it focuses on the weakening of undesirable behaviours by triggering approved reminder cues when the undesirable behaviour is detected.

Real world parallel Anthropological studies of Tongan and Samoan socialisation practices show that children are not considered full social beings until they have learned proper cultural behaviour (anga fakatonga). Socialisation patterns emphasise learning to surrender personal interests for group interests. Penelope Schoeffel et al. report that this is achieved by shaming children with gentle mockery, ridicule, and teasing designed to teach children to be modest and self-effacing [126].

Hypothetical PT example: music player pedometer The music player pedometer is a portable audio device that plays music and tracks certain types of information about users' movements, like the Nike+iPod product [11]. Users can establish playlists for different types of runs. Users may also opt to use one of the device's preset workout modes, i.e. in fartlek mode, the device will play music tracks of certain tempos at particular times to signal the pace to be running. The device 
also records information about workouts, which can later be uploaded to a personal page on the pedometer website. The personal pages act as blogs detailing users' progress. Users can view each other's blogs to keep up to date with the runs people are undertaking, and to compare their progress.

For example, a user of the music player pedometer is training for a half marathon, and determines that she wants to have completed 25 miles of running by the end of the week. She inputs the goal into the device, and also selects the song Harder, Better, Faster, Stronger to play if she is not on track to reaching her goal. Halfway through the week, she has only completed 5 miles of running. The song Harder, Better, Faster, Stronger automatically gets inserted into her playlists, so that when she hears it, she remembers that she is not reaching her pre-specified target. If she continues to make inadequate progress to her goal, the frequency with which her reminder song gets automatically inserted into her playlists increases, escalating the reminder.

Consequences The disestablishing strategy focuses on weakening undesirable behaviours. The cross-cultural psychology literature shows that collectivists expect to learn proper conduct by focusing on improper conduct [96, 142]. In addition, as collectivist cultures tend to be tight, they feature more rigid rules regarding proper and improper conduct. The key difference between this strategy and DIFFERENCE MONITORING is that it relies on users conceptualising their behaviour less in terms of the behavioural preferences of an ingroup they identify with, and more in terms of how they want to act themselves.

From a Western perspective, this strategy may seem like an ethical quagmire [46]. Rather than arguing for the need to punish, however, we are arguing for the need to be able to focus on undesirable behaviours that users themselves wish to weaken. Furthermore, it is possible to do so without punishment, but by relying on reminder cues. It is important that users are given the option of establishing the form and frequency of the reminder they wish to receive, along with the ability to disable or modify the reminders at any point. As a result, users retain control over their interaction experience with the persuasive technology, and are prepared for what to expect.

Summary of key motivations Punishment and many rules.

Related strategies The underlying motivations of DISESTABLISHING are similar to those of DIFFERENCE MONITORING, and contrast with those of CONDITIONING. 


\subsubsection{The TEAM PERFORMANCE strategy}

Target audience Members of collectivist cultures.

Antecedents Collectivists' self identities are to a large extent informed by the ingroup identity [69, 96, 142, 143]. A close level of interdependence with the group also means that members are motivated to act in ways that uphold the ingroup's reputation. Collectivists generally attempt to act in ways that will save face [51,59], or at the very least, minimise bringing shame on the ingroup [51, 142, 162]. As it is currently used, the SURVEILLANCE strategy tracks the behaviours of individuals, or perhaps a group of individuals, but it generally results in either the promise of rewards for the individual or punishment for the individual [46]. Collectivists, however, not only tend to be more effective in group situations [69, $142]$, but are more concerned with contributing towards ingroup goals $[69,96$, $142,143]$, than individual goals. One of our Māori interview participant summed up this view when explaining Māori and Pacific cultural attitudes: "service to others is more acceptable than looking after yourself first".

Description The TEAM PERFORMANCE strategy concerns tracking the behaviour of individuals forming a group. It rewards or reprimands all members of the group on the basis of the actions of each individual which are related to a group behavioural change goal.

Real world parallel In Bangladesh, the Grameen Bank disburses loans to solidarity groups which are groups of (approximately five) individuals in need of loans. As the individual loans are bundled together, group members must rely on one another to repay their shares before the group can apply for further loans. Dr. Muhammad Yunus, founder of the Grameen Bank, discussed the principle of lending to solidarity groups:

Group membership not only creates support and protection but also smoothes out the erratic behaviour patterns of individual members, making each borrower more reliable in the process. Subtle and at times not-so-subtle peer pressure keeps each group member in line with the broader objectives of the credit program... because the group approves the loan request of each member, the group assumes moral responsibility for the loan. If any member of the group gets into trouble, the group usually comes forward to help [161]. 
Hypothetical PT example: supermarket item scanner Members of the same shopping club decide that they want to buy fewer confectionery and non-Heart Foundation ${ }^{7}$ approved snack-foods because they want to buy healthier food. Using the scanner device, they establish a quota of "treat" items the club members are collectively allowed to purchase each week. As all items are scanned before purchase, each treat item purchased by any club member is recorded. Once the treat quota has been exceeded, club members cannot scan more treat items, indicating that collectively, the club has purchased enough treats for the week. Conversely, by not buying any treat items, shopping club members can conserve the treat quota for another group member, or a future shopping expedition.

Consequences The TEAM PERFORMANCE strategy shifts the ownership of the persuasion goal from the individual level to the group level. It reminds individuals who identify with an ingroup that their combined actions have consequences for the goal outcomes of the whole group. By seeing how their actions can lead to benefits or disadvantages for the ingroup, users might be more motivated to continue acting in support of the persuasive goals of the group. This strategy is likely to be successful for collectivist users, because they are socialised to feel strong affiliations to their ingroups.

While collectivists might be concerned with the welfare of their ingroup from one moment to the next, it is important that the technology clearly identifies to its users which of their individual behaviours affect the group outcome. This strategy also requires clear definitions of groups and group members, so that individuals know whose actions affect them, and vice versa.

Summary of key motivations Group identity, interdependence, and face.

Related strategies The underlying motivations of TEAM PERFORMANCE are similar to those of GROUP CUSTOMISATION, HARMONY, GROUP OPINION, MENTORING, and DIFFERENCE MONITORING. Its motivations contrast with those of TAILORING and SELF MONITORING. The motivations of TEAM PERFORMANCE are also both similar to, and contrast with motivations of SURVEILLANCE.

\subsubsection{The ACHIEVEMENT strategy}

Target audience Members of individualist cultures.

\footnotetext{
${ }^{7}$ The Heart Foundation is a charity organisation in New Zealand that funds cardiac research and promotes healthy lifestyles.
} 
Antecedents Two of the NZ European social marketing stakeholders we interviewed firmly believed in the importance of personal relevance for effective persuasion. The persuasion research supports this finding. People do pay more attention to stimuli if it seems personally relevant [113]. While we can define relevance on individual and group levels, in this strategy we focus on individuals. Personal relevance is more likely to be meaningful to individualists, rather than to collectivists, because of the importance of individual identity [38, 69, 96, 142, 143]. As their reasoning for acting in particular ways is less determined by the situation, individualists also tend to display more attitude-behaviour consistency $[69,96,142,143]$, i.e. they are more driven to avoid cognitive dissonance [42]. For the purposes of persuasion, it could be beneficial to focus on long term personal goals for individualists, because they are both likely to be drawn to the personal focus, and believe that they exert enough independent control over their lives to maintain the changes needed to achieve their goals [96]. A further consequence of ongoing and long-term changes is that their effects can often be perceived by others and, therefore, compared to others. On the whole, individualists have been described as more competitive than collectivists ${ }^{8}$ [142].

Description The ACHIEVEMENT strategy concerns showing users outcomes or consequences resulting from their performance of goal-related target behaviours. It is similar to the TAILORING strategy, but focuses on longer term outcomes, and showcases to others what users have achieved.

Real world parallel Employee of the Month programmes reward employees for performing particularly well. They typically involve an announcement of the recipient in a meeting attended by other employees, a public display of the recipient's name and photo, and the recipient wearing a special badge for the next month. Employees who receive the award may feel validated and, by wearing the badge, they showcase the award to other employees they are implicitly competing against. In order to retain the award and its status, they may decide to continue working in this fashion. Equally, other employees may be motivated by the award to work harder.

Hypothetical PT example: music player pedometer Once a user has completed a run, he uploads the details of his run to a website that maintains a database of users. The website updates his user profile, and integrates the new information in terms of his pre-specified long-term goals, including whether he is meeting or

\footnotetext{
${ }^{8}$ Although it has been noted that inter-group competition, as opposed to intra-group competition, is a thriving phenomenon in collectivist cultures [138].
} 
beating them. He can choose to make his progress information public to other music player pedometer users on the website so that he can compare his results to those of other users.

Consequences The ACHIEVEMENT strategy publicly showcases the effects of TAILORING that are quantifiable or comparable to that of other users. It is persuasive to users as a visual reminder of the attitude or behavioural changes they have adopted and acts as self-motivation for users. Self-motivation has been noted as a typical driver for people from individualist cultures [69, 96, 142].

ACHIEVEMENT also has a competitive aspect. As a marker of accomplishment, or a status symbol, it can track what users have achieved so far, and also what they want to publicly portray. In this way, users can harness competition as a motivator, and implicitly challenge others, drawing on a motivator which is more typical among individualists than among collectivists [142].

Finally, individualists are more likely to be consistent in their attitudes than collectivists, and also to be concerned with consistency because their decision making processes are less situationally-based $[96,142,143]$. They are more likely to be personally concerned with, and independently able to maintain the ACHIEVEMENT, by maintaining relevant attitude or behavioural changes.

Summary of key motivations Self identity, independence, consistency, and competition.

Related strategies The underlying motivations of ACHIEVEMENT are similar to those of TAILORING, REDUCTION, TUNNELLING, and SELF MONITORING. Its motivations contrast with those of GROUP CUSTOMISATION, HARMONY, GROUP OPINION, MENTORING, DIFFERENCE MONITORING, and SUGGESTION. The motivations of ACHIEVEMENT are also both similar to, and contrast with motivations of SURVEILLANCE.

\section{Discussion}

Of the eight culturally-relevant PT design strategies presented, seven of the strategies are developed for use in tools designed for collectivist audiences, while one of them is developed for use in tools designed for individualist audiences.

We note that the collectivist-focused strategies are closely related to each other. Many of them share the same underlying motivations, and in fact, feature areas of conceptual overlap. This indicates that PT designs that are informed by these 
culturally-relevant PT strategies may rely on several of them at once. Fogg observed with regard to his own strategies that in real products, strategies are rarely used in isolation [46].

Figure 4.1 shows a diagram of all of the strategies we have discussed in this chapter. The strategies are arranged according to their underlying motivations. Individualist motivations are represented by squares, while collectivist motivations are represented by circles. Opposing individualist and collectivist motivations (e.g. SELF IDENTITY and GROUP IDENTITY) feature the same colour and horizontal positioning, to denote a relationship.
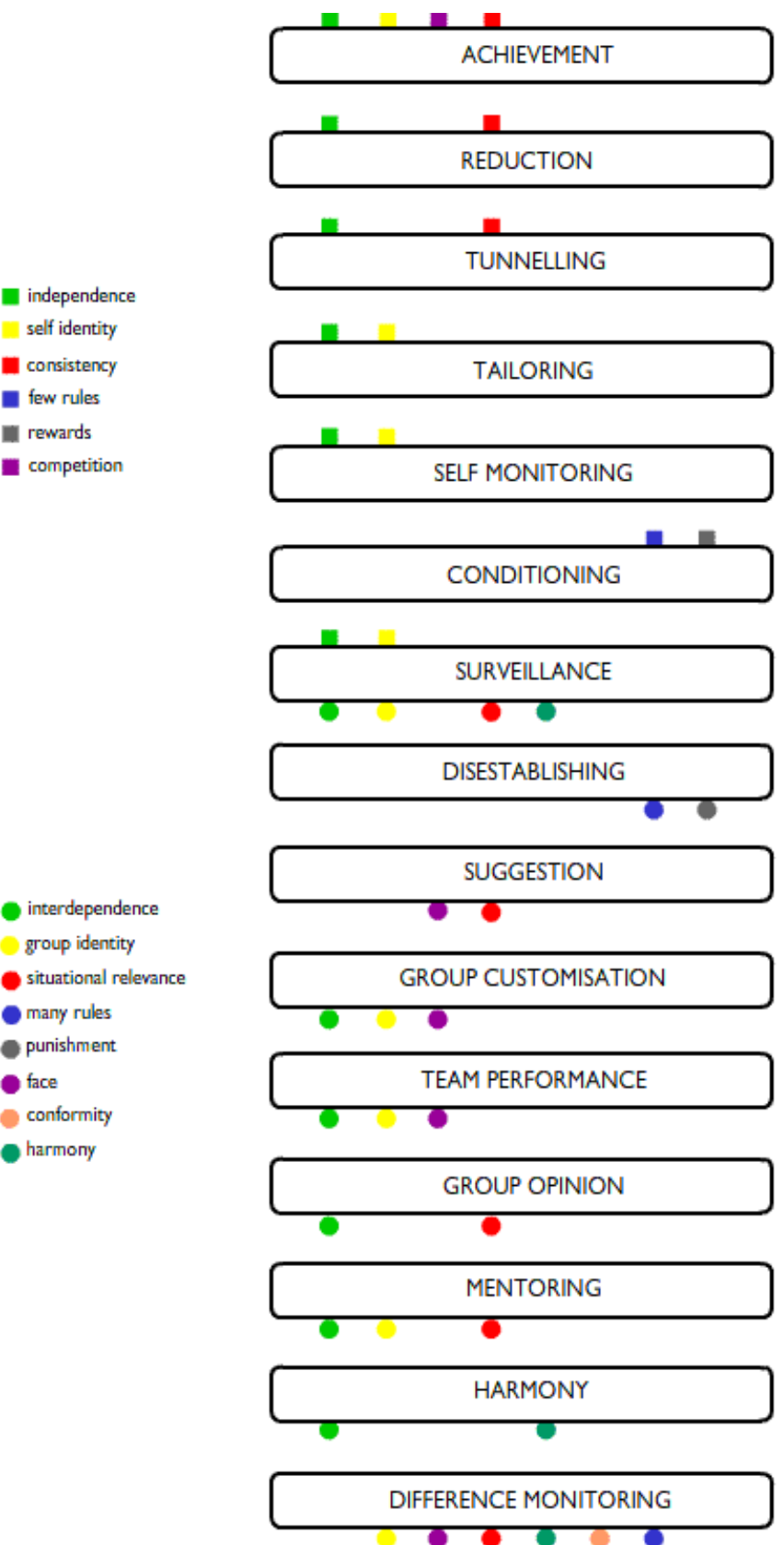

individualist strategies

collectivist strategies

Figure 4.1: A motivation-based arrangement of the PT strategies 
As discussed earlier, several of the collectivist-focused strategies are premised on the existence of a cohesive group, defined and shared group goals, and members who welcome the sharing of personal information that relates to group goals. While this is likely to be the case for collectivists, it may not always be the case. During the design stage, we emphasise the necessity for designers to adopt a participatory design approach. Based on feedback from potential users, designers should clearly identify who will constitute a group profile, and what kind of information users are willing to share with other group members. Moreover, for every application drawing on group-oriented PT strategies, designers should account for users who, after using the persuasive technology for a time, decide to withdraw, and do not want their information to continue to be shared. At the deployment level, users must be clearly informed up-front about what information they will be sharing in using the persuasive technology, and the form and manner in which this information is captured and conveyed.

On a final note, all the design strategies we have discussed, like other PT strategies, could readily be used in tools with unethical purposes. For example, in a collectivist-focused online gambling savings account, offered to users as an easy way to put money aside for gambling. To this end, we urge designers to follow Berdichevsky and Neuenschwander's "golden rule", which we note is individualist in nature: creators of a persuasive technology should never seek to persuade anyone of something they themselves would not consent to be persuaded of [20]. 


\section{Chapter 5}

\section{Smoke? Game mechanics}

A key motivation driving our research was to establish whether a culturallyrelevant PT tool would be more persuasive, more effective, and more welcomed by a target audience, than a tool that was assumed to be neutral. More specifically, we wanted to develop a platform for testing whether our PT design strategies (detailed in \$4.3) provided useful design directions in real tools. With these motivations in mind, we set out to develop a culturally-relevant persuasive game titled Smoke? We developed Smoke? in two versions: one for an individualist, NZ European audience, and the other for a collectivist, Māori audience. While the content, design, and implementation of the two versions differ in particular ways, both are based on the same underlying game mechanics. In this chapter, we present our second research contribution: a pair of culturally-relevant PT prototypes informed by our design strategies. We discuss the rationale underlying our game design process, the common game mechanics of both versions of the game, and overview the functionality of the key game components. In addition, we provide a summary of the features of the narrative common to both versions of Smoke? Throughout the chapter, we include excerpts from a design journal we kept during the development process, as well as screen shots from the final versions of the game.

\subsection{Initial design decisions}

\subsubsection{The starting point}

Many authors have written about the theory of game design (e.g. [34, 121]), and development processes that game designers might consider adopting. Our motivations for game development were somewhat unusual, because we wanted to use the game primarily as a test platform for experimenting with our culturally- 
relevant PT design strategies. Technology constraints were also foremost, as we knew that the game would need to be tested on widely available technologies.

Our first decision concerned the choice of game platform. PCs are more widely available in New Zealand than particular gaming consoles, and people who do not have access to PCs at home often have access to them at school, work, or through city council-provided public computer suites. Consequently, we decided to develop a game that could be played on PCs. In addition, we chose to develop a web-based game, because such games generally do not require much in the way of additional software and hardware support, apart from web-browsers.

Once we had decided to make the game web-based, we opted for Macromedia Flash [92] as the development platform. Macromedia Flash is an authoring tool commonly used for developing interactive web applications, such as website interfaces. The Flash player is a typical plug-in people have installed in their web-browsers. If people have installed the Flash player, applications developed in Macromedia Flash are straightforward to download and launch, either from websites, or as standalone applications. Players of Flash games are not required to download additional gaming software.

Another reason why we chose Flash as an authoring platform was its developmental and programming model. Flash is based on a frame-by-frame movie metaphor. Any Flash application has an overall timeline, and components of a particular frame are displayed (and executed) before components of the next frame are displayed. The underlying frame-based metaphor makes it particularly suited to the creation of animations, which are an integral part of many digital games. In having a simplified process of animation creation, we had the potential to make games of the professional standard gamers expect, with less effort than required by other development tools and environments. An additional attractive feature of the Flash authoring environment was its support for code in the way of ActionScript, its proprietary scripting language. ActionScript was recently extended to be object-oriented (OO), so it now supports features such as encapsulation and inheritance. Although we knew Smoke? would be significantly smaller than many commercial video games, we also knew it would become a quite large project by Flash application standards. The OO extension was very helpful in terms of dealing with complexity.

\subsubsection{Game objectives}

We intended to use Smoke? as an experimental apparatus for our hypothesis by designing one version that would appeal more to NZ European players (which we refer to as the NZE version) and another that would appeal more to Māori 
players (which we refer to as the NZM version). While cultural identity is complex, the cultural assumptions of the NZE version of Smoke? are based on typical attitudes of individualists, and people identifying as NZ European, while those of the NZM version are based on typical attitudes of collectivists, and people identifying as Māori.

Our intention was to make the NZE and NZM versions of Smoke? equally persuasive to their different audiences. Alongside technological considerations, the type of persuasive information communicated to game players was a central factor of consideration in game design. Figure 5.1 shows an early plan from this stage of game development. We established the following as key game objectives for Smoke?:

- to convince contemplative or recent quitters that permanent quitting is desirable.

- to inform contemplative or recent quitters, as well as non-smokers, about types of activities that are likely to facilitate quitting, as well as those activities that are likely to make quitting more challenging.

- to highlight some of the social issues around quitting perceptions.

- to inform contemplative or recent quitters, as well as non-smokers, about typical scenarios in which nicotine cravings can typically be triggered, and what to do about them.

\subsubsection{Determining game genre}

The choice of genre is important in the design process. It effectively determines what kind of interaction the game will afford to players, while establishing conceptual constraints for the designer.

A top-down explanation of game genre is to describe it as collections of common themes connecting well-known games, and conventions that have formed for these types of games over time. A bottom-up description of genre, in contrast, begins with play motivations. Successful games are premised on play motivations that are relevant and meaningful to players. If they were not, people would not want to play them. In this way, genre can be defined as a collective of successful games, relying on shared play motivations.

Broadening the scope of our discussion to consider the role of cultural frameworks, to date there has been scant literature focusing on play motivations from a context of cultural relevance [133]. More specifically, there have not been studies 


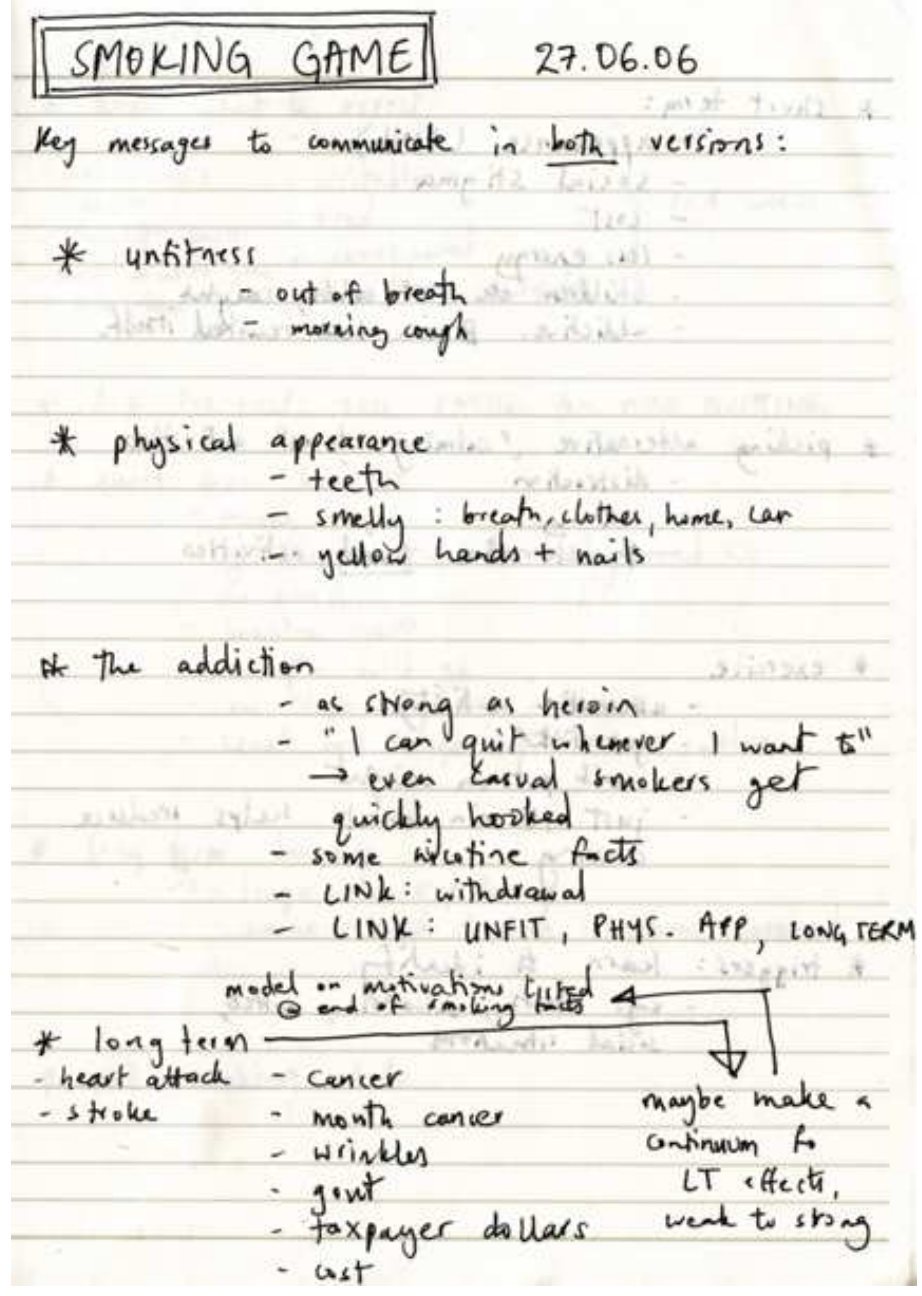

Figure 5.1: An early outline of messages to highlight to players 
investigating genres for individualists, and genres for collectivists. We are not in a position to make the claim that current popular game genres may be better-suited toward individualists, but such a claim does seem plausible. Our objective, however, was not to invent new game genres, but to tweak existing ones to support our research, and more specifically, our game objectives.

On an initial appraisal, our game objectives, given their heavy emphasis on learning as a play outcome, seemed to point us towards choosing the game-based simulation genre. Simulations have been shown to be effective learning environments, with learning from the virtual environment being effectively transferred to real environments [112, 117]. Simulations allow people to experiment with different roles under safe, consequence-free conditions, and they tend to be repeatable. Adopting one course of action rarely reveals the overarching picture, but allowing users to revise choices about courses of action aids in making the underlying rule model more transparent.

The adventure genre of games was another genre with appeal. Adventure games typically place a heavy emphasis on discovery. In turn, this prompts reflection by players on the game's underlying rule structure. An important component of the design objective about beneficial and disadvantageous quitting activities was the idea of players discovering, and then reflecting on cessation advice and craving control activities through the course of playing. Persuasion models indicate that self-discovery facilitates recall and learning [114]. In addition, adventure games typically contain defined narratives. We felt that the use of narrative would support the remaining design objectives, as it would be straightforward to incorporate them into branching game narrative paths. The use of narrative also relates to Bogost's notion of procedural rhetoric, where processes, which might be certain narratives, are used to author persuasive arguments, or outcomes resulting from those narratives [24].

With these reasons in mind, we decided to design Smoke? as a combination simulation/adventure game.

\subsubsection{Conceptual overview of Smoke?}

\subsubsection{Game premise}

In the theme of discovery, we decided to allow our players to experiment freely with both successful and unsuccessful quitting behaviours. The simplest way to communicate the cause-and-effect of cessation-related behaviours on quitting outcomes was to allow players to control the actions of a main character in a game world where the character had just decided to quit smoking. Figure 5.2 


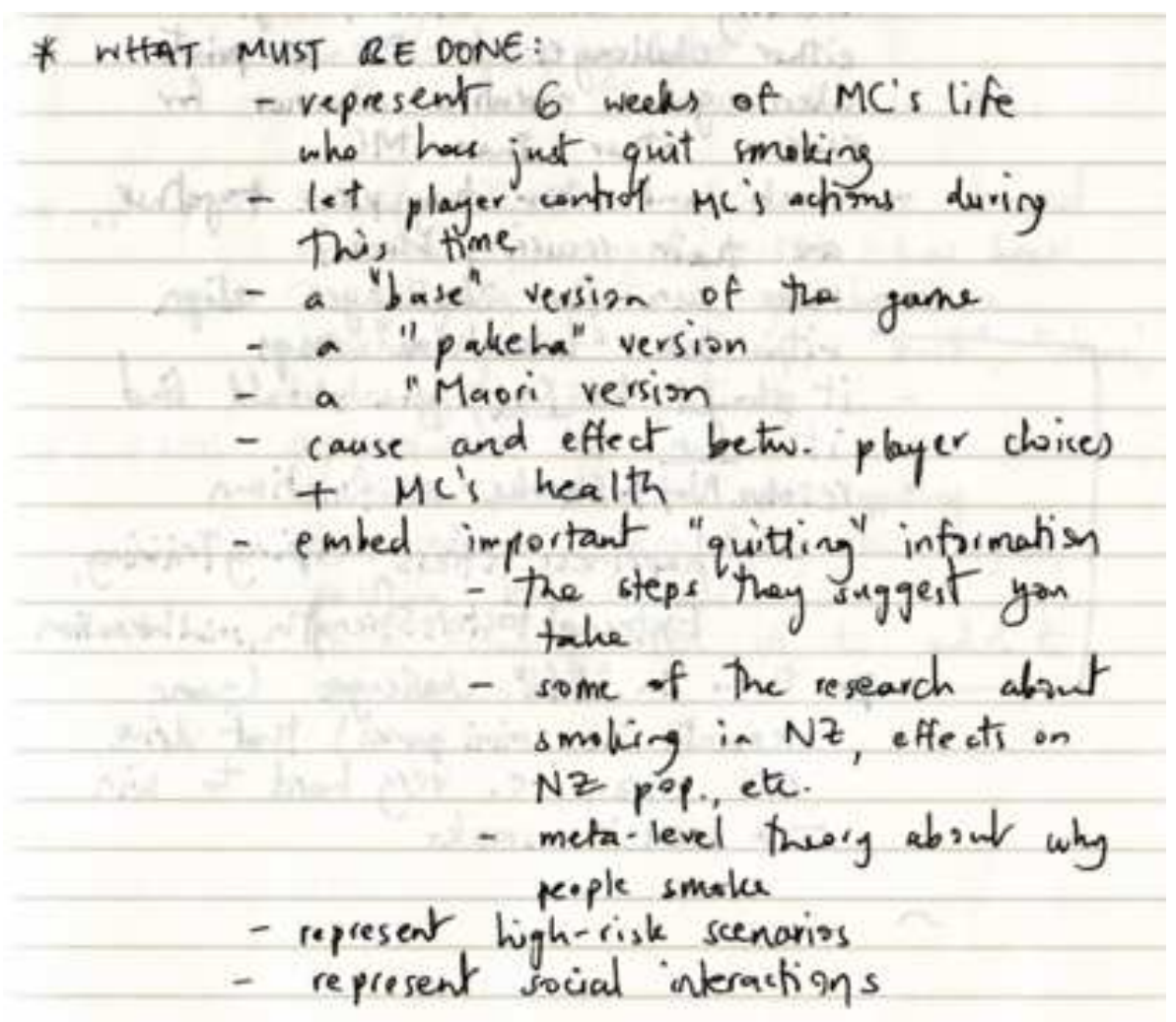

Figure 5.2: Early planning for the game

shows plans we made in the design journal from this early stage of conceptual design.

To take players through a cessation trajectory, that is, from nicotine dependency to independency, we thought that it was key that the main character, who we named $\mathrm{MC}^{1}$, should initially know very little about quitting. In establishing this set-up, it seemed more plausible to introduce the cessation information through the narrative to players, with the design intention being that players might be motivated to learn on behalf of MC. We decided, therefore, that the game would begin under the following circumstances: 19-year old MC (who could be either male or female) would know very little about quitting, would have no specific plan for coping with nicotine cravings, and would not yet have discussed his intentions to quit with his family and friends. Figure 5.3 shows the start of the first scene of the NZM version of Smoke? ${ }^{2}$

\footnotetext{
${ }^{1}$ The name $M C$ refers both to an abbreviation of main character, and the abbreviation for master of ceremonies. MC is also a common nickname in popular culture.

${ }^{2}$ All of the artwork featured in the two versions of Smoke? was created by Rilla Khaled.
} 


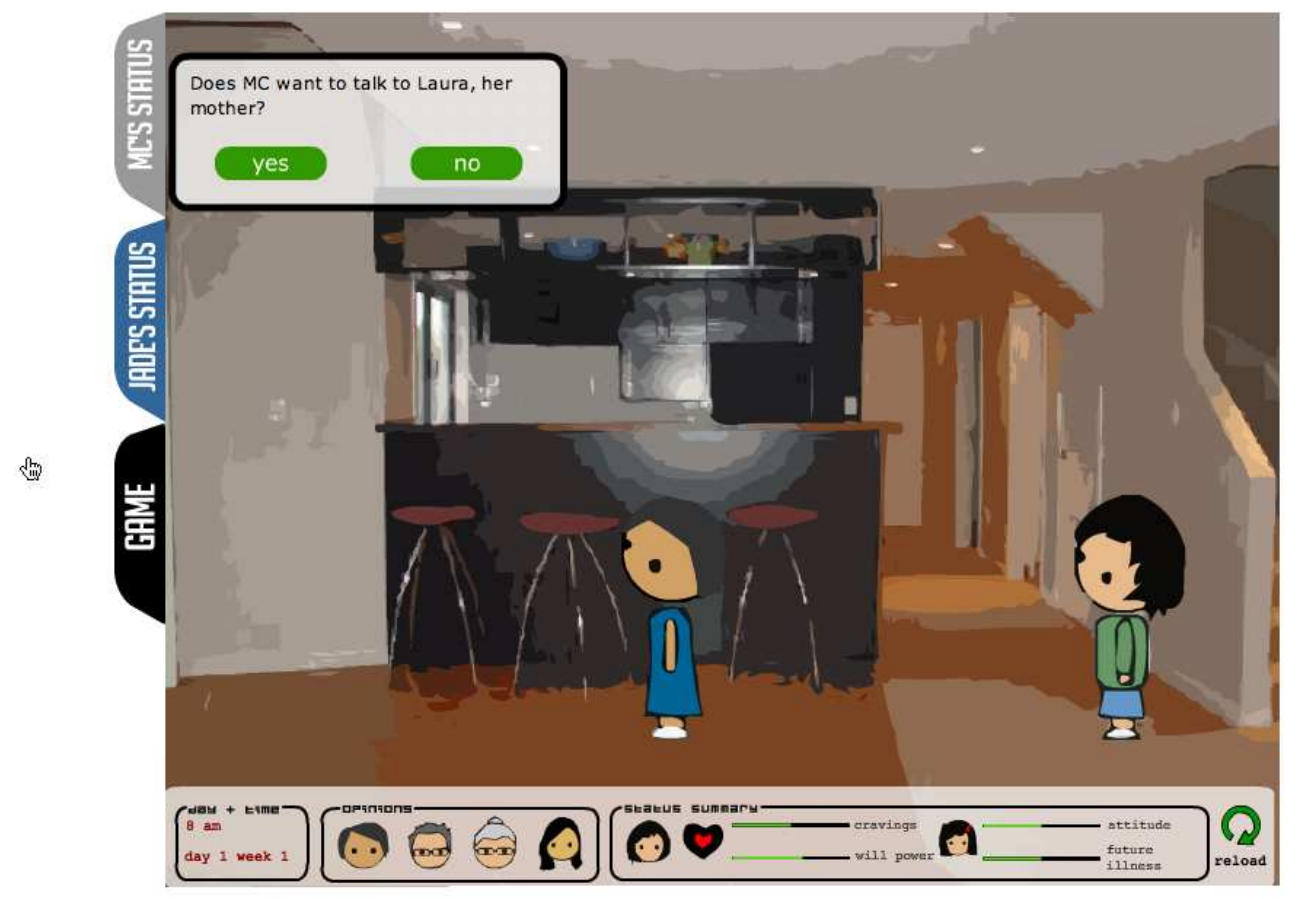

Figure 5.3: $\mathrm{MC}$ and her mother in the dining room

\subsubsection{Player involvement}

To allow players to experiment with quitting behaviours, we decided it would be their responsibility to gradually establish MC's quitting routine, as well as determine how MC dealt with existing and new social relationships. In particular, players would need to decide what courses of action MC would take at decision points. These decision points would include which non player characters (NPCs) MC would interact with, which NPCs MC would attempt to maintain relationships with, how MC would spend his free time, and how MC would handle smoking cravings. As there are different consequences that arise as a result of approaching smoking cessation with varying degrees of positive intention, we decided to make Smoke? a game that could be played more than once. Accordingly, we decided to introduce several distinct narrative paths that players could encounter as a consequence of their decisions for $\mathrm{MC}$, illustrating outcomes resulting from approaching the goal of cessation in different ways. For example, we decided to include one narrative that illustrated an approach to cessation with strong quitting intentions, while another illustrated a cessation approach with very weak quitting intentions. We felt that the distinct narratives supported player exploration and reflection, which are important aspects of learning. All the narratives were to conclude after six weeks of game time, as the cessation 
literature describes six weeks as a reasonable amount of time for smokers to overcome initial withdrawal symptoms, and enter the maintenance phase of cessation [101].

\subsubsection{Game outcome}

At the end of the six weeks, or approximately 30 minutes of real time, we wanted players to be presented with a quit state outcome resulting from the choices they had made during the game. The outcomes needed to include a range of states of cessation, so we determined that players could be faced with one of five outcomes, as a consequence of their game decisions. The outcomes could all be located on a continuum, with one end signifying being definitively quit, and the other end being a regular smoker.

\subsection{Concrete design decisions}

\subsubsection{Game narrative}

As a simulation/adventure game, the narrative of Smoke? was of central importance. We began the process of scripting the game by identifying important events and scenarios that might realistically occur in MC's life over the course of quitting smoking. These included typical events ex-smokers tend to face drawn from the smoking cessation literature, health benefits reaped from quitting, and events that added dramatic interest to the game. They also included anecdotes and situations described by focus groups we conducted with NZ European and Māori participants, which we summarise in the upcoming §6.1. See figure 5.4 for an early outline of the events of the first day of the first week of the game narrative. Following the scenario identification stage, we distributed the events and scenarios over various points in the six week time period. We then identified dependencies between the events. This was important, because it indicated where it was possible to introduce narrative branches, and where we could regroup events. Essentially we were establishing a directed acyclic graph that described the potential narrative paths. This graph would greatly simplify our later checking for narrative consistency and congruency.

From this point, our task was to synthesise much of the narrative into a series of interactions between $\mathrm{MC}$ and the other characters (see figure 5.5), as it was a realistic way for MC to encounter new information about smoking cessation. The bulk of the game writing lay in scripting the conversations between $\mathrm{MC}$ and the other characters. 


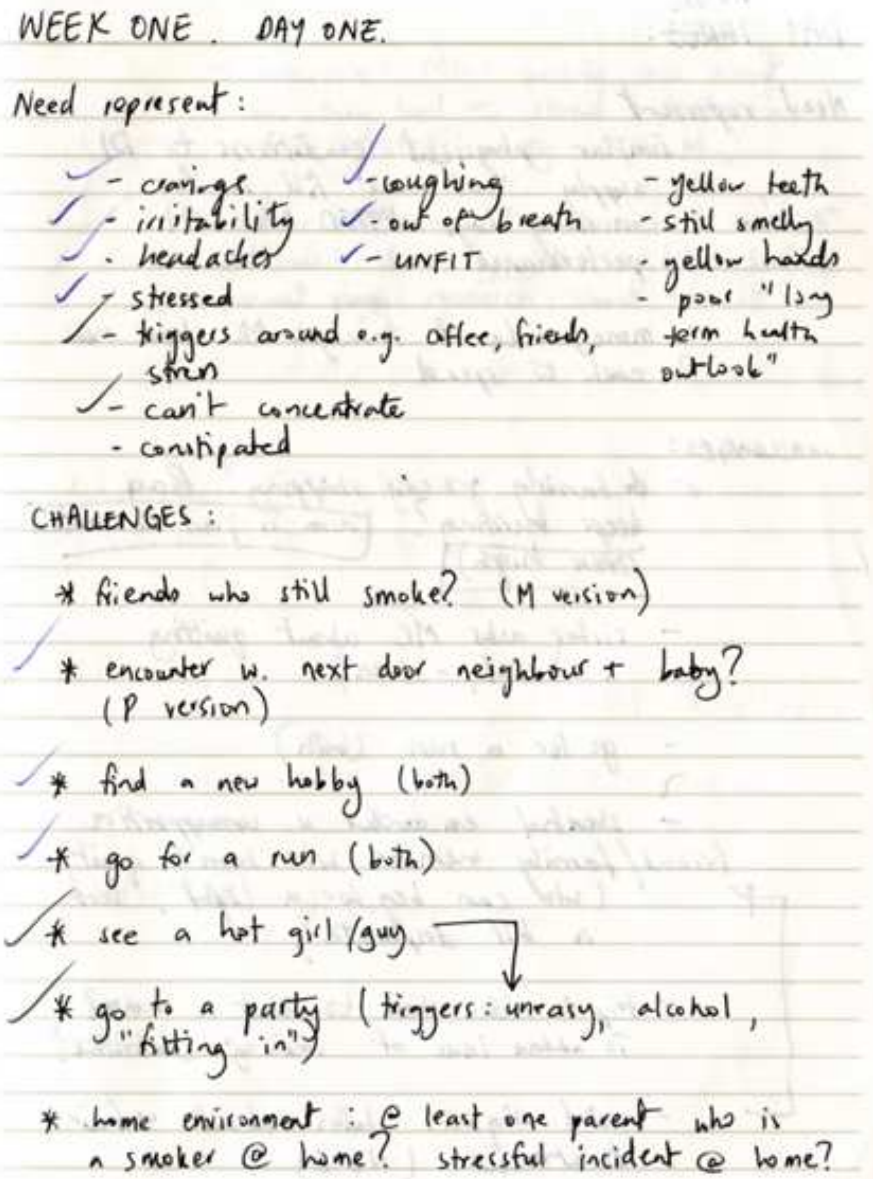

Figure 5.4: A skeleton plan for week 1, day 1

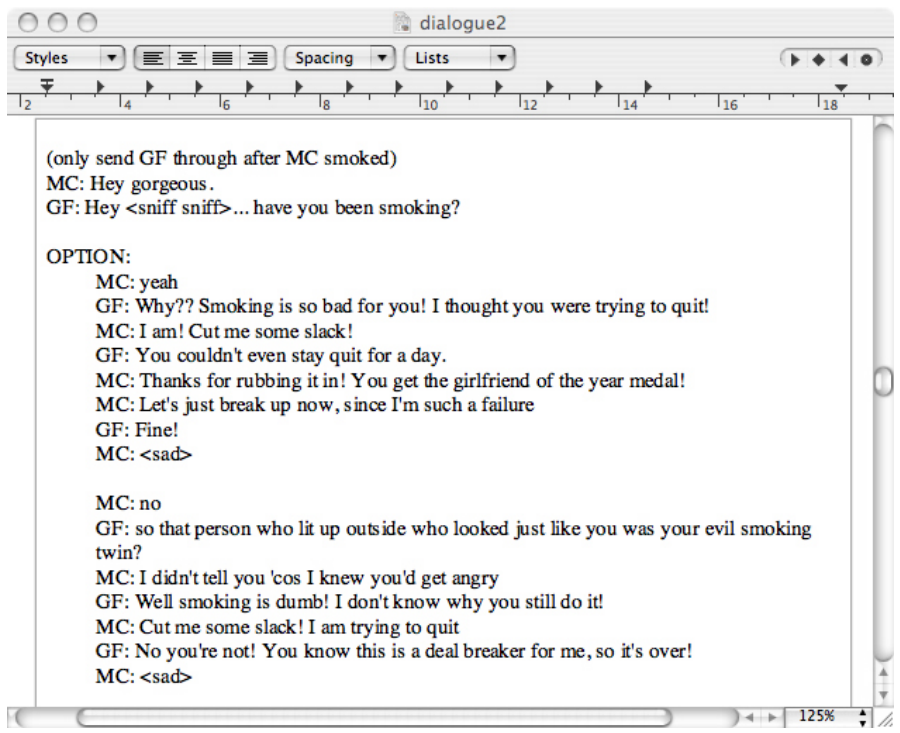

Figure 5.5: Early draft of dialogue from week 1 day 1 of the NZE version of Smoke? 
Since we intended players to direct the conversation flow at particular points, the conversations themselves were written with branching structures. Depending on the branch players decided to choose, the game state could be updated in a number of ways. These included the possibility of $\mathrm{MC}^{\prime}$ 's mood changing, MC or his conversation partner(s) moving or entering a smoking animation cycle, or the conversation partner(s)'s feelings towards MC changing. The game state could also be updated by the remaining contents of the current scene and next scene adapting to align with changes of context.

\subsubsection{Game design concepts}

By piecing together the narrative and conversational dependencies, as well as other cause-and-effect game actions, we began to form an idea of the types of program object structures and game components we needed to implement.

The first stage of program design consisted of identifying key components, clarifying the responsibilities of each component, identifying what data each component needed to access, and determining the relationships between components. Figure 5.6 shows diagrams illustrating how we were considering structuring the phrase components which would collectively comprise a conversation.

The complexity of our design began to grow rapidly. We wanted to harness the $\mathrm{OO}$ mechanisms afforded by ActionScript to simplify our program design, so our next step was to determine how we could use inheritance in the game components.

When we began to code the game, we quickly identified which of our design concepts were feasible, and which ideas were not working with the rest of our components. Here we present the final key concepts of the Smoke? game.

\subsubsection{Scenes}

The overall game concept hinges on unfolding scenarios involving MC. A fundamental game component is a scene, with the game essentially being comprised of a set of scenes. Given the timeline-based narrative of the game, the scenes are related to one another in terms of time, that is, one scene leads to other scenes, and follows an earlier scene, or scenes. The game features two types of scenes. In plot development scenes, the game narrative is developed and MC interacts with other characters. Each plot development scene is comprised of a physical location in MC's life (e.g. his lounge, his bedroom, the garden, a club he visits in the weekends, etc.), the time of day, the NPCs that are present, and potential interactions with each NPC dependent on the context. In wildcard scenes, players are presented with a series of choices for how they think MC should pass a block of time. 


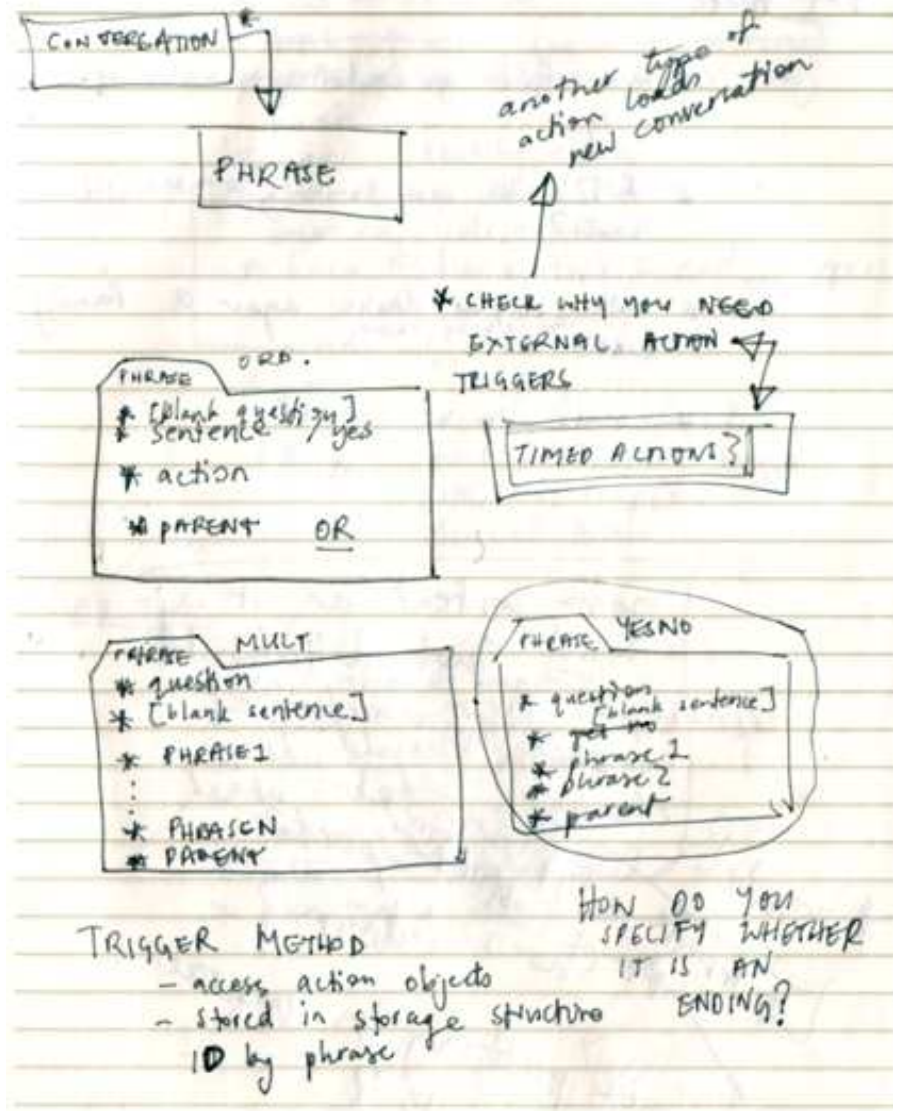

Figure 5.6: An early plan for the phrase component 


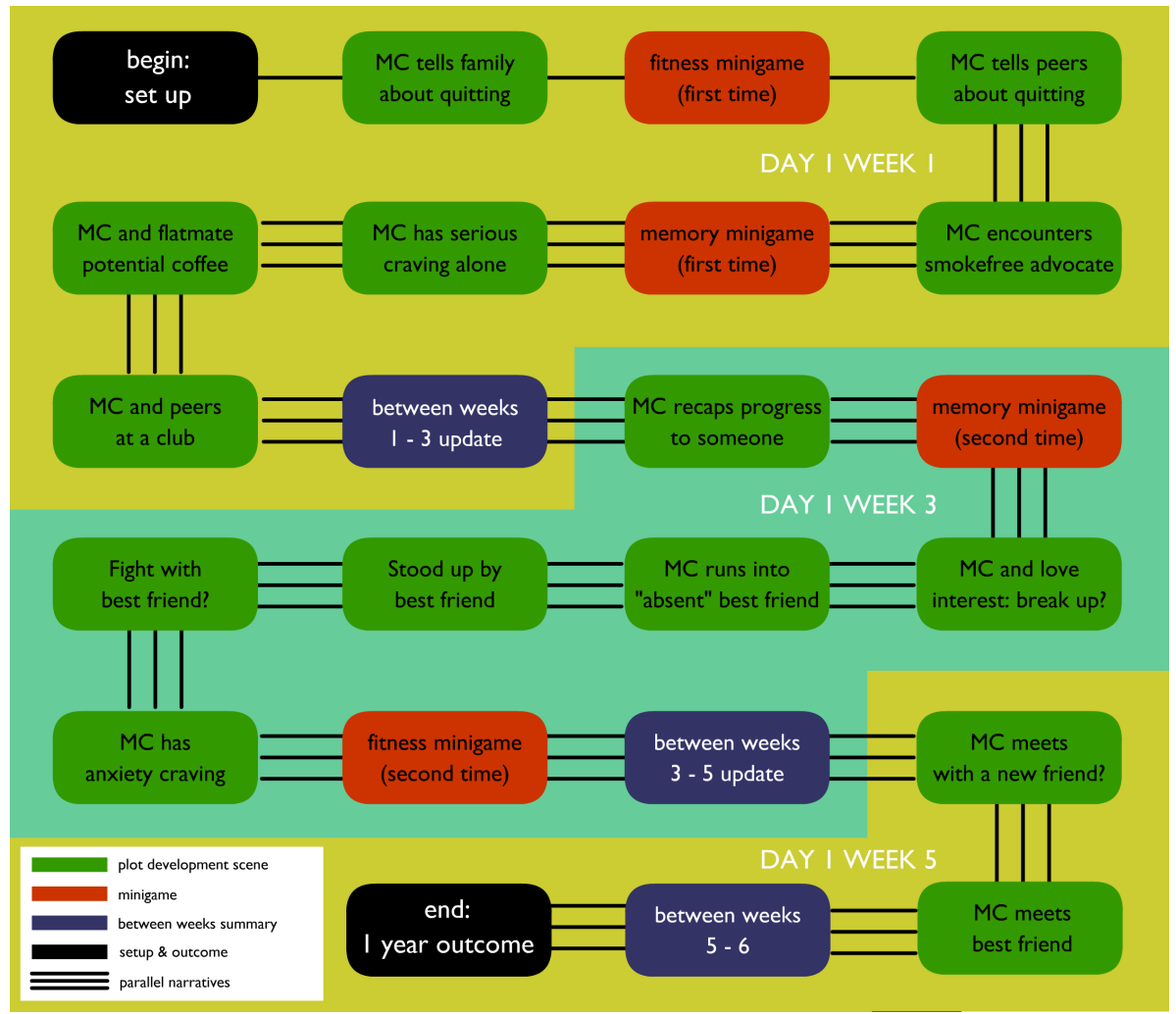

Figure 5.7: A high-level diagram of the narrative of Smoke?

Figure 5.7 shows a simplified, high-level version of the overall narrative of Smoke? Players' decisions for MC determine which version of the narrative will be presented, and also impact on the nature of upcoming interactions with NPCs.

\subsubsection{Characters}

Alongside scenes, characters are a key component of the game. The majority of the game narrative is progressed through interactions between MC and NPCs. The NPCs can converse with each other and also keep track of which conversations they have taken part in, as well as the direction that the conversations took.

In any particular plot development scene, if players choose to make MC interact with an NPC, MC's future relationship with the NPC is affected based on the nature of the interaction. Accordingly, the contents of future plot development scenes featuring the same NPC are also adapted. If players choose not to make MC interact with an NPC and the NPC is pivotal to the narrative, MC's avoidance of interaction is noted, and future interactions between MC and the NPC in question are correspondingly affected.

Although the narrative is largely driven by character interactions, it is not required that players make MC converse with all NPCs. Decisions about who players want $\mathrm{MC}$ to maintain relationships with are an integral feature of the 


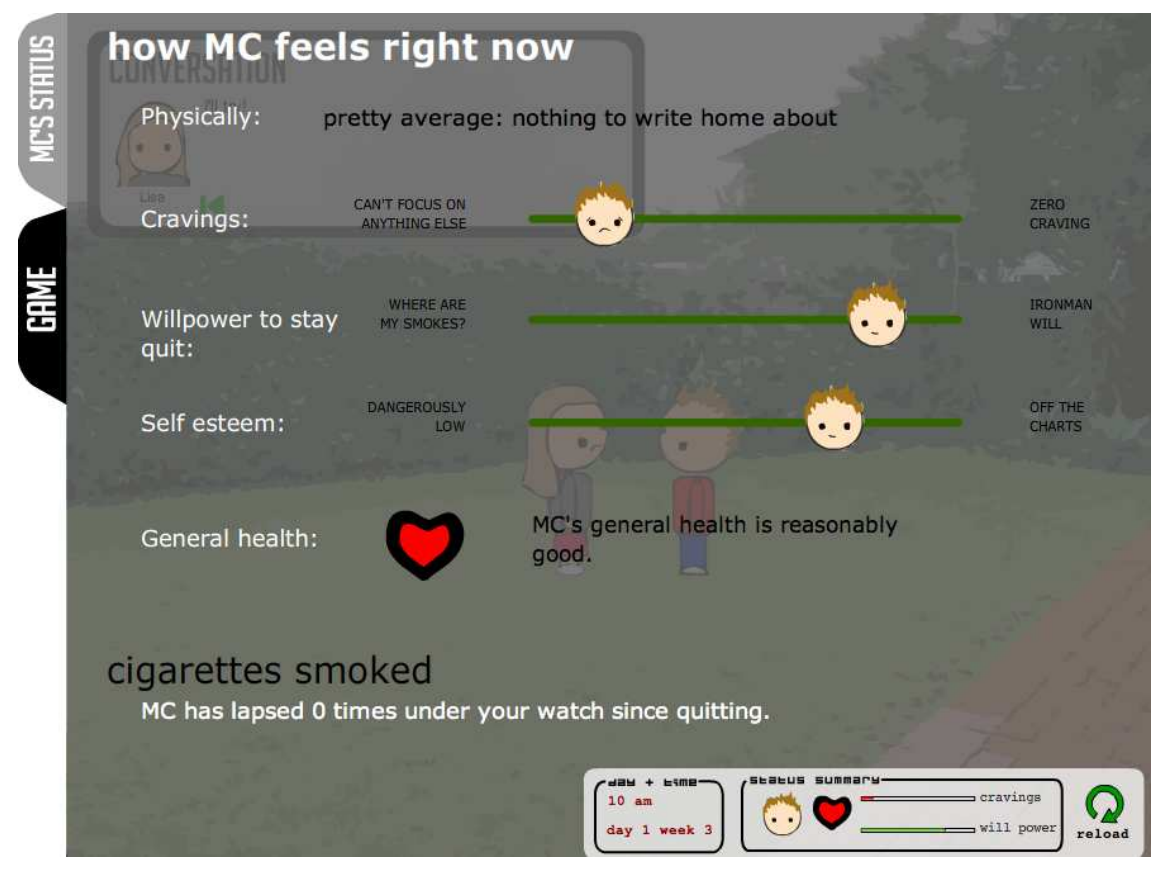

Figure 5.8: The NZE version of the status screen

explorational play experience. Each plot development scene therefore features NPCs with whom it is necessary to engage in at least some level of interaction, if only to specify that MC does not want to converse with them, before it is possible for players to proceed to the next scene.

Status screen From the number of lines cleared in Tetris to a Sim's level of satisfaction in The Sims 2, games constantly present score-based evaluations of performance. We wanted players to be able to track MC's progress throughout the game and additionally, we wanted to highlight how $\mathrm{MC}^{\prime}$ 's current health and wellbeing was being affected by players' choices. Both versions of the game feature a well-being status screen that reports on five aspects of $\mathrm{MC}^{\prime}$ 's current physical and emotional well-being. It also includes the number of times $\mathrm{MC}$ has lapsed, by smoking a cigarette. Figure 5.8 shows the NZE version of $\mathrm{MC}^{\prime}$ 's well-being status screen.

\subsubsection{The cigarette craving mechanism}

An important aspect of both versions of Smoke? is the representation of the addictive nature of nicotine, and how smokers often continue to suffer from cravings after they have quit. In addition, we wanted to illustrate the habitual nature of smoking, and how the urge to smoke arises even in the absence of physical addiction [101]. To understand what might cause MC to crave a cigarette at any given time, players can consult the ever-present well-being status screen. As well as the 


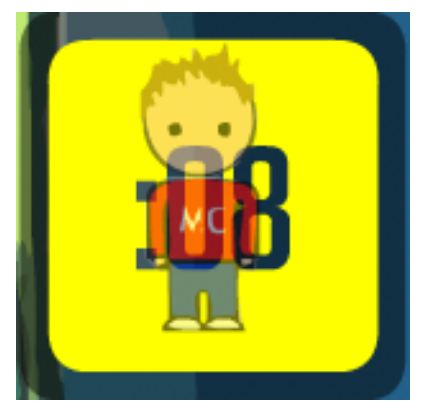

Figure 5.9: The countdown box

individual well-being factors, the game also determines an overall state for MC. This is an average of the five physical/emotional factors represented on the status screen. When MC's overall state drops below a threshold, MC enters a craving cycle, indicated by the presence of the countdown box, shown in figure 5.9. The cycle culminates in MC smoking if players do not quickly adopt a craving control activity for MC to carry out.

The cessation literature indicates that people who have adopted coping activities in advance have a greater chance of managing their smoking cravings than people who have not established such coping activities [107]. To emphasise this idea to players, MC has no established craving control activities at the start of the game. Player can opt to drain MC's willpower to tide him over if a craving occurs, but once MC's willpower has been drained, it cannot be used as a craving control mechanism until some other event has given $\mathrm{MC}$ a willpower boost. Supporting the theme of discovery, during the course of play, it is the responsibility of players to discover, adopt, and collect craving control activities. Figure 5.10 shows a plot development scene from the game in which players are invited to add phoning $M C^{\prime}$ 's love interest as a craving control activity.

When MC next has a craving, the newly adopted craving control activities are presented to players as alternative courses of action. Certain activities, however, are not always suitable for the type of craving $\mathrm{MC}$ is experiencing. For example, a social anxiety-triggered craving may not be resolved by carrying out an activity that alleviates boredom-triggered cravings. Each activity, therefore, has a craving context in which it is most likely to be effective. Figure 5.11 shows a situation in which MC has had a craving that was "caught" by the player, but this craving can only be resolved by talking to someone.

\subsubsection{Wildcard scenes}

We had intended Smoke? to have an average play-through time of approximately half an hour. This seemed long enough to provide players with a brief overview 


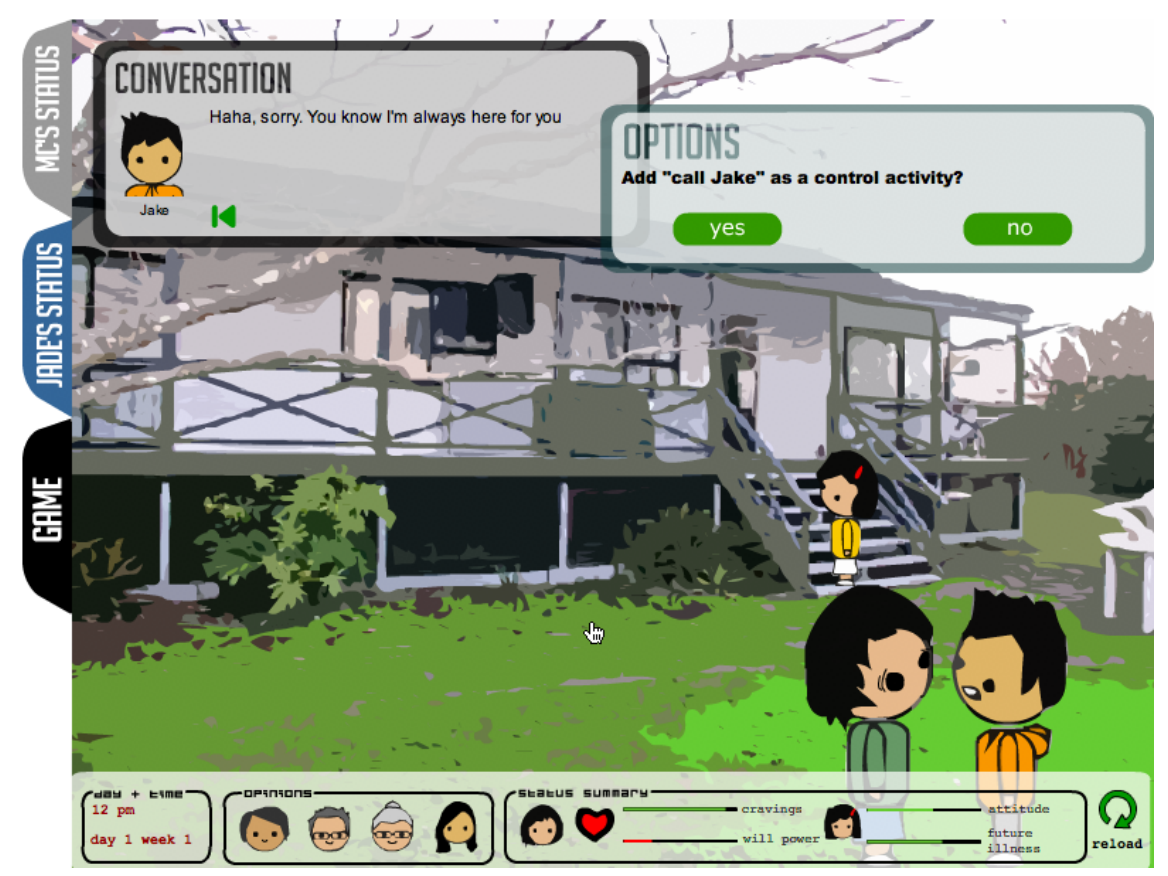

Figure 5.10: Option to add "call Jake" as a craving control activity

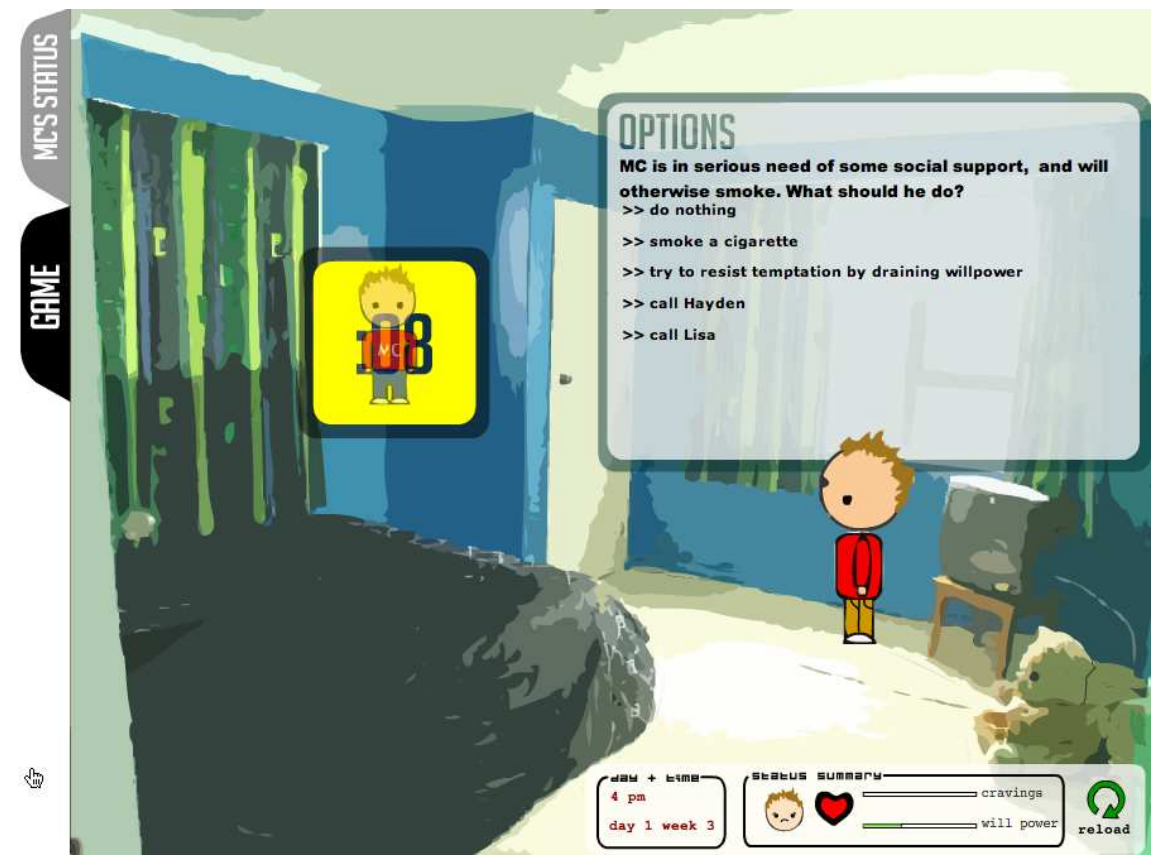

Figure 5.11: Options of craving control activities when social support is a craving context 


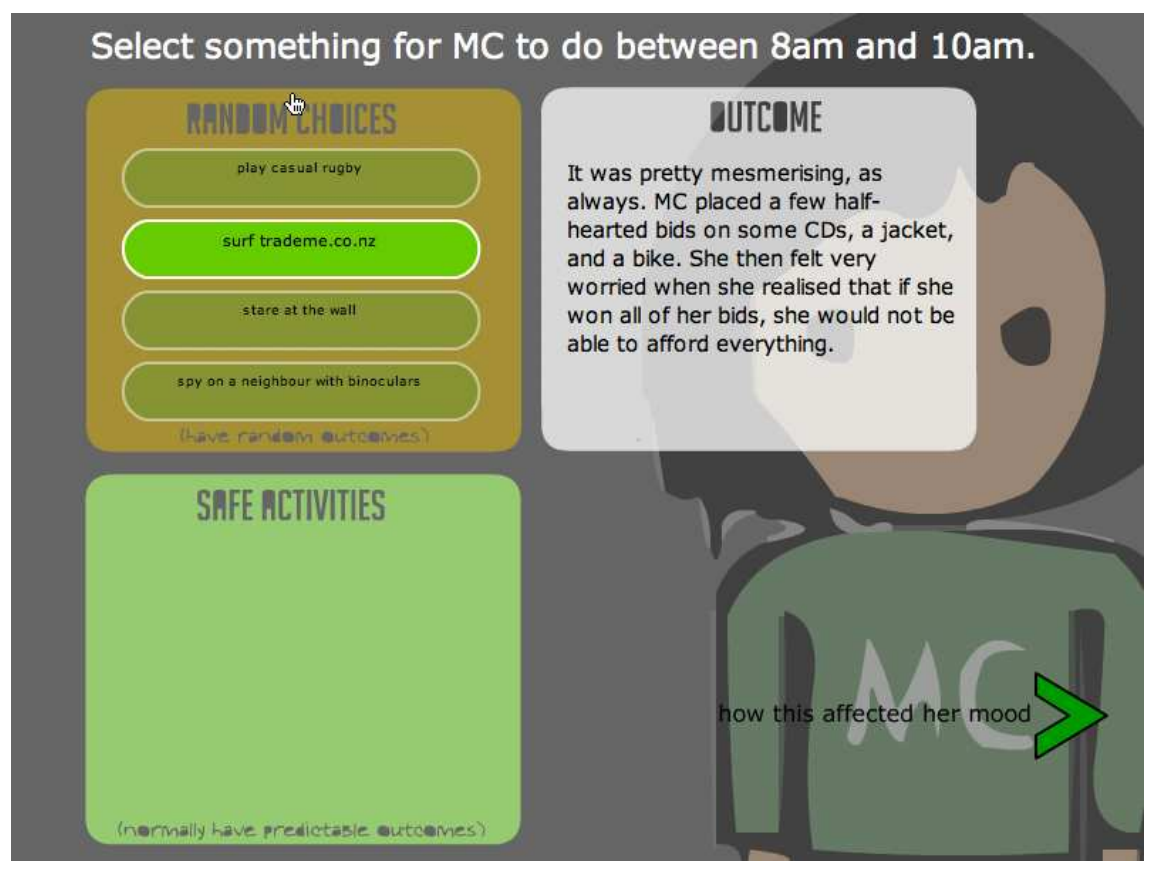

Figure 5.12: Wildcard scene outcome

of the cessation process, while being short enough not to lose their attention. Further, as the game was web-based, we felt that 30 minutes was as much time as we could reasonably expect players to remain engaged with a web-based, social marketing game. Accordingly we limited the amount of interaction-driven narrative content of Smoke? as the interactions are time-consuming. To progress the story of MC's day quickly, with players still feeling as if they are in control of MC's actions, we introduced wildcard scenes. In wildcard scenes players are presented with a series of choices for how they think MC should spend the next two hours. On committing to a particular choice, players are shown an outcome for what happens after MC carries out the activity they have chosen, as in figure 5.12.

The outcome can affect MC's physical/emotional state. For example, after playing a game of rugby, MC might feel happier, but physically worn out. The outcome may also have an overall detrimental effect on $\mathrm{MC}^{\prime}$ 's physical/emotional state, to the point of dropping $\mathrm{MC}^{\prime}$ 's overall state below the craving control threshold. In this case, the next plot development scene will begin with $\mathrm{MC}$ in a craving cycle which players need to deal with.

\subsubsection{Minigames}

To allow players to experience a change in MC's physical/emotional state as a result of quitting, we included minigames within the main game. The purpose of the minigames was to demonstrate how smoking has limited some of MC's capabilities. One of the minigames focuses on $\mathrm{MC}^{\prime}$ s ability to concentrate, as 


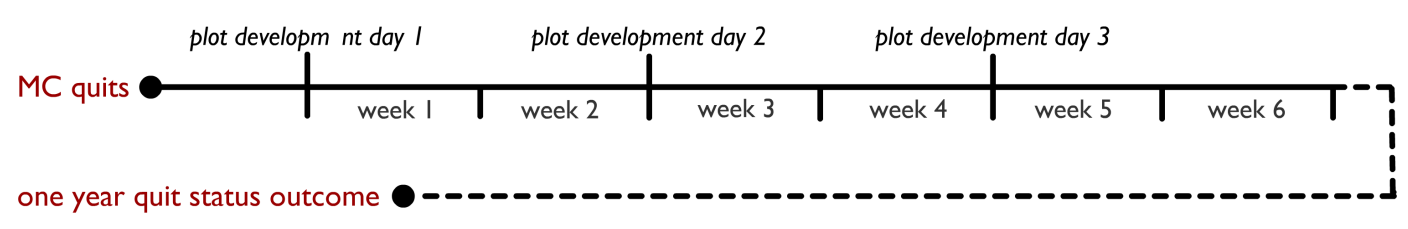

Figure 5.13: A timeline for the narrative of Smoke?

research has shown that two of the initial withdrawal symptoms of quitting are forgetfulness and an inability to concentrate [131]. The other minigame takes the form of a physical challenge, because fitness is an aspect of well-being that is usually compromised for smokers.

We designed two different minigames for each version of Smoke? In the NZE version of Smoke?, the concentration minigame consists of helping MC during a shopping expedition (see figure 6.9), and the fitness minigame is about MC going on a run (see figure 6.3). In the NZM version of Smoke?, the concentration minigame concerns making sandwiches for other family members (see figure 6.16), and the fitness minigame involves players helping $\mathrm{MC}$ to play tag with his cousins. We discuss the rationale underlying the different contexts of the minigames in $\S 6.2$ and $\S 6.3$

Unlike the rest of Smoke?, the minigame challenges are time-bounded. They also rely on additional forms of interaction, such as keyboard input, but there is no narrative decision making. Each minigame features in the overarching narrative twice, and any improvements in $\mathrm{MC}^{\prime}$ 's capabilities are dependent on players' efforts to maintain a smoke-free lifestyle for MC.

\subsubsection{Between weeks summaries}

Much of the game's narrative arc takes place over three specific plot development days: the first days of weeks one, three, and five, with each week starting from Saturday. As the plot development days occur over the weekend, MC is never in classes, or at work ${ }^{3}$. Figure 5.13 shows a timeline of the game narrative. An advantage of spacing out the plot development days over the six week period is that changes in $\mathrm{MC}^{\prime}$ s physical/emotional well-being are clearly visible to players. A disadvantage, however, is that short of supplying players with a description

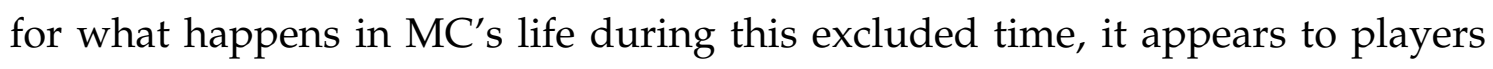
that MC's life stagnates in two week blocks. While we decided against providing summaries of narrative updates (deciding such omissions were acceptable), the game does present summaries of how MC's health and well-being changes over

\footnotetext{
${ }^{3}$ We note that for many smokers, breaks from work are a typical opportunity for smoking. We chose not to set scenes of Smoke? in work-places, because it would have increased the time taken to play.
} 


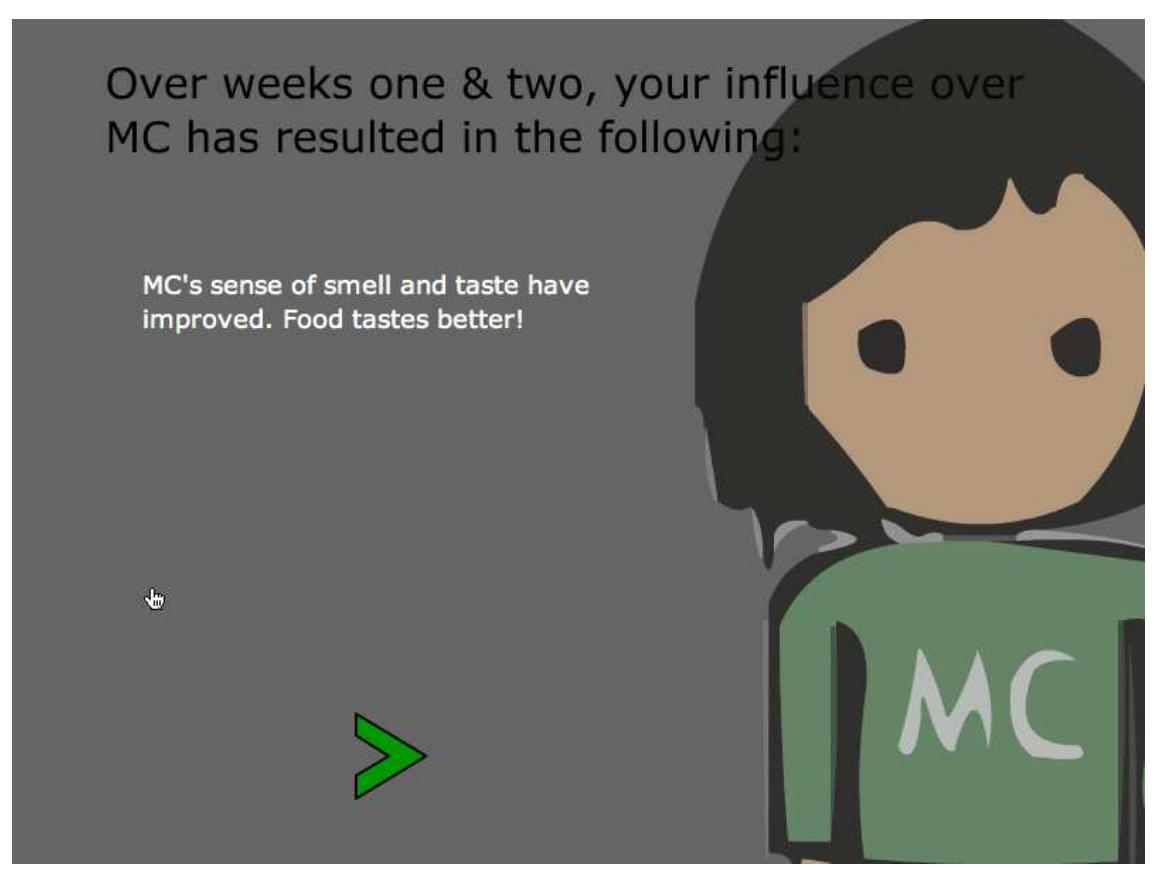

Figure 5.14: Update of stagnant time between weeks 1 and 3

each two-week block (see figure 5.14). This is a realistic use of time as cessation of smoking yields significant health benefits relatively quickly.

Importantly, players are only notified of improvements in $\mathrm{MC}^{\prime} \mathrm{s}$ health and well-being if they have attempted to set up ways for MC to cope with cravings, if they have not intentionally made MC smoke, and if they have not allowed MC to lapse. If they have not achieved these milestones, they are presented with information about MC's ongoing health concerns.

\subsubsection{Game outcome}

While the game spans only six weeks, we do present players with an outcome that reflects $M C^{\prime}$ s quit state one year on (see figure 5.15). $M C^{\prime}$ s outcome is a function of several factors, including how many times players allowed MC to smoke, how many times MC lapsed because players were unable to find a suitable alternative activity, and how many craving control activities players adopted. MC's physical/emotional state fluctuations have an important influence on $\mathrm{MC}^{\prime}$ s well-being over the course of the game, because they are directly related to $\mathrm{MC}^{\prime}$ s smoking cravings. We decided, however, that as long as MC's overall status was consistently brought back to manageable levels, the physical/emotional state should not impact on $\mathrm{MC}^{\prime}$ s one year quit outcome.

Although the one year outcome is in fact derived from how players handle $M C^{\prime}$ s first six weeks of cessation, we decided that players might feel more responsible for MC's outcome, and more motivated to learn about MC's successes and 


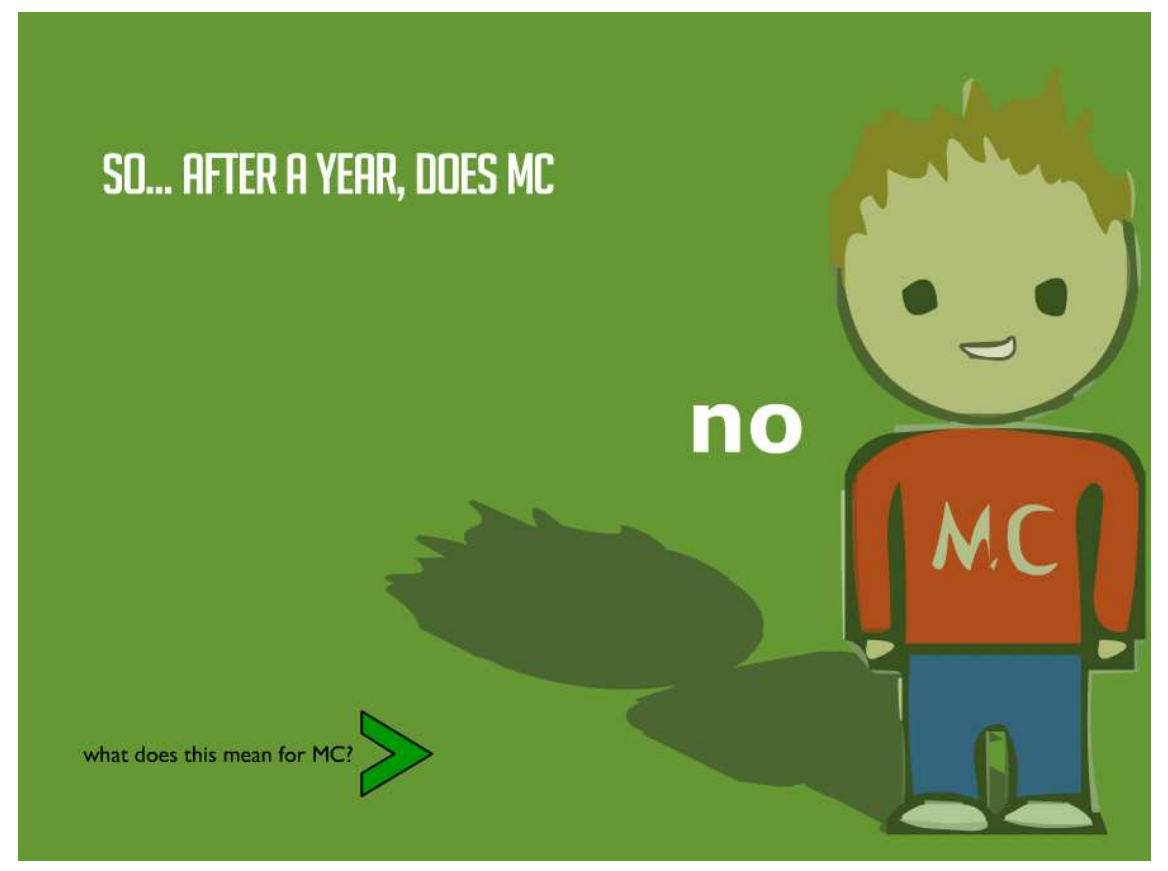

Figure 5.15: A one year outcome for the game

failures, if they felt that they had a long term influence over MC's future. Additionally, we believed that presenting players with a six week outcome would not seem very significant, and in fact it is difficult to definitively characterise someone's quit state after just six weeks. Six weeks indicates the beginning of what cessation experts term the maintenance phase of cessation. 


\section{Chapter 6}

\section{Smoke? Incorporating culture}

A defining feature of a culturally-relevant PT tool should be that its persuasive approach is well-received by and effective on its target audience. As explained in $§ 3.3$, smoking is a serious health issue in New Zealand. It was important that the persuasive approaches and contents of the two versions of Smoke? reflected knowledge and attitudes on smoking cessation in an informed, relevant, and meaningful manner. We decided that it was necessary for us to investigate what smoking meant to people in the NZ context, especially from NZ European and Māori perspectives. In this chapter, we continue our discussion of the initial design phase of Smoke? We focus on how we incorporated culture into the design of the game versions and summarise our findings from focus groups conducted with NZ European and Māori participants on the topics of smoking and social marketing. We then show how the focus group findings informed our application of the PT design strategies in the two versions of the game, and also discuss how the findings influenced the game contents at a more general level.

\subsection{Focus group insights}

We conducted two focus groups with NZ European and Māori participants, the methodological details of which we discussed in \$3.3.2.2. We discuss here the central findings that emerged from analysis of the transcripts of the sessions. The following discussion is structured primarily by the themes that emerged from each group, and secondly, by stances held by the group members. Where possible, we have related our findings to the cross-cultural psychology literature. 


\subsubsection{Willingness to engage with social marketing}

The NZ European participants While the NZ European group was generally welcoming of social marketing messages, a few stated that they disliked seeing realistic violence:

I don't know if I want "social psychological" between TV breaks... I loathe those ads ${ }^{1}$, especially the violent ones... I think they're meant to make us react because they're quite strong, but I find them more violent than dramatic violence, so I quite resent them in my ad breaks.

Furthermore, nearly all of them said that they had become adept at tuning out social marketing messages:

When you're aware of it being an ad, I think it affects your response. You go "it's just an ad...", "it's a tool...", "that someone's trying to convince me of something..." so that kind of breaks the verisimilitude.

The Māori participants In contrast, the Māori participants expressed more interest in social marketing advertisements than commercial advertisements because they believed the information was both relevant and useful:

I know that because I'm Māori, there's a higher chance that they're gonna affect me. There's a greater chance I'll have cancer, that someone in my family will have cancer, so I take that on, whereas I know that someone who's trying to sell me Coke probably doesn't care that I'm Māori or not, or like expensive luxury products, they probably aren't selling to me.

At the same time, the Māori participants voiced a desire to see a more balanced and mature depiction of Māori culture and language in the NZ media:

...we can tell you got told that you have to put in some percentage of Māori words, so they've incorporated it into the show all wrong and random... you could just imagine the director going, "Ready, set, go!" and then "Mate, that was great!" and you can just imagine that they did it thirteen times, like "Kia Ora", no "Kia Ora." Now they use it willy nilly!

\subsubsection{Taking up smoking}

The NZ European participants A major reason stated for adopting smoking as a habit was that smoking is still perceived as "cool":

\footnotetext{
${ }^{1}$ Social marketing advertising in New Zealand can be graphically violent.
} 
I associate it with old, glamorous movie stars... if you smoke gracefully, it looks really cool.

It's still this slightly rebellious thing... perhaps, you know, "I'm living life incredibly intensely, I'm gonna die but I'm just so cool".

By freely discussing the concept of "coolness", the NZ European participants demonstrated familiarity with thinking in terms of universal and consistent ideals, as opposed to group-level, and situationally-relevant norms. Individualists are more concerned with consistency in attitudes than collectivists, whose attitudes are situationally-dependent [96, 143].

Smoking was also described as a social ice-breaker and as an escalation of work-related social smoking. One participant stated that he took up smoking as a means to quell anxiety.

The Māori participants In contrast, while the Māori participants could think of examples of "cool" smokers in the media, they did not cite coolness as a reason for taking up smoking. Instead, they began smoking to fit in with their friends:

When I first started smoking, it was not because it was cool but because so many of my friends do... now I don't smoke because it's cool, I just do.

Furthermore, several of them claimed that so many of their family members were smokers that smoking had become an implicitly acceptable behaviour:

Seeing it like it was normal, it was easier for me to pick up, but for my friends, whose parents didn't smoke, [they] weren't into smoking. It was a big deal for them, whereas for me it was just a smoke.

Unlike the NZ Europeans, the Māori participants were less concerned with universal ideals, and more interested in ingroup norms, supporting what the crosscultural literature suggests about attitudes of collectivists [38, 69, 96, 143].

\subsubsection{Smoking as a habit}

Both groups A reason for continuing smoking suggested by participants in both groups was that all people have bad habits, and that smoking was less risky than other habits. They added that they were aware of the negative consequences of smoking. Both groups also discussed how youth smokers, in particular, believed themselves to be impervious to the consequences of smoking. 
The Māori participants The Māori participants also described their smoking as a rebellion against the control exerted by their families on other aspects of their lives:

With Māori people, especially, you've got so many responsibilities as it is that you want something for yourself.

The perception of smoking as an act of rebellion implies both that the Māori participants were strongly aware of their responsibilities to their family and that, under normal circumstances, they were concerned with upholding ingroup expectations. This reflects what the cross-cultural psychology literature proposes as typical values of collectivists [59, 69, 142, 143].

When discussing whether cost was a deterrent, the Māori participants described how it was still relatively easy for them to obtain cigarettes in spite of financial hardship:

I could probably ring up my mum and go "Oh, could I have 20 dollars for smokes?", and she'd send it.

There's always someone around who's got a cigarette.

In collectivist cultures, the concept of sharing with ingroup members is both emphasised and expected $[69,96,142,143]$. Australian research on smoking in Aboriginal communities explicitly cites a "culture of sharing" as a key reason for high rates of smoking among Aborigines [31].

\subsubsection{Locus of smoking}

The NZ European participants When they talked about the sphere of influence extended by a smoker's habit, which we refer to as its locus, it was mainly discussed in the context of a personal habit that was the choice, the right, and the prerogative of the smoker to address individually. On the topic of spending money on cigarettes, one participant said:

In New Zealand, because we're quite comfortable with the old, I want to say "protestant", work ethic, like you work hard, make your own life, you save, you invest, we' re quite comfortable with the idea of individualised benefit and cost, and you make decisions based on what is good for you.

The focus on the individual is typical of individualist societies, and less typical for collectivist societies [38, 69, 96, 142].

On the topic of setting examples for others, another said: 
If I was unhealthy because of smoking, I would hope my kids would be able to see me as a negative role model instead of as a positive role model, and I'd be able to say, "Don't do this kids, it'll screw you up", and that would have an effect.

What is interesting here is that the participant seemed to be reasoning about how role modelling functions. It is possible that she had been raised in an environment in which positive role modelling to younger family members was not emphasised as important.

The Māori participants In contrast, Māori participants brought up other people in relation to smoking frequently. In fact, the Māori group more or less agreed that smoking is a cultural norm that affected many of their family members:

With smoking, in my family, it's a given. You're granted the permission to smoke because everyone else does too.

When suggesting ways to convince young people not to start smoking, one Māori participant jokingly suggested telling them:

It's not cool! Even though your aunties ${ }^{2}$ and your uncles who you all love do it, it's not cool!

It is interesting to reflect on how this participant's suggestion denotes an expectation of love and respect for extended family members, as well as a normal desire on behalf of younger family members to emulate older family members. It also illustrates a tension between respecting elders and not wanting to learn by their example.

During discussion, the Māori participants used the term "role model" frequently. When asked what it meant, they gave a variety of responses, including:

I've got responsibilities to youngsters in the family, like I've messed up, I've made heaps of mistakes, but I'm kind of a role model as well so I'm really aware of the things that I do because I know I'm being watched.

It's so hard to figure out why I'm a role model but I know I am one. I've got little cousins... and I know their parents, and I know they look up to me, but I know that I drink, I swear, I smoke, and all of that, and lots of it in front of them, so I don't know.

\footnotetext{
${ }^{2}$ In Māori culture, the title auntie may refer to any female elder, and does not necessarily indicate a family relation. This is similarly true of the title uncle, while the title cousin may refer to a contemporary, or someone younger in age.
} 
It's like, "Don't do that because you're the big sister". To me, that's like, "Don't do that, you're a role model", because you're being reminded of your position, which is kind of like a role. "Don't do that, you're the oldest grandchild". "Don't do that, you're the big sister". "Don't do that because we can see you".

Role modelling is based on the important collectivism concepts of conformity, harmony, and hierarchy $[51,59,143,162]$. People are reminded of desirable behaviour through their group responsibilities and their social ranking within the group.

\subsubsection{Reasons for wanting to quit smoking}

Both groups Both groups described diminishing fitness as a motivation for wanting to quit smoking:

I quit smoking for health reasons primarily because I started losing my fitness and feeling like shit... I didn't have any problems with it at all until I started to feel shit and I went, "well if I don't stop then I'll feel worse".

Physical appearance was also a motivation for the women in both groups, who were concerned with the visible effects of smoking on their skin, lips, and teeth:

The smoking ads where they show stuff happening to people on the outside, like people ageing prematurely, and getting all wrinkled and crusty, or yellowed teeth, I thought those are good ones because they appeal to people's immediate sense of vanity. You look in the mirror and those are going be your teeth that you see.

Referencing an NZ advertisement that shows tar-clogged lungs:

You know those ladies with crusty lips.. you know... when you go to kiss them, and they screw their mouths up, and they put lipstick on and it bleeds... well that meant more to me than the lungs.

The NZ European participants They talked about how they believed society did not generally accept smoking, or smokers, and had, in fact, progressively stigmatised them:

Society has been quite effective in addressing people's reactions... if you smoke, the reaction is "Gross!" It's a bad fault in the smoker that they're smoking. 
It's like smoking is automatically bad and unhealthy.

Once again, the NZ Europeans were reflecting on abstract and stable societal beliefs which seem to be more common in individualist, rather than collectivist cultures $[96,143]$.

The Māori participants They did not bring up societal attitudes towards smoking, but the smokers among them did talk about how their smoking habit led to feelings of exclusion. Referring to smoke-free entertainment events, and the recent legislation change in New Zealand in which bars and restaurants became smoke-free zones:

You go to those events and they're smoke-free, like even outdoors events, it sucks!

Especially with not smoking in pubs, when you do go outside, it's like "you're a smoker!"

Both of these participants referenced the concept of social exclusion. Exclusion from groups is considered to be harsh punishment in collectivist cultures $[69,96$, 142, 143].

The Māori participants often discussed how they were aware of the effects of passive smoking, and how they did not wish to inflict them upon others, especially younger family members:

If I walked into a room and saw my little cousins sitting around a table and people were smoking around them, that would make me give up. Smoking, that's my own choice, I deal with it, that's my own problem. But how can you give that to your little cousins? Your blood? I realised that when I was young, I can put myself in that situation, and it didn't do me any good.

Clearly the Māori participants were willing to look to other family members as motivation for quitting smoking. Motivation derived from other ingroup members is typical of collectivists [69, 96, 142, 143].

\subsubsection{Opinions on characteristics of effective appeals}

Both groups All participants talked about how they appreciated advertisements that referenced elements of NZ culture, including symbols, rituals, and humour. Both groups thought that appeals referencing health and outward appearance would be effective in getting people to think about quitting smoking, but were split over the issue of whether positively or negatively framed appeals would be more effective. 
The NZ European participants Almost all of them felt strongly that quitting permanently was ultimately an issue of personal willpower over physical addiction, and that it was something that someone could achieve on their own:

If you really want to stop, like if you actually want to stop, you will by yourself I reckon.

Both my flatmates gave up smoking at the same time. The person who did it for themselves with no support just quietly made their way to a point where they felt they could announce it several days later once they'd achieved some mileage. But the one who made lots of noise was smoking again the same day.

These attitudes indicate that the NZ European participants believed that people have autonomy and control over their own choices and actions, which is more in line with individualistic, rather than collectivistic perspectives [69, 96, 142, 143].

When asked about future life goals, and what would be most effective in getting smokers to think about life in the long term, the NZ European participants agreed that not everyone wants children, and that personalisation of goals would be more effective than global appeals:

You could insert your own incentive at the bottom, "I want to live long enough to afford a Ferrari Testarossa", that might be whatever works for you in the future.

This is an example of personal customisation or TAILORING, and we already discussed in $\S 4.2$ how TAILORING is more suited to individualists than collectivists.

The Māori participants They frequently brought up other family members as the reason for staying healthy:

To me living a long life is being around to watch my grandchildren.

The Māori participants also thought that age would be a significant factor in how different types of appeals would be perceived:

I don't have any kids and I don't plan on having any for a very long time, but if my mum was looking at "I want to be around to play with my grandchildren", it would probably be most effective on her.

For the ads, they usually pick someone pretty haggard looking in their forties, which makes sense to me, but as a teenager, that's so far away. Maybe you don't talk about lungs when you're young, but instead, "you have to stand outside all the time", "you always smell when you walk into a room and everyone knows you've smoked". 
Regarding the effectiveness of cessation appeals from other family members, the Māori participants said they would be unwilling to listen to elders, because it seemed insincere coming from other smokers. They did, however, state that they would listen to appeals from younger family members:

I don't think the adults would ask the adults to stop smoking, but if the kids would ask the adults, that would make a difference to me.

Noteworthy here is that the Māori participants illustrated that they were willing to be motivated by the wishes of other family members, which is common in collectivist cultures [96, 142, 162].

\subsection{Incorporating insights into the design}

\subsubsection{Insights into design: common features}

The focus group findings discussed established that there were some opinions and attitudes regarding smoking cessation and persuasion held by members of both the NZ European and Māori groups. We discuss now how we harnessed these common focus group findings in the content of both the NZE and NZM versions of Smoke?

\subsubsection{Referencing local culture}

"Whenever you see the haka on TV, it's like 'Awww yeah"' Both groups strongly agreed that they enjoyed seeing NZ culture referenced in the media. A guiding factor in establishing and determining Smoke?'s contents was for them to connect with the local. In both versions of Smoke? we used backdrops of quintessentially NZ suburban environments, such as house-fronts, neighbourhoods, and gardens. Figure 6.1 shows one of the backgrounds. In addition, although the avatars in the game are very simple visually, we designed their clothes to appear typically New Zealand, e.g. several characters are clad in hoodies ${ }^{3}$ and shorts.

The language contents of the game also reference NZ culture. For example, the game conversations make use of local and youth-oriented vernacular. In addition, the craving control activities presented to players are those suggested to us by teenagers from the Wellington region and members of Victoria University's Toihuarewa, the Māori academic forum.

\footnotetext{
${ }^{3} \mathrm{~A}$ hoodie is a hooded sweatshirt.
} 


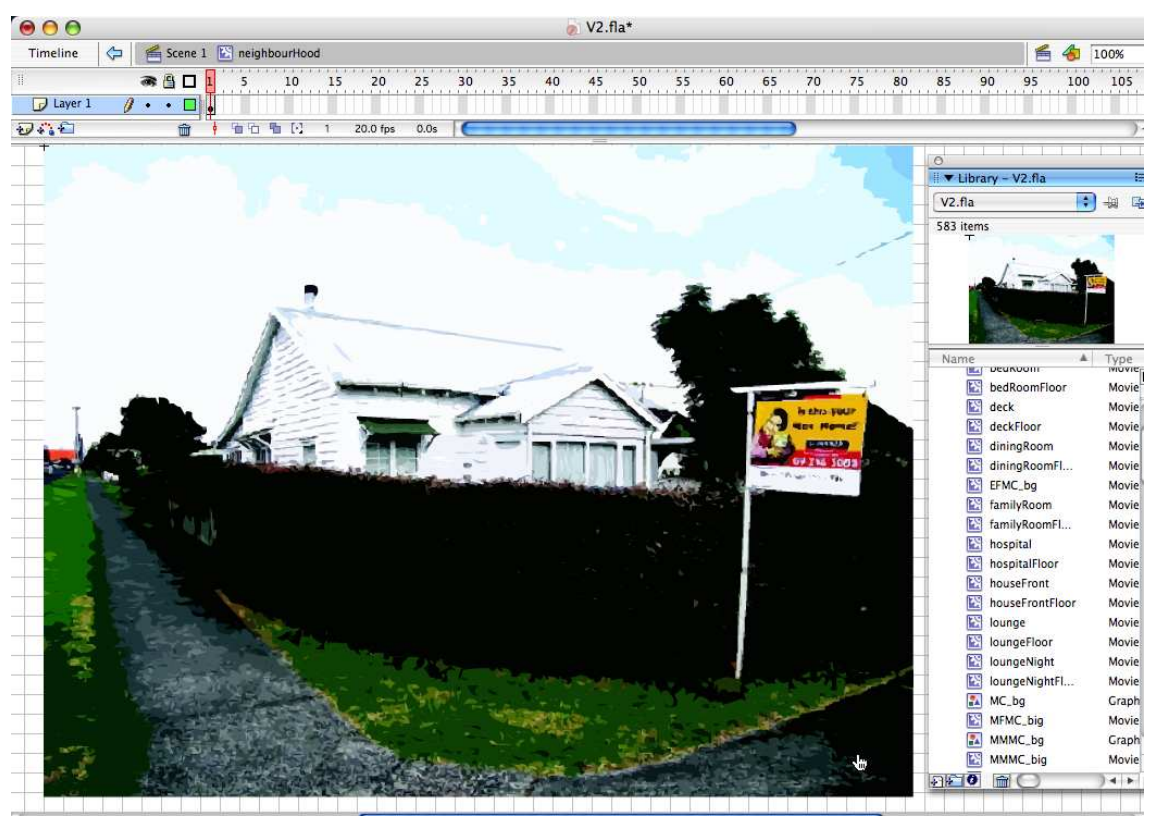

Figure 6.1: A neighbourhood background in the Flash development tool

\subsubsection{Fitness-based cessation motivations}

"If I don't stop then I'll feel worse" Discussion from both focus groups indicated that everyone was aware of the serious long-term health effects of smoking. We decided that health effects would therefore be a relevant and important aspect to highlight in the game content. At the start of both versions of Smoke?, MC has poor fitness. This reflected comments made by some of the smokers in the focus groups, regarding how they had already noted reductions in their fitness as a consequence of smoking. We decided it would be useful for players to track how MC's health was changing in response to their choices during the game. Accordingly, the status screens, which can be viewed at any point during play, present information about MC's current health and well-being. Figure 5.8 shows the NZE version of MC's status screen.

A further way in which we hoped to encourage players to consider the negative health effects of smoking was by the representation of unappealing aspects of smoking. During the morning hours of the early weeks of quitting, MC has a constant "smoker's cough". The animation for the cough dramatically twists and contorts MC's otherwise neutral face. We also designed characters' smoking animations to look mechanical, to reference the cyclic nature of addiction. After the smoking animation, characters who have smoked are enveloped in a hazy cloud, representing the persistent cigarette smoke odour that many smokers complain about. 


\subsubsection{Insights into design: the NZE version}

\subsubsection{The notion of 'cool'}

"It looks really cool" In the NZ European focus group, there was consensus that smoking is perceived as "cool", and we were clear that this concept had to be explicitly addressed in the content of the NZE version of Smoke? The narrative of this version features characters, such as the best friend, who are smokers and considered "cool", but as a counterpoint, it also features other characters who are similar to the smoker characters, and are explicit about their status as nonsmokers.

\subsubsection{Stigma against smoking}

"It's like smoking is automatically bad and unhealthy" Society-level stigma was cited as a motivation for quitting among members of the NZ European focus groups. This motivation is harnessed in the narrative of the NZE version of Smoke? in two ways. The literature on smoking cessation motivations shows that an important motivation is people's concern for their perceived attractiveness decreasing because they are smoking [21]. Primarily for this reason, both the NZE and NZM versions of Smoke? feature a character who is a love interest for MC, but who is an ardent non-smoker. MC's love interest is relatively unsympathetic to MC's struggle with quitting, and over-simplifies the quitting process.

A recent legislative change in New Zealand has resulted in smoking being banned from many public areas. This change was welcomed by many non-smokers, reinforcing society-level opposition to smoking. Referencing this attitude, the narrative of the NZE version of Smoke? includes another character, who is very negative about smoking. She is a friend of $\mathrm{MC}^{\prime}$ 's family and is very opposed to people smoking in the presence of her baby. She is judgemental when she initially encounters $\mathrm{MC}$, as she knows that until recently, he had been a smoker. Figure 6.2 shows a screenshot displaying part of this interaction, from the NZE version of Smoke?

\subsubsection{An individual responsibility}

"If you actually want to stop, you will by yourself" The NZ European group findings highlighted their strong belief that quitting smoking is the responsibility of the smoker, and under the smoker's own control. In the NZE version of Smoke?, certain characters strongly encourage MC that he can quit, and reinforce quitting as a desirable goal. The theory of planned behaviour links people's likelihood of carrying out a particular behaviour with their perceived self-efficacy [8]. Gulliver 


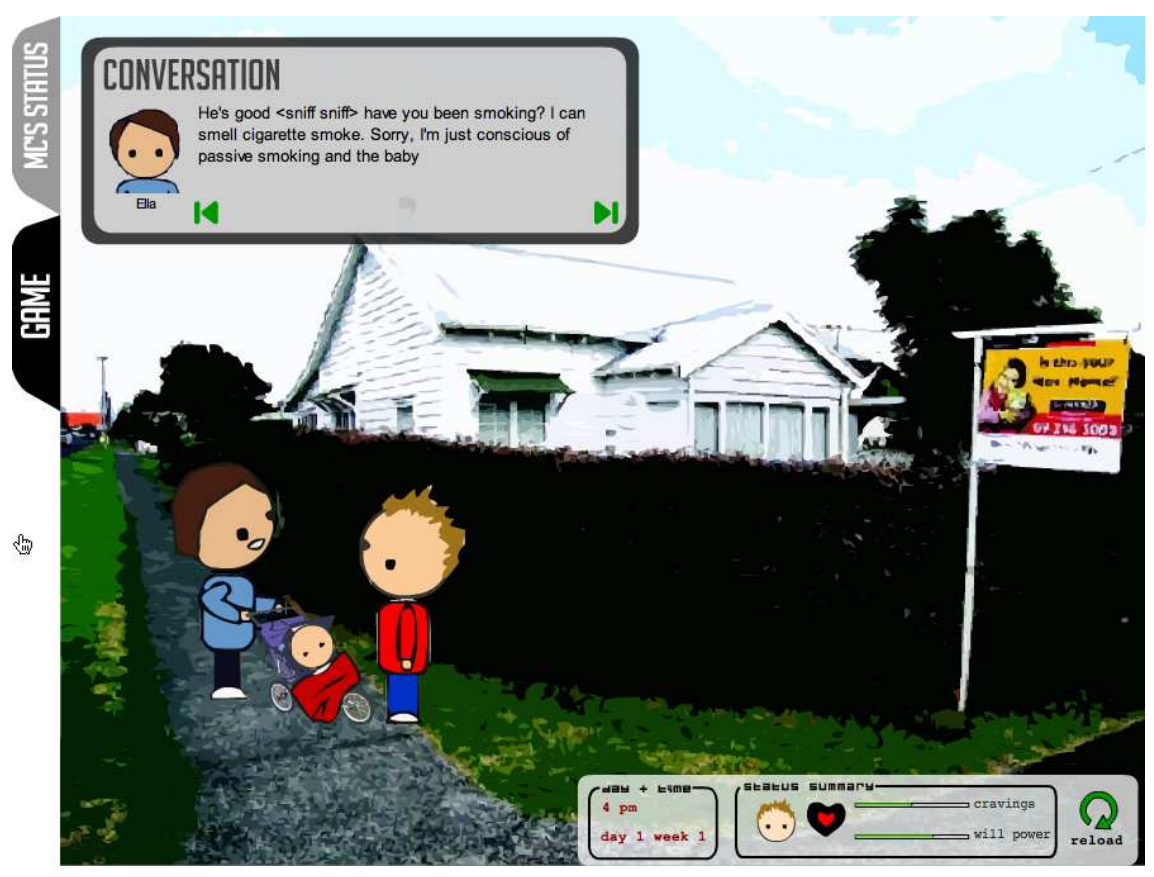

Figure 6.2: A chance encounter between MC and a family friend

et al. showed that in an American population of smokers trying to quit, low selfefficacy was a consistent predictor of relapse behaviour [60]. Self-efficacy is an important concept in Western psychology [15], but it seemed overly technical to reference in the game. Instead, we incorporated the related, but less technical concept of self-esteem. In upcoming $\$ 6.3 .2 .1$, we discuss how we did this.

A further way in which the NZE version of Smoke? references self-control is through the nature of the contexts in which MC demonstrates well-being benefits gained from quitting smoking. In the minigames, $\mathrm{MC}$ is essentially carrying out a solo activity. Figure 6.3 shows a screenshot from the NZE fitness minigame. We discuss our reasoning behind the solo minigame settings in further detail in $\S 6.3 .2 .1$.

\subsubsection{Personally-relevant future goals}

"You could insert your own incentive" In the NZ European focus group, participants expressed the importance of not assuming that everyone shares the same goals for the future. To foster a closer relationship for players with $\mathrm{MC}$, we decided that players should be able to customise certain aspects of MC in both versions of the game. The opening sequence of both versions of Smoke? asks players to specify a gender for $\mathrm{MC}$ and potential future careers. The gender choice determines the gender of $\mathrm{MC}^{\prime}$ s best friend and love interest. In this prototype of Smoke?, the gender of the best friend is matched to $\mathrm{MC}^{\prime}$ s gender, and the gender of $\mathrm{MC}^{\prime} \mathrm{s}$ love interest is the opposite gender. The career choices impact on the 


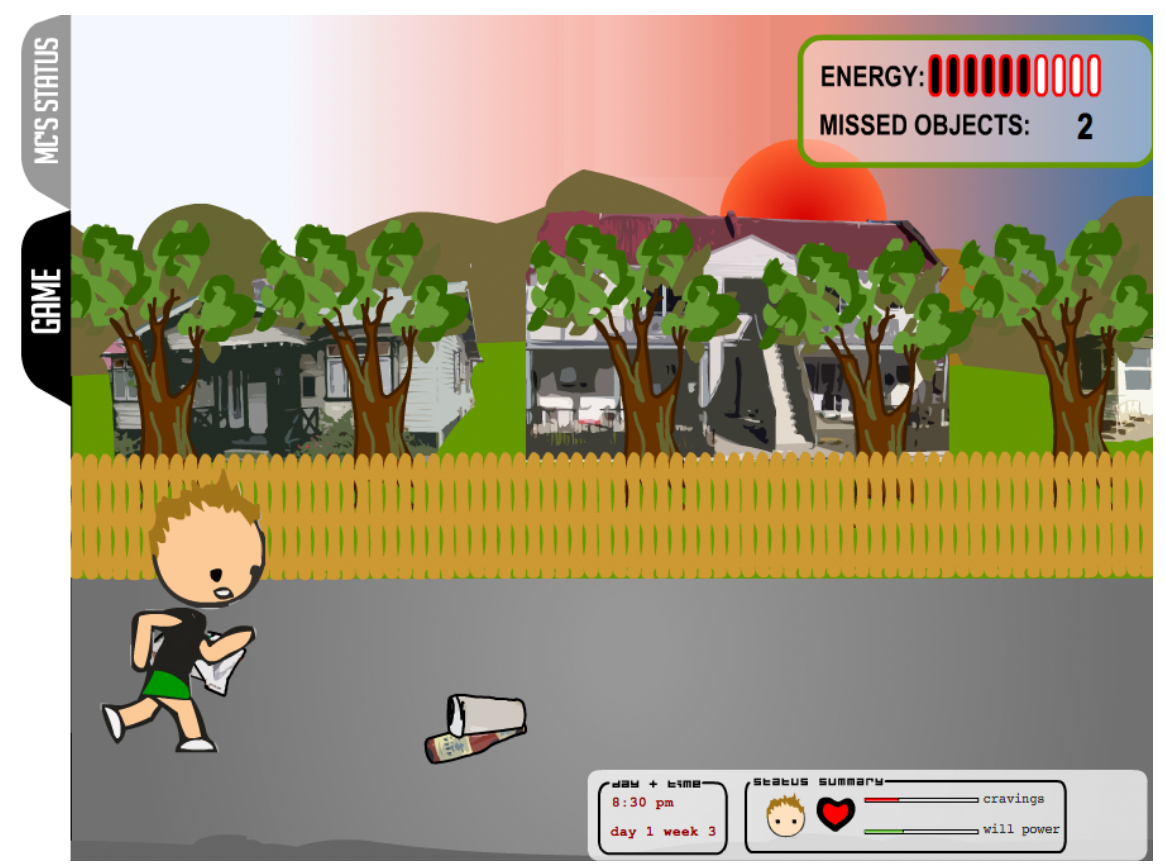

Figure 6.3: The fitness-running minigame

types of craving control activities offered to players during the game. For example, choosing to make MC aspire to be an athlete yields different activities from making MC aspire to be a trend-spotter. In the NZE version of Smoke?, in particular, the career path is again referenced at the end of each play-through of the game. The intention is to place smoking and its consequences in the context of a personally-relevant future life. Figure 6.4 shows a screenshot from this outcome sequence, referring to $M C^{\prime}$ 's future life goals.

In addition, the NZ European focus group members viewed incentives and personal financial savings as a powerful motivation for quitting smoking. While we allude to monetary savings in the contents of both versions of Smoke? (e.g. figure 6.5 shows a screen featured in the two versions), in the NZE version, we placed more emphasis on MC's potential personal savings achieved by not buying cigarettes. We discuss the details of how we did this in §6.3.2.3.

\subsubsection{Insights into design: the NZM version}

\subsubsection{Relevancy cues}

"Because I'm Māori there's a higher chance that they're gonna affect me" In the NZ Māori focus group, participants pointed out that their identity as Māori served as a self-relevancy cue for paying attention to many social marketing messages. To foster a sense of cultural identification between Māori players and the NZM version of Smoke?, we decided to design some of the contents to reference 


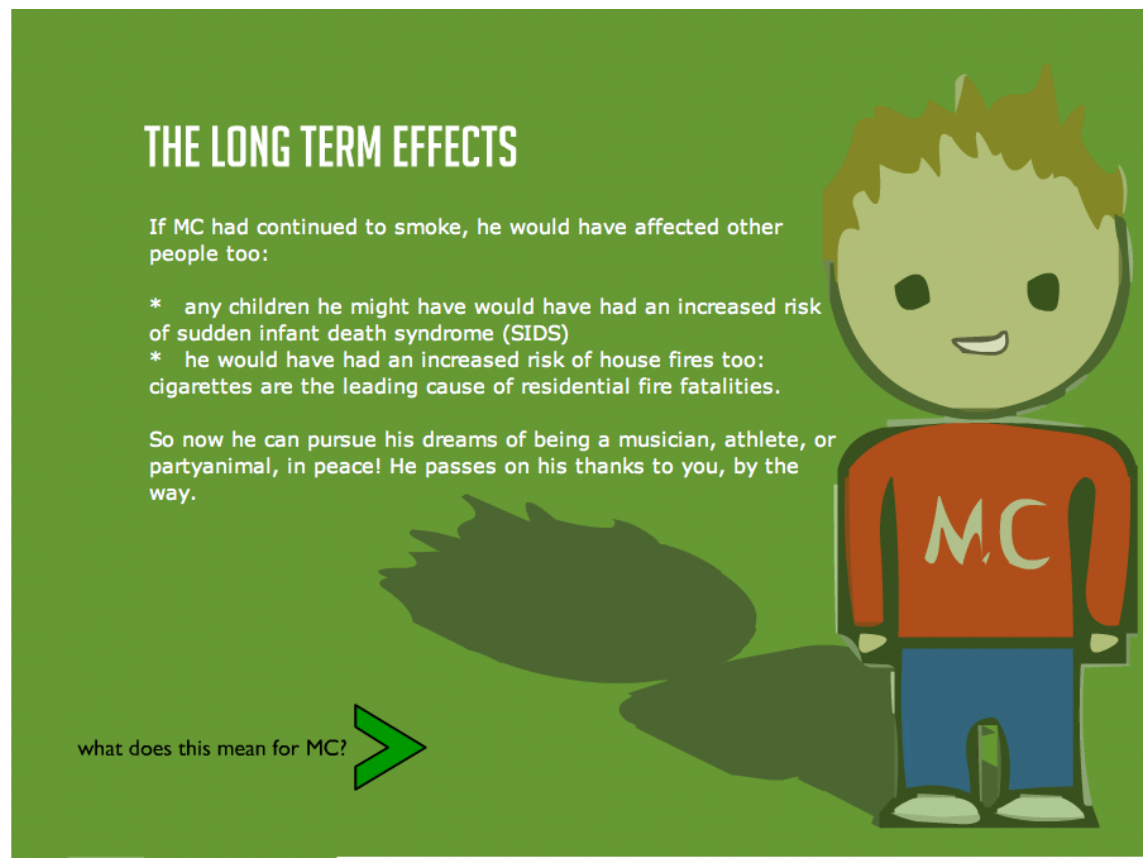

Figure 6.4: References to future plans in the outcome sequence

Over weeks three \& four, your influence over $\mathrm{MC}$ has resulted in the following:

By not spending money on cigarettes over the last couple of weeks, MC has saved another $\$ 66.00$. So that is a potential savings of $\$ 132.00$ in total over the last month. You can save last fortnight's money in the piggy bank again.
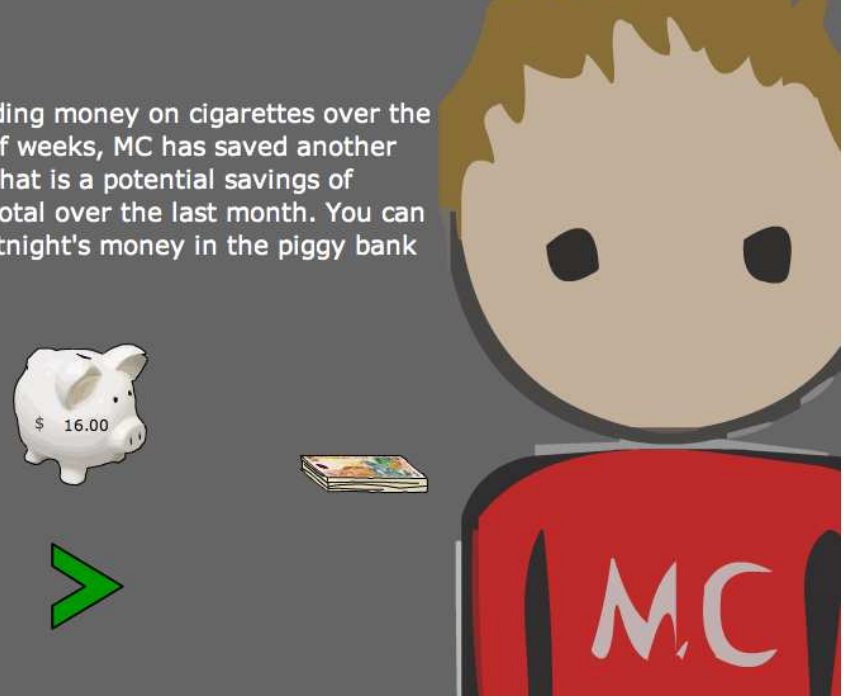

Figure 6.5: Emphasising monetary savings 
Māori culture both visually and contextually. We were wary, however, of unintentionally introducing negative stereotypes about Māori into the game. To avoid doing this, we engaged in a process of consultation with members of the Toihuarewa, to determine suitable cultural references. In accordance with their suggestions and approval, the NZM version contains characters with a range of skin tones, references typical rituals (such as spontaneous visits by family members and extended shared family meals), certain cultural values (for example, looking after and being looked up to by younger family members and respecting family elders), and a small amount of Māori language.

We were aware, however, that in referencing overt aspects of Māori culture, any attitude shifts and responses from players would at least partially be a result of reactions to the overt cultural cues. An alternative option would have been to completely avoid all overt Māori cultural cues. We decided against this, however, as we wanted the real world utility of the game content to be obvious for persuasive learning purposes. To partially balance the overt Māori cultural cues in the NZM version of Smoke?, we also introduced characters with a range of skin tones, and small amounts of Māori language in the NZE version.

\subsubsection{Role modelling}

"It's so hard to figure out why I'm a role model but I know I am one" The awareness of being a role model was a strong theme that emerged from the Māori focus group discussion. All participants emphasised the importance of passing on values to younger family members. More generally, in Māori culture, as in other collectivist cultures, there is an expectation that all members of a family will contribute to family activity $[51,69,143,162]$. To introduce the role model concept into the NZM version of Smoke? we included a character who specifically views MC as a role model. This is a little sister character, named Jade. To emphasise the importance and persistence of role modelling, Jade is almost always around MC, looks up to $\mathrm{MC}$, and wants to spend time with $\mathrm{MC}$, despite the age difference between them. The dialogue of the NZM version features conversations between MC and Jade in which Jade asks why MC smoked, whether the negative effects of smoking will affect other family members, as in figure 6.6, and why MC decided to quit.

To reinforce the idea that MC's actions affect his sister's beliefs, the conclusion of the game features a prediction about the likelihood of her smoking, and suffering from smoking-related illnesses. We discuss the details of the incorporation of the MC-Jade role model concept further in §6.3.3.3.

The role model concept, however, has two sides. In Māori culture, people 


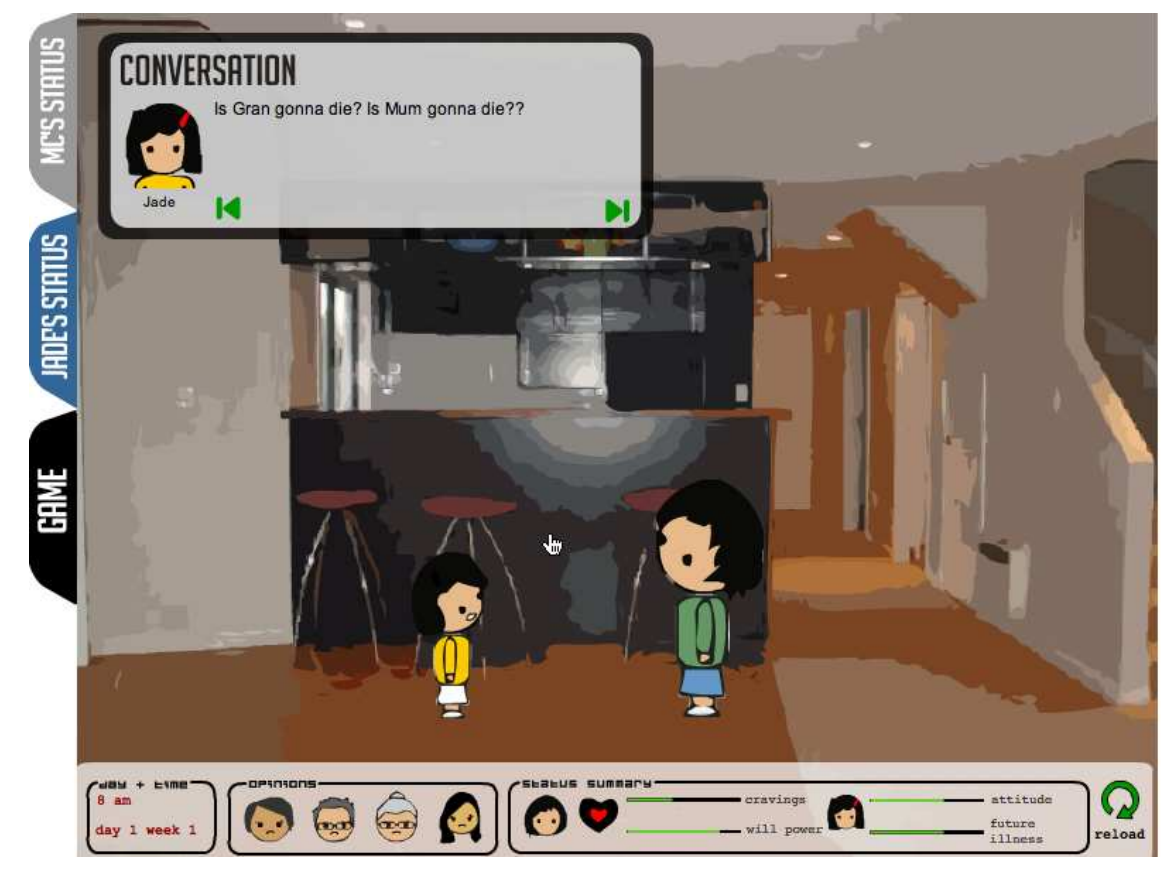

Figure 6.6: Jade asks MC a sensitive question

act as role models for younger family members, and also look to older family members as role models. The NZM version contains a number of interactions between MC and elder family members, who are role models. See $\S 6.3 .3 .1$ for further discussion on how elder characters influence MC in the NZM version of Smoke?

\subsubsection{Fitting in}

"When I first started smoking, it was because so many of my friends do" "Coolness" was explicitly noted as not being a motivation for members of the Māori focus group. While the perception for what constitutes "coolness" is largely subjective, a consistent reason given for starting to smoke expressed by all the smokers in the Māori focus group was peer group endorsement. We considered it vital for the narrative of the NZM version of Smoke? to include influential friend characters who were smokers. In the NZM version, then, the majority of MC's old friends, including MC's best friend, remain smokers throughout the game.

A typical issue faced by ex-smokers is the redefinition of their relationships and interactions with smokers, especially if they are family members or close friends. We decided that the redefinition of behaviour around smokers should be addressed in the narrative of the two versions of Smoke? In one branch of both game versions, $\mathrm{MC}$ encounters a situation in which his quitting alienates some of his friends, to the point where they have begun to avoid him, as displayed in figure 6.7. In the NZM version of Smoke?, however, we wanted to emphasise 


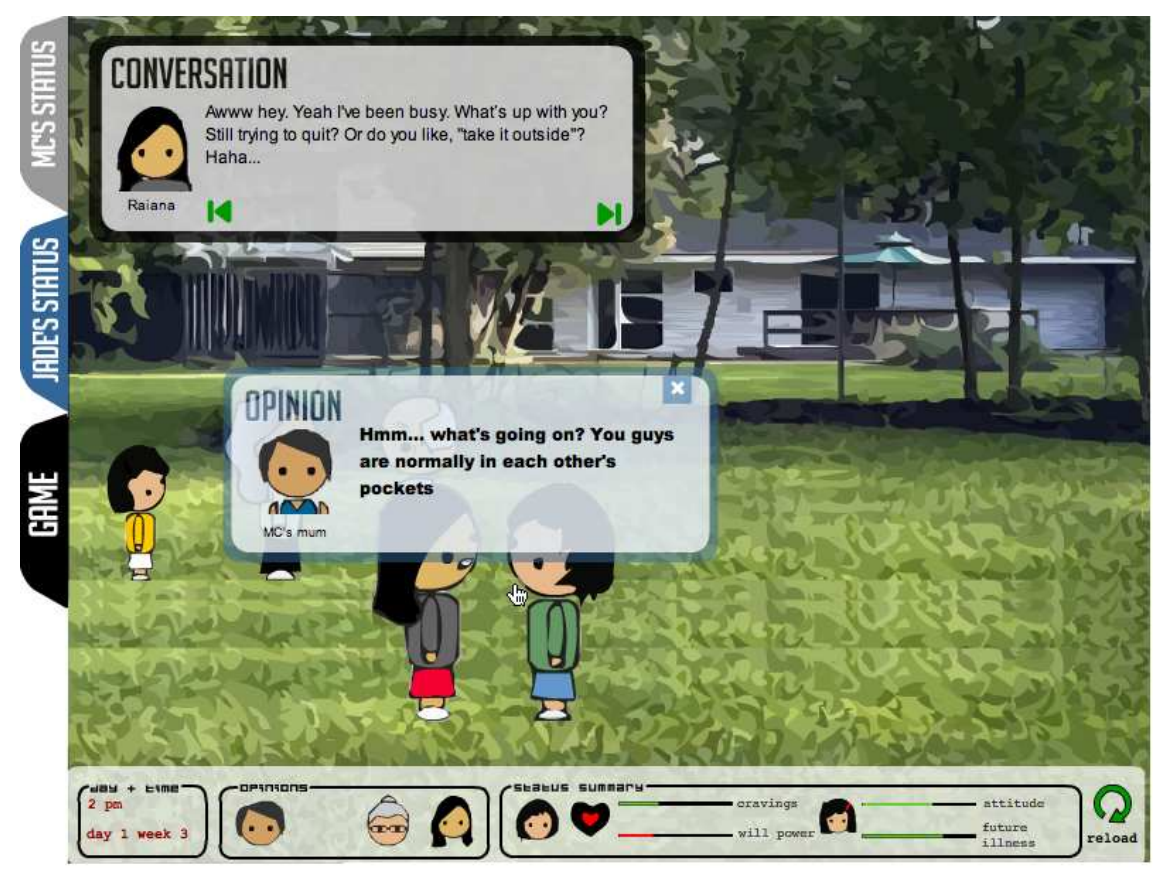

Figure 6.7: $\mathrm{MC}^{\prime} \mathrm{s}$ best friend explaining her recent absence

the powerful effects of peer pressure, and the social alienation caused by quitting. To communicate this, we decided that MC's best friend in the NZM version of Smoke? should also be his cousin. This increased the number of opportunities for MC to encounter his best friend as there is much interaction between family members in collectivist cultures.

Another way in which we alluded to the themes of peer pressure, influence, and attitude formation, was through the introduction of an additional status screen to the NZM version, reflecting Jade's attitudes towards smoking, and her future likelihood of contracting a smoking-related illness. In the game, MC's actions are the only precursors for Jade's attitudes: if he is living a smoke-free lifestyle around Jade, it is reflected in her status screen. We discuss Jade's status screen further in $\S 6.3 .3 .3$.

\subsubsection{An acceptable habit}

"With smoking, in my family, it's a given" Acceptability of smoking as a habit was an additional reason for starting to smoke cited by members of the Māori focus group. To reflect the acceptance of smoking, in the NZM version of Smoke?, $M C^{\prime}$ s mother and grandmother are both smokers. A common grave health consequence linked with smoking is lung cancer. In the narrative, $\mathrm{MC}^{\prime}$ 's grandmother suffers from this disease. After consultation with Toihuarewa members, we decided to set one plot development scene of the NZM version in the hospital room of the grandmother. A member of the Māori focus group described a similar experi- 
ence of her own, where she left the hospital feeling convinced that she needed to quit.

While smoker-friendly social environments are powerful, Māori focus group members also recounted experiences of encountering people they respected who strongly (but positively) encouraged smoke-free lifestyles. The NZM version of Smoke? features an aunt character who is an active advocate of smoke-free lifestyle. She strongly discourages people smoking in public and at her house. It was important to include a positive, smoke-free viewpoint endorsed by a Māori game character, to counter the stereotype of Māori as smokers.

\subsubsection{Personal reward}

"You've got so many responsibilities as it is that you want something for yourself" For the most part, the Māori group members viewed smoking as an activity that had consequences reaching beyond the individual. A couple of the smokers in the group, however, pointed out that they viewed smoking as a personal reward for shouldering collective responsibilities. Referencing this idea in both versions of the game, and to remind players that there are other ways to reward themselves, players can adopt at least one luxury reward activity. Players may choose to use the luxury activity later on to manage MC's cravings. Additionally, both versions of the game also feature one craving control activity that can be collected only by doing well in the fitness minigame.

\subsubsection{Social environment}

"There's always someone around who's got a cigarette" The Māori group members talked about how cigarettes were easy to obtain, because smokers were almost always present in their social environment. We drew from this the implication that they were accustomed to being surrounded by others, and some of them would be smokers. In response, the minigame scenarios of the NZM version of Smoke? present MC and his quitting struggles in the social context of his friends and family. We further discuss our reasoning for the NZM minigame contexts in $\S 6.3 .3 .2$.

Another implication of the Māori group members claiming that cigarettes were readily available from others, was that cessation arguments based on financial motivations were likely to be less effective on Māori players. For this reason, we treated financial incentives differently in the two versions of the game. We discuss this further in $\S 6.3 .2 .3$. 


\subsubsection{Advice from younger family members}

"If the kids would ask the adults, that would make a difference to me" Members of the Māori focus group expressed their resistance to pleas for smoking cessation from older family members, when many of these family members remained smokers themselves. They did, however, claim that they would be willing to heed advice from non-smokers in the family.

In the NZM version of Smoke?, Jade partially serves this role. She is almost always present, and during the game, she is a non-smoker. Both versions of the Smoke? game narratives also feature a number of other positive role model nonsmoker characters, two of whom are approximately $\mathrm{MC}^{\prime}$ 's age.

In a related, but more active vein, game persuasion typically concerns persuading game players, but we decided to add an additional layer of persuasion to the NZM game, in the form of an extra minigame, in which the goal for players was to persuade a game character. Specifically, this minigame calls on players to get $\mathrm{MC}$ to convince his best friend to quit, by formulating the correct combination of cessation arguments that will appeal to him. Persuasion studies have shown that the process of generating and formulating arguments favouring one side of an issue often persuades people that this is the more defensible position, regardless of the side they were allocated to defend [114]. We discuss the challenge further in $\S 6.3 .3 .4$.

\subsection{Incorporating the culturally-relevant PT design strategies into Smoke?}

\subsubsection{Decision and incorporation process}

After exploring ways in which we could use the focus group insights, our next step was to determine how we could incorporate the PT design strategies into the two versions of the game. In determining whether or not we could use a particular strategy in our game designs, we took into consideration various aspects of each strategy:

- how the strategy could support the focus group findings.

- how the strategy could be implemented in simulation/adventure games.

- how the strategy was persuasive with regards to learning about quitting smoking. 
- at what level of the game the strategy could be implemented, i.e. a feedback level, a narrative level, or a basic game functionality level.

- whether the dissemination format (i.e. web-based but standalone) supported the realisation of the strategy.

- whether the Flash development platform could support certain implementations of the strategy.

- whether the strategy could be implemented in the given timeframe.

Through an iterative process of design brainstorming and referring to the focus group data, we established a plan for incorporating several strategies into the separate versions of the game. It was not feasible to incorporate all the strategies into the designs, largely due to the small scope of our project, coupled with the fact that our PT prototype was a game. Here we discuss how we harnessed the strategies within the NZE and NZM versions of Smoke? We focus more on differences between the two versions of the game, rather than their similarities.

\subsubsection{Tactics into design: the NZE version}

\subsubsection{Using the SELF MONITORING strategy}

The SELF MONITORING strategy allows people to monitor themselves, for the purpose of learning how they might modify their attitudes or behaviours to achieve a pre-determined goal or outcome. Both the NZE and NZM versions of Smoke? feature status screens relating to $\mathrm{MC}^{\prime}$ 's well-being. These can be viewed as applications of SELF MONITORING. They reflect how players are making MC act and indicate how they can improve MC's well-being. In $\S 4.2$ we discussed how SELF MONITORING is more suited to individualist, rather than collectivist audiences, and our NZ European focus group members had talked about how they believed that quitting smoking was in the control of the smoker. We decided, therefore, that SELF MONITORING should play a more obvious role in the NZE version of Smoke?, than in the NZM version.

SELF MONITORING application: the self-esteem score In the design of the NZE version of Smoke? it was important to guide players' views of MC to be, if not extensions of themselves, at least someone they felt in control of, and responsible for. We thought that this might motivate players to make the types of choices for $\mathrm{MC}$ that they would for themselves, and as a result, consider the game contents in the context of their own lives. Self-esteem is a concept that is familiar to individualists, and commonly referenced in Western psychology [38, 96]. To increase the 
Figure 6.8: The self-esteem score from the status screen

possibility of players identifying with MC, the NZE version of Smoke? features a self-esteem score alongside the other variables monitored for MC in the status screen. Given that self-esteem usually refers to an individual's self perceptions, we thought its presence as a score would strengthen players' identification with and responsibility for $\mathrm{MC}$, and consequently, their drive for self improvement as mediated through MC.

A study of quitting behaviour showed that people's perceptions of their quitting ability are predictors of their relapse behaviour [60]. This research seems to support the idea that self-esteem is a shaping factor in a smoker's perception of his/her ability to quit. The self-esteem rating in Smoke? is entirely contingent on how players make MC behave and a low rating might trigger a nicotine craving. The act of smoking also results in a drop in self-esteem, reflecting a decrease in $M C^{\prime}$ 's belief that he is capable of quitting. On the other hand, if players make MC cope by carrying out a craving control activity, MC benefits from a rise in self-esteem. This interaction is supported by anecdotal evidence cited by smoking cessation experts we interviewed.

SELF MONITORING application: solo minigames Another perspective on the SELF MONITORING strategy is that it emphasises the personal nature of causeand-effect relationships resulting from adopting particular courses of action. The positive consequences of smoking cessation are straightforward in terms of personal benefits, especially health benefits. To emphasise a personal locus of control, then, both the NZE minigames concerning health and well-being feature solo challenges, where MC is largely the only character present. The fitness game features $\mathrm{MC}$ on a solo running mission (see figure 6.3), while in the concentration game, $\mathrm{MC}$ is shopping by himself at a supermarket (see figure 6.9). By removing other influential social actors from the challenges, we wanted to focus players' attention on $\mathrm{MC}^{\prime}$ s personal progress, and reduce associating progress with motivations such as competition, or obligation, which can surface in more social environments

\subsubsection{Using the TUNNELLING strategy}

The TUNNELLING strategy reduces complexity and serves to lower uncertainty, by leading users through a predetermined sequence of actions or events, step by 


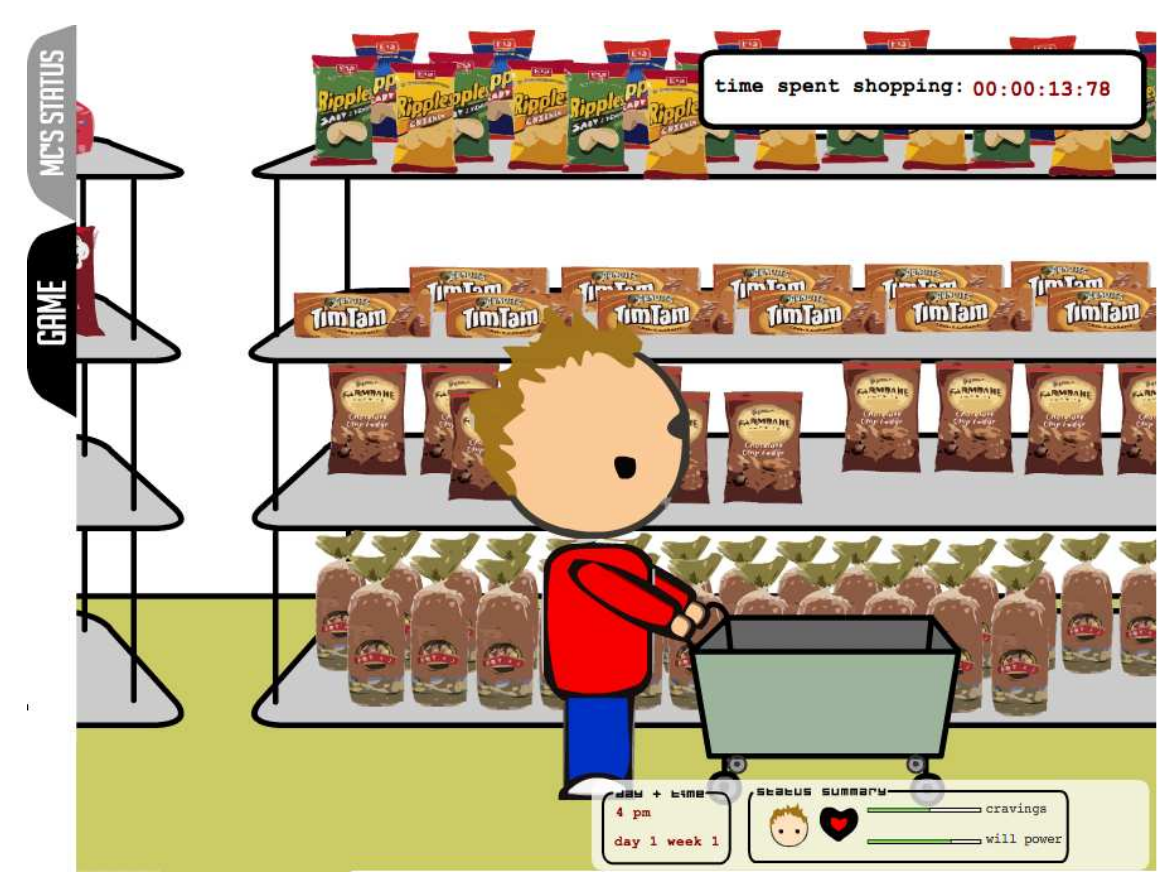

Figure 6.9: The concentration-shopping minigame

step, to encourage certain behaviours. In $\S 4.2$, we discussed how TUNNELLING was more suited to individualist, rather than collectivist audiences. Given that members of the NZ European focus group had talked about how smoking was under a personal locus of control, we thought that this strategy could be used within the game to highlight personal control.

TUNNELLING application: autonomy focus Early in the NZE version of Smoke? a number of NPCs are introduced in the narrative. As players progress through the game, however, these NPCs will not necessarily reappear in MC's life. Unless players attempt to have MC maintain a relationship with any particular NPC, or rely on the NPC's support, the influence of the NPC is gradually removed. By emphasising MC's autonomy, we hoped to familiarise players to the idea that they alone could make $\mathrm{MC}$ reach a quit state and that this success was not dependent on the actions of other NPCs.

\subsubsection{Using the ACHIEVEMENT strategy}

The ACHIEVEMENT strategy concerns showing users outcomes or consequences resulting from their performance of goal-related target behaviours. It is similar to the TAILORING strategy, but focuses on longer term outcomes, and showcases to others what users have achieved. The NZ European participants talked about how they felt that quitting incentives were more effective if they were customised. One technique based on TAILORING used by several smoking cessation websites 


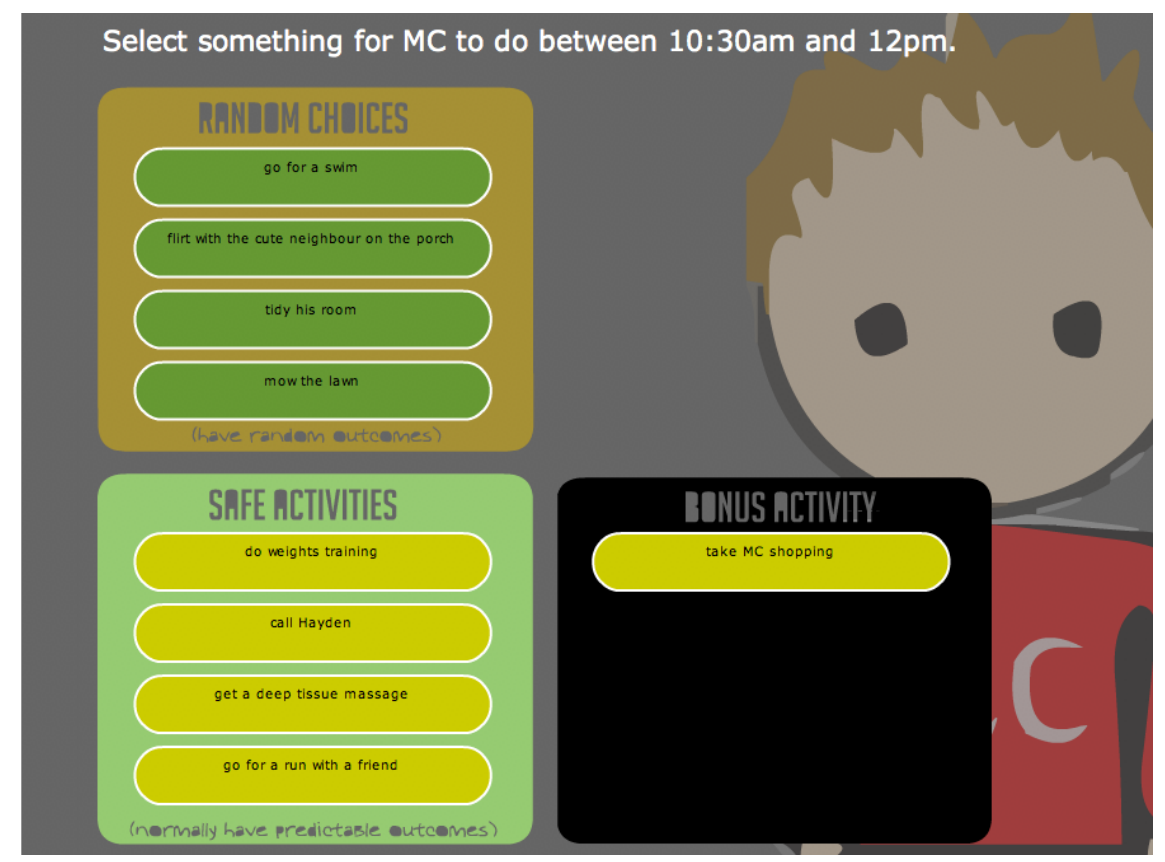

Figure 6.10: The shopping with savings option

is the savings calculator (e.g. that provided by QuitNet [65]). Typically, the savings calculator represents items that smokers can obtain by not spending money on cigarettes. In our focus groups, the NZ European participants talked about how they believed financial incentives were strong quitting motivations. In contrast, the Māori participants believed that smoking was less directly associated with cost, as cigarettes were always available when needed.

ACHIEVEMENT application: shopping In the NZE version of Smoke?, if players succeed in not letting MC lapse during plot development scenes, they can save money that would have otherwise been spent on cigarettes. Later, during wildcard scenes, players have the option of taking MC shopping with the money they have saved, as in figures 6.10 and 6.11. Over the course of repeated shopping trips, funded by these savings, players can customise and upgrade MC's appearance, with new clothes and accessories. At the start of the game, MC's clothes and appearance are quite similar to those of other NPCs, so we hoped that the possibility of gradually differentiating MC from other NPCs would motivate players to continue to keep MC smoke-free.

\subsubsection{Using the CONDITIONING strategy}

The CONDITIONING strategy concerns the use of operant conditioning to reinforce target behaviours when they occur. In PT design, Fogg suggests that designers use positive, rather than negative reinforcement [46]. CONDITIONING using 


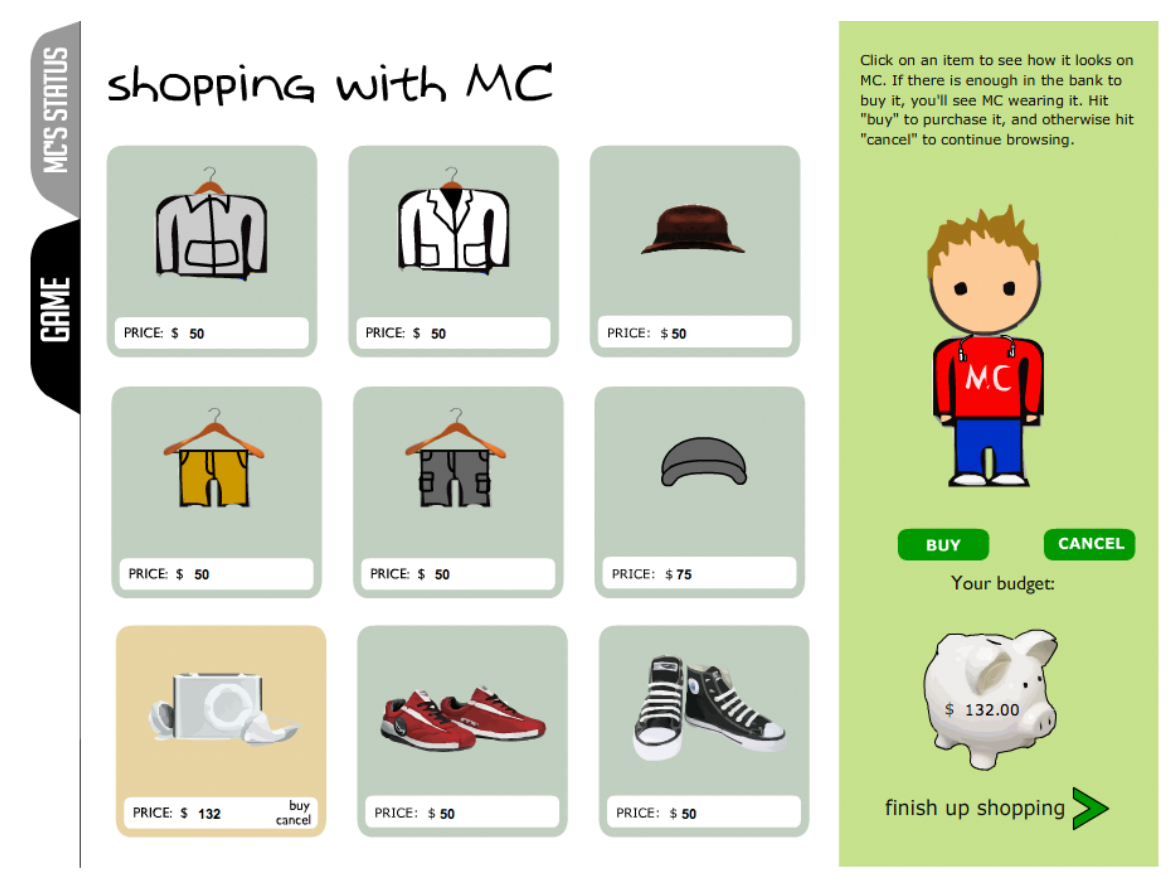

Figure 6.11: Shopping for an iPod

positive and negative reinforcement is a frequently used strategy in traditional game design, especially in the construction of rules, scores, and outcomes. Both versions of Smoke? feature status screens for MC and an overall quit state outcome. All of these can be construed as CONDITIONING.

CONDITIONING application: game feedback In section 4.2, we discussed how collectivist audiences are likely to be accustomed to focusing on undesirable behaviours. In fact, this reasoning partially motivates our DISESTABLISHING strategy. While in the NZE version of Smoke? we wanted to minimise the use of DISESTABLISHING, we still wanted the game to communicate that allowing MC to lapse would result in MC's failure to quit. Furthermore, when MC's overall state is low, it triggers cravings. This means that the game highlights undesirable situations, such as MC craving a cigarette, even when he has supposedly quit.

In the NZE version of Smoke?, however, we wanted players to encounter neutral and positive feedback, as opposed to negative feedback. Accordingly, the narrative of the NZE version of Smoke?, unlike the NZM version, features a minimal amount of DISESTABLISHING. It also features less overtly negative content related to smoking, apart from MC's state and the overall outcome, while including the same health information as the NZM version. 


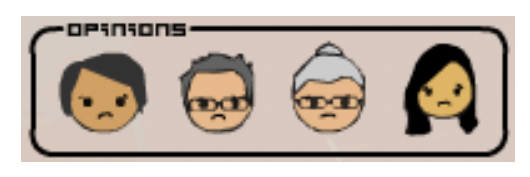

Figure 6.12: The moods + opinions box

\subsubsection{Tactics into design: the NZM version}

\subsubsection{Using the GROUP OPINION strategy}

The GROUP OPINION strategy involves providing users with the opinions of other ingroup members, or users similar to them, at moments when users are required to make important decisions related to their own goals. In our focus groups, we learned that the Māori participants were concerned about how their actions were being perceived by other family members. We decided to reference this notion within the NZM version of Smoke? in terms of MC's relations with his friends and family.

GROUP OPINION application: the moods + opinions box In the NZM version of Smoke? the moods + opinions box, shown in figure 6.12, contains iconic representations of three of MC's close family members, as well as his best friend. As players progress through the game narrative and interactions, each of the character icons' facial expressions may change in accordance with their current mood. Their mood is determined by how they individually perceive players' choices for MC. In addition to expressing moods, each character icon can express opinions on how MC is behaving. The addition of an opinion is indicated by the character icon flashing. These opinions typically appear when players are required to make a decision on behalf of MC. By clicking on a character icon, players can view that character's opinion about what MC should do. Figure 6.13 shows an example of the grandmother character sharing her opinion. The character icons do not have to be active NPCs in the game narrative at the time.

GROUP OPINION application: opinions from elders The NZM version of Smoke? features a number of characters who may be classified as elders. One example is the grandmother character who is a current smoker, and another is an aunt who is an active advocate of smoke-free lifestyles. Over the course of the game, depending on the narrative branches players encounter, MC may encounter both of these characters (see figure 6.14). Both the grandmother and the aunt share their opinions about why they believe it is important for MC to quit, and how MC should behave in relation to other characters. These opinions may guide players in upcoming decisions in which they choose courses of action for MC. In Māori culture, 


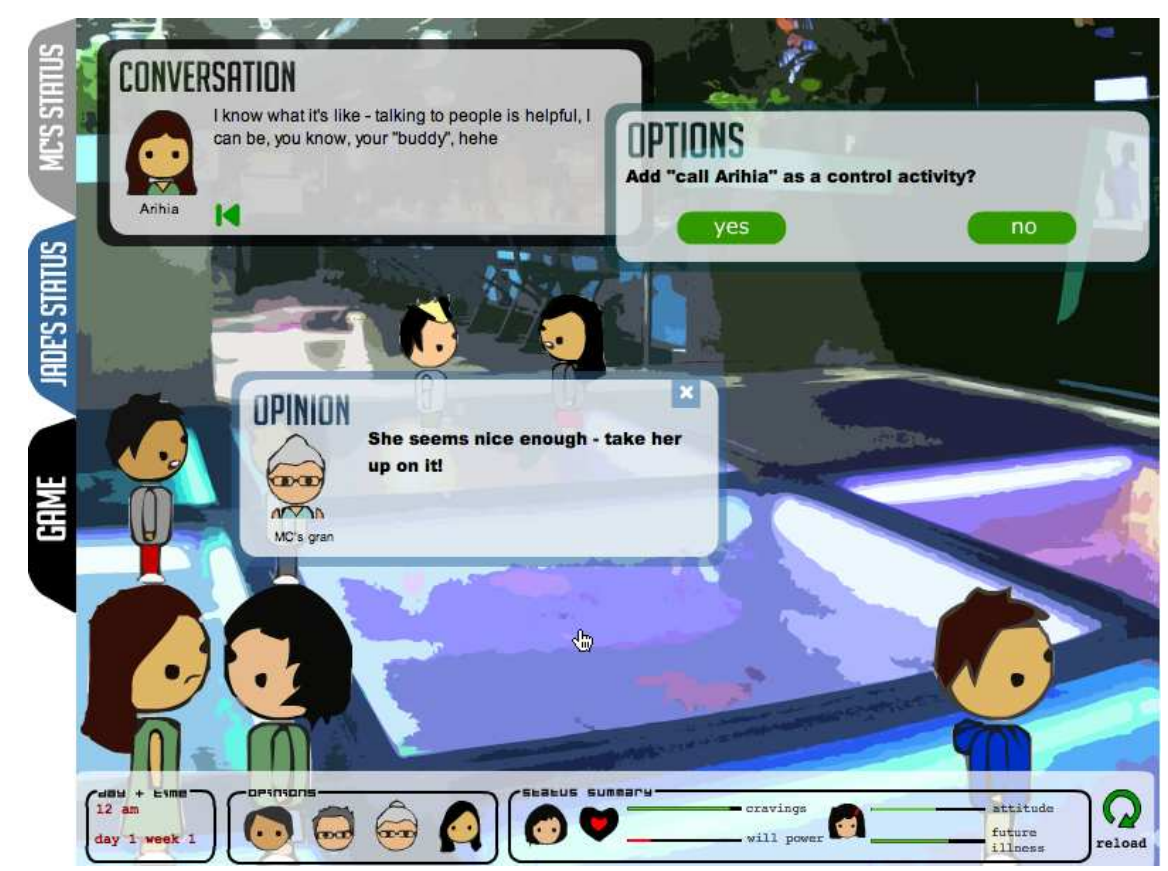

Figure 6.13: Gran's opinion of MC taking Arihia's phone number

and collectivist societies more generally, elders hold an esteemed position in society, and are often looked to as sources of advice $[69,96]$. In addition, research on anti-smoking socialisation shows that children are less likely to begin smoking when they come from households in which parents actively talk about not accepting smoking, even if the parents are smokers themselves [67]. The grandmother's stance is an application of this idea to an extended family context.

\subsubsection{Using the HARMONY strategy}

The HARMONY strategy involves the presentation of social density cues to users. The cues serve to suggest to users that they are in a socially dense environment with members of their ingroup in order to subtly promote harmonious actions that support the group's goals. In incorporating HARMONY in the game, we hoped that players would be triggered into making $\mathrm{MC}$ act in a way that supported the goal of cessation as an inherent goal of MC's family. We note that if smoking is considered as a harmonious activity, however, and the group in question is constituted by smokers, the strategy reinforces the effects of peer pressure, and promotes smoking. Research on group psychology indicates that as long as a group contains at least one dissenter who does not subscribe to the group norm, it is more likely that other members of the group will deviate from the group norm [83]. Thus, in peer pressure situations, where MC is pressured to smoke with his friends, there is always at least one other important NPC present or nearby, who is clearly a non-smoker, as in figure 6.15 . 


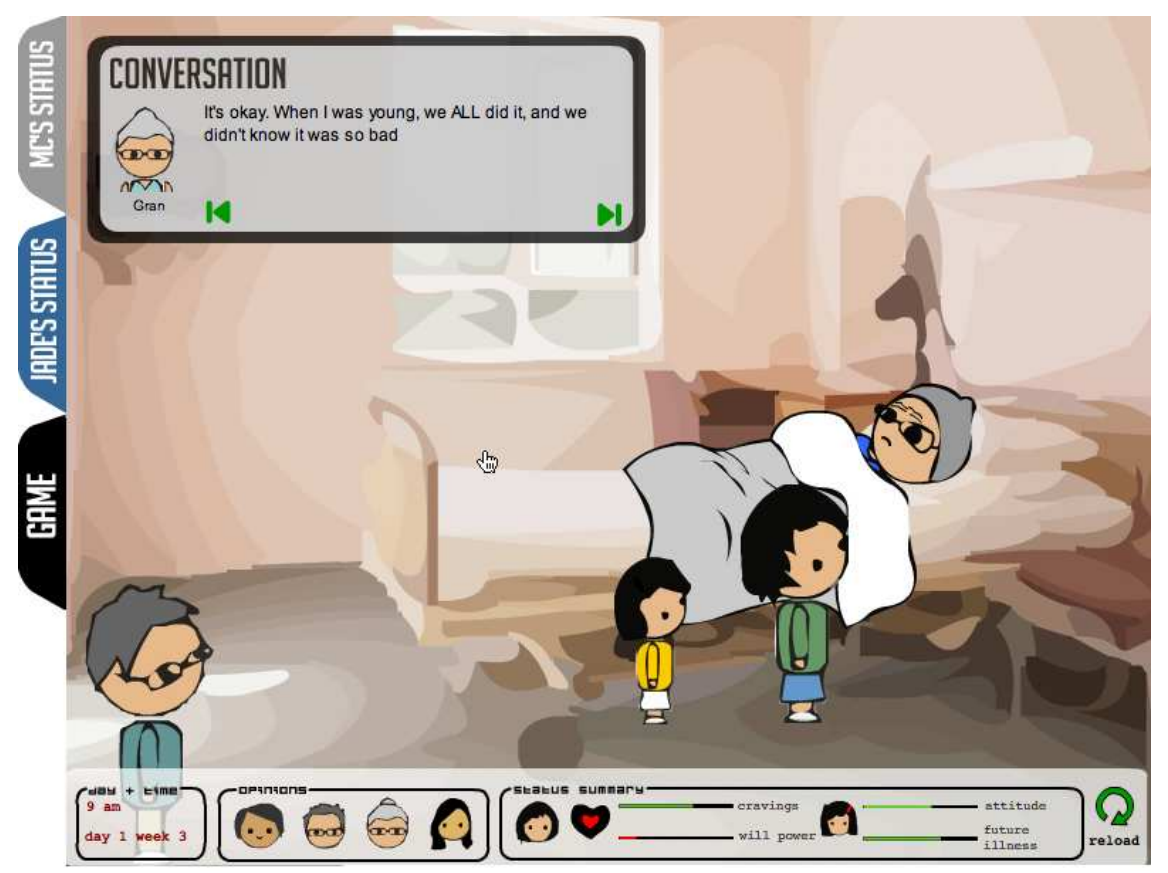

Figure 6.14: Gran in hospital talking to MC and Jade

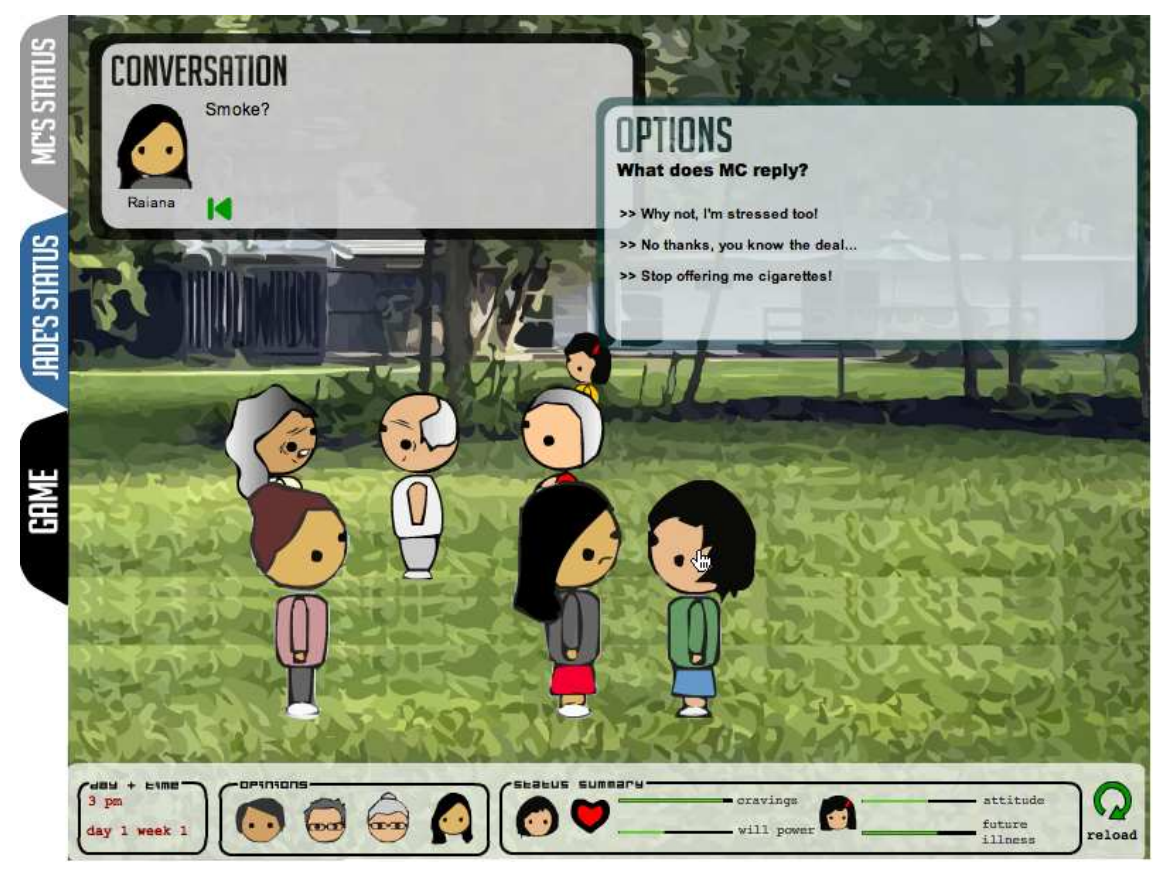

Figure 6.15: MC's best friend offers MC a smoke 
HARMONY application: general character density Scenes of the NZM version of Smoke? contain numerous references to NPCs: the dialogue refers to many other characters, the moods + opinions box is always visible, and every plot development scene always features at least one other NPC (e.g. see figures 6.13, 6.14, 6.15, etc.) By contextualising smoking-related issues in socially dense environments, we hoped that players would be triggered to consider the effects of passive smoking on others, serving as a role model for other family members, and the effects of peer pressure.

HARMONY application: moods + opinions box The moods + opinions box, shown in figure 6.12, presents players with the moods and opinions of MC's family members and best friend. While players are under no obligation to make MC act according to the wishes of the characters referenced in the box, or even consult their opinions, the moods and opinions have been designed to be generally supportive of MC's quitting objective. If players want to keep the moods + opinions box characters happy, then they have additional motivation to keep MC smoke-free and maintain harmony with these characters.

HARMONY application: social minigames As mentioned earlier, the two versions of Smoke? feature different minigames. While the NZE minigames are designed to draw attention to MC in an individual context, the NZM minigames are designed to highlight MC's progress (or lack of process) in a highly visible social context. This context is peopled with others he cares about and whose opinions matter to him. The fitness minigame is a modified version of tag in which MC's cousins have been entrusted in his care. The concentration minigame, depicted in figure 6.16, requires the player to help MC make sandwiches for other family members, who watch and react to MC. In both of these scenarios, we intended players to feel motivated by their responsibility towards other members of MC's ingroup ${ }^{4}$.

\subsubsection{Using the MENTORING strategy}

The MENTORING strategy involves tracking behaviour that users wish to change, in a similar way to the SURVEILLANCE strategy, but making the information available to other group members. The group members then act as mentors and help to keep users motivated to change their behaviour. Participants from the Māori focus group talked about the impact that other people's perceptions had on their

\footnotetext{
${ }^{4}$ Both of these scenarios were directly inspired by comments from participants in the Māori focus group.
} 


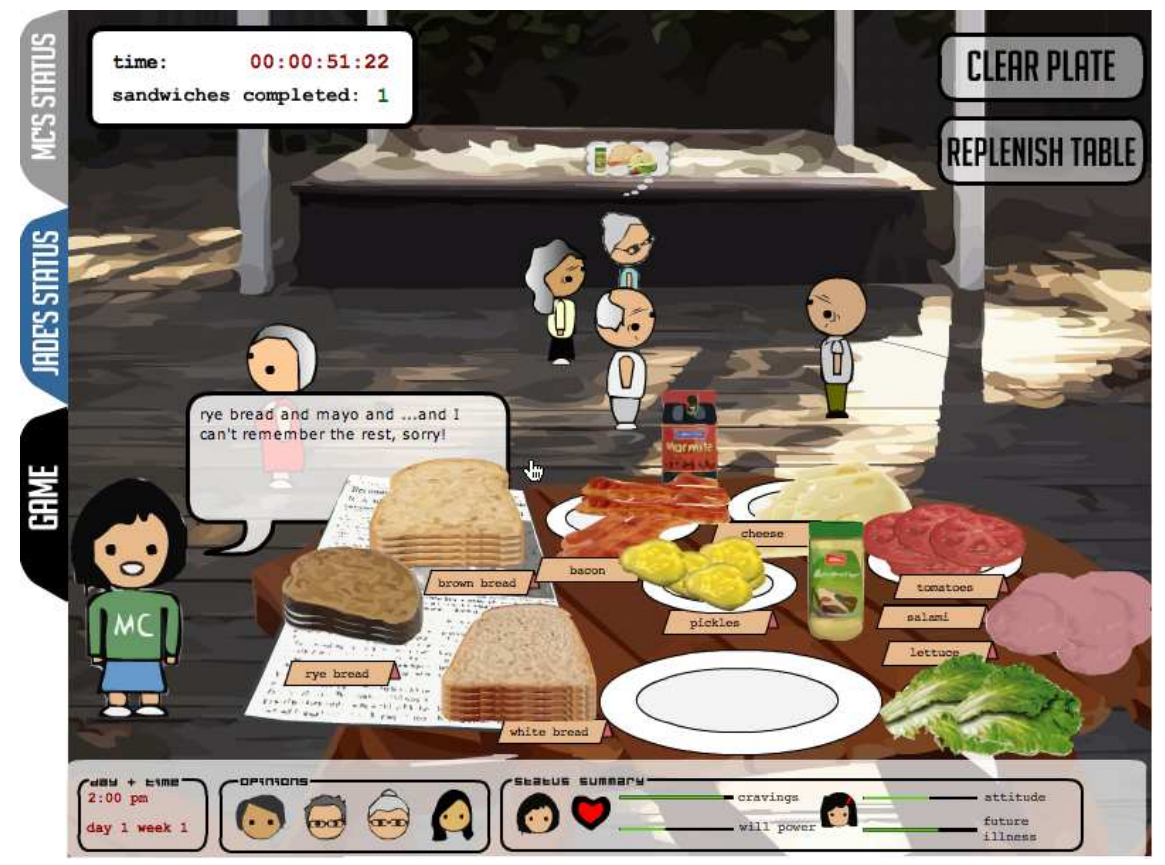

Figure 6.16: The concentration-sandwich minigame

behaviour. They also emphasised the importance of acting responsibly as role models for younger family members, and commented that they wished other family members had not smoked in their presence.

MENTORING application: Jade status As mentioned earlier, the NZM version of Smoke? prominently features one character that the NZE version does not: Jade, the younger sister. Jade spends a significant amount of time with MC, and looks up to him as a role model. Jade has her own status screen which details the likelihood of her being adversely affected by smoking in the future. Jade's status is directly related to MC's actions because what MC does in Jade's company is reflected in Jade's future attitudes and smoking likelihood. While this is an overly simplified cause-and-effect relationship, we anticipated that awareness of being a role model or a mentor would trigger players into making choices that seemed responsible.

MENTORING application: the respect score A fundamental value in Māori culture is respect: all things have a mauri, or life force, that must be respected. If mauri is not respected, it loses its vitality and will not flourish [109]. In place of self-esteem used in the NZE version of Smoke?, we decided to include a respect score in the NZM version (see figure 6.17). The respect score relates to how members of MC's family respect $\mathrm{MC}$ over the course of the game. It forms a part of MC's overall state and thus impacts on his craving level. In this way, we were 
Whanau respect

for MC:

\section{$\because$}

Figure 6.17: The respect score from MC's status screen

modelling an explicit link between other characters' perceptions of $\mathrm{MC}$, and $\mathrm{MC}^{\prime} \mathrm{s}$ state. We thought that by helping players to see how MC's actions were being perceived by others, they would be motivated to act in ways that would gain optimal levels of respect from other characters.

\subsubsection{Using the TEAM PERFORMANCE strategy}

The TEAM PERFORMANCE strategy concerns tracking the behaviour of individuals forming a group. It rewards or reprimands all members of the group on the basis of the actions of each individual which are related to a group behavioural change goal. Families in collectivist cultures are likely to have a strong opinion on the topics of family responsibility and acceptable behaviour, because family members feel so linked to one another, that they feel they stand to gain and lose based on each other's actions.

TEAM PERFORMANCE application: the convince best friend challenge In the NZM version of Smoke?, if players are largely successful in keeping MC smokefree, they are presented with an additional challenge towards the end of the game. The challenge is to convince MC's best friend to consider quitting smoking, by selecting four cessation motivation arguments that appeal to the best friend. Figure 6.18 shows a screenshot from this challenge. In a real world scenario, we are aware that the success or failure of MC convincing the best friend to quit would in fact have the greatest impact on the best friend, but in a collectivist culture, other ingroup members would also share in the best friend's successes and failures, as they would impact on the group overall [69, 96, 138, 143]. Highlighting the spirit of group solidarity, if players succeed in getting MC to present a successful combination of cessation arguments to the best friend, the best friend will consider quitting smoking. As a result, MC will benefit from a boost in mental well-being, reflecting a connection between his well-being, and the best friend's well-being.

\subsubsection{Using the DISESTABLISHING strategy}

The DISESTABLISHING strategy concerns training users out of practising specific actions or behaviours that they do not want to perform. It is similar to the CON- 


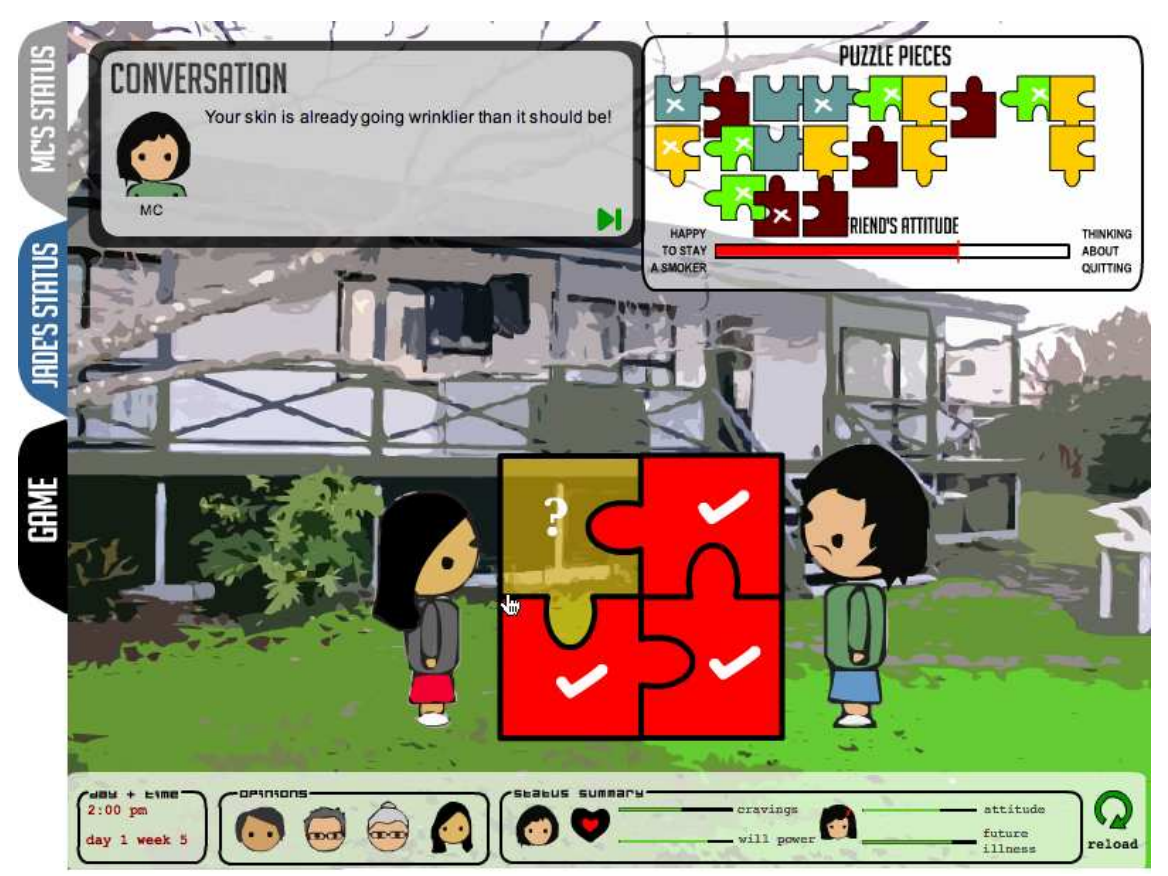

Figure 6.18: Convincing the best friend to quit

DITIONING strategy, except that it focuses on the elimination of undesirable behaviours by triggering approved reminder cues when the undesirable behaviour is detected. Our Māori focus group members talked about how they were aware of what not to do, because other family members pointed out their inappropriate behaviour.

DISESTABLISHING application: telling off MC's best friend Research on smoking cessation campaigns has shown that smokers often feel targeted by messages focusing on negative consequences, and begin to use disengagement beliefs to distort the messages [85]. We were wary of making players feel they were being explicitly reprimanded. To avert this reaction, the NZM game features an auntie character who is an advocate for smoke-free lifestyles, and critical of other smokers in the NZM game, but does not overtly direct her criticism at MC. For example, in one particular plot development scene where $\mathrm{MC}^{\prime} \mathrm{s}$ best friend begins smoking at a family picnic which is attended by various family members, the auntie reproaches the best friend for smoking in Jade's presence (see figure 6.19). We hoped that in witnessing this exchange, players would be able to think more objectively about some negative consequences of smoking because $\mathrm{MC}$ is not the direct target of disapproval.

A further justification for this indirect approach is that collectivist cultures tend to be high context, meaning that people are closely observant of each other and read much underlying meaning into the words and actions of others $[59,62$, 


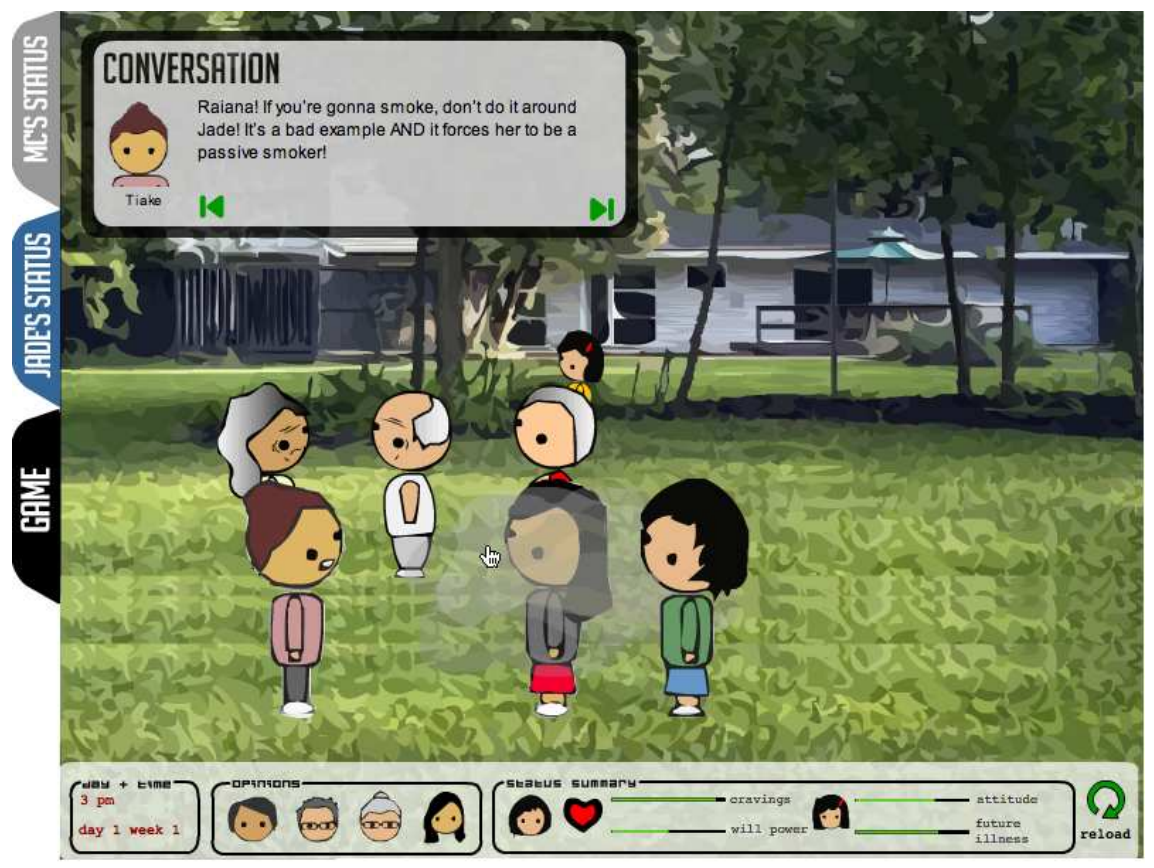

Figure 6.19: Auntie directing her thoughts on smoking at the best friend

$96,143]$. As we were designing for a collectivist player audience, it was a reasonable assumption that players might closely observe the experiences of the other characters, and apply their learnings to MC, and potentially even themselves. 


\section{Chapter 7}

\section{Smoke? Play testing and refinement}

During the development process of a game, a step considered critical in maximising its quality is the play testing phase [49]. Play tests are carried out on prerelease game prototypes, and conducted with potential players. A key rationale underlying play testing is that by trialing game prototypes, designers can refine aspects of the game that were not well-received by play testers prior to final release. These aspects can cover a range of issues, from identifying problems in game flow and challenge, to awkward game controls, and also to game usability issues and functionality glitches.

Play testing is commonly employed in the development process for commercial games, but has equal value for the development process of persuasive games. At the end of the game development process for Smoke?, our intention was to test the persuasiveness of its two versions, as opposed to its general design and whether it presented major usability issues. To identify significant game design issues early, we decided to conduct user and expert evaluations on initial prototypes of Smoke? We planned to use what we learned during these evaluations to shape how we approached the final iteration of development, and how we might need to modify the design directions we were taking with the game.

In this chapter, we conclude presentation of our second contribution, and focus on the design and usability issues that emerged from the play tests of the initial prototypes of the Smoke? game. We also discuss how the identified problems influenced development of the final prototypes of Smoke? 


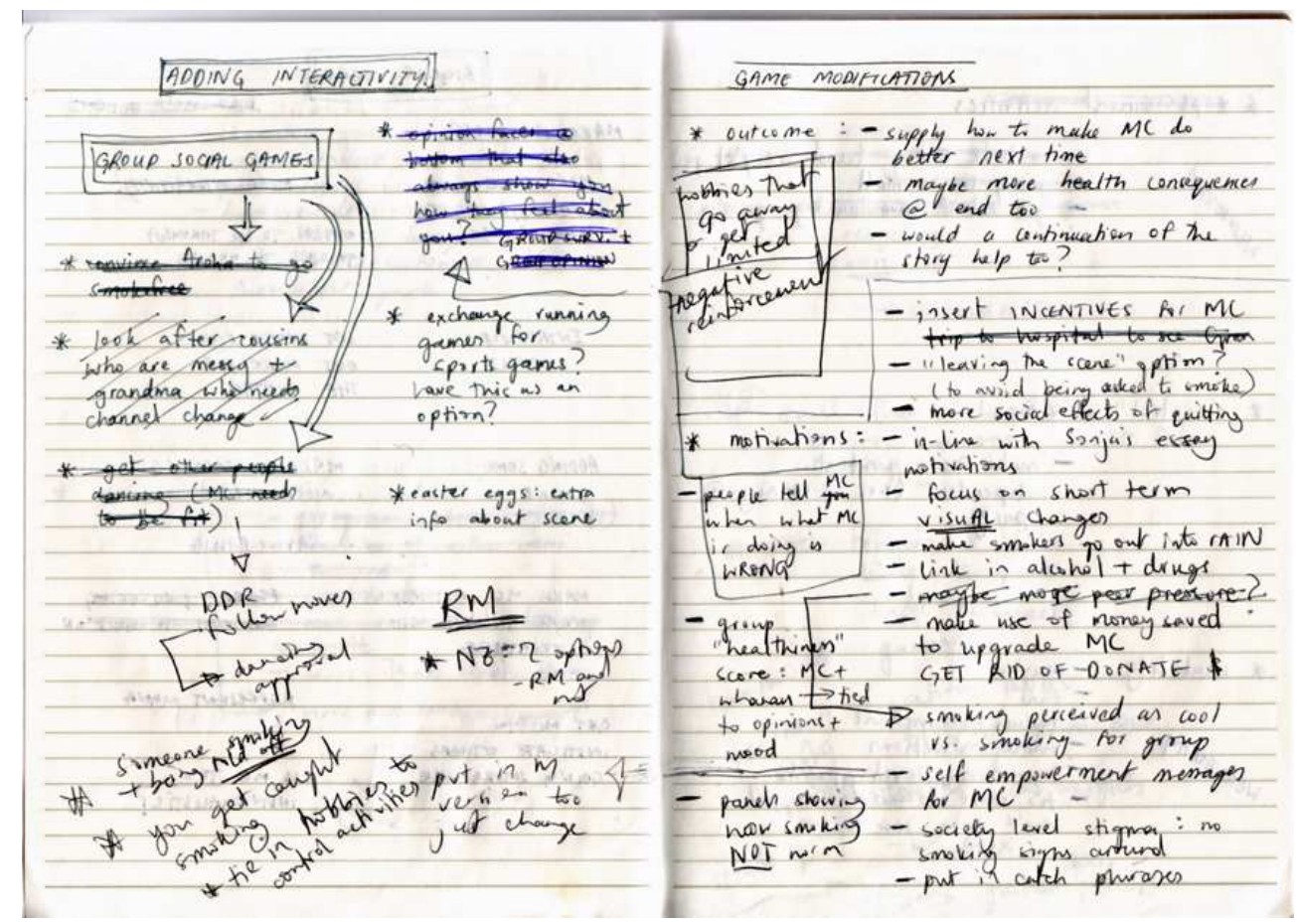

Figure 7.1: Notes from the design journal on change suggestions

\subsection{Play testing details}

\subsubsection{User evaluations}

\subsubsection{Motivation and process}

During product development, designers and developers often fall into the trap of assuming that users share the same cultural assumptions as them, and furthermore, will interpret and react to products in the same way as they do. To assess whether we were satisfying the criteria of our initial game design goals (see §5.1.2), we conducted a pilot user evaluation of both versions of Smoke? with potential players, including high school and university students. Additionally, we were interested in obtaining opinions on the game representations of Māori culture and smoking cessation, so we also involved Toihuarewa members and smoking cessation experts as evaluation participants. In §3.3.2.7, we discussed the details of the players involved in the pilot user evaluations in greater depth.

In conducting the pilot user evaluations, we used two methods: the thinkaloud protocol (see $\S 3.4 .2 .3$ ) and interviews (see $\S 3.2 .2 .1$ ). Once we had gathered significant feedback, we analysed the test findings, and grouped them into key themes. 


\subsubsection{Structure of the user evaluation themes}

In the upcoming $\S 7.2$, we present four themes drawn from the findings of the pilot user evaluation: managing player attention, balancing replay with reality, player control vs. system control, and identity issues. We discuss each theme by first describing the theme-related issues as encountered by players in the game. We then reformulate the central issues of each theme in terms of PT strategies, and use the strategy-informed discussion to work towards solutions. The PT strategies we refer to in $\$ 7.2$ include REDUCTION, TUNNELLING, TAILORING, SELF MONITORING, SUGGESTION, and CONDITIONING. We overviewed these six strategies in $\S 2.1$. We also refer to several PT strategies we developed and explained in $\S 4.3$ : MENTORING, GROUP CUSTOMISATION, and ACHIEVEMENT.

In real world products, PT strategies are rarely used in isolation [46]: their categorisation as distinct constructs is a useful analytical tool, but during the design process, the boundaries between strategies become significantly more fluid. The persuasive impact of any particular strategy as distinct from any other is difficult to pinpoint.

It is interesting to note that although our pilot user evaluations were performed on both the NZE and NZM versions of Smoke?, the prevalently referenced strategies in $\$ 7.2$ are ones we discussed as more suitable for individualist audiences, back in $\S 4.2$. In claiming in $\S 4.2$ that the motivations of certain strategies suited individualists more than collectivists, we did not mean that the strategies would be ineffective on collectivists, but that, in general, their effectiveness would be more pronounced in individualists rather than collectivists. For example, if the same individualist-oriented persuasive technology is used by individualist and collectivist audiences, we claim that it is more likely to be effective on individualist users, as compared to collectivist users.

Following on from this point, in deciding to make Smoke? a simulation/adventure game, we chose to adopt established games genres, which required adherence to a set of game design conventions. These conventions necessarily already contain groupings of embedded PT strategies. More broadly speaking, existing game genres can be characterised by particular groupings of PT strategies, many of which we have claimed are better suited to individualists (see $\S 4.2$ ).

\subsubsection{Expert evaluations}

\subsubsection{Motivation and process}

The purpose of the pilot user evaluations was to inform major decisions about the overall game design, some of which were clearly related to usability issues in the 
game's interfaces and were affecting how participants played. Traditional usability concerns are crucial in the PT design process. While a successful PT interface should suggest to users as to how it can facilitate them in changing their attitudes/behaviours, at the same time, the interface needs to remain unobtrusive. It is important not to shift users' focus from their persuasion goals, or distract them from other unrelated tasks they are also trying to perform. For these reasons, we decided to revisit the explicitly usability-related issues and conduct a cognitive walkthrough on the game. In $\S 3.3 .2 .8$, we overviewed the cognitive walkthrough method, and provided some details about our expert evaluation team.

The cognitive walkthrough method was devised as a tool for usability studies, and is usually employed in studies of productivity applications [153]. Typically, evaluators are told which tasks to complete. As productivity applications are often designed to support users performing particular tasks, the task-centric orientation of the walkthrough method makes it well-suited to evaluations of this nature.

It is not clear, however, how the task-centric focus of productivity application evaluation applies to play testing, which involves not just usability evaluation of a game, but also an evaluation of its controls, flow, pace, and challenge, among other aspects. An essential component of a well-designed game interface is that players are able to determine, usually from the interface itself, the tasks it makes sense for them to complete in terms of the overall game objective [17,52]. Additionally, depending on the nature of the game, establishing how to use the various elements of the game's interface may partly comprise the game play itself [18]. Explicitly instructing players how to use the game interface is counter-productive in play testing.

To repurpose the walkthrough method to evaluate usability aspects of our game, we gave the expert evaluation team two meta tasks. One such task was to complete a best behaviour play-through of the NZM version of Smoke? in which they were trying hard to keep MC smoke-free, while the other was to complete a worst behaviour play-through of the NZE version, in which they could make MC smoke as much as they liked. We gave no further detail of tasks to accomplish during the course of play. It was up to the evaluators to guess at each step what it seemed logical to do in terms of progressing the game state and to stay on track with the game play-through objectives.

As problems in the game interfaces were encountered, they were documented, along with an assessment of why they were likely to be problematic for players. We then grouped and prioritised the encountered problems and summarised them into significant themes. 


\subsubsection{Structure of the expert evaluation themes}

In the upcoming $\$ 7.3$, we present four themes summarising the key issues identified by the expert evaluators: use of "traditional" interface norms, feedback about changes to game state, reduction of text instructions, and fixing game world inconsistencies. We have not tried to relate this set of themes to PT strategies as we have done in $\S 7.2$, but aimed to focus our discussion on usability. For each expert theme, we open the discussion by providing some background on our design intentions during the development process. We then describe the issues the experts identified as problematic for particular features of the interface. Finally we discuss whether it was feasible to develop a solution for the problems given time constraints, and if so, we outline what we did.

\subsection{User evaluation themes}

\subsubsection{Managing player attention}

\subsubsection{Unintended consequences}

Scores and status representations are a common feature of many games. The first version of our game featured a status screen, as seen in figure 7.2, that charted eight continuously changing aspects of $\mathrm{MC}^{\prime}$ s health and well-being. During the initial design phase, we decided to include health information. We believed it served as a valuable opportunity to learn about the effects of smoking. The status screen was designed like a browser tab and could be viewed at any point during play. The status screen components were also summarised as an overall well-being score in a mini status summary window in the lower right-hand corner. Our evaluation participants, however, brought to our attention a couple of serious issues with the status screen. One participant claimed that there were too many aspects to keep track of and felt cognitively overloaded. Other participants observed that they hardly referred to the status screen. This was an important issue to address, because we believed that there was important information in the status screen.

\subsubsection{Reformulation in PT strategies}

The issue of cognitive overload can be recast as a tension between several, conflicting PT strategies: REDUCTION and TUNNELLING versus CONDITIONING, SELF MONITORING, MENTORING, and SUGGESTION (see $\S 2.1$ and $\S 4.3$ for an overview of these strategies). Linear narratives are an application of REDUCTION and TUN- 


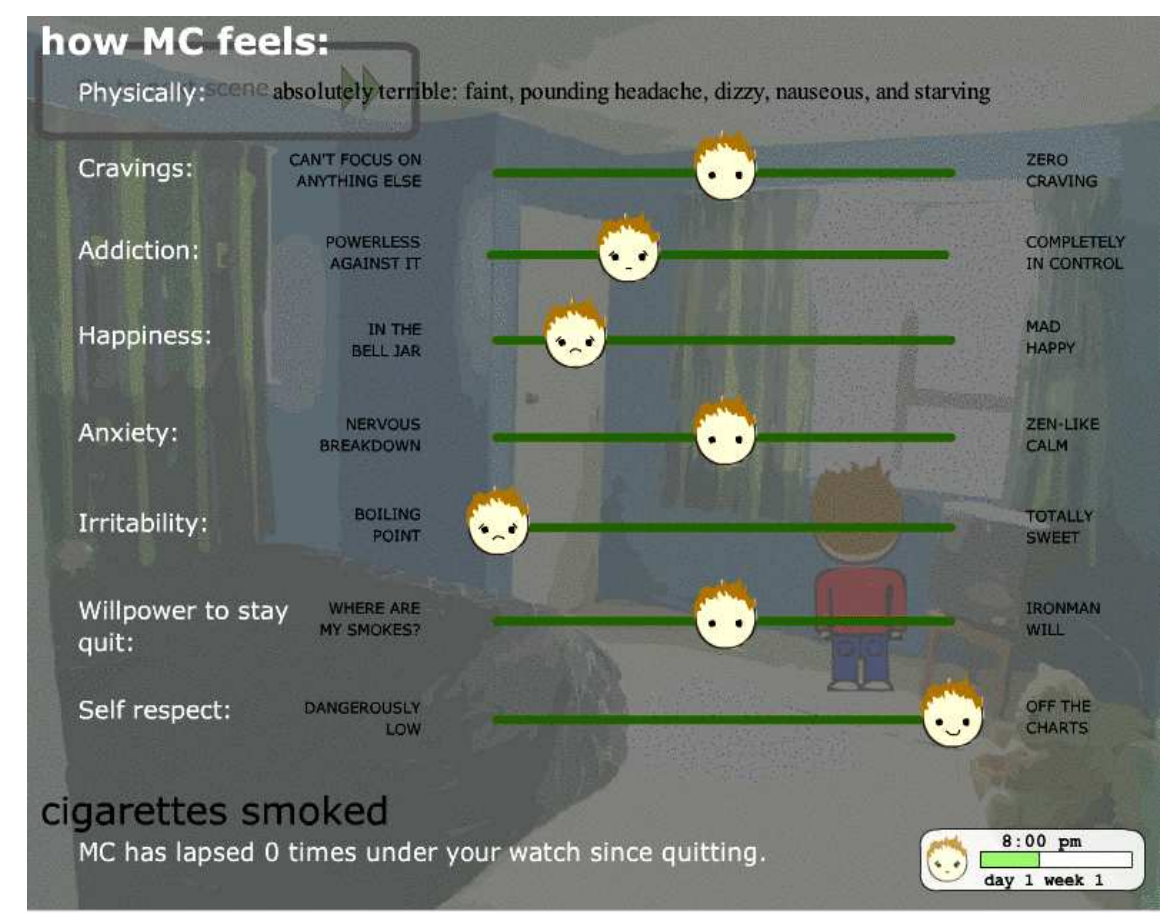

Figure 7.2: The initial version of the status screen

NELLING. Narrators control what their audience pays attention to and, to some extent, how they arrive at particular understandings. Games with clearly defined narratives thus employ REDUCTION and TUNNELLING, predetermining the path of players and focusing their attention on particular game elements. Score and status representations, on the other hand, can be considered as a shared function of CONDITIONING, SUGGESTION, SELF MONITORING, and MENTORING, in the following way. Changes in score, indicating progress, are presented as CONDITIONING feedback to players. Because players are responsible for their game progress, this information then facilitates SUGGESTION, SELF MONITORING, and MENTORING, by suggesting ways that they might improve performance.

The result that several of our participants were more interested in progressing along the narrative path of the game than in checking the status page is evidence that we had indeed succeeded in tunnelling their attention. Unfortunately, their attention had become too focused on the narrative. The game design was not effective in drawing their attention to status change feedback information. Players were missing out on two potential benefits. They were losing opportunities to learn how their game performance could be improved, and at a practical level, they were missing opportunities to relate their game status information to the real world consequences of smoking.

The strategies clearly needed to be rebalanced. Players needed to be interested enough in the game narrative to continue progressing, but also remain aware of feedback information so that they were willing to pay attention to suggestions 


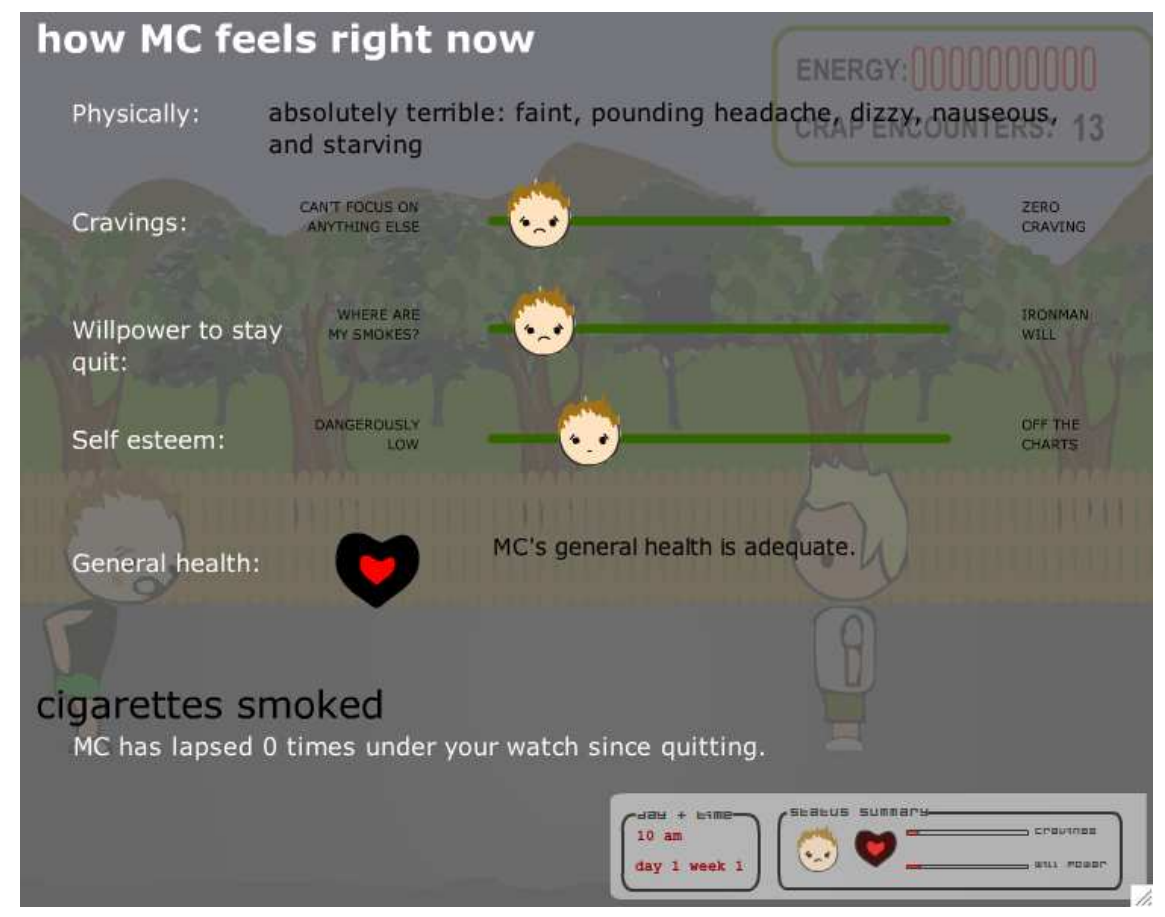

Figure 7.3: The final version of the status screen

about performance improvement. Further, once the status change feedback had been noted, players needed to smoothly transition back into the world of the game narrative in order to apply them. Another way to look at this issue is in terms of a cost-benefit analysis. One of our participants commented that she felt overloaded with status information: comprehension of the status information was causing her a cognitive processing overhead that was deemed too costly. The REDUCTION strategy seemed a straightforward way to reduce perceived cost, so we redesigned the status screen to report on only five elements, as in figure 7.3. This decision was made on the basis of whether we thought particular status information was integrated enough into the game narrative to contribute to its progression. In this way, we were also trying to facilitate smooth transitions back into the game narrative. To further ease the transition between the status and narrative screens, we modified the mini status summary window to provide more specific information. Another solution which we considered, but did not implement because of time restrictions, was adding incentives for players to check their status screens. Incentives are a typical way of balancing a cost-benefit ratio with extra benefits [33], and can be viewed as a form of CONDITIONING. With a simulation, however, designers must take care that they do not insert too many artificial motivators, otherwise they run the risk of the simulation having reduced relevance in real world contexts. 


\subsubsection{Balancing replay with reality}

\subsubsection{Unintended consequences}

Part of the power of simulation is its ability to represent real world cause-andeffect relationships [46]. With certain phenomena, the causality chain may be at least a few links long. Typically, digital games support some degree of replayability and players are likely to expect a replay option. We were concerned that adding replay would confuse the game narrative, in that cause-and-effect chains would be far less obvious, and the intended learning outcomes might become ambiguous. In the first iteration of game design, we decided not to allow players to replay portions of the game. One of our evaluation participants, however, noted that she would have liked to replay certain parts of the game, in order to redress unintentional choices she had made for MC that had resulted in outcomes she had hoped to avoid. She claimed that playing the game all the way through to achieve a sub-optimal outcome was disillusioning, and made her feel like "giving up". Failure to find this feature led to frustration and the detrimental consequence of the participant paying less attention to the game message.

\subsubsection{Reformulation in PT strategies}

The replay example relates to a tension between REDUCTION and TUNNELLING on one side, and CONDITIONING on the other. Cause-and-effect simulations are closely linked to the concept of narrative, and therefore relate to REDUCTION and TUNNELLING. The attention of users is directed along specific paths to prompt particular understandings of phenomena. Replay points, which can be likened to TUNNELLING exit points [46], can also be construed as tools players use to improve their chances of obtaining positive feedback, and in this way, relate to CONDITIONING. In the quest for maximising positive feedback then, players seek out ways to redo actions they have decided were not beneficial. They seek a TUNNELLING exit point, so that they can re-enter the tunnel, having optimised a prior game state, which may lead to receiving more positive feedback.

The first version of the game made use of TUNNELLING with no exit points. When tunnels of this nature are short, users are less likely to notice that they are being tunnelled, and therefore less likely to become frustrated at not being able to leave the tunnel. Smoke? however, featured long tunnels, to illustrate real relationships between smoking, health, and social situations. Failing to find a replay point, the participant, whose experience we recounted previously, felt that she had been denied hypothetical positive feedback. To remedy this situation, an obvious solution was to introduce TUNNELLING exit points. But we were concerned 


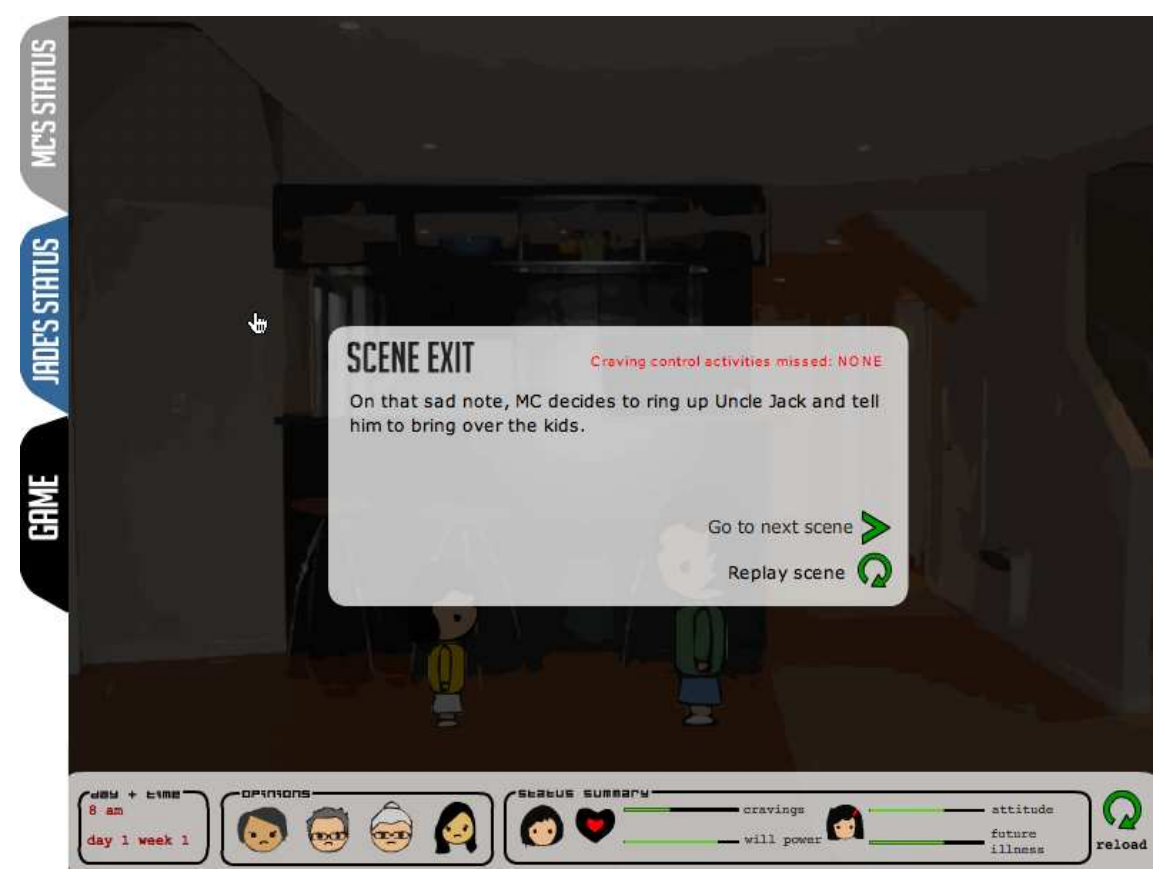

Figure 7.4: replay functionality representations in the game

that in introducing too many exit points, the benefits of TUNNELLING would be reduced, as it would become unclear as to where tunnels began, how players had reached certain game states, and consequently, what interpretations they would take away. Additionally, in real life, as in true simulation, there is no undo or redo option. In making it available we would detract from the original goal of modelling the real world.

Our solution was to introduce replay to some parts of the game. We allowed players to reload both during and at the conclusion of plot development scenes, as in figure 7.4. This addition of replay was a concession to players' desires for maximising potential positive feedback. In a way, the presence of replay even enhanced the game's capacity for modelling real world situations, as it increased players' immediate opportunities for exploring underlying cause-and-effect relationships. Bogost refers to this type of game persuasion as procedural rhetoric, that is, persuasion through the representation of processes [24]. At the same time, the inclusion of replay throughout a simulation game is a balance of two factors: providing enough exit points so that players can immediately address particular types of feedback information, and providing few enough exits so overall paths are discernible and a semblance to reality is maintained. 


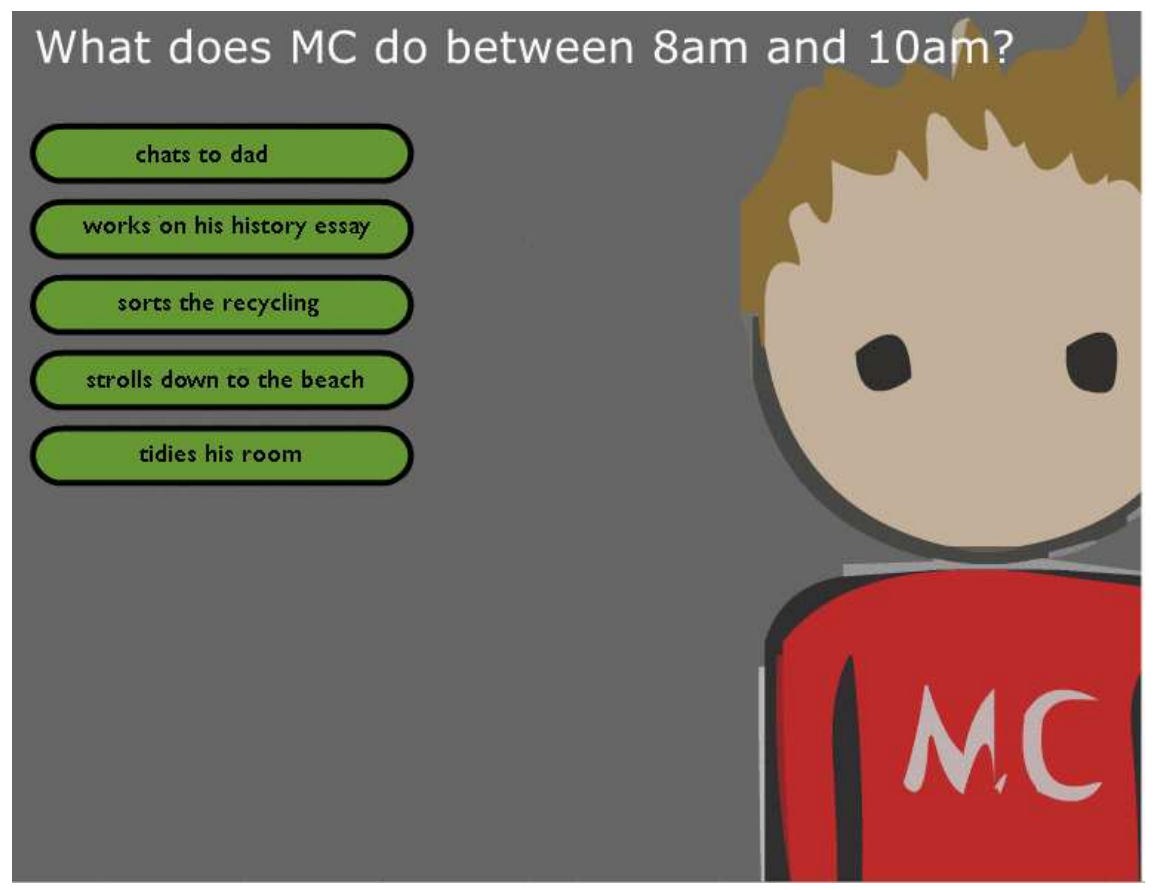

Figure 7.5: The initial version of the wildcard scene interface

\subsubsection{Player control vs. system control}

\subsubsection{Unintended consequences}

Digital games by necessity facilitate player activity. Given our developmental time and budget constraints, we designed our prototypes so that parts of the game play concentrated on simulation, while others, such as wildcard scenes, were dominated by system-led progression of game narrative. Several participants commented that they would have liked to have been able to complete activities in the game world that were not available. For example, to take MC shopping for clothes, and not just groceries, which was all the functionality the game supported at the time ${ }^{1}$. While some players wanted expansion of the game, others seemingly wanted more control. As discussed in $\$ 5.2 .2 .4$, to speed up the game progression, we introduced wildcard scenes throughout the game. In wildcard scenes, players are presented with a series of choices for how they think MC should spend the next two hours, and each choice has an unpredictable effect on MC's mood. We believed this had parallels with the experience of quitting in the real world, where many aspects of life are beyond personal control. Yet some of the participants were frustrated that they were not in control of every aspect of MC's state.

\footnotetext{
${ }^{1}$ Although we had already planned to include this functionality in the final version.
} 


\subsubsection{Reformulation in PT strategies}

The frustration players felt at not being able to complete or control particular activities in the game world may be related to the TAILORING, GROUP CUSTOMISATION, and ACHIEVEMENT strategies (see sections 2.1 and 4.3 for an overview of these strategies). Smoke? features certain affordances of customisation: for example, players can choose who MC talks to, how MC interacts with them, and what $\mathrm{MC}$ does to manage his cravings. In turn, this affects $\mathrm{MC}^{\prime} \mathrm{s}$ relationships with people, and how well he copes with life. We wanted players to control particular aspects of their game experience because we wanted them to generate their own outcome. When people believe that information is particularly relevant to them (and we argue that this is true whether it pertains just to them or to the group they are part of), they tend to find it more persuasive [46, 114]. In Smoke?, however, some of our participants interpreted real affordances of customisation as pointers to other affordances that they perceived but which were not in fact available to them. In turn, the mismatch between their expectations of what the game supported and the reality led to frustration. One obvious but not possible way to minimise the mismatch was to implement and support a greater range of player activity. At the same time, however, it seemed clear that there was a trade-off between TAILORING, GROUP CUSTOMISATION, and ACHIEVEMENT, and simulation, which can be represented using REDUCTION and TUNNELLING.

TAILORING, GROUP CUSTOMISATION, and ACHIEVEMENT are about supporting the goals of users and generating content or experiences of particular interest to them; they emphasise the importance of identity, and how identity affects outcomes. Simulation, on the other hand, represented through REDUCTION and TUNNELLING, typically describes known cause-and-effect relationships, or known relationships between particular entities; it is about emphasising predictability and how phenomena behave in pattern-regulated ways.

In the case of our game, the latter was the overriding motivation. It was more important for us to represent patterns to players, and retain control over what information the players would take away from the game than to fulfil players' expectations of game control and content. We did, however, redesign the game interfaces to contain clearer descriptions of what actions could be completed, and indicators for when $\mathrm{MC}$ is not under the complete control of players, as in figure 7.6. 


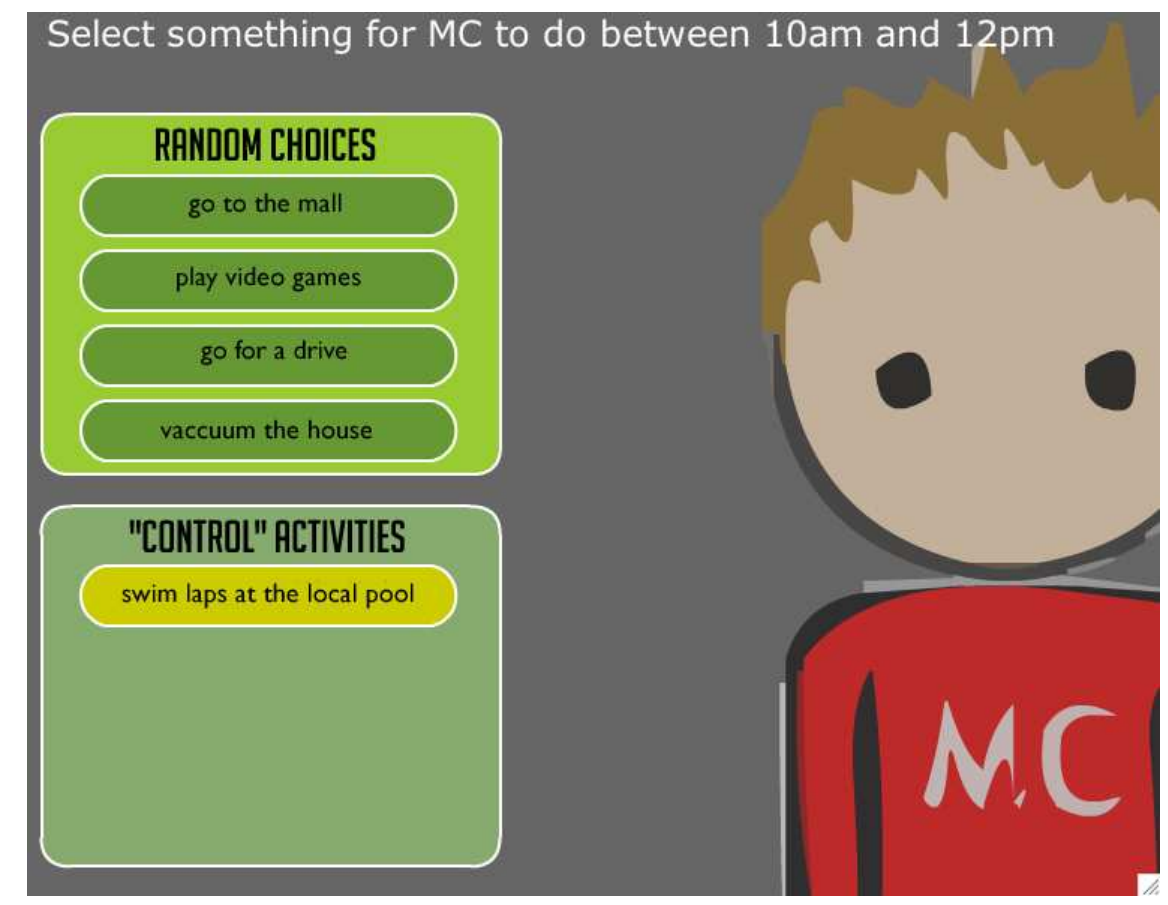

Figure 7.6: The final version of the wildcard scene interface

\subsubsection{Identity issues}

\subsubsection{Unintended consequences}

The introductory sequence of Smoke? informs players that they are to "guide MC to make choices" for six weeks of game time. Interviews with the participants revealed that different participants had quite varied ideas about their relationship with MC as an avatar. A couple of the younger participants discussed this relationship as one of friendship, so they described $\mathrm{MC}$ as a friend who they were helping. An older participant described feeling responsible for $\mathrm{MC}$, and was worried and guilty when MC started to suffer from craving-induced shaking. Another participant described how she played the game by identifying herself as MC. Yet another viewpoint expressed by a participant during a think aloud session was that she was attempting to "get into MC's head". At several points during play, this participant even asked aloud, "What would MC do?" Players' interpretations of their relationship with the avatar are likely to affect how they understand and internalise the game content. For example, if players feel responsible for $\mathrm{MC}$, and view MC as dependent on them, then it seems possible that $M C^{\prime}$ 's success or failure may motivate them to learn how to increase MC's chances of staying quit. In turn, they have a greater chance of internalising generalised knowledge about quitting behaviour. On the other hand, if players view MC as an entirely independent, pre-formed entity, then it is ambiguous as to whether they would be willing to explore how to reach a quit state for MC. These play- 
ers may miss out on valuable information about the consequences of different actions.

\subsubsection{Reformulation in PT strategies}

The issue of how players perceive the player-avatar relationship relates to TAILORING, GROUP CUSTOMISATION, SELF MONITORING, and MENTORING. TAILORING and GROUP CUSTOMISATION are partly about convincing users that that they are able to identify with content they encounter. The participant who asked "What would MC do?" clearly did not feel that MC was customised to be like her. In fact, her question leads us to believe that she felt it was easier to try and think like MC might, in the hypothetical world of Smoke?, than to make MC behave as she would herself in the real world. As discussed earlier, increased customisation leads to players paying more attention to outcomes. Maintaining player interest through an increased level of player-avatar identification is important for a couple of reasons. Firstly, it increases the likelihood that players are internalising information presented in the game, which is a key objective for any persuasive game. But secondly, the identification with the avatar can lead to a form of interest and motivation similar to that which drives SELF MONITORING and MENTORING. Once SELF MONITORING and MENTORING become established as motivational drivers, players are more likely to be open to experimenting with ways to push MC in different directions, and thus likely to learn more from the play experience.

To prompt a more dependency/responsibility view of the player-avatar relationship in players, we added more language cues to different parts of the game reminding players of how we would like them to view MC. As discussed in $§ 7.2 .3$, however, support for total customisation in Smoke? is not possible. Nonetheless, alerting players to some ways in which they can customise $\mathrm{MC}$, or do have control over MC, may lead to greater player-avatar identification, and some of its associated benefits.

\subsection{Expert evaluation themes}

\subsubsection{Adopting "traditional" interface norms}

\subsubsection{Game design context}

As we discussed in $§ 5.1$, one of our early development decisions was the choice of Flash as an application platform. An interesting consequence of this choice, however, soon became obvious during the walkthrough. The evaluators expected 


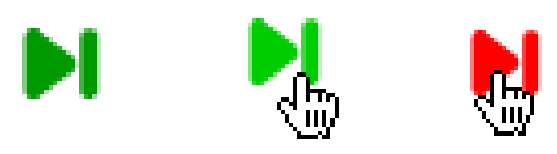

Figure 7.7: Off, over, and press states of the conversation window forward button

the game interface to function similarly to other standard computer applications. One example relates to button interactivity: in Smoke? all the buttons were defined so that their associated behaviour was activated on a press event, rather than a release event, as release is typical of many desktop computer interfaces. Another example relates to the button graphics used in the conversation windows: one evaluator pointed out that the forward graphic was identical to the fast-forward icon typically used in music and remote control interfaces, and that this mismatch was confusing.

\subsubsection{Examples and solutions}

While it was problematic that evaluators were making incorrect assumptions about the interface, the assumptions could be viewed as useful. The fact of their existence implied that some interface design conventions were common enough that we, too, could use them in our game interface, and expect them to be understood by the players, without the requirement of additional explanation.

Some of the "traditional" interface design notions we included in the game to harness common UI design conventions included:

- Modifying all button behaviours to be activated on the button release, as opposed to the button press.

- Modifying all buttons to provide players with visual feedback when they were moused over, or clicked (as in figure 7.7).

- Greying out interface components to signify that they had been temporarily disabled.

- Adopting a standard set of play icons for the buttons controlling the viewing of conversations.

- Removing interface elements that had either not yet been activated or explained to the player.

- Exclusive use of the hand icon to indicate clickable hot spots. 


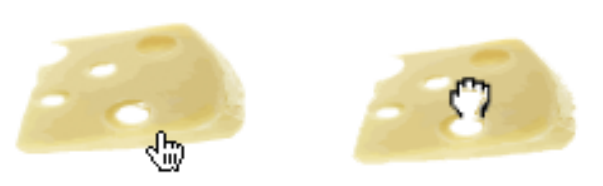

Figure 7.8: Pointing at a component vs. grabbing a component

\subsubsection{Feedback about changes to game state}

\subsubsection{Game design context}

A key reason for conducting cognitive walkthroughs is to determine whether users are likely to act and react to products as developers have assumed, even though the walkthrough is conducted by other developers [153]. A significant proportion of the usability issues identified in the walkthrough related to the evaluators feeling they had not been presented with enough micro-level feedback corresponding to changes in game states. This was relevant for changes arising directly as a result of their manipulation of the user interface, such as mousing over a hot spot, but also indirect game state updates linked to changes in the overall game context, such as updates in the moods + opinions box.

\subsubsection{Examples and solutions}

At the direct user manipulation level, one example of an identified problem related to one of the minigames, which involved dragging components around the screen. By default, when a button or movieclip is detected in a Flash application, the cursor changes to a pointing hand. We had defined the minigame components to become draggable on a mouse-press event, but the cursor remained as a pointing hand. One evaluator pointed out that the pointing hand dragging an item was a confusing mix of metaphors, and that players might not connect dragging with the mouse-press event. We addressed this problem by adding a different grabbing cursor image to represent dragging/grabbing once a mousepress event had been detected on a draggable item. Figure 7.8 shows an example of different cursors used during object grabbing.

At the indirect game state change level, a glaring problem was identified with the moods + opinions box at the bottom of the game screen. Any character icon in the moods + opinions box could have one or both of two kinds of state changes: a mood state change or an opinion change. Unfortunately, the evaluators hardly noticed either of these changes. Compounding this problem was the issue of screen real estate. We believed that the moods + opinions box could serve as an important source of information for players, yet we wanted to keep most of the central 


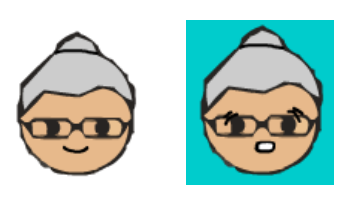

Figure 7.9: A mood + opinion face before and after a mood change

portion of the screen free for representing the game scene and characters. Accordingly we had decided to position the moods + opinions box at the bottom of the screen, but it was apparent that the evaluators were oblivious to state changes in that area. To correct this problem, we modified the moods + opinions state change notifications to more aggressively demand attention: the mood state change was changed to depict a bright blue background behind the face (as in figure 7.9), while the appearance of a new opinion was denoted by the face flashing until it was clicked on by players.

\subsubsection{Reduction of text instructions}

\subsubsection{Game design context}

Smoke? is a narrative driven game, in which most of the game is progressed through MC's conversations with other characters. In playing the game, players spend a lot of time reading conversations. One evaluator pointed out that as well as needing to read narrative progressions, he was also required to read instructions relating to how to play the minigames, and the specialised modes of interaction needed to play them. Our initial reasoning for including the minigame instructions moments before commencing minigame play related to our general solution to the problem of how to deal with game instructions. As we had decided not to create instruction manuals, we commenced the game with a tutorial explaining the basic interactions possible, as well as overall objectives. We were concerned that if we attempted to supply players with all of the instructions at the beginning, they would become too overloaded with information. Alternatively, we thought players would become bored and ignore the rest of the tutorial. For these reasons we decided to explain the minigame instructions inline with the minigames themselves. We anticipated that the players would have a higher chance of remembering the instructions because they could immediately experiment with them. The evaluators illustrated, however, that the abrupt change of interaction style between the main game and the minigames was problematic. In addition, although instructions were presented explaining the new interaction styles, the evaluators were tired by reading conversation text, and did not pay close attention to the instructions. Consequently they struggled to work out how 

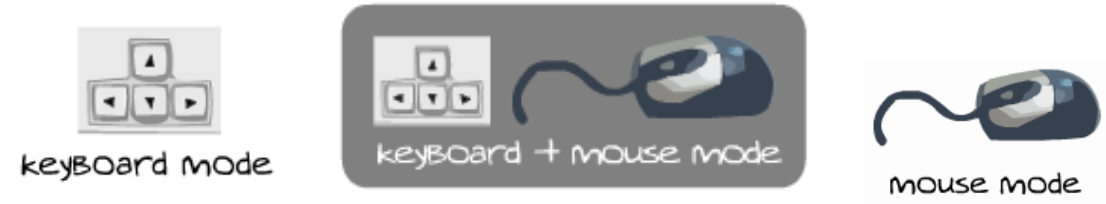

Figure 7.10: Three interaction mode icons

to play the minigames.

\subsubsection{Examples and solutions}

With the time limitations in our development schedule, we only provided a partial solution to the problem of text instruction overload. Where feasible, we reduced the amount and added interaction mode icons, such as those in figure 7.10. They represented the current modes required, appearing and then unobtrusively fading out. Other solutions we would have put in place with more time included a pause function, during which instructions for the game could be viewed.

\subsubsection{Fixing game world inconsistencies}

\subsubsection{Game design context}

We modified the walkthrough process to analyse the game's interfaces, because we thought it was important that the evaluators establish how to reach a particular game state on their own. Consequently, there were no step-by-step task directives given. A typical component of game play is experimentation with the game interface, by exploring the interactive possibilities available, and even going as far as seeking to "break" the interface [18]. Interestingly, interface exploration is less common in the case of productivity applications (in comparison to games), where users are unlikely to take the time to try and discover the boundaries of interaction.

It was clear that the evaluator in charge of driving the interaction during the walkthrough was familiar and comfortable with games: he was a compulsive "clicker", seeking out all available screen hot spots, and testing game movement boundaries. As well as establishing the ways in which we had designed the game to respond to player interaction, he found several unexpected game responses that we had not thought to investigate. For example, he illustrated that it was possible to slowly click MC off the game screen boundary so that he was not visible. Other examples of previously undiscovered and unexpected behaviours included the ability to make one character walk over another with multiple clicks, and disrupting the verification algorithm in one of the minigames by clicking 
rapidly. While none of these problems were major enough to halt the evaluators from proceeding, they were inconsistent with the rest of the intended game interaction, and detracted in small ways from the game play and narrative.

\subsubsection{Examples and solutions}

In redeveloping the game, we addressed the inconsistency issues as much as possible, by tightening boundaries for interpreting "clicks". All the same, the experience of the walkthrough highlighted to us that it would be near impossible to address all of the minor usability problems present in the game's interfaces. 


\section{Chapter 8}

\section{Evaluation}

The purpose of conducting an evaluation of our culturally-relevant PT Smoke? was to investigate if players would express different attitudes towards smoking after playing either version. At a more specific level, we were interested in finding out whether individualist players would find the NZE version of Smoke? more persuasive than the NZM version, and equally, whether collectivist players would be more persuaded by the NZM version than the NZE version. As we discussed in $\S 2.1 .3$, there is no clear consensus on how to evaluate the effectiveness of persuasive games on attitude change. As such, we decided to adopt both a quantitative and qualitative approach in assessing the effectiveness of Smoke? In this chapter, we present our final research contribution: an evaluation of our culturally-relevant PT game Smoke? We overview the findings of two forms of evaluation we conducted on both of its versions: a large scale quantitative evaluation, involving 141 participants of both individualist, and collectivist cultures, and a small scale qualitative evaluation, performed with 8 participants, 4 of whom self-identified as NZ European, and remaining 4 of whom self-identified as Māori.

\subsection{The quantitative evaluation}

\subsubsection{Method}

In the cross-cultural psychology literature, pre- and post-surveys are the predominant method of tracking attitude changes following exposure to certain phenomena. We adopted this approach ourselves, by presenting players with surveys before and after playing a randomly allocated version of Smoke? once. Focusing on cultural orientation and game version, we decided to use a 2 (individualistic cultural orientation vs. collectivistic cultural orientation) $\times 2$ (NZE version vs. 
NZM version) factorial design.

\subsubsection{Subjects}

We recruited 141 participants to play Smoke? 100 were students enrolled in an introductory 100-level psychology course at Victoria University. The other 41 had either responded to flyers at the university campus, or were final year high school students from the greater-Wellington area who intended to continue with tertiary education. The psychology course students were awarded course credit for taking part in our study, while the other participants were rewarded with movie vouchers.

Of these 141 participants, 71 self-identified their ethnicity as NZ European, and 46 self-identified with the Māori or Pacific peoples ethnic group. The remainder of the participants identified with a range of other ethnicities, including Chinese and Indian ethnic groups. In addition, 43 described themselves as current smokers, 32 described themselves as ex-smokers, and the remaining described themselves as non-smokers.

\subsubsection{Study design}

The experiment session lasted approximately an hour for each participant. At the beginning of a session, participants completed a pre-survey, containing items about their value orientations, and attitudes to smoking. Next, they were randomly allocated one of the two versions of Smoke? to play: either the NZE version (designed to appeal to individualist players) or the NZM version (designed to appeal to collectivist players). After playing the game through once, the participants completed a post-survey, containing items related to attitudes towards smoking. At the end of the session, participants were debriefed about the experiment and the research.

\subsubsection{Measures}

Culture and values Our pre-survey contained a question on self-identified ethnicity. We categorised responses to the ethnicity question as follows: those who identified as NZ European were assigned "1", those who identified as Māori or as Pacific were assigned "2", others of European descent (including Australians and North Americans) were assigned " 3 ", and all others were assigned " 4 ". We were aware that self-identified ethnic identities might not necessarily be insightful, as multiple factors affect how people answer the questions, and the term ethnicity can also be ambiguous. Statistics New Zealand, for example, analyses 
census information to produce three Māori populations: the Māori descent or ancestry group; the Māori ethnic group, comprised of those who indicated Māori as at least one of their ethnic affiliations; and the sole-Māori group, those who indicated Māori as their only ethnic affiliation [135]. To address some of these concerns, one section of the pre-survey was dedicated to asking subjects about their value preferences, so that we could classify their value orientations as being more individualistic, or collectivistic.

The section on values contained 30 value items taken from Schwartz's values survey, which typically contains 54 items [128]. As discussed in $\S 2.2 .3$, Schwartz's values continuum contains an inherent bipolar dimension related to individualism and collectivism, or in Schwartz's terminology, an openness to change - conservation dimension. We selected the 30 values so that approximately half of them were more relevant to individualism (e.g. "INDEPENDENT (self-reliant, self-sufficient)"), and half were more relevant for collectivism (e.g. "HONORING OF PARENTS AND ELDERS (showing respect)"). We note that in theory, while the two dimensions are dependent and represent two sides of a continuum, in an analysis, they were shown to relate to Triandis's individualism and collectivism dimensions [108]. Triandis views individualism and collectivism as related, but distinct [144]. Consequently, the way people respond to openness to change items does not necessarily indicate how they respond to conservation items.

All of the value items could be ranked by the subject on a Likert scale of 0 to 7 , with 0 indicating that the value was completely unimportant to the subject, and 7 indicating that it was of supreme importance.

On collating the subjects' responses, we used the multi-dimensional scaling method to examine patterns in responses to the value items. A two-dimensional plot of subjects' responses showed that there were clusters around openness to change items, and another around conservation items. This was consistent with previous literature on value clusters in Schwartz's value theory $[47,128,130]$. Accordingly, we created two variables relating to the Schwartz value items. One corresponded to the subjects' degree of individualism (i.e. openness to change) and the other to subjects' degree of collectivism (i.e. conservation). We then compared means for the individualism and collectivism variables with people's own ethnicity categorisations. Those who were categorised as "NZ Europeans" and "other Europeans" had higher total means for individualism and lower total means for collectivism than those categorised as "Māori or Pacific", and "all other", who had higher total means for collectivism and lower total means for individualism. For the rest of our analyses, we used subjects' individualism and collectivism scores as measures of their cultural orientation: those with high individualism or low collectivism scores were considered individualist, while those with low individualism 
or high collectivism were considered collectivist.

Importantly, we treated both individualism and collectivism as continuous variables.

The items we refer to here are contained in question 1 of appendix I.

Perception of smoking Both the pre- and post-surveys contained 21 items about subjects' general beliefs regarding smoking, which were drawn from surveys used in previous studies on smoking cessation [30]. The items covered both shortterm consequences of smoking (e.g. "Smoking causes bad breath") and long-term ones (e.g. "Smokers have a higher risk of getting a serious illness"). They included nine positive effects beliefs (e.g. "Smokers have more friends"), alongside eleven negative effects beliefs (e.g. "Smokers are bad role-models").

These items could be ranked on a Likert scale of 1 to 5 , with 1 representing "Strongly Disagree", and 5 representing "Strongly Agree".

After the surveys were completed, we separated the beliefs items into a positive beliefs group, and a negative beliefs group. We determined a mean value for each subject's responses for the two groups. In upcoming discussion, we refer to the positive beliefs group mean as positive beliefs, and the negative beliefs group mean as negative beliefs.

The items we refer to here are contained in questions 2 and 9 of appendix I.

Smoking history Critical to our evaluation of the game was information about the role that smoking played in subjects' lives. We included survey items in the pre-survey about the number of close male and female friends subjects had who were smokers (e.g. "How many of your best four female friends smoke?"), as well as the number of family members and house-mates who smoked.

In the pre-survey, we also featured items in which smoker subjects were asked to classify their smoking behaviour (e.g. "Ex-smoker" to "Heavy smoker"), quantify their smoking frequency (e.g. "Once a month" to "Every day or nearly every day"), and describe how long they had been smoking (e.g. "Tried it only once" to "A few years"). These items of classification were obtained from a previous study [30].

From the survey responses regarding the role that smoking played in subjects' lives, we determined a mean value, which we refer to in upcoming discussions as proximity. From the smokers' responses regarding smoking behaviour, we determined another mean value, which we refer to as status.

The items we refer to here are contained in questions 6 and 7 of appendix I. 
Quitting intentions For subjects who were smokers, our pre- and post-surveys contained five items related to their intention to quit in the future, including "In the next week", "In the next month", "In the next three months", "In the next six months", and "Sometime in the next year".

Each item could be ranked on a Likert scale of 1 to 6 , with 1 representing "Very Low" and 6 representing "Very High". Similar items were used in a study by Dijkstra et al. [39].

From the surveys, for each respondent, we obtained two mean values related to their quitting intentions: one determined from their pre-survey quitting intentions, and the other from their post-survey quitting intentions. We refer to these means in upcoming discussion as pre- and post-intention to quit.

The items we refer to here are contained in questions 7 and 12 of appendix I.

Smoking resistance For subjects who were non-smokers, our pre- and postsurvey contained five items relating to resistance to smoking (e.g. "I am actively avoiding situations where people smoke").

The resistance items could be ranked on a Likert scale of 1 to 5, with 1 representing "Strongly Disagree", and 5 representing "Strongly Agree".

From subjects' responses, we obtained two mean values related to their resistance to smoking: one for their pre-survey responses, and one for their postsurvey responses. We refer to these means in upcoming discussion as pre- and post- resistance to smoking.

The items we refer to here are contained in questions 8 and 13 of appendix I.

Smoking temptation For subjects who were non-smokers, our pre- and postsurvey contained nine items relating to their temptation to smoke (e.g. "I am tempted to try a cigarette").

The temptation items could be ranked on a Likert scale of 1 to 5 , with 1 representing "Strongly Disagree", and 5 representing "Strongly Agree".

For each respondent, we determined two mean values related to their temptation to smoke: one for their pre-survey responses, and one for their post-survey responses. We refer to these means in upcoming discussion as pre- and posttemptation to smoke.

The items we refer to here are contained in questions 8 and 13 of appendix I. 


\begin{tabular}{|l|r|r|r|}
\hline Mean & $N$ & Pre-survey & Post-survey \\
\hline \hline positive beliefs & 140 & 3.9959 & 3.9367 \\
\hline negative beliefs & 140 & 4.0205 & 4.0873 \\
\hline resistance to smoking & 101 & 3.2129 & 3.3622 \\
\hline intention to quit & 40 & 3.9200 & 4.1125 \\
\hline temptation to smoke & 103 & 1.6096 & 1.5411 \\
\hline
\end{tabular}

Table 8.1: Pre- and post-survey means of attitudes, potential range: 1 to 5

\begin{tabular}{l|r|r|r|}
\hline Pair & Mean & Std. error mean & t ratio \\
\hline \hline positive beliefs & $-.05923^{*}$ & .03480 & -1.702 \\
\hline negative beliefs & $.06681^{*}$ & .02888 & 2.313 \\
\hline resistance to smoking & $.14934^{*}$ & .05585 & 2.674 \\
\hline intention to quit & $.19250^{* *}$ & .12102 & 1.591 \\
\hline temptation to smoke & $-.06848^{*}$ & .03222 & -2.125 \\
\hline \\
* Significant at the 0.05 level. \\
** Significant at the 0.10 level.
\end{tabular}

Table 8.2: t-test comparison of post- and pre-survey attitude means

\subsubsection{Analyses and results}

\subsubsection{Overall effects of Smoke?}

Alongside differences in players' attitudes in terms of cultural orientation and game version, we were interested in whether the game had had any overall effect on subjects' attitudes. Table 8.1 presents the details of the pre- and post-survey means for each of the groups.

To determine whether changes in the post- and pre-survey means were due to factors other than random variance, we performed a paired samples $t$-test, the results of which we report in Table 8.2.

The t-test revealed that the changes in post- and pre- means for positive beliefs, negative beliefs, resistance to smoking, and temptation to smoke were significant at a 0.05 level by a one-sided test. The change in post- and pre-intention to quit was significant at the 0.10 level by a one-sided test. Figure 8.1 displays a bar graph of the pre- and post-survey attitude group means.

The game was therefore generally successful in shifting people's anti-smoking attitudes. After playing the game, subjects reported an increase in negative beliefs towards smoking, an increase in resistance to smoking, and an increase in intention to quit. Subjects also reported a decrease in positive beliefs towards smoking and a decrease in temptation to smoke. 
A comparison of overall pre- and post-survey means

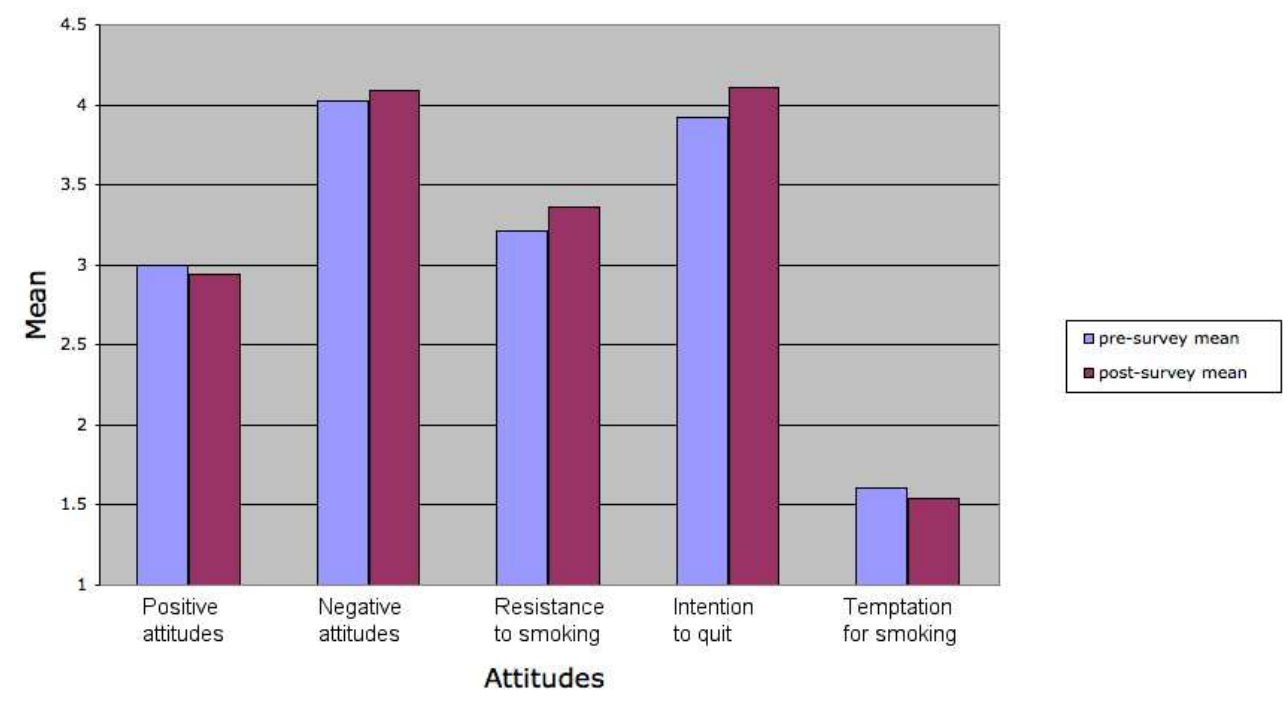

Figure 8.1: A bar graph of the paired means of five attitude groups

\subsubsection{Regression model of attitudes towards smoking by cultural orienta- tion and game version}

In $§ 8.1 .1 .3$, we discussed how we measured subjects' cultural orientations as continuous variables corresponding to subjects' degree of individualism, and degree of collectivism. Because we were dealing with continuous variables, we used regression to investigate dependencies between the subjects' post-survey attitudes, in light of their pre-survey attitudes, cultural orientations, and game version.

We used a multiple regression model estimated by the ordinary least squares method featuring the following variables as regressors: status: the mean variable relating to subjects' smoking behaviour, proximity: the mean variable relating to the importance of smoking in subjects' lives, version: a categorical variable describing the game version that subjects were playing: 1 for NZE, the individualist version of the game, or 0 for NZM, the collectivist version of the game), one of either individualism or collectivism: mean variables representing subjects' degree of individualism or collectivism, and finally interaction: a crossproduct interaction term representing the joint effect of version and one of either individualism or collectivism.

We then regressed each of the five post-survey means of positive beliefs, negative beliefs, resistance to smoking, intention to quit, and temptation to smoke on our model, including the corresponding pre-survey mean as an additional regressor.

The basic form of our regression equation was one of the following equations, both of which are identical apart from the variable measuring culture: 


$$
\begin{aligned}
\text { postmean }_{i}= & \beta_{1}+\beta_{2} \text { status }_{i}+\beta_{3} \text { proximity }_{i}+\beta_{4} \text { premean }_{i}+ \\
& \beta_{5} \text { version }_{i}+\beta_{6} \text { individualism }_{i}+\beta_{7} \text { interaction }_{i}+\text { error }_{i}
\end{aligned}
$$

\subsubsection{Significant interactions between cultural orientation and game version}

Intention to quit smoking By regressing post-survey means for smokers' intention to quit against regressors of status, proximity, smokers' pre-survey intention to quit, version, individualism, and interaction, we investigated the following hypotheses:

H1a: Players high in individualism values will have a greater shift in intention to quit smoking after playing the NZE version of Smoke? than after playing the NZM version.

H1b: Players low in individualism values will have a greater shift in intention to quit smoking after playing the NZM version of Smoke? than after playing the NZE version.

We summarise the details of the intention to quit regression model in table 8.3.

\begin{tabular}{|l|r|r|r|}
\hline Regressor & $\hat{\beta}$ & Std. Error & tratio \\
\hline \hline Constant & $1.988^{*}$ & .795 & 2.501 \\
\hline status & -.046 & .038 & -1.213 \\
\hline proximity & $.229^{*}$ & .060 & 3.794 \\
\hline pre- intention to quit & $.888^{*}$ & .056 & 15.946 \\
\hline version & $-2.039^{*}$ & 1.051 & -1.939 \\
\hline individualism & $-.418^{*}$ & .145 & -2.893 \\
\hline interaction & $.479^{*}$ & .199 & 2.140 \\
\hline \multicolumn{3}{|c|}{$n=43, R^{2}=.902$} \\
\hline
\end{tabular}

* Significant at the 0.05 level.

** Significant at the 0.10 level.

Table 8.3: Regression details for dependent variable: post-survey intention to quit

In the intention to quit regression model, the effect of interaction, the regressor term representing the joint effect of version and individualism, is significant at the 0.05 level by a one-sided test. In figure 8.2, we have plotted the change in smokers' post- and pre- intention to quit, by cultural orientation and game version.

Smoker subjects high in individualism values (i.e. individualist subjects) who played the NZE version of Smoke? demonstrated a greater shift in pre- and postsurvey intention to quit than those who played the NZM version. This result supports H1a. 
Intention to quit pre- and post-survey mean differences by version

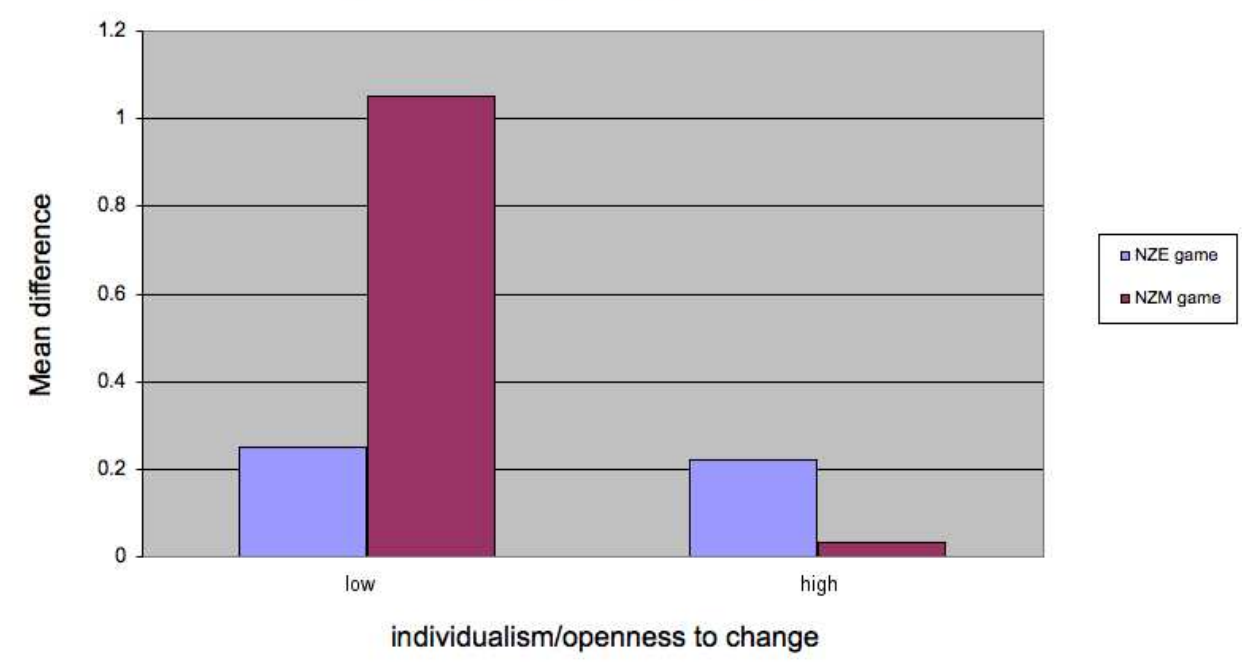

Figure 8.2: Changes in subjects' post- and pre- intention to quit by cultural orientation and game version

Smoker subjects low in individualism values (i.e. collectivist subjects) who played the NZM version of Smoke? demonstrated a greater shift in pre- and postsurvey intention to quit than those who played the NZE version. This result supports H1b.

Both these findings support our research hypothesis that cultural relevance is positively correlated with PT effectiveness. Interestingly, both game versions were both more successful in shifting attitudes of collectivist players than of individualist players. This may indicate that our individualist smoker subjects were less receptive overall to persuasion about quitting smoking than our collectivist smoker subjects.

Resistance to smoking By regressing post-survey means for subjects' resistance to smoking against regressors of status, proximity, pre-survey resistance to smoking, version, individualism, and interaction, we investigated the following hypotheses:

H2a: Players high in individualism values will have a greater change in resistance to smoking after playing the NZE version of Smoke? than after playing the NZM version.

H2b: Players low in individualism values will have a greater change in resistance to smoking after playing the NZM version of Smoke? than after playing the NZE version. 
We summarise the details of the resistance to smoking regression model in table 8.4.

\begin{tabular}{|l|r|r|r|}
\hline Regressor & $\hat{\beta}$ & Std. Error & $t$ \\
\hline \hline Constant & $1.241^{*}$ & .553 & 2.242 \\
\hline status & .012 & .024 & .494 \\
\hline proximity & $-.049^{* *}$ & .035 & -1.414 \\
\hline pre- resistance to smoking & $.786^{*}$ & .069 & 11.368 \\
\hline version & $-1.199^{*}$ & .660 & -1.817 \\
\hline individualism & -.058 & .092 & -.632 \\
\hline interaction & $.220^{*}$ & .125 & 1.758 \\
\hline \multicolumn{3}{|c|}{$n=107, R^{2}=.626$} \\
\hline
\end{tabular}

* Significant at the 0.05 level.

** Significant at the 0.10 level.

Table 8.4: Regression details for dependent variable: post-survey resistance to smoking

In the resistance to smoking regression model, the effect of interaction is significant at the 0.05 level by a one-sided test. In figure 8.3, we have plotted the change in subjects' post- and pre- resistance to smoking, by cultural orientation and game version.

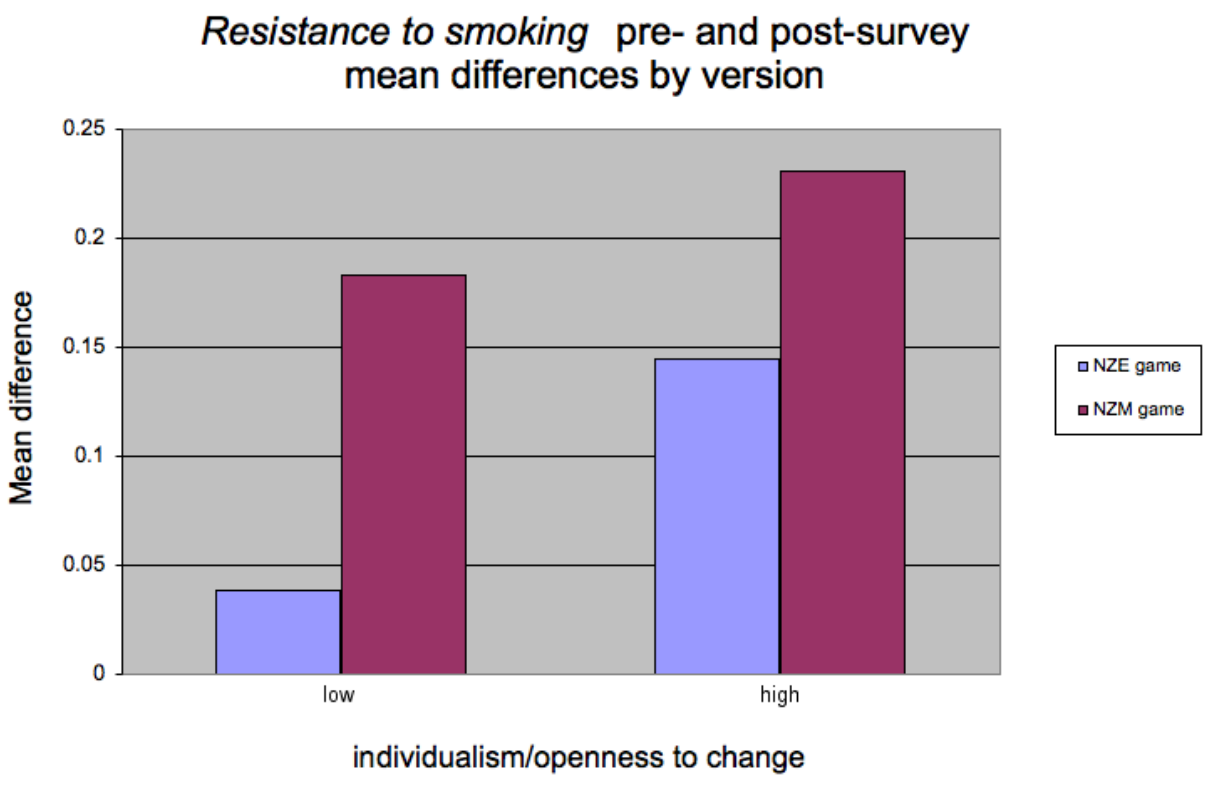

Figure 8.3: Changes in subjects' post- and pre- resistance to smoking by cultural orientation and game version

Subjects high in individualism values (i.e. individualist subjects) who played the NZE version of Smoke? demonstrated a lesser shift in pre- and post-survey resistance to smoking than those who played the NZM version. This result does not support $\mathrm{H} 2 \mathrm{a}$. 
Subjects low in individualism values (i.e. collectivist subjects) who played the NZM version of Smoke? demonstrated a greater shift in pre- and post-survey resistance to smoking than those who played the NZE version. This result supports $\mathrm{H} 2 \mathrm{~b}$.

At the same time, we note that individualist players of the NZE version of Smoke? demonstrated more than twice the attitude shift of collectivist players of the NZE version. So, while the NZM version appears to be more effective in increasing all players' resistance to smoking, the NZE version is comparatively more effective on individualist rather than collectivist players. We hypothesise that, despite our intention to design equally persuasive game versions, irrespective of cultural factors, aspects of the NZM version may simply have been more persuasive than the NZE version. Effective persuasive game design is a rather new area of research, and needs to mature before general design principles regarding persuasive games are established.

Interestingly, while resistance through collective support, as in the NZM version of Smoke? may seem more like a collectivistic concept, it is in fact a central feature of organisations like Weight Watchers and Alcoholics Anonymous. Both have been very successful in Western (individualistic) countries. This may indicate that for increasing attitudes related to different types of resistance, individualists may benefit from collective support as much as collectivists, if not more. We hypothesise that because it is less part of their everyday interaction, it has additional persuasion for them.

Temptation to smoke By regressing post-survey means for temptation to smoke against regressors of status, proximity, pre-survey temptation to smoke, version, collectivism, and interaction, we investigated the following hypotheses:

H3a: Players high in collectivism values will have a greater change in temptation to smoke after playing the NZM version of Smoke? than after playing the NZE version.

H3b: Players low in collectivism values will have a greater change in temptation to smoke after playing the NZE version of Smoke? than after playing the NZM version.

We summarise the details of the temptation to smoke regression model in table 8.5 .

In the temptation to smoke regression model, the effect of interaction, the regressor term representing the joint effect of version and collectivism, is significant at the 


\begin{tabular}{|l|l|l|l|}
\hline Regressor & $\hat{\beta}$ & Std. Error & t ratio \\
\hline \hline Constant & $-.350^{* *}$ & .225 & -1.554 \\
\hline status & -.003 & .028 & -.123 \\
\hline proximity & .070 & .171 & .412 \\
\hline pre- temptation to smoke & $.756^{*}$ & .072 & 10.514 \\
\hline version & $.673^{*}$ & .269 & 2.506 \\
\hline collectivism & $.158^{*}$ & .045 & 3.521 \\
\hline interaction & $-.171^{*}$ & .062 & -2.739 \\
\hline \multicolumn{4}{|c|}{$n=66, R^{2}=.705$} \\
\hline
\end{tabular}

* Significant at the 0.05 level.

** Significant at the 0.10 level.

Table 8.5: Regression details for dependent variable: post-survey temptation to smoke

0.05 level by a one-sided test. In figure 8.4, we have plotted the change in subjects' pre- and post- temptation to smoke, by cultural orientation and game version.

Subjects high in collectivism values (i.e. collectivist subjects) who played the NZM version of Smoke? demonstrated a lesser shift in pre- and post-survey resistance to smoking than those who played the NZE version. This result does not support H3a.

Subjects low in collectivism values (i.e. individualist subjects) who played the NZE version of Smoke? demonstrated a lesser shift in pre- and post-survey temptation to smoke than those who played the NZM version. This result does not support $\mathrm{H} 3 \mathrm{~b}$.

So interestingly, both of our hypotheses regarding temptation to smoke were contradicted by the findings of the regression model. These findings deserve some consideration. One way to interpret them in the context of our overall hypothesis is as follows. The smoking cessation literature has reported in depth on how cue exposure for smokers increases urges to smoke [27, 103]. We suggest that in the condition where subjects played a game version that reflected their cultural orientation, or a culturally-congruent condition, players were better able to engage with the game content by the context of their personal lives. Encountering smoking cues may thus have elicited smoking-related thoughts and general curiosity about smoking in players, and this led to a smaller drop in temptation to smoke. In the culturally-incongruent condition, we hypothesise that players were less able to contextualise the game to their personal lives, and were, therefore, more likely not to be tempted by smoking cues in the game. In turn, this response corresponded to a greater drop in temptation to smoke. Potentially, then, culturally-relevant cue exposure is a factor designers should account for when designing tools to help people deal with cue-stimulated cravings. Cultural 
Temptation for smoking pre- and post-survey mean differences by version

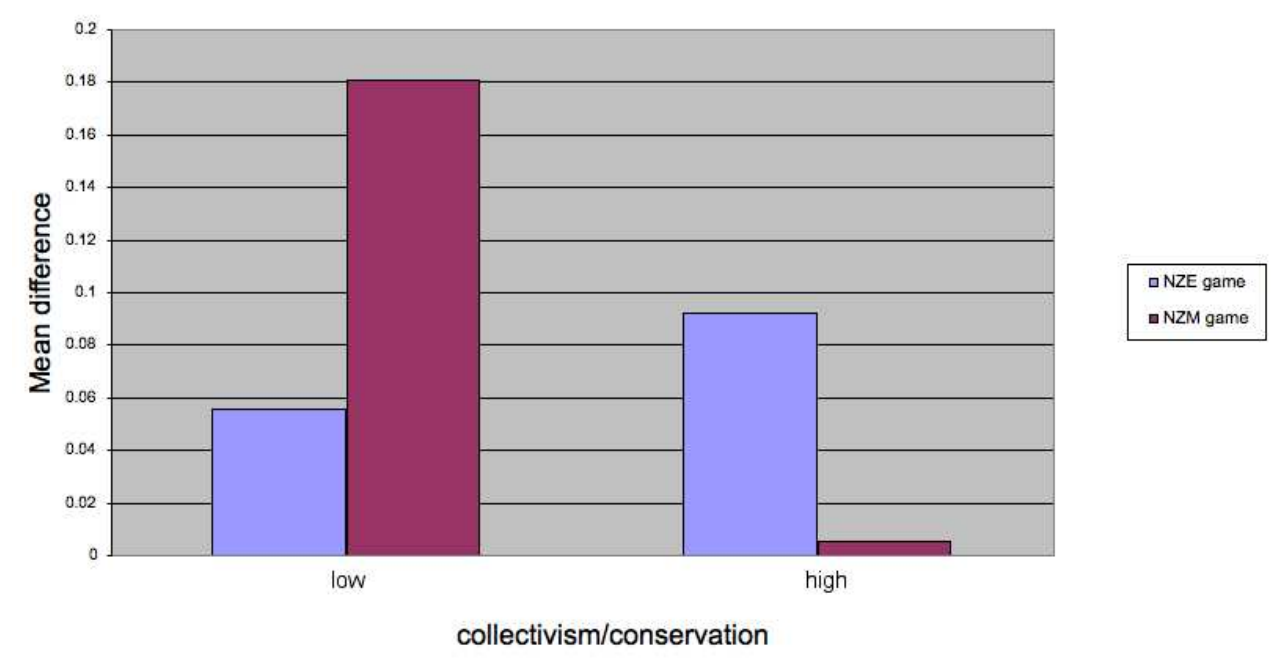

Figure 8.4: Changes in subjects' pre- and post- temptation to smoke by cultural orientation and game version

relevance may increase the potency of cue exposure effects.

As an alternative explanation, referencing the focus group findings we discussed in $\S 6.1$, the NZ European focus group members discussed how they viewed smoking as an activity with a more individual locus, while the Māori focus group members viewed it as having a more collective locus. In the culturally-incongruent conditions, smoking would have been portrayed in a more collectively-oriented manner for individualist players, and a more individually-oriented manner for collectivist players. Given the direct mismatch between the real life and game portrayals, smoking may have seemed more foreign and culturally-incongruent as an activity, and therefore less appealing. In turn, this may have led to greater increases in temptation reductions.

\subsubsection{Nonsignificant interactions between cultural orientation and game ver- sion}

We list here the regressions for which we did not find significant interactions:

- Post-survey means for temptation to smoke against status, proximity, pre-survey temptation to smoke, version, and individualism.

- Post-survey means for resistance to smoking against status, proximity, presurvey resistance to smoking, version, and collectivism.

- Post-survey means for intention to quit against status, proximity, pre-survey intention to quit, version, collectivism. 
- Post-survey means for positive beliefs against status, proximity, pre-survey positive beliefs, version, invidiualism.

- Post-survey means for positive beliefs against status, proximity, pre-survey positive beliefs, version, collectivism

- Post-survey means for negative beliefs against status, proximity, pre-survey negative beliefs, version, invidiualism

- Post-survey means for negative beliefs against status, proximity, pre-survey negative beliefs, version, collectivism.

\subsubsection{Discussion}

Overall, both the NZE and NZM versions of Smoke? were effective in decreasing players' positive beliefs about smoking and their temptation to smoke, while increasing their negative beliefs about smoking, intention to quit, and resistance to smoking.

Of the regressions with significant interactions, individualist players reported a greater change in intention to quit when playing the NZE rather than the NZM version, while collectivist players reported a greater change in intention to quit when playing the NZM rather than the NZE version. Both the individualist and collectivist players reported a greater change in resistance to smoking when playing the NZM version. Interestingly, in contrast to our expectations, the individualist players reported a greater reduction in temptation to smoke when playing the NZM version, while the collectivist players reported a greater reduction when playing the NZE version.

\subsection{The qualitative evaluation}

\subsubsection{Method}

Qualitative research methods have traditionally been employed in exploratory studies and in under-researched fields $[35,36]$. Our project fitted these criteria but, additionally, we were aware that the questions we had included in our quantitative survey could not identify specific cultural reasons for patterns of responses. Furthermore, the survey questions limited an investigation of whether the attitude shifts could be explained by players' appreciation of overt cultural references, or were related to more covert, deeply embedded cultural references in the game, by way of implementations of the design strategies. The survey 
responses also did not provide any insight into people's perceptions of the individual design strategies, so we could not examine how the strategies were being interpreted.

More generally, de Mooij suggests that evaluations of people's responses to persuasion that are premised on rational and linear thinking styles are inappropriate for use on collectivist audiences, because they are more characteristic of an individualistic worldview [38]. She suggests that evaluations of persuasion are more likely to elicit helpful information if they are focused on meaningfulness, not irritating message recipients, warmth, entertainment value, and aesthetic appeal [38].

To address these concerns, we ran some supplementary qualitative evaluations of both versions of Smoke? using the think-aloud protocol. We involved eight players, half were of NZ European origin (E1, E2, E3, and E4), and the other half were Māori (M1, M2, M3, and M4). Four of our players were assigned to play the NZE version of Smoke? (E1, E2, M1, and M2) and the other four were assigned to play the NZM version of Smoke? (E3, E4, M3, and M4). We discussed the think-aloud protocol, more details of our test participants, and the methodological specificities of this evaluation in $\$ 3.4 .2 .3$.

We have separated our discussion of the findings for each version of Smoke? into two sections. In $§ 8.2 .2$, we cover findings for the NZE version, and discuss players' perceptions of our use of SELF MONITORING, CONDITIONING, and TUNNELLING. In $\S 8.2 .3$, we discuss findings for the NZM version, and address players' perceptions of our use of MENTORING, GROUP OPINION, HARMONY, and TEAM PERFORMANCE. In both sections, we also discuss players' Identification with MC, Social responses, Wildcard activities, Perceived game effectiveness, and Game acceptance. We have structured the observations for players of each game in terms of the congruent condition, where the culture of the players matches the game's target audience, and the incongruent condition, where the culture of the players does not match the game's target audience.

\subsubsection{Findings for the NZE version}

\subsubsection{Overview of themes}

In table 8.6, we summarise the themed findings from the think-aloud sessions of E1 and E2 playing the NZE version of Smoke?, and in table 8.7 we present the equivalent findings for M1 and M2. 


\begin{tabular}{|l|l|}
\hline \multicolumn{2}{|c|}{ Think-aloud findings of E1 and E2 playing the NZE version of Smoke? } \\
\hline \hline Identification with MC & Representations of self. \\
\hline Self esteem & Very important for success. \\
\hline Game feedback & $\begin{array}{l}\text { Seeking positive feedback, avoiding negative feed- } \\
\text { back. }\end{array}$ \\
\hline Autonomy focus & $\begin{array}{l}\text { Welcoming, beliefs of importance of autonomy, irri- } \\
\text { tation at friends' peer pressure. }\end{array}$ \\
\hline Social responses & $\begin{array}{l}\text { High level of self-reliance, maintained relationships } \\
\text { to boost MC's mood, open to new relationships. }\end{array}$ \\
\hline Wildcard activities & Mostly solo activities, group activities for distraction. \\
\hline Perceived game effectiveness & $\begin{array}{l}\text { Effective as intervention tool, and not effective, in- } \\
\text { creased cravings. }\end{array}$ \\
\hline Game acceptance & Positive response towards game. \\
\hline
\end{tabular}

Table 8.6: Summary of findings for E1 and E2

\begin{tabular}{|l|l|}
\hline \multicolumn{2}{|c|}{ Think-aloud findings of M1 and M2 playing the NZE version of Smoke? } \\
\hline \hline Identification with MC & $\begin{array}{l}\text { Representation of self, representation of other group } \\
\text { member. }\end{array}$ \\
\hline Self esteem & Important for harmony with MC, not important. \\
\hline Game feedback & $\begin{array}{l}\text { Avoiding negative feedback, not concerned with } \\
\text { feedback. }\end{array}$ \\
\hline Autonomy focus & $\begin{array}{l}\text { Welcoming, but still acting to maintain group har- } \\
\text { mony. }\end{array}$ \\
\hline Social responses & $\begin{array}{l}\text { Reasonable level of self-reliance, less open to new } \\
\text { relationships, adopted avoidance tactics to preserve } \\
\text { harmony. }\end{array}$ \\
\hline Wildcard activities & Mostly solo activities. \\
\hline Perceived game effectiveness & Effective and captured real life events, not effective. \\
\hline Game acceptance & $\begin{array}{l}\text { Largely positive response, but with underlying ten- } \\
\text { sions. }\end{array}$ \\
\hline
\end{tabular}

Table 8.7: Summary of findings for M1 and M2

\subsubsection{Identification with MC}

In $\S 7.1$ we looked at how players' conceptions of their relationship with MC impacted on the way that they played. It is useful to comment on these conceptions at the outset, as they shaped our understanding of all the upcoming discussion themes.

The congruent condition: E1 and E2 E1 tended to view MC as a representation of herself. During the game set-up, E1 chose personas for MC that she thought were similar to herself. Later in the game, at decision making points, she justified her decisions in terms of her own preferred modes of conduct: 
I personally would [choose to call friends and organise an outing as a craving control activity], but she doesn't have very nice friends... so I think she should just have alone time to contemplate quitting (laughs).

E2 also talked about MC as a self-representation. For example, E2 used reasons based on her own behaviours for choosing MC's wildcard activities:

My self esteem would be low [after eating a tub of ice cream] because I'm a bit self-image conscious.

The incongruent condition: M1 and M2 Like E1 and E2, M2 also talked about $\mathrm{MC}$ in a way that indicated self-identification.

M1's identification with MC, on the other hand, would be better characterised as empathetic. During the game set-up, M1 chose personas for MC that she felt would be realistic for a current smoker (as M1 is a smoker herself). When she allowed MC to lapse, she felt sorry for MC, but was explicit that MC was separate from herself:

M1: Aww poor girl... sad.

Administrator: Why do you think it is sad?

M1: Because she gave in... well, I gave in really.

The conception of MC as a separate entity whose welfare M1 was concerned with is similar to the idea of ingroup members, which is characteristic of a collectivist worldview $[69,96,143]$.

\subsubsection{Self-esteem (application of SELF MONITORING)}

An important feature differentiating the NZE version of Smoke? from the NZM version is the presence of a self-esteem score. This is an application of the SELF MONITORING strategy. As we discussed in $\S 6.3$, self-esteem is considered to be an individualist construct [96].

The congruent condition: E1 and E2 Both of the NZ European players referred to the self-esteem score constantly throughout the game, and used it to guide their choices of action. For example, during a conversation with $\mathrm{MC}^{\prime}$ s mother in which MC's mother is somewhat sarcastic, E1 said:

This isn't a helpful conversation... it's pretty nasty... it's not really going to help, just make her feel worse about herself and go back to smoking. 
E1 seemed particularly focused on keeping MC in a good mood. On noting that Jake, MC's love interest, was having a negative impact on $\mathrm{MC}^{\prime}$ 's self-esteem, E1 said:

He's really mean to her, so I don't really like him that much... I don't like this boy, she should get rid of him.

E2 also talked about MC's self-esteem more than any other status field. For example, referring to the effects of Jake's words and actions on $\mathrm{MC}^{\prime}$ s state:

Jake's negative comment would decrease MC's self-esteem right now.

He misses her. That's going to boost her self-esteem.

The incongruent condition: M1 and M2 Like E1 and E2, M1 also used selfesteem as a factor in decision making. For example, M1 explained her choice not to add Jake as a control activity in the following way:

Calling him as a support is no good because he's so rigid towards her... okay, straight up, her self-esteem would go low... I feel sorry for her.

Interestingly, however, M1 seemed less focused on the goal of making MC completely smoke-free, because she allowed MC to lapse 14 times during two weeks:

...but look, she's cut down dramatically, she doesn't have to quit smoking... it's like crash dieting, you can't like, have lettuce all your life.

Based on the lack of concern with MC's quitting, we theorise that M1 was using self-esteem less as a means to success, and more as a general barometer of how happy she was able to make MC feel. Because M1 did not conceptualise MC as herself, perhaps by keeping $M C^{\prime}$ 's self-esteem high, she felt that she was helping to maintain ingroup happiness levels, with $\mathrm{MC}$ as part of her ingroup.

Unlike all the other players of the NZE version of Smoke?, M2 did not discuss self-esteem or refer to its presence at all throughout the game.

\subsubsection{Game feedback (application of CONDITIONING)}

Related to the drive to increase self-esteem is the seeking of positive feedback, which is more acceptable than negative feedback in individualist cultures [96, 142]. In the NZE version, then, we tried to minimise negative game feedback. 
The congruent condition: E1 and E2 E1 and E2 both believed that obtaining positive feedback would be instrumental in keeping MC on track with quitting. For example, E1 illustrated a belief that it was important to keep self-esteem boosted, even at the cost of personal relationships, in this comment about MC's love interest:

I don't think she should talk to her boyfriend because he gives her negative feedback.

On encountering Hayden, a non-smoker character in the game, E1 said:

Look, this guy's good because he's already quit... that guy's really positive, maybe she should hang out with him and get buddies.

E1 voiced how she was using $\mathrm{MC}^{\prime}$ s happiness as a short-term goal aiding the larger goal of complete cessation:

...smoking produces endorphins, and so if she plays netball she'll naturally produce endorphins.

E2 chose activities for $\mathrm{MC}$ that she believed would avoid negative feedback. For example, she made MC go for a run (alone), and avoid a gym class because she thought that running with a friend might decrease $M C^{\prime}$ 's self-esteem, as MC was not fit. E2 also avoided cooking as a wildcard activity, because she thought it might lead to over-eating which, again, could have resulted in a drop in self-esteem.

The incongruent condition: M1 and M2 Like E1 and E2, M1 appeared to choose actions for MC based on what she thought would keep MC in a positive mental state. For example, she explained why she chose to have MC avoid her love interest at a club:

I don't want him to kill her night cos he's always so nazi to her.

In contrast to the others, M2 made no overt reference to the importance of keeping $\mathrm{MC}$ feeling positive. He focused rather on doing whatever he thought was necessary to keep MC from smoking. For example, he chose to make MC talk to a stranger at a club after MC had a fight with his love interest, because he noted that the fight had increased MC's temptation to smoke. Similarly, M2 tended to make MC listen to music as a control activity because it is what he would do himself in real life to soothe frustration. 


\subsubsection{Autonomy focus (application of TUNNELLING)}

We have tried to convey in the NZE version of Smoke? a sense of autonomy over the quitting process, particularly in terms of reliance on other people. The autonomy focus of the NZE version, in fact, serves as an application of the TUNNELLING strategy.

The congruent condition: E1 and E2 E1 and E2 showed that they subscribed to a personal view of control over the quitting process. This is what we would expect because individualists have a more autonomous sense of control over their lives $[59,69,96,143]$. For example E2 stated:

...[quitting smoking is] definitely something you do on your own, no matter how much support you get, it's up to you.

At the same time, E1 said that MC's friends needed to "stop pressuring her", so she chose to make MC confront them about their lack of support, explaining that she wanted her friends to understand $\mathrm{MC}^{\prime}$ s position and take more responsibility in helping her quit. It is interesting to reflect here on E1's expectations of MC's friends because the idea of needing social support may seem like a collectivistic expectation. Collectivists would be unlikely to adopt such an approach, however, as they would tend to minimise conflict and would not engage in confrontations for fear of disrupting group harmony [59, 96, 143].

E2, in contrast, was untroubled by MC's friends not supporting her. Her preference for completely independent decision making is indicated by this comment about peer pressure:

MC should still have an independent decision... it's not up to $M C^{\prime}$ 's friend to make the decision.

The incongruent condition: M1 and M2 At one point in the game, M1 chose to have MC make up with her best friend. In justifying this choice, she said:

It's not her friend's responsibility. If MC wants to quit she has to do it on her own accord, and part of quitting, you know... of course there will be influential factors around you, so she just has to have the will power, it's like dieting. There's going to be people eating junk food around you, you just have to say no.

We can read M1's response in at least two ways. At one level, she is explicitly stating that MC needs to retain personal control over her choice, and that MC 
should not need to rely on others. This is a more individualistic tendency. At the same time, she chose to make up with the friend, even though she did not have to, and we can read this as a desire to minimise confrontation with other group members, exhibiting a collectivistic tendency [59, 96, 143].

M2's attitude towards MC's friends was similarly mixed. M2 claimed early on in the game that friends are important in helping MC quit because friends can talk MC through the process. At the same time, when $\mathrm{MC}^{\prime}$ s friends offered $\mathrm{MC}$ a cigarette, M2 directly chose not to accept it. Yet later, when the same friend did not turn up to meet MC, M2 had the option to confront the friend about it, but chose not to, stating that confrontation was unnecessary.

\subsubsection{Social responses}

An aspect of how participants played the NZE version of Smoke? that may be revealing of cultural tendencies is their way of handling $\mathrm{MC}^{\prime}$ 's social relationships. When a scene contains NPCs that MC can interact with, players decide whether $\mathrm{MC}$ talks to them or avoids them. Additionally, most of the interaction decision points present players with three potential responses for $\mathrm{MC}$, from which they must choose one response. One of the responses generally supports what the NPC has just said, another tends to be neutral, or in slight disagreement with the NPC, and the third can be characterised as strong disagreement.

The congruent condition: E1 and E2 E2's actions and justifications given during the game indicated that she was dubious about whether other NPCs would support MC. She justified choosing solo activities because she was concerned that MC would feel inferior to others. E2 also talked about how MC was susceptible to peer pressure. She did, however, express her belief that it was important for $\mathrm{MC}$ to have the support of his mother in quitting:

... if the mum didn't support MC by saying supportive comments, MC wouldn't feel capable of quitting.

Additionally, E2 seemed open to interacting with characters that MC did not have prior relationships with (e.g. Hayden). Overall, E2's style of socialising in the game falls within typical individualist patterns, i.e. an awareness of competition, a feeling of connection to close family, and a willingness to engage with outgroup members.

E1 seemed more socially-oriented in her actions than E2, but generally seemed to have a specific agenda in mind: that of keeping MC in a happy mood. She was willing to break ties with characters who she thought were not keeping MC 
happy (e.g. MC's love interest), and when she eventually chose to talk to MC's friends about not being supportive, she chose the "you should have been more supportive" line of response. Her reason for doing so was so that MC could explain to the friends how they needed to help her quit. So while E1 sought social company, it was less for the sake of reinforcing ingroup memberships (a more collectivistic motivation) and more for personal enhancement reasons.

The incongruent condition: M1 and M2 Unlike E1 and E2, M2 was less willing to talk to random characters, but placed a lot of importance on how much support $\mathrm{MC}^{\prime}$ s love interest could provide. M2 directly turned down any smoking offers, but avoided confrontation at all other times.

M1 frequently vocalised her dislike of MC's love interest, but adopted avoidance tactics rather than confrontation to minimise contact with him. In addition, at the end of the game, she chose to have MC make amends with the best friend, even though it was not necessary. So both M1 and M2 were similar in their drives to maintain harmony and avoid conflict, a more collectivistic trait $[59,69,143]$, but were obliged to balance this tendency with $M C^{\prime}$ 's quitting objective. In the NZE version this involved outright disagreement with at least one important character.

\subsubsection{Wildcard activities}

Between plot development scenes, players are faced with wildcard scenes, in which they are required to choose wildcard activities which have a random outcome on MC's mood.

The congruent condition: E1 and E2 E1 picked a selection of solo and sociallyoriented activities. Her reasons for picking social activities, however, were less based on bonding with others, and more based on how they would make MC feel personally. This is in line with more individualistic tendencies [59, 69, 96, 143]. For example, she explained how she was going about keeping MC distracted from cravings:

...I think she needs to interact with other people to keep her happy.

Another reason why we believe E1 was using socially-oriented wildcard activities as a distraction was because she was ready to jettison existing social relationships. This is not a path a collectivist would take unless absolutely necessary [59, 96, 143]:

He's really mean to her... I don't like this boy, she should get rid of him. 
E2 mostly chose solo wildcard activities for MC. She made MC run alone, clean her room, work on an essay, and read blogs. E2 did, however, make MC engage in a social outing (bowling) but, again, gave distraction as an underlying reason, rather than the importance of social relationships:

...because that's an ongoing thing. It keeps you occupied and just engaged in a lot of distracting activity and there's a fun goal at the end of it. Bowling is also a form of exercising.

The incongruent condition: M1 and M2 M1 appeared to favour activities that would be beneficial for MC alone, such as running:

M1: It's really got to be something she's got to do, you know?

Administrator: Herself?

M1: Something she enjoys doing... yeah.

This would appear to be more indicative of an individualistic worldview. At the same time, M1's justifications for actions seemed malleable to suggestion. For example, later in the game when MC explicitly seeks social support, M1 said:

Social support, that's what you need, eh! That's why I got her friend to go with her [to lunch].

M2 also, unexpectedly, seemed to favour individual activities, such as paid work, running, getting a massage, writing an essay, shopping, and weight training. Yet, during retrospective questioning, M2 stated that his own relatives acted differently when trying to quit smoking:

...my relatives turn to the whanau to quit smoking.

\subsubsection{Perceived game effectiveness}

The congruent condition: E1 and E2 E1 and E2 had different opinions on the effectiveness of the game.

E1 said that the game had not persuaded her to quit smoking, and that the frequent smoking cue exposure had increased her own cravings for a cigarette.

E2 was more positive about the game, and thought that it might be a good tool for people to use during the quitting process. She thought that it was effective because it communicated MC's health changes mostly through changes in status. Explaining her views on the best way to persuade people:

Instead of telling people to not smoke, the better way to make them stop is to let them make their own decision. 
E2 noted that MC's mother had used such an approach during conversation. Reliance on one's own judgement is more common in individualist, than in collectivist cultures $[69,96,142]$.

The incongruent condition: M1 and M2 As with E1 and E2, M1 and M2 differed in their opinions of the effectiveness of the game.

Like E1, M1 stated that she did not feel that the game had convinced her to quit smoking.

In contrast, M2 spoke positively about the game. Unlike E2, however, he thought that the power of the game lay in people's ability to relate to MC. M2 went on to say:

...the game captured real-life events and statements.

\subsubsection{Game acceptance}

The congruent condition: E1 and E2 Both E1 and E2 seemed to understand all the concepts represented in the game content and narrative. While E1 did complain about the unhelpful nature of MC's social interactions, both she and E2 demonstrated in their choices that they gave preference to individual motives for action. At the very least, neither E1 or E2 seemed surprised by anything they encountered. This seems to reflect Hall's notion of the "hidden program" [62], by which people readily accept what they encounter if it is culturally familiar.

The incongruent condition: M1 and M2 Most of what M1 and M2 vocalised seemed to indicate that they had similar independence-oriented motivations as $\mathrm{E} 1$ and E2. One way to reason about this interesting result is the effects of acculturation: both M1 and M2 had spent much of their lives in NZ European-dominated systems, and thus might have become adept at moving between Māori and NZ European identities. Another possible reason related to the "multiple identities" perspective is what Hong et al. term frame-switching [160]. This is the switching between cultural knowledge structures, choosing the one that seems more relevant and readily-available, if knowledge exists for more than one structure. Yet another factor potentially affecting the responses of M1 and M2 is the phenomenon termed "socially desirable responding", where people tend not to tell complete truths to save face, a behaviour which people of collectivistic orientations are more likely to engage in [142]. Additionally, Vatrapu and Quinones found that when the ethnic background of usability evaluators matches the background of participants, evaluators are more successful in eliciting feedback [147]. 
The evaluator of M1 and M2 was clearly of European descent, and therefore not matched to their backgrounds.

Supporting all of these potential reasons is the observation that, despite the verbal explanations proffered by M1 and M2, some of their actions, such as their avoidance of conflict, seemed to indicate the existence of potentially different motivations that they were not expressing.

Summary E1 and E2 accepted much of the content of the NZE version of Smoke?, whereas M1 and M2 accepted many, but not all, aspects of the NZE version.

\subsubsection{Findings for the NZM version}

\subsubsection{Overview of themes}

We summarise the themes of the think-aloud sessions of M3 and M4 playing the NZM version of Smoke? in table 8.8, and those of E3 and E4 in 8.9.

\begin{tabular}{|l|l|}
\hline \multicolumn{2}{|c|}{ Think-aloud findings of M3 and M4 playing the NZM version of Smoke? } \\
\hline Identification with MC & Self representations. \\
\hline Respect & Very important for success. \\
\hline Jade status & $\begin{array}{l}\text { Initially welcoming but later forgotten, welcoming } \\
\text { and not forgotten. }\end{array}$ \\
\hline Moods + opinions & Checked and used moods and opinions. \\
\hline Sandwich + tag minigames & Minigame challenges taken seriously. \\
\hline Social density & $\begin{array}{l}\text { Acceptance, welcoming of social support, acted to } \\
\text { preserve harmony. }\end{array}$ \\
\hline Opinions from elders & Consulted with elders, found opinions useful. \\
\hline Convince best friend & Frustrated by challenge. \\
\hline Social responses & $\begin{array}{l}\text { Found group presence helpful, viewed smoking } \\
\text { with social locus, acted to preserve harmony. }\end{array}$ \\
\hline Wildcard activities & Socially-oriented activities. \\
\hline Perceived game effectiveness & Effective as prevention tool, engaging, and fun. \\
\hline Game acceptance & $\begin{array}{l}\text { Positive response towards game, representative of } \\
\text { quitting in Māori culture. }\end{array}$ \\
\hline
\end{tabular}

Table 8.8: Summary of findings for M3 and M4

\subsubsection{Identification with MC}

The congruent condition: M3 and M4 M3 maintained a self-identification view of his relationship with MC. For example, during the set-up phase, M3 explained how he was choosing $\mathrm{MC}^{\prime}$ s personas: 


\begin{tabular}{|l|l|}
\hline \multicolumn{2}{|c|}{ Think-aloud findings E3 and E4 playing the NZM version of Smoke? } \\
\hline Identification with MC & $\begin{array}{l}\text { Self representation becoming other representation, } \\
\text { other representation. }\end{array}$ \\
\hline Respect & Not important for success. \\
\hline Jade status & $\begin{array}{l}\text { Surprised, and later forgotten, irritated by responsi- } \\
\text { bility. }\end{array}$ \\
\hline Moods + opinions & Did not check or use moods and opinions. \\
\hline Sandwich + tag minigames & Irritated by social nature of minigames. \\
\hline Social density & Distracted by social density. \\
\hline Opinions from elders & $\begin{array}{l}\text { Did not consult unless necessary, found attitudes ir- } \\
\text { ritating. }\end{array}$ \\
\hline Convince best friend & Enjoyed challenge, thought it was clever. \\
\hline Social responses & $\begin{array}{l}\text { Group presence not helpful, viewed smoking with } \\
\text { individual locus. }\end{array}$ \\
\hline Wildcard activities & Solo activities. \\
\hline Perceived game effectiveness & Effective as prevention tool, engaging, and fun. \\
\hline Game acceptance & Average to negative response to game. \\
\hline
\end{tabular}

Table 8.9: Summary of findings for E3 and E4

Later in the game, he related instances in $M C^{\prime}$ s life to his own:

This reminds me of a friend of mine who quit drugs. This kind of conversation happened with him as well... it wasn't helpful.

M4 also clearly identified with MC, and said that the way she played the game came "natural" to her. Commenting on a particular part of the game, M4 said the following, illustrating a strong level of cultural identification with the game content:

This always happens to us Māoris.

The incongruent condition: E3 and E4 E3 initially identified MC as a representation of himself. As the game progressed, however, E3 pointed out that he was aware that he was not an intended target audience for the game:

E3: I'm assuming this is targeted towards Māori or Polynesians.

Administrator: What cues give you that idea?

E3: I guess the language

Administrator: Anything else?

E3: The family and stuff.

E4 openly said that he did not identify, and moreover, did not want to identify with MC, and claimed that he was just acting as a guide for MC. 


\subsubsection{Respect (application of MENTORING)}

A defining feature of the NZM version of Smoke? is the respect score, which serves as an application of our MENTORING guideline. With the respect score, we aimed to communicate a sense of group responsibility, which is of central importance in collectivist cultures [69, 96, 142, 143].

The congruent condition: M3 and M4 M3 said the following on noticing the presence of the respect score in the game:

Oh is he Māori? Because for Māori people this would be very important. Its like collective responsibility, you know? When one person does something everyone feels responsible, that's what it makes me think anyway.

M3 continued to check the respect score for the remainder of the game.

While M4 did not comment directly on the respect score, she did state that she thought that MC's quitting success depended on how he was perceived by the whanau (family) and others, and not just by his own standards.

The incongruent condition: E3 and E4 In contrast to M3 and M4, E3 viewed the status screen and commented:

General health... Whanau respect... Cigarettes smoked... is respect really that important?

Neither E3 nor E4 overtly referred to the respect score during the game.

\subsubsection{Jade status (application of MENTORING)}

Jade's presence, and particularly her status screen, is an application of the MENTORING strategy in the NZM version of Smoke?

The congruent condition: M3 and M4 M4 found Jade's presence to be motivational and inspirational, and commented that what MC did had an affect on everyone, including Jade. She also illustrated a collectivist perspective on the interconnectedness of role-modelling and acting to serve the interests of the ingroup, by commenting about the opinions of the family [69, 96, 142]:

They are happy with his progress so he can keep on setting a good example for Jade.

M3 initially responded positively to Jade's presence:

Oh she's so cute!

For the remainder of the game, however, M3 neglected to check Jade's status. 
The incongruent condition: E3 and E4 In complete contrast, neither E3 nor E4 found Jade's presence to be pleasant, useful, or motivational. On encountering a reference to Jade in the game, E3 said:

Who the hell is Jade?

Later on in the game, he said:

It didn't cross my mind to check Jade's status, I don't think anything I would do would change her status. I forgot about her.

E4 voiced his irritation at Jade's presence:

Surely when you crave a cigarette you want to just be alone and also keep doing things like touch [rugby] that can distract you and not look after kids and stuff.

\subsubsection{Moods + opinions box (application of GROUP OPINION and HARMONY)}

The moods + opinions box is another unique element of the NZM version of Smoke? representing applications of the GROUP OPINION and HARMONY strategies. A common feature of collectivist culture is a consensus-oriented way of decision making $[38,59,142]$.

The congruent condition: M3 and M4 Both M3 and M4 made use of the moods + opinions box throughout the game. M4 said the following about the presence of other family members:

They give some positive feedback and can encourage you.

The incongruent condition: E3 and E4 E3, on the other hand, did not check the opinions at all, and when he was asked whether he was intending to check them answered:

Nah, not really, only if I think they are useful.

E4 did not check the opinions either, and commented about decision making: MC can decide what he wants to do.

Both E3 and E4 clearly preferred a more individualistic process of decision making. 


\subsubsection{Sandwich + tag game (application of HARMONY)}

The sandwich game and tag game are the minigames featured in the NZM version of Smoke?, and act as applications of the HARMONY guideline.

The congruent condition: M3 and M4 M3 readily accepted the sandwich making task, and even become flustered by his performance:

Oh no, I can't even read it, oh I'm so bad at this... rye, rye, mayo, lettuce, was there lettuce? Tomato, I think there was tomato, oh my gosh, next person...

It seemed that M3 had taken the responsibility of making sandwiches for other family members willingly, and did not want to disappoint them. This is a classically collectivistic trait $[51,59,142,162]$.

The incongruent condition: E3 and E4 Both E3 and E4 specifically vocalised their dislike of the social nature of the sandwich game:

Why do I have to make the sandwiches? That's sucks... stupid game!

Why does he need to make sandwiches for everyone, he can just grab lunch on his own!

\subsubsection{Social density (application of HARMONY)}

General social density is a theme of the NZM version of Smoke? representing an application of the HARMONY guideline.

The congruent condition: M3 and M4 Neither M3 nor M4 found the constant presence of many NPCs to be particularly unusual. M4 in fact described their presence in the game as a built-in support system that made players aware of themselves and their relationships to others. She also said:

The family members make MC feel safe and less judged.

...family understand how difficult the quitting process can be and they will always be there to support you if you want to try and quit.

When he had to select a response for MC, M3 illustrated his own drive to avoid conflict and maintain harmony, and said:

I'm gonna pick this option as the others are quite defensive even if it's not true.

The constant presence of extended family is common in collectivist cultures [38, $69,142,143]$. 
The incongruent condition: E3 and E4 Unlike the Māori players, E3 and E4 found the presence of family members and other characters distracting. E4 said the following about family:

They can sometimes discourage and tease you and say that 'old habits die hard' and that 'it's not that easy to quit' or make comments and stuff like 'I wonder how long he will stay quit?'

On succeeding in making MC remain smoke-free for a year, E4 also illustrated his belief that quitting had an individual locus of control, and that other family members had had little, if any, impact on MC's success:

\section{MC did it - now he can go celebrate!}

\subsubsection{Opinions from elders (application of GROUP OPINION)}

Another application of the GROUP OPINION guideline in the NZM version of Smoke? is the presentation of opinions from elder family members, because elder family members have a privileged position in collectivist cultures [69, 142].

The congruent condition: M3 and M4 In general, both M3 and M4 found the opinions presented to be helpful in guiding their decisions for MC. With respect to the grandmother's wishes that MC repair his damaged relationship with his cousin, M3 rationalised the grandmother's attitude, saying the following:

That's just normal, isn't it? I imagine my gran would talk to both of us.

The incongruent condition: E3 and E4 In contrast, E3 verbalised the unfairness of the grandmother's opinion while still choosing to resolve issues with the cousin. When questioned why he made MC take this course of action, E3 responded with the following:

I suppose it's the right thing to do.

In this case, E3 demonstrated a reliance on universal norms for guiding behaviour, compared to ingroup norms, which is more typical of individualists than collectivists $[96,143]$. 


\subsubsection{Convince best friend (application of TEAM PERFORMANCE)}

The TEAM PERFORMANCE guideline is implemented in the NZM version of Smoke? in the form of the convince best friend challenge. The key objective is to find the correct combination of arguments that will convince $\mathrm{MC}^{\prime}$ 's best friend to consider quitting with MC. We expected that collectivist players would be motivated to search for the correct combination, on the assumption that the best friend's wellbeing would be impact on players' views of $\mathrm{MC}^{\prime}$ s well-being.

The congruent condition: M3 and M4 After trying several arguments on the best friend unsuccessfully, M3 began to get frustrated that his choices of arguments were not proving successful. Referring to arguments as "explanations", he vocalised his frustration in comments such as:

\section{Oh why doesn't this explanation work?}

M3 might have assumed that what he himself found persuasive should have worked on $\mathrm{MC}^{\prime}$ s best friend. In fact, this belief may be explained by the tendency of members of collectivist ingroups to overtly share a set of preferences and attitudes $[59,69,96,142,143]$.

The incongruent condition: E3 and E4 In contrast, E3 voiced how he liked the convince best friend challenge, but for reasons related to personal customisation, which we had not considered:

This is quite clever, it's good to show different people have different reasons for making decisions such as giving up smoking, it's not the same for everyone.

So although we implemented the convince best friend challenge to appeal to players with collectivist values, it clearly communicated individualist appeals as well. This breadth of appeal made it a sub-optimal implementation of the TEAM PERFORMANCE guideline.

\subsubsection{Social responses}

The congruent condition: M3 and M4 M3 took advantage of all family socialisation opportunities, as we would expect from a player of a collectivist culture. He consistently consulted the moods + opinions box, almost always chose sociallyoriented wildcard activities for MC, and had MC interact with all of his family members. On being presented with options for whether MC should go to hospital to see his grandmother, which he did decide in favour of, he said: 
It's not even a choice! Does he go to the hospital with his family — are you serious??

M3 also indicated that he was accustomed to harmony preservation, a defining feature of collectivism $[59,69,143]$, when he paralleled the wishes of MC's grandmother with how his own grandmother would behave.

M4, like M3, was keen to have MC make use of all family support. She made MC talk to as many people as possible about quitting, explaining that it was an important reflective and motivational step in the quitting process. M4, in particular, was cautious about MC's actions in relation to Jade, emphasising a concern for role-modelling.

The incongruent condition: E3 and E4 E3 and E4 were less enthused about the constant presence of MC's extended family members which is not a common feature of individualist cultures $[38,69,142,143]$. Additionally, they were perplexed by the frequent need to look after, and take responsibility for other characters. E3, in fact, sided with MC's best friend, when the best friend did not turn up for an arranged dinner engagement with MC:

I've had that before, you know, if there's someone who's doing something you don't want to do, you avoid them.

Demonstrating a more individualist mentality, E4 stated that he believed that quitting was best achieved individually. This belief was partially borne out by his choice to rely solely on phoning MC's love interest as a social support control activity, and to decline other channels of social support.

\subsubsection{Wildcard activities}

The congruent condition: M3 and M4 With regards to choosing activities for MC during wildcard scenes, both M3 and M4 largely selected socially-oriented activities. This demonstrates a typically collectivistic tendency to prefer be in the company of others $[38,51,96,143]$. For example, M4 chose to have MC play with Jade, go for walks with his mother, and play netball. M3 also chose social activities, and then expressed dismay when some of them triggered MC to feel worse:

Oh no! I thought it would be fun, you know, to be around everyone? I guess things don't always work out how you expect. 
The incongruent condition: E3 and E4 In direct contrast, both E3 and E4 preferred to select solo activities. E3 made MC take a stroll by himself to the beach, complete sudoku puzzles, and do homework, while E4 chose to make MC work out, and play the guitar.

\subsubsection{Perceived game effectiveness}

Unlike the NZE version of Smoke?, all players of the NZM version thought that it could be used as an effective tool in smoke-free promotion.

The congruent condition: M3 and M4 While M3 thought that the game was effective, he stated that he thought it would be better suited as a prevention, rather than intervention tool, as a way to highlight to non-smokers the consequences and difficulties of quitting.

M4 said that she had found the game engaging and fun. She particularly enjoyed the social interaction between characters, and reported she was "not bored with renditions of the same old stuff, as information was given in subtle, different, creative and non-threatening ways". She went on to say:

As a Māori who smokes, this game has given me some things to think about. It is nothing I didn't know, it is just that again I have realized how much better it would be if I stopped.

The incongruent condition: E3 and E4 Like M3, E3 thought that the game would be most effective as a prevention tool.

Despite his inability to identify with MC, E4 said that he had found the game engaging, and considered it to be "good". At the same time, he voiced his concern that after a few sessions of play, he would begin to exhaust the range of new information in the game. His opinion was that such a game would only be useful as a brief intervention.

\subsubsection{Game acceptance}

The congruent condition: M3 and M4 M3 and M4 largely responded in accordance with our design intentions for the NZM version of Smoke? Apart from M3's lack of interest in Jade's welfare, M3 and M4's verbalisations during play highlighted that they understood the group-oriented motivations underlying the NZM version-specific elements of the interface and narrative. They specifically used the group-oriented interface elements during play, and factored group motivations into their interpretation of the narrative. In questioning after the game, 
M4 even said that events in the game reminded her of events from her own life, and that the game was "the way it is in Māori culture".

The incongruent condition: E3 and E4 In direct contrast, neither E3 nor E4 particularly related to the cultural assumptions underlying the NZM version of Smoke? They were confused by the group-centric interface elements and narrative details, and on occasion explicitly stated that they viewed issues of cessation to be more individual than group-focused. Indeed, both E3 and E4 became annoyed with the group emphasis built into the NZM version.

It is interesting to reflect on the differences in reaction between the NZ European players of the NZM version, and the Māori players of the NZE version. A reason for the differences in reaction valence might be explained by the effects of majority and minority audience targeting. Minority audiences are accustomed to not being the intended recipients of much communication material, and are familiar with majority audience-targeted material. Aaker et al. found that when minority audiences encounter material targeted at them, they tend to respond to it more positively than majority audience members respond to material targeted at them [3]. In contrast, majority audience members respond more negatively to material targeted at minorities than minority audiences responses to majoritytargeted material. E3 and E4's lack of acceptance of the NZM version is potentially explained by an absence of acculturation to Māori culture, because it is less accessible to the general public than the dominant, NZ European representations of culture.

Summary M3 and M4 mostly accepted and welcomed the content of the NZM version of Smoke?, whereas E3 and E4 largely did not.

\subsection{Discussion}

\subsubsection{Support for our hypothesis}

In $§ 3.1 .1$, we presented our hypothesis: persuasive technology is more effective when it reflects the culture of its intended target audience.

The quantitative evaluations of Smoke? provided us with preliminary evidence supporting our hypothesis, particularly when we looked at attitude changes relating to subjects' intention to quit, and also their resistance to smoking. We did, however, find that attitude changes relating to temptation to smoke contradicted what we had initially expected, although we could accommodate these unexpected shifts within our research perspective. Importantly, both versions of the 
game were shown to be effective in increasing various kinds of anti-smoking beliefs, regardless of players' cultural orientations. While the quantitative analysis allowed us to investigate effects on players' attitudes resulting from the game as complete artefacts, it gave us no insight into how players were reacting to our use of specific design strategies, or their reasoning for adopting particular courses of action.

The qualitative evaluation allowed us to investigate more closely players' immediate reactions to, and interpretations of Smoke? The players explicitly referenced notions of culture in their verbalisation, so we were able to definitively relate their impressions of the game to culture. We were also able to note where players explicitly interacted with guideline-informed game interface elements, which allowed us to rule out basic appreciation of overt cultural references as a main reason for attitude change. Like the quantitative analysis, the findings of the qualitative evaluation also supported our hypothesis. Players had more positive responses to the game version that reflected their cultural background, and had either mixed, or more negative responses to the game version that did not reflect their cultural background.

A limitation of our evaluation is that the quantitative, and qualitative test participants may not have been representative of typical members of individualist and collectivist cultures. Additionally, our participants were an amalgam of smokers, ex-smokers, and non-smokers: it is possible that the responses and interpretations of smokers are quite different to those of non-smokers. Furthermore, Smoke? was designed to be played more than once, but participants involved in our evaluations played it just once. As such, their responses reflect a singleexposure to the game. We believe that playing the game more than once would have led to a greater attitude change in players.

\subsubsection{Assessment of the design strategies}

Both the NZE and NZM versions of Smoke? were informed by different combinations of PT design strategies. While the quantitative evaluation did not allow us to isolate and assess the effectiveness of any one individual guideline, the qualitative analysis provided us with a much better insight into players' responses to the strategies. The qualitative insight, however, was specific to our prototype, as the players were reacting to our Smoke?-specific implementations of the strategies, and not the strategies themselves.

Working within these constraints, the qualitative evaluation participants overall perceived and interpreted the culturally-relevant PT design strategies implemented in the game as we had hoped, and the Māori players even used many 
of the guideline-based game features to inform and enhance their playing of the NZM version of Smoke? There was one notable instance of a Māori player not responding to a game application of the TEAM PERFORMANCE strategies as we had intended: he became frustrated by the convince best friend challenge. We cannot determine whether this was due to the guideline being ineffective, or our convince best friend implementation of TEAM PERFORMANCE not bearing close enough resemblance to the abstract guideline.

Clearly, the implementation of culturally-relevant PT design strategies in a technology plays a central role in the effectiveness of the resulting technology. Had we chosen to implement the strategies differently, almost certainly we would have reported different outcomes. As Smoke? represents the first application of the design strategies, we are not in a position to comment on how well we captured their essence in our design. The question of how best to implement the strategies is one that can only be answered with further investigation, and this is an important next step. Optimal implementations of the design strategies are more likely to yield more effective culturally-relevant persuasive technology. 


\section{Chapter 9}

\section{Conclusions}

Persuasion concerns changing peoples' attitudes and behaviours, many of which are influenced by culture $[4,38,62,143]$. Harnessing the dominant motivations typically underlying people's actions, both in an individual and social context, should lead to greater persuasion. This concept has already been successfully applied in the domain of cross-cultural consumer advertising (e.g. $[4,38,61])$. A similar approach should be adopted for persuasive technology. In harnessing culture at design time, we are more likely to produce more sensitive, realistic, and effective PT tools.

Chapter 2: Background We began this thesis by examining the key concepts of persuasive technology and cultural dimensions, and looked for regions of cross-over between these areas and the fields of culturally-focused $\mathrm{HCI}$ and cross-cultural consumer behaviour. We showed that as yet, there are few intersections between these bodies of literature. In fact, as yet, no studies have focused on how to design culturally-relevant persuasive technology.

Chapter 3: Methodology We then provided a theoretical justification for our research focus, and proposed the following hypothesis:

Persuasive technology is more effective when it reflects the culture of its intended target audience.

Investigating this hypothesis, our study was comprised of three research phases: the theory development phase, the prototype development phase, and finally, the evaluation phase. We also discussed the details of our use of methods for every phase.

Chapter 4: Theory For the theory development phase, we interviewed stakeholders of NZ social marketing organisations, to determine whether culturallyrelevant persuasive approaches played a part in the organisations' cam- 
paigns. Next, we examined the motivations underlying Fogg's PT strategies, using as a context the cross-cultural psychology literature on motivations of individualists and collectivists. Combining some of these findings with other observations from the cross-cultural psychology literature, we presented a set of original culturally-relevant PT design strategies.

Chapter 5: Smoke? Game mechanics In our discussion of the first stage of the prototype development phase, we presented the rationale underlying the game mechanics of our culturally-relevant PT game Smoke? We also overviewed the key components of Smoke? and discussed common narrative features between the two versions of the game.

Chapter 6: Smoke? Incorporating culture We shared findings from focus groups we carried out with NZ European and Māori participants regarding smokingrelated beliefs. We then showed how we applied the focus group findings to the game design. We explained both how the findings informed game content, and also how they determined which strategies we used in the design of the two game versions and the form of our strategy applications.

Chapter 7: Smoke? Play testing and refinement In our discussion of the second stage of the prototype development phase, we summarised the findings of play tests of both versions of Smoke? These took the form of a user evaluation as well as an expert evaluation of the game. For the user evaluation, we focused on explaining problems identified by the users during play by relating features of our game to PT theory, including Fogg's strategies and our own culturally-relevant design strategies. For the expert evaluation, we concentrated purely on usability issues found in the game by the team of experts. Alongside the different problems identified during the play testing, we showed how we implemented our proposed solutions in the final versions of Smoke?

Chapter 8: Evaluation For the evaluation phase, we presented the results of separate quantitative and qualitative evaluations of NZ European and Māori players' experiences of the NZE and NZM versions of Smoke? The results of the quantitative evaluation of Smoke? largely indicated that when subjects played a game version that reflected their cultural orientation, they demonstrated greater changes in anti-smoking attitudes than when they played a version that did not reflect their cultural orientation. Additionally, overall, both game versions increased anti-smoking attitudes in all players. The qualitative evaluation also showed that cultural relevance was an important 
factor in players' experiences of the game. Many of the interface components informed by our culturally-relevant design strategies were positively received by, and used by our target players, while being misunderstood by, and underused by non-target players. In summary, both the quantitative and qualitative evaluations of the two versions of Smoke? confirmed our research hypothesis.

\subsection{Contributions}

In carrying out this research, we have made three contributions to the PT literature.

\subsubsection{An original set of culturally-relevant PT design strategies}

We developed a set of culturally-relevant PT design strategies, mostly based on existing cross-cultural psychology literature on individualism and collectivism. Our strategies were also informed by an examination of the cultural motivations harnessed in Fogg's PT strategies, and findings from interviews we conducted with stakeholders of NZ social marketing organisations.

Our strategies are contextualised in the cultural dimensions of individualism and collectivism. The cross-cultural psychology literature has demonstrated on various occasions, that these dimensions account for most of the variance in global differences $[69,142]$. We demonstrated their effectiveness in a PT tool designed for individualist and collectivist audiences in New Zealand. But given that the dimensions of individualism and collectivism can be globally applied, the strategies are also applicable in many other cultural settings, to support the design of a range of tools and application domains. See $\S 4.3$ for a detailed description of the strategies.

\subsubsection{A prototype of a culturally-relevant persuasive technology}

Smoke? was designed and developed as a proof-of-concept culturally-relevant persuasive technology, based largely on our original culturally-relevant PT design strategies. The purpose of the Smoke? game was to increase players' antismoking attitudes, and to educate potential quitters about the quitting process. It is a simulation/adventure game where players guide the main character, $\mathrm{MC}$, in remaining quit over a six week period. It highlights activities that quitters can adopt to help manage nicotine cravings, provides players with cessation-related 
information, and illustrates scenarios that may arise during the quitting process that might be useful for smokers to plan for.

One version of Smoke? is designed to suit a NZ European player audience: the NZE version. The other version of Smoke? is designed to suit a Māori player audience: the NZM version. Focus groups with NZ European and Māori participants played an important role in the design of both game versions. The focus group feedback informed which of our PT strategies we used in the two versions, as well as how we featured them in their designs. Our development process spanned two iterations of development. To address issues in the original design concepts, and assess the usability of the game, we conducted a round of play testing on initial game versions. The evaluation findings led to design changes that we implemented in the final versions of the Smoke? game. See chapters 5, 6, and 7 for a detailed discussion of the PT development process.

\subsubsection{Evaluation of the culturally-relevant persuasive technology}

We performed separate quantitative and qualitative evaluations of Smoke? Both evaluations mostly indicated that when subjects played a game version that reflected their cultural orientation, they demonstrated greater changes in attitude than when they played a version that did not reflect their cultural orientation. The outcomes of these evaluations lend initial support for our research hypothesis, that persuasive technology is more effective when it reflects the culture of its intended target audience. See chapter 8 for a detailed discussion of the evaluations.

The quantitative evaluation involved the collection of the smoking-related perceptions of 141 New Zealanders before and after playing one of the two versions of Smoke? Individualist players reported a greater change in intention to quit when playing the NZE rather than the NZM version, while collectivist players reported a greater change in intention to quit when playing the NZM rather than the NZE version. Both the individualist and collectivist players reported a greater change in resistance to smoking when playing the NZM version. Unexpectedly, the individualist players reported a greater reduction in temptation to smoke when playing the NZM version, while the collectivist players reported a greater reduction when playing the NZE version, however we suggested ways to interpret these findings in the context of our overall hypothesis. Players of both versions of the game had increased anti-smoking beliefs after playing, as compared to their beliefs before playing.

The qualitative evaluation involved eight players performing the think aloud protocol, and interviewing after play. The NZ European players of the NZE ver- 
sion responded positively to, and made use of our game applications of the SELF MONITORING, TUNNELLING, and TAILORING strategies in the game. While claiming to welcome such features, the Māori players of the NZE version handled MC's social interactions differently from the NZ European players, perhaps indicating a lack of complete acceptance of the game. The Māori players of the NZM version responded positively towards, and engaged with our game applications of the HARMONY, MENTORING, and GROUP OPINION strategies, while the NZ European players found their presence puzzling, and at times, irritating.

\subsection{Delimitations and limitations of the study}

The generalisability of our results is limited in a number of ways.

Adopted perspective on culture The development phases of this work, including the analysis of the interviews with social marketing stakeholders, the examination of Fogg's strategies, the development of culturally-relevant PT strategies, and even the game design, draw on general concepts of individualism and collectivism (see §2.2.2.1), and the associated cross-cultural psychology literature.

The quantitative evaluation phase of this research, in contrast, made use of Schwartz's value theory [128]. We grouped players as individualist and collectivist according to their responses for the values survey. We then used this classification in our analysis of the effects of the game on players. It may be the case that using a different tool to determine individualistic or collectivistic orientations would have yielded different overall results.

The nature of our persuasive technology The form and topic of our persuasive technology determined which strategies we were able to implement during the design stage. We decided to use games as a platform, which limited us to using a subset of our design strategies, that is, strategies that could be harnessed in game design. In addition, we only incorporated strategies that supported the findings of focus groups about smoking cessation conducted with potential players. Consequently, from the entire set of culturally-relevant PT design strategies, there remain some that have not yet been used in tool designs.

Focus group members involved in the design phase of our persuasive technology As mentioned previously, one of the conditions for incorporating strategies into Smoke? was that they needed to be supported by findings of the focus groups we conducted with potential test players. We specifically recruited university-age 
NZ European and Māori participants for the focus groups, who may not have been representative of NZ European, or Māori culture in general.

Test players of our persuasive technology We conducted the evaluation phase of our study with mostly final year high-school students intending to attend university, and first year university students taking a certain psychology paper. Our survey included a question about self-identified culture, and an abbreviated version of Schwartz's values survey [128]. While we would have liked to control for variables such as age, gender, and socio-economic background, we focused our analysis on differentiation according to culture.

\subsection{Related work}

While few other studies have focused on culturally-relevant persuasive technology, our research findings are related to those of a number of other studies. These include studies from the fields of persuasive technology, $\mathrm{HCI}$, and consumer psychology. Here we discuss our findings in the context of those studies.

\subsubsection{PT research}

In $§ 2.1 .2 .1$, we overviewed the STOMP: Stop Smoking With Mobile Phones project, which is being conducted at the Clinical Trials Research Unit of the University of Auckland [28]. The findings of our study support the outcomes of the STOMP project, as both studies showed that cultural-relevance led to a greater degree of attitude change. Our findings also extend the STOMP project findings, as in our PT game, we not only referenced overt cues of Māori culture, we also referenced covert cues, by designing and implementing it to harness and reflect collectivist motivations. A notable difference between our study and the STOMP study was that the STOMP trial was longitudinal in nature, whereas in our evaluation, participants only played Smoke? once. Additionally, while we reported on players' intentional changes, the STOMP project researchers reported on behavioural changes that its users had established.

Other related PT research includes Grimes and Grinter's investigation of health technology for low income African American users (see §2.1.2.1) [57]. They discuss cultural-relevance as a guiding factor in health technology design, but focus on referencing concepts related to collectivism in technologies for African Americans. Grimes and Grinter's focus on cultural-relevance and games relates to our own research. Our study extends Grimes and Grinter's work, as we developed a game based on our own set of culturally-relevant PT strategies, and tested the 
game on players. Our findings support Grimes and Grinter's claim that culturalrelevance will yield effective technology.

In $§ 2.1 .2 .1$, we also discussed a virtual environment (VE) game, developed by the Virtual Reality Medical Center (VRMC). Like our own persuasive technology, it concerns presenting players with environments in which smoking triggers may occur. Our findings support those of the VRMC study. The test participants involved in our quantitative evaluation demonstrated a clear increase in anti-smoking beliefs after play, while the participants in the qualitative evaluation largely indicated that the game had led them to reflect on the quitting process. A clear difference between our research and that of the VRMC is that culture was not a factor of consideration in their study.

\subsubsection{Cultural aspects of HCI}

Our study findings relate to the research of Thomas Vöhringer-Kuhnt, who investigated the influence of culture on usability (see §2.3.3) [148]. Vöhringer-Kuhnt found that people's degree of individualism and collectivism impacted on their perceptions of usability, while other dimensions played a less appreciable role. In our research, we also focused on individualism and collectivism, but for the purpose of investigating differences in persuasiveness, rather than usability. Both our investigation and Vöhringer-Kuhnt's investigation emphasise the utility of the cultural dimensions of individualism and collectivism within an HCI context.

\subsubsection{Consumer psychology focused on individualism and col- lectivism}

In $§ 2.4 .2$, we discussed a number of cross-cultural consumer psychology studies investigating the effects of individualism and collectivism on consumer preferences. Our findings mostly support the results of the studies we reported, in that individualist and collectivist players responded in different ways to the NZE and NZM versions of Smoke? Unlike our study, however, the consumer psychology studies focused on print and television advertising, as opposed to persuasive technology. Additionally, most of these studies investigated the effectiveness of commercial appeals, as opposed to social marketing appeals.

\subsection{Future work}

This research represents one of a handful of studies linking culture and persuasive technology, and there remains much scope for further work. Here we discuss 
a few ways this research could be continued.

Using the Smoke? prototype By carrying out larger-scale quantitative testing on a wider range of users of the Smoke? game, it should be possible to begin investigating the effects of age, gender, and socio-economic background on player perceptions and attitude changes and analyse how these factors interact with the effects of culture on persuasive technology perception.

Additionally, it would be useful to revise the design and wording of the surveys, as some of the questions were less successful in eliciting analysable responses.

To obtain supplementary information relating to player experience, the game could be instrumented with timers and counters. These would provide objective information about which aspects of the game's interfaces the players spent time using.

It would also be useful to examine longitudinal effects of the game on players. This could be achieved by asking players to complete a follow-up attitude survey several days after the initial pre-survey, play, and post-survey session.

Finally, it would be interesting to see whether Smoke? could be incorporated as a component within a wider social marketing program, and how these components might function in collaboration to deliver a strong cohesive message, via multiple channels.

Developing a different game using a similar process The content of the Smoke? game was mostly related to smoking cessation. An interesting extension would be to develop another PT game, using the same development process and some of the same PT strategies as in Smoke?, but focusing on a different health-related issue, e.g. promotion of healthy nutrition and exercise. Using similar pre- and post-surveys as were developed for the quantitative evaluation of Smoke?, as well as qualitative evaluation methods, we would be in a position to start investigating wider generalisability of our research hypothesis. Furthermore, we could begin to establish the particular tasks that the individual design strategies lend themselves towards.

Developing a different persuasive technology using our design strategies As noted earlier, one of the limitations of our study is that we chose to develop a PT game. Consequently, we were limited in the strategies we were able to apply during the design process, and several of our strategies were not implemented in our game. A future direction would be to develop a completely different kind of persuasive technology implementing the remainder of the PT design strategies, 
to investigate their viability. A networked tool, in particular, would be interesting to develop, as there would be increased potential to implement the more sociallyoriented strategies (e.g. GROUP CUSTOMISATION and DIFFERENCE MONITORING). Again, this would give us further insight into the wider generalisability of our research hypothesis, and would allow us to explore the capacity of persuasive technology as social media.

\subsection{Summary}

A common way of explaining culture is to describe it as the lens through which we view the world. If indeed culture is so all-encompassing, then culture should be an important consideration factor in the overall process of development for persuasive technology.

In this thesis, we have investigated the relationship between culture and persuasive technology. We provided preliminary confirmation for the hypothesis that persuasive technology is more effective when it reflects the culture of its intended target audience, and made three solid contributions to the PT literature.

We developed an original and effective set of culturally-relevant PT design strategies, grounded in cross-cultural psychology literature on individualism and collectivism, for use by PT designers during the development process of a culturallyrelevant PT tool.

We demonstrated the use of several of the design strategies by developing two versions of a culturally-relevant PT game titled Smoke? on the topic of smoking cessation. One version has been designed for a NZ European player audience, and the other for a Māori player audience.

Finally, we evaluated both versions of the game on both a NZ European and Māori test population, using quantitative and qualitative measures. We showed that the NZE game version was more persuasive on the individualistic players, while the NZM game version was more persuasive on the collectivistic players. Overall, across both audiences and both game versions, people had increased anti-smoking beliefs after playing Smoke? compared to their beliefs before. 


\section{Appendix A}

\section{Case Study Information}

\section{Purpose of this Study}

I am undertaking a PhD investigating the relationship between culture and persuasive technology. Briefly, the major objectives of my research are:

1. Analysing existing persuasive technology strategies in the context of culture.

2. Developing persuasive technology strategies suited towards collectivist cultures, i.e. cultures in which self-identity is foremostly defined interdependently.

3. Performing case studies on persuasive websites, services, and their designers, to investigate implicitly used culturally-aware persuasion strategies.

4. Empirically evaluating proposed culturally-aware persuasive technology strategies.

This document contains information regarding the case studies objective listed above. Prior to any research involving human participants however, the University requires that ethics approval be obtained. I have already approached a member from this organisation regarding whether this organisation would be able to support this case study research, and received a positive response. The rest of this document therefore serves to further inform potential participants about their role and rights in this study.

\section{Nature of Participant Involvement}

Participants will be interviewed for up to 40 minutes, regarding persuasive website and service persuasion strategies and general campaign development. Participant interview responses will be audio taped, and will serve as research data. 
Opinions and information shared during the interview may be attributed to the participant in later research reports.

Participant interview material will enable me to develop a set of culturallyaware persuasive technology strategies. Hopefully, [organisation name] will be able to make use of such strategies in the future, as heuristics to guide culturallyconcerned projects. In turn, this may save time and money that may have otherwise been spent independently researching culture and persuasive technology issues.

Participants have the right to withdraw from the study without providing reasons or receiving any penalties until the [date of interview]. Notes and audio recordings for each interview will be destroyed 3 years after the conclusion of this research.

\section{Anonymity and confidentiality}

This research will not be conducted on a strictly anonymous basis, but access to the research data will be restricted to myself, my research supervisors, and an audio technician.

While I will also try my best to maintain information confidentiality by concealing participant names and using a pseuodonym for [organisation name], the public profile of [organisation name] and the small size of New Zealand make it difficult to keep organisation identity concealed. In turn, material shared during interview sessions could be unintentionally harmful for the reputation of [organisation name] and its participants.

To avoid inflicting unintentional damage to the reputation of [organisation name], after each interview, participants will be given the opportunity to review their interview transcripts for the purpose of making amendments. Any notes I make during observation sessions for my research will be used subject to approval by the observed group.

During interviews with participants, campaign sensitive information may also arise, that has not yet been released to the public. To avoid "leaking" such sensitive information, I will establish with [organisation name] a suitable date after which I may safely publish any reports including material gathered during this case study.

\section{Accessing Publications}

The material obtained through this case study will add to my general research, which will eventually culminate in a published $\mathrm{PhD}$ thesis, stored at the library 
of Victoria University of Wellington. This research may also result in other publications, such as conference posters, papers, and journal papers. Participants are welcome to access these publications if they wish to: the accompanying consent form contains an option regarding notification by email of any related publications.

\section{Contact Details}

If participants have any questions regarding their involvement in this case study, or issues regarding the case study in general, please do not hesitate to contact me:

Rilla Khaled Principal researcher

School of Mathematics, Statistics, and Computer Science

rkhaled@mcs.vuw.ac.nz

Professor James Noble Primary Supervisor

School of Mathematics, Statistics, and Computer Science

kjx@mcs.vuw.ac.nz 


\section{Appendix B}

\section{Case Study Consent Form}

I have been provided with adequate information relating to the nature and objectives of this research project, and have been given the opportunity to ask questions about the research.

I agree to be interviewed and/or observed by Rilla Khaled as part of a case study forming a portion of her PhD research. I understand that the information I provide will be audio taped and used only for this research project and that any further use will require my written consent. Additionally, I am aware that this research may be published in the form of conference posters, papers, and journal papers, as well as in the form of a PhD thesis.

I understand that the nature of [insert organisation name], and the matters that will be discussed during interviews, will make it difficult to maintain information confidentiality. I therefore consent to information, including opinions, recollections, facts, and statistics being attributed to me, subject to the concealment of both the identity of [insert organisation name] and my own identity. In the case of discussion arising concerning sensitive campaign information, I understand that the researcher will not publish any results regarding this sensitive information until a time at which [insert organisation name] is comfortable with its public release.

I understand that I have the right to withdraw from this study until the [insert date of interview] without providing reasons or suffering penalties, and may review any transcripts resulting from interview/observation sessions. I am also aware that all information obtained as a consequence of my participation will be destroyed within 3 years.

Do you have consent from your employer to partake in this case study?

Do you want to be notified of any publications relating to this research? 
Email address or phone number at which you may be contacted regarding publications:

Name:

Signed:

Date:

If participants have any questions regarding their involvement in my research, or issues regarding the research in general, please do not hesitate to contact me:

Rilla Khaled Principal researcher

School of Mathematics, Statistics, and Computer Science rkhaled@mcs.vuw.ac.nz

James Noble Primary Supervisor

School of Mathematics, Statistics, and Computer Science kjx@mcs.vuw.ac.nz 


\section{Appendix C}

\section{Case Study Interview Outline}

\section{Purpose}

The purpose of this interview is to investigate how designers of persuasive websites and other persuasive services in New Zealand are currently approaching the task of communicating persuasion appeals, and their reasons for using various persuasive strategies.

\section{Discussion topics}

- Persuasion tactics currently used within the organisations website and/or other media (10 minutes):

- What tactics are used e.g. family based appeals, personal responsibility appeals, etc.?

- How are these strategies decided upon?

- What is the role of academic research in the strategy designing process?

- Target audiences (5 minutes):

- What level of grouping is used to divide the audience, e.g. age groups, cultural groups, religious beliefs?

- How are representatives of these groups accessed?

- Why are these particular accessing mechanisms used?

- Target audience input (5 minutes):

- How are opinions gathered from target audience representatives i.e. focus groups, etc.? 
- In the general case, how much does target audience input affect the direction any particular campaign takes?

- Campaign development (10 minutes):

- What is the typical development lifecycle for a campaign regarding target audience input and strategy development, from start to finish?

- What steps are taken to ensure that the tactics aimed at a particular target audience will be effective upon this audience, i.e. what testing mechanisms are used?

- Campaign lifecycles (10 minutes):

- Is the campaign modified over time in accordance with audience feedback?

- What is the time duration for a typical campaign? 


\section{Appendix D}

\section{Focus Group Information}

\section{Purpose of this Study}

I am currently undertaking a PhD investigating the relationship between culture and persuasive technology. Persuasive technology is any interactive product i.e. a website, that is designed to change attitudes or behaviours by making desired outcomes easier to achieve. Consumer behaviour research shows us that for persuasion to be successful, it needs to make use of appeals that will be interesting and relevant to its target audience.

One of my overall project objectives therefore, is to develop a set of culturallyaware persuasive technology design considerations, e.g. guidelines that designers of health and well-being websites such as the Ministry of Health-run "No Rubba No Hubba Hubba" site, and the Retirement Commission-run "Get Sorted" site, could incorporate into their websites. To develop these guidelines, I would like to investigate how people of different ethnicities view and use persuasive services and technologies.

Prior to any research involving human participants however, the University requires that ethics approval be obtained. The rest of this document therefore serves to further inform potential participants about their role and rights in this study.

\section{Nature of Participant Involvement}

I would like to conduct a series of focus groups with people who identify as being [insert group name], to investigate how they view and use persuasive services and technologies. Another aim is to gain initial insight into how people from this ethnic group might react to particular persuasive technology strategies. This focus group material will eventually be used to guide the development of interactive websites addressing health and well-being needs. In particular, this 
research can be applied to websites focusing on raising awareness and modifying behaviours related to smoking, sexual health, cancer screening, exercise and diet, and gambling. It could also be used to design culturally-relevant educational tools, develop communities of learning, develop mentoring networks, and provide technological means for existing community support systems.

The focus group session will last approximately 6090 minutes. Participant discussion will be audio taped, and will serve as research data. While the opinions and information shared during the focus group may be discussed in later reports concerning this case study, the identities of individuals will always remain concealed. Notes and audio recordings for each focus group will be destroyed 3 years after the conclusion of this research.

Participants have the right to withdraw from the study without providing reasons until the [insert date]. Withdrawal will result in the exclusion of their discussion input from my research, however I may not be able to destroy their audio recording, as it will be part of the entire group discussion audio recording.

\section{Anonymity and confidentiality}

This research will not be conducted on a strictly anonymous basis, as I will be present, along with the rest of the focus group participants. It will however be confidential, as access to the research data will be restricted to myself, my research supervisors, and an audio technician. Furthermore, what a participants hears and shares during each session should be treated as confidential. Although opinions and information shared during the session may be discussed in later publications, identities will always be concealed.

\section{Accessing Publications}

The material obtained through this focus group will add to my general research, which will eventually be stored at the library of Victoria University of Wellington. This research may also result in other publications, such as conference posters, papers, and journal papers. Participants are welcome to access these publications if they wish to: the accompanying consent form contains an option regarding notification by email of any related publications.

\section{Contact Details}

If participants have any questions regarding their involvement in this case study, or issues regarding the case study in general, please do not hesitate to contact me: 
Rilla Khaled Principal researcher

School of Mathematics, Statistics, and Computer Science rkhaled@mcs.vuw.ac.nz

Professor James Noble Primary Supervisor

School of Mathematics, Statistics, and Computer Science kjx@mcs.vuw.ac.nz 


\section{Appendix E}

\section{Focus Group Consent Form}

I have been provided with adequate information relating to the nature and objectives of this research project, and have been given the opportunity to ask questions about the research.

I agree to participate in a focus group as part of Rilla Khaled's PhD research. I understand that the information I provide will be recorded and used only for this research project and that any further use will require my written consent. Additionally, I am aware that this research may be published in the form of conference posters, papers, and journal papers, as well as in the form of a $\mathrm{PhD}$ thesis.

I consent to information, including opinions, recollections, facts, and statistics being attributed to me, subject to the concealment of my identity. Furthermore, I agree to treat opinions and information heard and shared during the focus group session as confidential.

I understand that I have the right to withdraw from this study until the 20th of February without providing reasons. I am also aware that any data obtained as a consequence of my participation (i.e. audio tapes, transcripts, notes) will be destroyed either within 3 years, or if I withdraw from the study, immediately upon my withdrawal.

I understand that I have the right to withdraw from this study until the [insert date of interview] without providing reasons or suffering penalties, and may review any transcripts resulting from interview/observation sessions. I am also aware that all information obtained as a consequence of my participation will be destroyed within 3 years.

Would you like to be sent the resulting smoking cessation game?

Do you want to be notified of any publications relating to this research? 
Email address or phone number at which you may be contacted regarding publications:

Name:

Signed:

Date:

If participants have any questions regarding their involvement in my research, or issues regarding the research in general, please do not hesitate to contact me:

Rilla Khaled Principal researcher

School of Mathematics, Statistics, and Computer Science rkhaled@mcs.vuw.ac.nz

James Noble Primary Supervisor

School of Mathematics, Statistics, and Computer Science kjx@mcs.vuw.ac.nz 


\section{Appendix F}

\section{Focus Group Agenda}

\section{Purpose}

The main goal of this focus group is to investigate how people who identify as [insert group name] view and use persuasive services and technologies. Another aim is to gain initial insight into how people who identify as [insert group name] might react to particular persuasive technology strategies, and obtain design ideas about culturally-aware persuasive technologies.

\section{Discussion topics}

- PART ONE: General Discussion:

- Being a target audience:

* Campaigns where you have been a target audience

* Types of appeals used

* Success of appeals

* Feedback mechanisms

- Convincing persuasion techniques and strategies:

* Effective: self-focused, other-focused, guilt-driven, shame-driven, etc.

* Ineffective: self-focused, other-focused, guilt-driven, shame-driven, etc.

* Situational relevance of appeals

- PART TWO: Smoking:

- Attitudes towards smoking

- Smoking in the media 
- Suggestions for deterrence strategies

- PART THREE: New persuasive technology strategies

- Effective hypothetical strategies

- Ineffective hypothetical strategies 


\section{Appendix G}

\section{Game Tester Information}

\section{Research Overview}

I am doing a PhD investigating the relationship between culture and persuasive technology. Persuasive technology is any interactive product i.e. a website, that is designed to change attitudes or behaviours by making desired outcomes easier to achieve. I have developed two versions of a computer game called Smoke? about smoking cessation. One version of Smoke? is designed to appeal to Pakeha audiences, while the other is designed to appeal to Māori and PI audiences. I am interested in investigating whether Pakeha game players find the Pakeha version of the game more persuasive than the Māori/PI version, and equally, whether the Māori and PI participants find the Māori/PI version of the game more persuasive.

This document serves to inform you as a potential participant about your role and rights in this study.

\section{Nature of Involvement}

I am interested in finding out about your experiences while playing Smoke?. I would like to survey you about your attitudes towards particular aspects of smoking cessation, before game play. Following this initial survey session, I would like you to play either the Pakeha or the Māori/PI version of the game for approximately 40 minutes. Afterwards, I would again like to survey you. Carrying out two survey rounds will allow me to compare how much attitude shift the game has induced, if any, and whether one game causes more attitude shift on one audience than another.

The survey and game play session itself will last approximately 60 minutes. While the attitudes, opinions, and information shared during the session may be discussed in later reports and publications, identity information will always remain concealed. Surveys and notes related to game testing will be destroyed 3 
years after the conclusion of this research.

You have the right to withdraw from the study without providing reasons until the [insert date]. Withdrawal will result in the exclusion of your survey data from my research.

\section{Anonymity and confidentiality}

This research will not be conducted on a strictly anonymous basis, as I may be present. It will however be confidential, as access to the research data will be restricted to myself, and my research supervisors. Although opinions and information shared during the session may be discussed in later publications, identities will always be concealed.

\section{Accessing Publications}

The material obtained through game testing will add to my general research, which will eventually be stored at the library of Victoria University of Wellington. This research may also result in other publications, such as conference posters, papers, and journal papers. You are welcome to access these publications if you so wish: the accompanying consent form contains an option regarding notification by email of any related publications.

\section{Contact Details}

If you have any questions regarding your involvement in my research, or issues regarding the research in general, please do not hesitate to contact me:

Rilla Khaled Principal researcher

School of Mathematics, Statistics, and Computer Science

rkhaled@mcs.vuw.ac.nz

Professor James Noble Primary Supervisor

School of Mathematics, Statistics, and Computer Science

kjx@mcs.vuw.ac.nz 


\section{Appendix $\mathbf{H}$}

\section{Game Tester Consent Form}

I have been provided with adequate information relating to the nature and objectives of this research project, and have been given the opportunity to ask questions about the research.

I agree to participate as a test participant for the game Smoke? as part of Rilla Khaled's PhD research. I understand that the information I provide by way of completing surveys will be used only for this research project and that any further use will require my written consent. Additionally, I am aware that this research may be published in the form of conference posters, papers, and journal papers, as well as in the form of a PhD thesis.

I consent to information, including attitudes, opinions, recollections, and facts, being attributed to me, subject to the concealment of my identity.

I understand that I have the right to withdraw from this study until the [insert date], without providing reasons. I am also aware that any data obtained as a consequence of my participation (i.e. survey data, audio tapes, transcripts, notes) will be destroyed either within 3 years, or if I withdraw from the study, immediately upon my withdrawal.

Do you want to be notified of any publications relating to this research?

Email address or phone number at which you may be contacted regarding publications:

Name:

Signed:

Date: 
If participants have any questions regarding their involvement in my research, or issues regarding the research in general, please do not hesitate to contact me:

Rilla Khaled Principal researcher

School of Mathematics, Statistics, and Computer Science rkhaled@mcs.vuw.ac.nz

Professor James Noble Primary Supervisor

School of Mathematics, Statistics, and Computer Science kjx@mcs.vuw.ac.nz 


\section{Appendix I}

\section{Smoke survey}

The following pages include the survey given to all participants of the quantitative evaluation, as described in Chapter 8. 


\section{PRE-TEST SURVEY}

1) Please rate how important each value is for you as a guiding principle in your life. In the space before each value, write the number $(0,1,2,3,4,5,6,7)$ that indicates the importance of that value for you, personally. Try to distinguish as much as possible between the values by using all the numbers. You will, of course, need to use numbers more than once.

AS A GUIDING PRINCIPLE IN MY LIFE, this value is:

$\begin{array}{cccccccc}\text { Not } & \text { Almost not } & \text { Slightly } & \text { Quite } & \text { Important } & \text { Very } & \text { Extremely } & \text { Of supreme } \\ \text { important } & \text { important } & \text { important } & \text { important } & \text { important } & \text { important } & \text { importance } \\ 0 & 1 & 2 & 3 & 4 & 5 & 6 & 7\end{array}$

\section{TRY TO USE THE RATING ‘OF SUPREME IMPORTANCE’ NOT MORE THEN TWICE}

Before you begin, read the values in List I, choose the one that is most important to you and rate its importance. Next, choose the value least important to you and rate it 0 or 1, according to its importance. Then rate the rest of the values in List I. FREEDOM (freedom of action and thought)

2 SENSE OF BELONGING (feeling that others care about me) SELF RESPECT (belief in one's own worth) CREATIVITY (uniqueness, imagination) RESPECT FOR TRADITION (preservation of time-honored customs) SELF-DISCIPLINE (self-restraint, resistance to temptation) PRIVACY (the right to have a private sphere) SOCIAL RECOGNITION (respect, approval by others) INDEPENDENT (self-reliant, self-sufficient) BROADMINDED (tolerant of different ideas and beliefs) HONORING OF PARENTS AND ELDERS (showing respect) CHOOSING OWN GOALS (selecting own purposes) CAPABLE (competent, effective, efficient) ACCEPTING MY PORTION IN LIFE (submitting to life's circumstances) PRESERVING MY PUBLIC IMAGE (protecting my "face") HELPFUL (working for the welfare of others) 


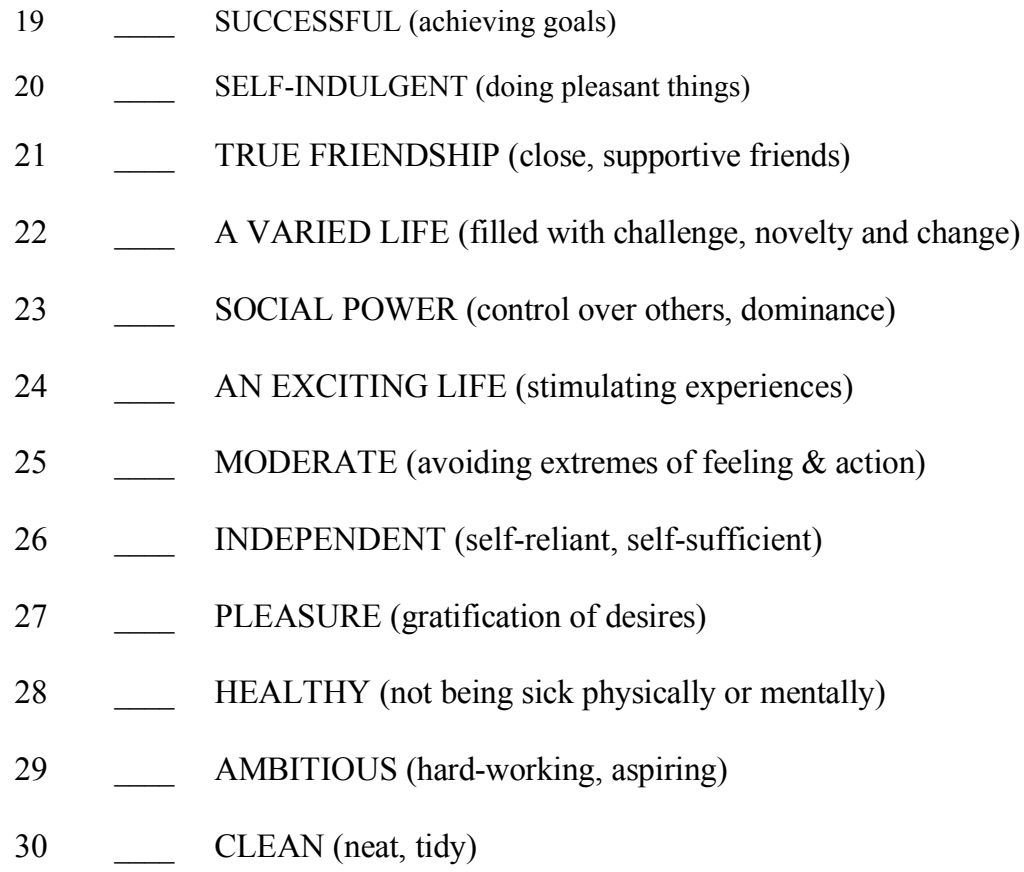

\section{Attitudes towards smoking.}

2) In the following table you will see a number of statements. Please indicate how much you disagree or agree with each statement. Please remember there are no right or wrong answers.

\begin{tabular}{|c|c|c|c|c|c|}
\hline & $\begin{array}{l}\text { Strongly } \\
\text { disagree }\end{array}$ & $\begin{array}{c}\text { Disagr } \\
\text { ee }\end{array}$ & $\begin{array}{l}\text { Neither agree } \\
\text { nor disagree }\end{array}$ & Agree & $\begin{array}{c}\begin{array}{c}\text { Strongly } \\
\text { agree }\end{array} \\
\text { on }\end{array}$ \\
\hline When you are stressed, smoking helps to relax & 1 & 2 & 3 & 4 & 5 \\
\hline If you smoke, you spend a lot of money on cigarettes & 1 & 2 & 3 & 4 & 5 \\
\hline Smokers are more relaxed than non-smokers & 1 & 2 & 3 & 4 & 5 \\
\hline $\begin{array}{l}\text { Smoking has negative consequences for other people } \\
\text { who do not smoke }\end{array}$ & 1 & 2 & 3 & 4 & 5 \\
\hline Smoking makes you look more mature & 1 & 2 & 3 & 4 & 5 \\
\hline Smokers get out of breath more easily & 1 & 2 & 3 & 4 & 5 \\
\hline Smoking is attractive & 1 & 2 & 3 & 4 & 5 \\
\hline Smoking is a good way to reduce your weight & 1 & 2 & 3 & 4 & 5 \\
\hline Smokers are more attractive & 1 & 2 & 3 & 4 & 5 \\
\hline Smokers are bad role-models & 1 & 2 & 3 & 4 & 5 \\
\hline Smokers have more friends & 1 & 2 & 3 & 4 & 5 \\
\hline Smokers are more social than non-smokers & 1 & 2 & 3 & 4 & 5 \\
\hline Smoking causes bad breath & 1 & 2 & 3 & 4 & 5 \\
\hline Non-smokers save more money & 1 & 2 & 3 & 4 & 5 \\
\hline Smokers have a higher risk of getting a serious illness & 1 & 2 & 3 & 4 & 5 \\
\hline Smokers are more fashionable & 1 & 2 & 3 & 4 & 5 \\
\hline Smokers smell bad & 1 & 2 & 3 & 4 & 5 \\
\hline
\end{tabular}




\begin{tabular}{llllll}
\hline $\begin{array}{l}\text { If you smoke, you damage the health of your friends } \\
\text { and family }\end{array}$ & 1 & 2 & 3 & 4 & 5 \\
\hline Smoking makes teeth go yellow & 1 & 2 & 3 & 4 & 5 \\
\hline Smoking has a bad effect on other people & 1 & 2 & 3 & 4 & 5 \\
\hline Smoking also makes other people sick & 1 & 2 & 3 & 4 & 5 \\
\hline
\end{tabular}

3) Next, you will see some statements about what your friends might think. Please indicate to what extent these statements are true for your current group of friends.

\begin{tabular}{lccccc}
\hline & $\begin{array}{l}\text { Strongly } \\
\text { disagree }\end{array}$ & $\begin{array}{c}\text { Disagr } \\
\text { ee }\end{array}$ & $\begin{array}{l}\text { Neither agree } \\
\text { nor disagree }\end{array}$ & $\begin{array}{c}\text { Agree } \\
\text { Strongly } \\
\text { agree }\end{array}$ \\
\hline My friends think that smoking is irresponsible & 1 & 2 & 3 & 4 & 5 \\
\hline My friends think that smoking is a bad habit & 1 & 2 & 3 & 4 & 5 \\
\hline My friends think that smokers are attractive & 1 & 2 & 3 & 4 & 5 \\
\hline My friends think that smoking makes people sick & 1 & 2 & 3 & 4 & 5 \\
\hline My friends think that smoking is cool & 1 & 2 & 3 & 4 & 5 \\
\hline
\end{tabular}

4) Next, you will find a series of concepts written in bold. Rate the concept according to how closely you feel it is related to the characteristic at each end.

\begin{tabular}{lllllllll} 
& \multicolumn{7}{c}{ Smoking } \\
\hline Bad & 1 & 2 & 3 & 4 & 5 & 6 & 7 & Good \\
\hline Safe & 1 & 2 & 3 & 4 & 5 & 6 & 7 & Dangerous \\
\hline Beautiful & 1 & 2 & 3 & 4 & 5 & 6 & 7 & Ugly \\
\hline Strong & 1 & 2 & 3 & 4 & 5 & 6 & 7 & Weak \\
\hline Healthy & 1 & 2 & 3 & 4 & 5 & 6 & 7 & Sick \\
\hline Tasty & 1 & 2 & 3 & 4 & 5 & 6 & 7 & Distasteful \\
\hline
\end{tabular}

\begin{tabular}{lllllllll}
\multicolumn{8}{c}{ Smokers } \\
\hline Bad & 1 & 2 & 3 & 4 & 5 & 6 & 7 & Good \\
\hline Safe & 1 & 2 & 3 & 4 & 5 & 6 & 7 & Dangerous \\
\hline Beautiful & 1 & 2 & 3 & 4 & 5 & 6 & 7 & Ugly \\
\hline Strong & 1 & 2 & 3 & 4 & 5 & 6 & 7 & Weak \\
\hline Healthy & 1 & 2 & 3 & 4 & 5 & 6 & 7 & Sick \\
\hline Calm & 1 & 2 & 3 & 4 & 5 & 6 & 7 & Agitated \\
\hline Passive & 1 & 2 & 3 & 4 & 5 & 6 & 7 & Active \\
\hline
\end{tabular}

5) In the following you will see a number of statements. Please indicate how much you disagree or agree with each statement. Please remember there are no right or wrong answers.

\begin{tabular}{lccccc}
\hline Smoking can make people ill, but & $\begin{array}{c}\text { Strongly } \\
\text { disagree }\end{array}$ & Disagree & $\begin{array}{c}\text { Neither } \\
\text { agree } \\
\text { nor } \\
\text { disagree }\end{array}$ & $\begin{array}{c}\text { Agree } \\
\text { agree }\end{array}$ \\
\hline Pollution is just as unhealthy & 1 & 2 & 3 & 4 & 5 \\
\hline There are lots of risks in life & 1 & 2 & 3 & 4 & 5 \\
\hline Everything is unhealthy & 1 & 2 & 3 & 4 & 5 \\
\hline
\end{tabular}




\begin{tabular}{llllll}
\hline You have to die of something & 1 & 2 & 3 & 4 & 5 \\
\hline Everyone acts unhealthy sometimes & 1 & 2 & 3 & 4 & 5 \\
\hline Not everyone gets sick smoking & 1 & 2 & 3 & 4 & 5 \\
\hline I know heavy smokers that lived long & 1 & 2 & 3 & 4 & 5 \\
\hline Rather a short and good life than a long and boring life & 1 & 2 & 3 & 4 & 5 \\
\hline Health is not the only thing in life & 1 & 2 & 3 & 4 & 5 \\
\hline
\end{tabular}

6) Now, I would like to ask you some questions about yourself. You will never be personally identified in this research project or in any presentation or publication. The information you provide will be coded by number, and used for statistical purposes only.

i. How many of you best four female friends do smoke?

\begin{tabular}{|l|l|l|l|l|} 
None & One & Two & Three & All \\
\hline
\end{tabular}

ii. How many of your best four male friends do smoke?

\begin{tabular}{|l|l|l|l|l|} 
None & One & Two & Three & All \\
\hline
\end{tabular}

iii. How many people in your family do smoke? (please list)

iv. How many people in the house you are currently living in are smoking regularly? (please list the number of people currently smoking).

v. Please indicate your ethnic identity (e.g., New Zealand European, Maori, Samoan):

7) If you are currently a smoker or if you have smoked before, please answer this section. If you have never smoked in your life, please go to question 8.

i. When did you smoke your last cigarette?

ii. What label would best describe your smoking behaviour?

\begin{tabular}{|c|c|c|c|}
\hline Ex-smoker & Light smoker & Moderate smoker & Heavy smoker \\
\hline
\end{tabular}

iii. How often do you smoke?

\begin{tabular}{|c|c|c|c|c|c|}
\hline Once a month & $\begin{array}{c}\text { Once a } \\
\text { fortnight }\end{array}$ & Once a week & Twice a week & $\begin{array}{c}\text { Three times a } \\
\text { week }\end{array}$ & $\begin{array}{c}\text { Every day or } \\
\text { nearly every } \\
\text { day }\end{array}$ \\
\hline
\end{tabular}

iv. If you don't smoke currently, how many cigarettes have you smoked in the past? 
v. How long did you smoke?

\begin{tabular}{|c|c|c|c|c|c|c|}
\hline $\begin{array}{c}\text { Tried only } \\
\text { once }\end{array}$ & One week & Once month & $\begin{array}{c}\text { A few } \\
\text { months }\end{array}$ & Half a year & One year & $\begin{array}{c}\text { A few } \\
\text { years }\end{array}$ \\
\hline
\end{tabular}

vi. How would you rate your chances of quitting smoking?

\begin{tabular}{lllllll}
\hline & $\begin{array}{l}\text { Very } \\
\text { Low }\end{array}$ & & & & $\begin{array}{c}\text { Very } \\
\text { High }\end{array}$ \\
\hline In the next week & 1 & 2 & 3 & 4 & 5 & 7 \\
\hline In the next month & 1 & 2 & 3 & 4 & 5 & 7 \\
\hline In the next three months & 1 & 2 & 3 & 4 & 5 & 7 \\
\hline In the next six months & 1 & 2 & 3 & 4 & 5 & 7 \\
\hline Sometime in the next year & 1 & 2 & 3 & 4 & 5 & 7 \\
\hline
\end{tabular}

8)

If you are a non-smoker, please answer the following questions.

\begin{tabular}{lccccc}
\hline & $\begin{array}{c}\text { Strongly } \\
\text { disagree }\end{array}$ & $\begin{array}{c}\text { Disag } \\
\text { ree }\end{array}$ & $\begin{array}{c}\text { Neither } \\
\text { agree nor } \\
\text { disagree }\end{array}$ & $\begin{array}{c}\text { Agree } \\
\text { agree }\end{array}$ & $\begin{array}{c}\text { Strongly } \\
\text { agree }\end{array}$ \\
\hline If a friend would offer me a cigarette, I would try it & 1 & 2 & 3 & 4 & 5 \\
\hline I am actively avoiding situations where people smoke & 1 & 2 & 3 & 4 & 5 \\
\hline When pressured by friends, I would try smoking & 1 & 2 & 3 & 4 & 5 \\
\hline If cigarettes were cheaper, I would start smoking & 1 & 2 & 3 & 4 & 5 \\
\hline $\begin{array}{l}\text { If in a group, I would smoke so that I am not an } \\
\text { outsider }\end{array}$ & 1 & 2 & 3 & 4 & 5 \\
\hline I feel like an outsider when my friends smoke & 1 & 2 & 3 & 4 & 5 \\
\hline $\begin{array}{l}\text { Even when my friends try and persuade me, I would } \\
\text { resist smoking }\end{array}$ & 1 & 2 & 3 & 4 & 5 \\
\hline I am too shy to try smoking, but I would like to. & 1 & 2 & 3 & 4 & 5 \\
\hline I would like to smoke, but I can't afford it. & 1 & 2 & 3 & 4 & 5 \\
\hline I will talk to my friends about quitting smoking & 1 & 2 & 3 & 4 & 5 \\
\hline Smoking bothers me & 1 & 2 & 3 & 4 & 5 \\
\hline $\begin{array}{l}\text { I will talk to my smoking friends to persuade them to } \\
\text { stop }\end{array}$ & 1 & 2 & 3 & 4 & 5 \\
\hline I am tempted to try a cigarette & 1 & 2 & 3 & 4 & 5 \\
\hline I would accept a cigarette when friends offer me one & 1 & 2 & 3 & 4 & 5 \\
\hline
\end{tabular}




\section{POST-TEST SURVEY}

\section{Attitudes towards smoking.}

9) In the following table you will see a number of statements. Please indicate how much you disagree or agree with each statement. Please remember there are no right or wrong answers.

\begin{tabular}{llllll}
\hline & $\begin{array}{l}\text { Strongly } \\
\text { disagree }\end{array}$ & $\begin{array}{c}\text { Disagr } \\
\text { ee }\end{array}$ & $\begin{array}{l}\text { Neither agree } \\
\text { nor disagree }\end{array}$ & $\begin{array}{c}\text { Agree } \\
\text { agree }\end{array}$ \\
\hline When you are stressed, smoking helps to relax & 1 & 2 & 3 & 4 & 5 \\
\hline If you smoke, you spend a lot of money on cigarettes & 1 & 2 & 3 & 4 & 5 \\
\hline Smokers are more relaxed than non-smokers & 1 & 2 & 3 & 4 & 5 \\
\hline $\begin{array}{l}\text { Smoking has negative consequences for other people } \\
\text { who do not smoke }\end{array}$ & 1 & 2 & 3 & 4 & 5 \\
\hline Smoking makes you look more mature & 1 & 2 & 3 & 4 & 5 \\
\hline Smokers get out of breath more easily & 1 & 2 & 3 & 4 & 5 \\
\hline Smoking is attractive & 1 & 2 & 3 & 4 & 5 \\
\hline Smoking is a good way to reduce your weight & 1 & 2 & 3 & 4 & 5 \\
\hline Smokers are more attractive & 1 & 2 & 3 & 4 & 5 \\
\hline Smokers are bad role-models & 1 & 2 & 3 & 4 & 5 \\
\hline Smokers have more friends & 1 & 2 & 3 & 4 & 5 \\
\hline Smokers are more social than non-smokers & 1 & 2 & 3 & 4 & 5 \\
\hline Smoking causes bad breath & 1 & 2 & 3 & 4 & 5 \\
\hline Non-smokers save more money & 1 & 2 & 3 & 4 & 5 \\
\hline Smokers have a higher risk of getting a serious illness & 1 & 2 & 3 & 4 & 5 \\
\hline Smokers are more fashionable & 1 & 2 & 3 & 4 & 5 \\
\hline Smokers smell bad & 1 & 2 & 3 & 4 & 5 \\
\hline If you smoke, you damage the health of your friends & 1 & 2 & 3 & 4 & 5 \\
and family & & & & 4 & 5 \\
\hline Smoking makes teeth go yellow & 1 & 2 & 3 & 4 & 5 \\
\hline Smoking has a bad effect on other people & 1 & 2 & 3 & 4 & 5 \\
\hline Smoking also makes other people sick & 1 & 2 & 3 & & 5 \\
\hline
\end{tabular}

10) Next, you will find a series of concepts written in bold. Rate the concept according to how closely you feel it is related to the characteristic at each end.

\begin{tabular}{lllllllll} 
& \multicolumn{7}{c}{ Smoking } \\
\hline Bad & 1 & 2 & 3 & 4 & 5 & 6 & 7 & Good \\
\hline Safe & 1 & 2 & 3 & 4 & 5 & 6 & 7 & Dangerous \\
\hline Beautiful & 1 & 2 & 3 & 4 & 5 & 6 & 7 & Ugly \\
\hline Strong & 1 & 2 & 3 & 4 & 5 & 6 & 7 & Weak \\
\hline Healthy & 1 & 2 & 3 & 4 & 5 & 6 & 7 & Sick \\
\hline Tasty & 1 & 2 & 3 & 4 & 5 & 6 & 7 & Distasteful \\
\hline
\end{tabular}


Smokers

\begin{tabular}{lllllllll}
\hline Bad & 1 & 2 & 3 & 4 & 5 & 6 & 7 & Good \\
\hline Safe & 1 & 2 & 3 & 4 & 5 & 6 & 7 & Dangerous \\
\hline Beautiful & 1 & 2 & 3 & 4 & 5 & 6 & 7 & Ugly \\
\hline Strong & 1 & 2 & 3 & 4 & 5 & 6 & 7 & Weak \\
\hline Healthy & 1 & 2 & 3 & 4 & 5 & 6 & 7 & Sick \\
\hline Calm & 1 & 2 & 3 & 4 & 5 & 6 & 7 & Agitated \\
\hline Passive & 1 & 2 & 3 & 4 & 5 & 6 & 7 & Active \\
\hline
\end{tabular}

11) In the following you will see a number of statements. Please indicate how much you disagree or agree with each statement. Please remember there are no right or wrong answers.

\begin{tabular}{lccccc}
\hline Smoking can make people ill, but & $\begin{array}{c}\text { Strongly } \\
\text { disagree }\end{array}$ & Disagree & $\begin{array}{c}\text { Neither } \\
\text { agree } \\
\text { nor } \\
\text { disagree }\end{array}$ & $\begin{array}{c}\text { Agree } \\
\text { Strongly } \\
\text { agree }\end{array}$ \\
\hline Pollution is just as unhealthy & 1 & 2 & 3 & 4 & 5 \\
\hline There are lots of risks in life & 1 & 2 & 3 & 4 & 5 \\
\hline Everything is unhealthy & 1 & 2 & 3 & 4 & 5 \\
\hline You have to die of something & 1 & 2 & 3 & 4 & 5 \\
\hline Everyone acts unhealthy sometimes & 1 & 2 & 3 & 4 & 5 \\
\hline Not everyone gets sick smoking & 1 & 2 & 3 & 4 & 5 \\
\hline I know heavy smokers that lived long & 1 & 2 & 3 & 4 & 5 \\
\hline Rather a short and good life than a long and boring life & 1 & 2 & 3 & 4 & 5 \\
\hline Health is not the only thing in life & 1 & 2 & 3 & 4 & 5 \\
\hline
\end{tabular}

12) If you are a smoker, how would you rate your chances of quitting smoking?

\begin{tabular}{|c|c|c|c|c|c|c|}
\hline & $\begin{array}{l}\text { Very } \\
\text { Low }\end{array}$ & & & & & $\begin{array}{l}\text { Very } \\
\text { High }\end{array}$ \\
\hline In the next week & 1 & 2 & 3 & 4 & 5 & 7 \\
\hline In the next month & 1 & 2 & 3 & 4 & 5 & 7 \\
\hline In the next three months & 1 & 2 & 3 & 4 & 5 & 7 \\
\hline In the next six months & 1 & 2 & 3 & 4 & 5 & 7 \\
\hline Sometime in the next year & 1 & 2 & 3 & 4 & 5 & 7 \\
\hline
\end{tabular}




\begin{tabular}{|c|c|c|c|c|c|}
\hline & $\begin{array}{l}\text { Strongly } \\
\text { disagree }\end{array}$ & $\begin{array}{c}\text { Disag } \\
\text { ree }\end{array}$ & $\begin{array}{c}\text { Neither } \\
\text { agree nor } \\
\text { disagree }\end{array}$ & Agree & $\begin{array}{c}\text { Strongly } \\
\text { agree }\end{array}$ \\
\hline If a friend would offer me a cigarette, I would try it & 1 & 2 & 3 & 4 & 5 \\
\hline I am actively avoiding situations where people smoke & 1 & 2 & 3 & 4 & 5 \\
\hline When pressured by friends, I would try smoking & 1 & 2 & 3 & 4 & 5 \\
\hline If cigarettes were cheaper, I would start smoking & 1 & 2 & 3 & 4 & 5 \\
\hline $\begin{array}{l}\text { If in a group, I would smoke so that I am not an } \\
\text { outsider }\end{array}$ & 1 & 2 & 3 & 4 & 5 \\
\hline I feel like an outsider when my friends smoke & 1 & 2 & 3 & 4 & 5 \\
\hline $\begin{array}{l}\text { Even when my friends try and persuade me, I would } \\
\text { resist smoking }\end{array}$ & 1 & 2 & 3 & 4 & 5 \\
\hline I am too shy to try smoking, but I would like to. & 1 & 2 & 3 & 4 & 5 \\
\hline I would like to smoke, but I can't afford it. & 1 & 2 & 3 & 4 & 5 \\
\hline I will talk to my friends about quitting smoking & 1 & 2 & 3 & 4 & 5 \\
\hline Smoking bothers me & 1 & 2 & 3 & 4 & 5 \\
\hline $\begin{array}{l}\text { I will talk to my smoking friends to persuade them to } \\
\text { stop }\end{array}$ & 1 & 2 & 3 & 4 & 5 \\
\hline I am tempted to try a cigarette & 1 & 2 & 3 & 4 & 5 \\
\hline I would accept a cigarette when friends offer me one & 1 & 2 & 3 & 4 & 5 \\
\hline
\end{tabular}

14) We would like to know what you thought of the game 'Smoke?'. Please answer how much agree with each of the statements.

\begin{tabular}{|c|c|c|c|c|c|}
\hline & $\begin{array}{l}\text { Strongly } \\
\text { disagree }\end{array}$ & Disagree & $\begin{array}{c}\text { Neither } \\
\text { agree } \\
\text { nor } \\
\text { disagree }\end{array}$ & Agree & $\begin{array}{c}\text { Strongly } \\
\text { agree }\end{array}$ \\
\hline I would like to play the game again. & 1 & 2 & 3 & 4 & 5 \\
\hline I would recommend the game to friends. & 1 & 2 & 3 & 4 & 5 \\
\hline I enjoyed playing the game. & 1 & 2 & 3 & 4 & 5 \\
\hline It was fun playing the game. & 1 & 2 & 3 & 4 & 5 \\
\hline I thought the game was too easy. & 1 & 2 & 3 & 4 & 5 \\
\hline This game is really boring. & 1 & 2 & 3 & 4 & 5 \\
\hline $\begin{array}{l}\text { I thought that game appealed to me } \\
\text { personally. }\end{array}$ & 1 & 2 & 3 & 4 & 5 \\
\hline $\begin{array}{l}\text { I could identify with the characters and the } \\
\text { stories. }\end{array}$ & 1 & 2 & 3 & 4 & 5 \\
\hline The game characters felt familiar. & 1 & 2 & 3 & 4 & 5 \\
\hline $\begin{array}{l}\text { I could empathize with the main character of } \\
\text { the game. }\end{array}$ & 1 & 2 & 3 & 4 & 5 \\
\hline $\begin{array}{l}\text { The game is useful for people trying to give } \\
\text { up smoking. }\end{array}$ & 1 & 2 & 3 & 4 & 5 \\
\hline The game was a waste of my time. & 1 & 2 & 3 & 4 & 5 \\
\hline $\begin{array}{l}\text { I learned a few things about the effects of } \\
\text { smoking. }\end{array}$ & 1 & 2 & 3 & 4 & 5 \\
\hline $\begin{array}{l}\text { People who want to give up smoking would } \\
\text { enjoy playing this game. }\end{array}$ & 1 & 2 & 3 & 4 & 5 \\
\hline This game would be useful for other people. & 1 & 2 & 3 & 4 & 5 \\
\hline
\end{tabular}


I got really involved in the game.

$\begin{array}{lllll}1 & 2 & 3 & 4 & 5 \\ 1 & 2 & 3 & 4 & 5\end{array}$

game.

I felt close to the main character.

$1+2 \quad 3$

4

If you have any comments on the game (this could be the content, the way it is structured and programmed), we would like to hear your opinion. Please use this space to list your comments and suggestions. 


\section{Bibliography}

[1] AAKER, J. Accessibility or diagnosticity? Disentangling the influence of culture on persuasion processes and attitudes. Journal of Consumer Research 26 (March 2000), 340 - 357.

[2] AAKER, J. Delineating culture. Journal of Consumer Psychology 16, 4 (2006), $343-347$.

[3] AakeR, J., Brumbaugh, A. M., AND Grier, S. M. Nontarget markets and viewer distinctiveness: The impact of target marketing on advertising attitudes. Journal of Consumer Research 9, 3 (2000), 127 - 140.

[4] AAKer, J., AND MAHesWARAN, D. The effect of cultural orientation on persuasion. The Journal of Consumer Research 24, 3 (December 1997), 315 328.

[5] AAKer, J., AND SChMitT, B. Culture-dependent assimilation and differentiation of the self: Preferences for consumption symbols in the United States and China. Journal of Cross-Cultural Psychology 32 (September 2001), $561-576$.

[6] AAKeR, J., AND Williams, P. Empathy versus pride: The influence of emotional appeals across cultures. The Journal of Consumer Research 25, 3 (December 1998), $241-261$.

[7] Airhinenbuwa, C. O., And KumanyiKa, S. Cultural aspects of African American eating patterns. Ethnicity \& Health 1, 3 (1996), 245 - 260.

[8] AjZen, I., And Madden, T. J. Predictions of goal-directed behaviour: Attitudes, intentions, and perceived behavioural controls. Journal of Experimental Social Psychology 22 (1986), 453 - 474.

[9] Alexander, C., Ishikawa, S., And Silverstein, M. A Pattern Language: Towns, Buildings, Construction. Center for Environmental Structure Series. Oxford University Press, August 1978. 
[10] AN, D. Reflection of cultural values in internet advertising in Korea and the U.S.: A theory-based content analysis. In Proceedings of the Association for Education in Journalism and Mass Communication Convention (2004).

[11] Apple INC. Nike + iPod. http://www . apple.com/ipod/nike/, October 2007. Last accessed 5 December 2007.

[12] Auahi Kore: Health Sponsorship Council. Auahi Kore. http:// www . auahikore.co.nz/, June 2005. Last accessed 5 December 2007.

[13] BADRE, A. N. The effects of cross cultural interface design orientation on world wide web user performance. Tech. Rep. GIT-GVU-01-03, Georgia Institute of Technology, College of Computing, GVU Center, 2003.

[14] Ball-Rokeach, S., Rokeach, M., AND Grube, J. Great American Values Test: Influencing Behavior and Belief through Television. Simon \& Schuster Adult Publishing Group, 1984.

[15] Bandura, A. Self-Efficacy: The Exercise of Control. W.H. Freeman and Company, New York, 1997.

[16] BARBER, W., AND BADRE, A. Culturability: The merging of culture and usability. In Proceedings of 4th Conference on Human Factors and the Web (1998).

[17] BarR, P., Biddle, R., AND Noble, J. Videogame values: Humancomputer interaction and games. Interacting With Computers 19, 2 (2006).

[18] BarR, P., Khaled, R., Noble, J., AND Biddle, R. Playing the interface: A case study of Grand Theft Auto: San Andreas. In OZCHI '06: Proceedings of the 20th conference of the computer-human interaction special interest group (CHISIG) of Australia on Computer-human interaction: design: activities, artefacts and environments (New York, NY, USA, 2006), ACM, pp. 317-320.

[19] BeLL, G. Designing for many futures. OzCHI 2006 keynote, November 2006.

[20] Berdichevsky, D., AND Neuenschwander, E. Toward an ethics of persuasive technology. Communications of the ACM 42, 5 (May 1999).

[21] Biasco, F., And Hartnett, J. P. Colleges students' attitudes toward smoking. In College Student Journal (September 2002).

[22] Biggs, J. B., AND Telfer, R. The Process of Learning. Prentice Hall, 1993. 
[23] Biswas, A., Olsen, J., AND CARlet, V. A comparison of print advertisements from the United States and France. Journal of Advertising 21, 4 (1992), $73-81$.

[24] Bogost, I. Persuasive Games: The Expressive Power of Videogames. The MIT Press, 2007.

[25] Bogost, I., AND FRASCA, G. Water Cooler Games. http://www . watercoolergames.org/, 2007. Last accessed 5 December 2007.

[26] BONANNI, C., AND CYR, D. Trust and loyalty: A cross-cultural comparison. In Proceedings for the International Conference of Business, Economics and Management Disciplines. (2004).

[27] Bordnick, P. S., GraAp, K. M., Copp, H., BroOKS, J., Ferrer, M., AND LOGUE, B. Utilizing virtual reality to standardize nicotine craving research: A pilot study. Addictive Behaviours 29 (2004), 1889 - 1894.

[28] Bramley, D., Riddell, T., Whittaker, R., Corbett, T., Lin, R.-B., Wills, M., JONES, M., AND RODGERS, A. Smoking cessation using mobile phone text messaging is as effective in Maori as non-Maori. The New Zealand Medical Journal 118, 1216 (June 2005).

[29] BRILEY, D., AND AAKER, J. When does culture matter? Effects of personal knowledge on the correction of culture-based judgement. Journal of Marketing Research 6, 3 (August 2006), 395-408.

[30] Burke, J. A., SalazaAr, A., Daughety, V., And Becker, S. L. Activating interpersonal influence in the prevention of adolescent tobacco use: An evaluation of Iowa's program against smoking. Health Communication 4, 1 (1992), 1 - 17.

[31] Centre For ExCELlence in Indigenous TOBACCO CONTROL. Why people smoke. http://www.ceitc.org.au/why_people_smoke/. Last accessed 5 December 2007.

[32] Chen, J., AND Ringel, M. Can advergaming be the future of interactive advertising?, 2001.

[33] Cialdini, R. B. Interpersonal influence. In Persuasion: Psychological Insights and Perspectives, T. C. Brock, Ed. Allyn and Beacon, 1994, pp. 195 217.

[34] Crawford, C. Chris Crawford on Game Design. New Riders Games, 2003. 
[35] CReswell, J. W. Research Design: Quantitative and Qualitative Approaches. Sage Publications, CA, 1994.

[36] CReswell, J. W. Qualitative Inquiry and Research Design: Choosing Amongst Five Traditions. Sage Publications, CA, 1998.

[37] DAUTENHAHN, K. Robots as social actors: AURORA and the case of autism. In Proceedings of the Third International Cognitive Technology Conference (CT99) (1999).

[38] DE MoOIJ, M. Global Marketing and Advertising: Understanding Cultural Paradoxes. Sage Publications, 2005.

[39] DijKstra, A., DE VRIES, H., KOK, G., AND RoijACKERS, J. Self-evaluation and motivation to change: Social cognitive constructs in quitting smoking. Psychology and Health 14 (1999), 747 - 759.

[40] Evers, V. Cultural Aspects of User Interface Understanding: An Empirical Evaluation of an E-Learning Website by International User Groups. PhD thesis, Institute of Educational Technology, The Open University, 2001.

[41] Eyck, A., Geerlings, K., Karimova, D., Meerbeek, B., WANG, L., IJsSelsteijn, W., De Kort, Y., Roersma, M., And Westerink, J. H. D. M. Effect of a virtual coach on athletes' motivation. In IJsselsteijn et al. [71], pp. 158-161.

[42] Festinger, L. A theory of social comparison. Human Relations 7 (1954), $117-140$.

[43] FogG, B. Persuasive computers: Perspectives and research directions. In CHI '98: Proceedings of the SIGCHI conference on Human factors in computing systems (1998), ACM Press / Addison-Wesley Publishing Co., pp. 225-232.

[44] FogG, B., Cutler, L. D., Arnold, P., And Eisbach, C. HandJive: A device for interpersonal haptic entertainment. In $\mathrm{CHI}$ '98: Proceedings of the SIGCHI conference on Human factors in computing systems (1998), ACM Press / Addison-Wesley Publishing Co., pp. 57-64.

[45] FogG, B., KamedA, T., Boyd, J., MARshall, J., Sethi, R., AND SOCKOL, M. Stanford-Makovsy web credibility study 2002: Investigating what makes web sites credible today. Report from the Persuasive Technology Lab, available online at http: / / captology . stanford.edu/pdf / Stanford-MakovskyWebCredStudy2002-pre\%lim.pdf, 2002. Last accessed 5 December 2007. 
[46] FogG, B. J. Persuasive Technology: Using Computers to Change What We Think and Do. Morgan Kaufmann Publishers, 2003.

[47] Fontaine, J. R., Poortinga, Y. H., Delbeke, L., And Schwartz, S. H. Structural equivalence of the values domain across cultures: Distinguishing sampling fluctuations from meaningful variation. In First Conference of the European Association of Survey Research (July 2005).

[48] Frasca, G., Battegazzore, S., Olhaberry, N., Infantozzi, P., RodrigueZ, F., AND BALBI, F. September the 12th. http://www. newsgaming.com/games/index12.htm, 2003. Last accessed 5 December 2007.

[49] Fullerton, T., Swain, C., And Hoffman, S. Game Design Workshop: Designing, Prototyping, and Playtesting Games. CMP Books, 2004.

[50] Gamma, E., Helm, R., JoHnson, R., AND Vlissides, J. Design patterns: Abstraction and reuse of object-oriented design. Lecture Notes in Computer Science 707 (1993), 406-431.

[51] GAO, G. Self and OTHER: A Chinese perspective on interpersonal relationships. In Communication in Personal Relationships Across Cultures, W. B. Gudykunst, S. Ting-Toomey, and T. Nishida, Eds. Sage Publications, CA, USA, 1996.

[52] GARITE, M. The ideology of interactivity (or video games and the Taylorization of leisure). In Level Up Conference Proceedings (November 2003), M. Copier and J. Raessens, Eds., University of Utrecht.

[53] GEE, J. P. What Video Games Have to Teach Us About Learning and Literacy. Palgrave Macmillan, 2003.

[54] GEORGE, S. E. Learning and the reflective journal in computer science. Australian Computer Science Communications 24, 1 (2002), 77-86.

[55] Gibson, J. J. The Ecological Approach to Visual Perception. Houghton Mifflin, Boston, MA, 1979.

[56] GoldER, S. A. The keep-in-touch phone: A persuasive telephone for maintaining relationships. In Extended abstracts of the 2004 conference on Human factors and computing systems (2004), ACM Press, pp. 1551-1551.

[57] Grimes, A., AND Grinter, R. E. Designing persuasion: Health technology for low-income African American communities. In Proceedings of The Second International Conference on Persuasive Technology (2007). 
[58] Grolleman, J., VAn DijK, B., Nijholt, A., AND van EMST, A. Break the habit! Designing an e-therapy intervention using a virtual coach in aid of smoking cessation. In IJsselsteijn et al. [71], pp. 133-141.

[59] Gudykunst, W. B., AND MAtsumoto, Y. Cross-cultural variability of communication in personal relationships. In Communication in Personal Relationships Across Cultures, W. B. Gudykunst, S. Ting-Toomey, and T. Nishida, Eds. Sage Publications, CA, USA, 1996.

[60] Gulliver, S. B., Hughes, J. R., Solomon, L. J., And Dey, A. N. An investigation of self-efficacy, partner support and daily stresses as predictors of relapse to smoking in self-quitters. Addiction 90, 6 (1995), 762 - 772.

[61] GÜRHAN-CANLI, Z., AND MAHESWARAN, D. Comparative advertising in the global marketplace: The effects of cultural orientation on communication. William Davidson Institute Working Papers Series 328, William Davidson Institute at the University of Michigan Business School, Aug. 2000. Available at http://ideas.repec.org/p/wdi/papers/ 2000-328.html. Last accessed 5 December 2007.

[62] HALL, E. Beyond Culture. Anchor Press/Doubleday, 1976.

[63] HAN, S.-P., AND SHAVITT, S. Persuasion and culture: Advertising appeals in individualistic and collectivistic societies. Journal of Experimental Social Psychology 30 (July 1994), 326 - 350.

[64] Hatvany, N., AND PUCIK, V. An integrated management system: Lessons from the Japanese experience. The Academy of Management Review 6, 3 (July 1981), $469-480$.

[65] Healthways QuitNet, INC. Quitnet. http: / / www . quitnet . com. Last accessed 5 December 2007.

[66] Heckman, C., ANd Wobbrock, J. Put your best face forward: Anthropomorphic agents, e-commerce consumers, and the law. In Proceedings of the ACM Conference on Autonomous Agents (AGENTS '00) (2000), pp. 435 - 442.

[67] HENRIKSEN, L., AND JACKSON, C. Anti-smoking socialization: Relationship to parent and child smoking status. Health Communication 10, 1 (1998), $87-101$.

[68] Hofstede, G. Culture's consequences. International differences in work-related values. Sage Publications, 1980. 
[69] Hofstede, G. Cultures and organisations: Software of the mind. McGraw-Hill Education, 1996.

[70] Holsti, O. R. Content Analysis for the Social Sciences and Humanities. Addison Wesley, 1969.

[71] IJsselsteijn, W., de Kort, Y., Midden, C. J. H., EgGen, B., AND VAN Den Hoven, E., Eds. Proceedings of the First International Conference on Persuasive Technology for Human Well-Being, PERSUASIVE 2006 (2006), vol. 3962 of Lecture Notes in Computer Science, Springer.

[72] Information SCIENCES Institute, University of SOUTHERn CALiFORNIA. Tactical Iraqi. Homepage at http: / / www . tacticallanguage . com/, 2003. Last accessed 5 December 2007.

[73] Institute FOR CReAtive TeChnologies, University OF SOUTHern CALIFORNIA. Virtual Iraq. Homepage at http://www.ict.usc.edu/ content/view/31/84/,2006. Last accessed 5 December 2007.

[74] Intille, S., KUKLA, C., FARZANFAR, R., AND BAKR, W. Just-in-time technology to encourage incremental, dietary behavior change. In Proceedings of the AMIA 2003 Symposium (2003), Wiley.

[75] IWAO, S. The Japanese Woman: Traditional Image and Changing Reality. Free Press, New York, 1993.

[76] Jenkins, H. Convergence Culture: Where Old and New Media Collide. NYU Press, 2006.

[77] Khaled, R., Barr, P., Fischer, R., Biddle, R., AND Noble, J. Factoring culture into the design of a persuasive game. In Proceedings of OzCHI 2006 (2006).

[78] Khaled, R., Barr, P., Noble, J., Fischer, R., ANd Biddle, R. Our place or mine? Exploration into collectivism-focused persuasive technology design. In IJsselsteijn et al. [71], pp. 72-83.

[79] Khaled, R., Biddle, R., Noble, J., Barr, P., And Fischer, R. Persuasive interaction for collectivist cultures. In The Proceedings of The Seventh Australasian User Interface Conference (2006), W. Piekarski, Ed.

[80] Khaled, R., Biddle, R., Noble, J., BARR, P., And Fischer, R. Persuasive interaction for collectivist cultures. In Proceedings of The Second International Conference on Persuasive Technology (2007). 
[81] Khaled, R., Noble, J., And Biddle, R. Developing culturally-aware persuasive technology. In Proceedings of the 7th International Workshop on Internationalisation of Products and Systems (2005), E. D. Gado, Ed.

[82] King, P., AND TeSter, J. The landscape of persuasive technologies. Communicaions of the ACM 42, 5 (May 1999).

[83] Kitayama, S., And Burnstein, E. Social influences, persuasion, and group decision making. In Persuasion: Psychological Insights and Perspectives, T. C. Brock, Ed. Allyn and Beacon, 1994, pp. 175 - 193.

[84] Klassen, R. M. Optimism and realism: A review of self-efficacy from a cross-cultural perspective. International Journal of Psychology 39, 3 (June 2004), $205-230$.

[85] Kleinjan, M., van den Eijnden, R. J. J. M., Dijkstra, A., Brug, J., AND ENGELS, R. C. M. E. Excuses to continue smoking: The role of disengagement beliefs in smoking cessation. Addictive Behaviours 31 (2006), 2223 $-2237$.

[86] Kobayashi, E., Grasmick, H., AND Friedrich, G. A cross-cultural study of shame, embarrassment, and managerial sanctions as deterrents to noncompliance with organizational rules. Communication Research Reports 18 (2001), $105-117$.

[87] Kotler, P., Roberto, N., AND Lee, N. Social Marketing: Improving the Quality of Life, 2 ed. Sage Publications, Thousand Oaks, 2002.

[88] Kroeber, A., AND Kluckhohn, C. Culture: A critical review of concepts and definitions. Harvard University Peabody Museum of American Archeology and Ethnology Papers 47, 1952.

[89] Langford, J., AND McDonagh, D., Eds. Focus Groups Supporting Effective Product Development. Taylor and Francis, 2002.

[90] LI, Z. The potential of America's Army the video game as civilian-military public sphere. Master's thesis, Massuchusetts Institute of Technology, 2003.

[91] LIN, C. Cultural differences in message strategies: A comparison between American and Japanese TV commercials. Journal of Advertising Research (July/August 1993), $40-48$.

[92] Macromedia, INC. Flash Professional 8 Education Version, 1993 - 2005. 
[93] MAHeSwARAN, D., AND SHAVITT, S. Issues and new directions in global consumer psychology. Journal of Consumer Psychology 9, 2 (2000), 59 - 66.

[94] Marcus, A., AND BAumgartner, V.-J. A practical set of culture dimensions for global user-interface development. In Computer-Human Interaction, 6th Asia Pacific Conference, APCHI 2004 (2004), M. Masoodian, S. Jones, and B. Rogers, Eds., pp. 252-261.

[95] Marcus, A., AND WEST, G. E. Crosscurrents: cultural dimensions and global web user-interface design. Interactions 7, 4 (2000), 32-46.

[96] MaRKUS, H., AND KitAyAmA, S. Culture and the self: Implications for cognition, emotion, and motivation. Psychological Review 98 (1991), 224 253.

[97] McCartney, E. S. Praise and dispraise in folklore. In The Evil Eye: A Casebook, A. Dundes, Ed. The University of Wisconsin Press, 1981.

[98] MCSWEENEY, B. Hofstede's model of national cultural differences and consequences: A triumph of faith - a failure of analysis. Human Relations 55, 1 (2002), $89-118$.

[99] Mestre, M., And Stainer, L. Employee orientation: The Japanese approach. Employee Relations 19 (November 1997), 443 - 456.

[100] Ministry of Social Development. The social report: Cigarette smoking. http://www.socialreport.msd.govt.nz/health/ cigarette-smoking.html, 2007. Last accessed 5 December 2007.

[101] National Institute of Health. Nicotine withdrawal. MedlinePlus Medical Encyclopedia (http://www.nlm.nih.gov/medlineplus/ encyclopedia.html, 2006. Last accessed 5 December 2007.

[102] Nelson, M. R., Keum, H., And Yaros, R. A. Advertainment or adcreep game players' attitudes toward advertising and product placements in computer games. Journal of Interactive Advertising 5, 1 (2004).

[103] Niaura, R., Abrams, D. B., Shadel, W. G., Rohsenow, D. J., Monti, P. M., AND SiROTA, A. D. Cue exposure treatment for smoking relapse prevention: A controlled clinical trial. Addiction 94, 5 (1999), 685 - 695.

[104] Nielsen, J., Ed. Designing User Interfaces for International Use. Elsevier Science Publishers, 1990. 
[105] Nielsen, J. Usability Engineering. Morgan Kaufmann Publishers Inc., San Francisco, CA, USA, 1995.

[106] Norman, D. A. The Psychology of Everyday Things. Basic Books, New York, 1990.

[107] O'Connell, K. A., Hosein, V. L., Schwartz, J. E., AND Leibowitz, R. Q. How does coping help people resist lapses during smoking cessation? Health Psychology 26, 1 (2007), 77 - 84.

[108] OISHI, S. Are ten value types necessary and sufficient? an examination of s. h. schwartz's (1994) theory of universal values. Master's thesis, University of Illinois, 1997.

[109] Patterson, J. Exploring Maori Values. Dunmore Press, 1992.

[110] Patterson, J. People of the Land: A Pacific Philosophy. Dunmore Press, 2000.

[111] PAVlou, P., AND CHAI, L. What drives electronic commerce across cultures? A cross-cultural empirical investigation of the theory of planned behaviour. Journal of Electronic Commerce Research 3, 4 (2002), 240 - 253.

[112] Pederson, P. Simulations: A safe place to take risks in discussing cultural differences. Simulation and Gaming 26, 2 (1995), 201-206.

[113] Petty, R., And CACiPpo, J. Communication and Persuasion: Central and Peripheral Routes to Attitude Change. Springer-Verlag, 1986.

[114] Petty, R. E., Cacioppo, J. T., Strathman, A. J., AND Priester, J. R. To think or not to think. In Persuasion: Psychological Insights and Perspectives, T. C. Brock, Ed. Allyn and Beacon, 1994, pp. 113 - 147.

[115] Prochaska, J., AND Diclemente, C. Stages and processes of self-change of smoking: Toward an integrative model of change. Journal of Consulting and Clinical Psychology 51 (1983), $390-395$.

[116] Quit Me Mutu Organisation. The Quit Group Website. http: //www . quit.org.nz/, September 2004. Last accessed 5 December 2007.

[117] Raybourn, E. M. Computer game design: New directions for intercultural simulation game designers. Developments in Business Simulation and Experiential Exercises 24 (1997).

[118] Redström, J. Persuasive design: Fringes and foundations. In IJsselsteijn et al. [71], pp. 112-122. 
[119] ReEves, B., AND NAss, C. The Media Equation: How People Treat Computers, Television, and New Media Like Real People and Places. University of Chicago Press, 1996.

[120] Rotzoll, K. Advertising in Contemporary Society: Perspectives Toward Understanding. Grid, Columbus, OH, 1976.

[121] Rouse, R. Game Design: Theory and Practice. Wordware Publishing, 2001.

[122] Ruiz, S., York, A., Stein, M., Keating, N., And Santiago, K. Darfur Is Dying. http://www.darfurisdying.com/, 2007. Last accessed 5 December 2007.

[123] Russo, P., AND BOOR, S. How fluent is your interface?: Designing for international users. In Proceedings of the SIGCHI conference on Human factors in computing systems (1993).

[124] SAWYER, B. Serious games: Improving public policy through game-based learning and simulation. Tech. Rep. 2002-1, Woodrow Wilson International Center for Scholars, Washington, D.C., 2002.

[125] SCHNEIDERMAN, B. Designing the User Interface. Addison-Wesley, 1992.

[126] Schoeffel, P., Meleisea, M., David, R., Kalauni, R., Kalolo, K., Kingitemaleti, P., Taumoefolau, Vuetibau, L., and Williams, S. P. Spare the rod? Conflicting cultural models of the family and approaches to child socialisation in New Zealand. Tech. Rep. 1, Centre of Pacific Studies, University of Auckland, 1994.

[127] SCHON, D. A. The Reflective Practitioner: How Professionals Think in Action. Basic Books, 1984.

[128] SCHWARTZ, S. Universals in the content and structure of values: Theoretical advances and empirical tests in 20 countries. In Advances in experimental social psychology, M. P. Zanna, Ed., vol. 25. New York: Academic Press, 1992, pp. $1-65$.

[129] SCHWARTZ, S. Mapping and interpreting cultural differences around the world. In Comparing Cultures, Dimensions of Culture in a Comparative Perspective, H. Vinken, J. Soeters, and P. Ester, Eds. Brill, 2003.

[130] SCHWARTZ, S., AND SAGIV, L. Identifying culture-specifics in the content and structure of values. Journal of Cross-Cultural Psychology 26, 1 (1995), 92 $-116$. 
[131] Sherman, C. Kicking butts. Psychology Today Magazine (September/October 1994).

[132] Sillence, E., Briggs, P., Fishwick, L., AND Harris, P. Trust and mistrust of online health sites. In CHI '04: Proceedings of the 2004 conference on Human factors in computing systems (2004), ACM Press, pp. 663-670.

[133] Silver, B. B. Social structure and games: A cross-cultural analysis of the structural correlates of game complexity. The Pacific Sociological Review 21, 1 (1978), 85 - 102.

[134] SINGH, S. Designing for money across borders. In Proceedings of HCI International (Las Vegas, Nevada, USA, 2005).

[135] Statistics New Zealand. 2001 Census. http://www. stats.govt nz /, 25 June 2002. Last accessed 5 December 2007.

[136] STUKE, F. Erfolgskontrolle von webespielen. Master's thesis, Ruhr Universität Bochum, 2002.

[137] TAFarodi, R. W., AND WALters, P. Individualism-collectivism, life events, and self-esteem: a test of two trade-offs. European Journal of Social Psychology 29, 5 (June 1999), 797 - 814.

[138] TAKemURA, K., AND YUKI, M. Are Japanese groups more competitive than Japanese individuals? A cross-cultural validation of the interindividual-intergroup discontinuity effect. International Journal of Psychology 42 (2007), $27-35$.

[139] TANSEY, R., HyMAN, M., AND ZinKHAN, G. Cultural themes in Brazilian and U.S. auto ads: A cross-cultural comparison. Journal of Advertising 19, 2 (1990), $30-39$.

[140] TelefoniCA. zZzZzZzZz. http://www.zzzzzzzzz.com.br, April 2006. Last accessed 7 April 2006, no longer available.

[141] Triandis, H. C. Culture and Social Behavior. McGraw-Hill Humanities/Social Sciences/Languages, 1994.

[142] TRIANDIS, H. C. Individualism and Collectivism (New Directions in Social Psychology). Westview Press, 1995.

[143] Triandis, H. C., Bontempo, R., Villareal, M. J., Asai, M., AND LUCCA, N. Individualism and collectivism: Cross-cultural perspectives 
on self-ingroup relationships. Journal of Personality and Social Psychology 54, 2 (1988), $323-338$.

[144] TRIANDIS, H. C., AND GELFAnd, M. J. Converging measurement of horizontal and vertical individualism and collectivism. Journal of Personality and Social Psychology 74, 1 (1998), 118 - 128.

[145] Turner, J. C. Social Influence. Brooks/Cole, Pacific Grove, CA, 1991.

[146] United States ARMY. America's army: The official army game. http: / / www . americasarmy . com/, 2002. Last accessed 5 December 2007.

[147] Vatrapu, R., And Perez-Quinones, M. A. Culture and international usability testing: The effects of culture in structured interviews. Journal of Usability Studies 1, 4 (August 2006), 156 - 170.

[148] VÖHRINGER-KUHNT, T. The influence of culture on usability. Master's thesis, Department of Educational Sciences and Psychology, Freie Universität Berlin, Berlin, Germany, 2001.

[149] Walton, M., AND VukOviC, V. HCI in the developing world: Cultures, literacy, and the web: dimensions of information "scent". Interactions 10, 2 (March 2003).

[150] Webster, A. Spiral of Values. Alpha Publications, 2001.

[151] Webster, S. Patrons of Maori Culture. University of Otago Press, 1998.

[152] Wells, W. D., Burnett, J., And Moriarty, S. Advertising - Principles and Practice. Eaglewood Cliffs, Prentice Hall, 1995.

[153] Wharton, C., Bradford, J., JefFries, R., And FranzKe, M. Applying cognitive walkthroughs to more complex user interfaces: Experiences, issues, and recommendations. In CHI '92: Proceedings of the SIGCHI conference on Human factors in computing systems (New York, NY, USA, 1992), ACM Press, pp. 381-388.

[154] Whatley, D. What's so serious about game design? The art or the science? Serious Games Summit DC 2005 keynote, 2005.

[155] WiEDERHOLD, D. B. K. Virtual reality games for smoking cessation. eHealth online 2, 11 (November 2007), 30 - 32. 
[156] Williams, J. H., Auslander, W. F., De Groot, M., Robinson, A. D., Houston, C., AND HAIRE-JOSHU, D. Cultural relevancy of a diabetes prevention nutrition program for African American women. Health Promotion Practice 7, 1 (2006), $56-67$.

[157] Williams, P., AND AAKER, J. Can mixed emotions peacefully co-exist? Journal of Consumer Research 4 (March 2002), 636 - 649.

[158] WinkLER, T., AND BUCKNER, K. Receptiveness of gamers to embedded brand messages in advergames: Attitudes towards product placement. Journal of Interactive Advertising 7, 1 (2006).

[159] YEO, A. W. Global-software development lifecycle: An exploratory study. In Proceedings of the SIGCHI conference on Human factors in computing systems (2001), ACM Press, pp. 104-111.

[160] Yi Hong, Y., Morris, M. W., yUe ChiU, C., AND Benet-Martinez, V. Multicultural minds: A dynamic constructivist approach to culture and cognition. American Psychologist 55, 7 (July 2000), 709 - 720.

[161] Yunus, M., AND JOLIS, A. Banker to the Poor: Micro-Lending and the Battle Against World Poverty. PublicAffairs, 1999.

[162] ZANDPOUR, F., AND SADRI, G. Communication in personal relationships in Iran: A comparative analysis. In Communication in Personal Relationships Across Cultures, W. B. Gudykunst, S. Ting-Toomey, and T. Nishida, Eds. Sage Publications, CA, USA, 1996.

[163] ZHANG, J., AND SHAVITT, S. Cultural values in advertisements to the Chinese X-generation. Journal of Advertising 32, 1 (2003), 21 - 31. 